Testes de Distância em Modelos de Regressão com Erros nas Variáveis

\author{
Celso Rômulo Barbosa Cabral
}

TESE APRESENTADA

AO

INSTITUTO DE MATEMÁTICA E ESTATÍSTICA

DA

UNIVERSIDADE DE SÃO PAULO

PARA

OBTENÇÃO DO GRAU DE DOUTOR

EM

ESTATÍSTICA

Área de concentração: Estatística

Orientador: Prof. Dr. Heleno Bolfarine

Co-orientador: Prof. Dr. Gilberto Alvarenga Paula

Durante a elaboração deste trabalho o autor recebeu apoio financeiro da CAPES

-São Paulo, março de 2000- 


\title{
TESTES DE DISTÂNCIA EM MODELOS DE REGRESSÃO COM ERROS NAS VARIÁVEIS
}

\author{
Este exemplar corresponde à redação \\ final da tese de doutoramento, \\ devidamente corrigida e defendida por \\ Celso Rômulo Barbosa Cabral e \\ aprovada pela comissão julgadora.
}

São Paulo, 17 de março de 2000.

Banca Examinadora:

Prof.Dr. Gilberto Alvarenga Paula - IME/USP

Prof.Dra. Sílvia Lopes de Paula Ferrari - IME/USP

Prof.Dr. Ruben Klein - LNCC

Prof.Dr. Enrico Colosimo - UFMG

Prof.Dr. Gauss Moutinho Cordeiro - UFBA 


\section{Dedicatória}

À minha família: Ivanete, Aluízio, Célio, Celly, Sylvana e Michelle. 


\section{Agradecimentos e Inspirações}

"Welcome back my friends to the show that never ends

We're so glad you could attend

Come inside! Come inside!

There behind a glass is a real blade of glass

be careful as you pass.

Move along! Move along!"

Karn Evil 9, de Keith Emerson e Greg Lake.

Meus sinceros agradecimentos vão para: Universidade do Amazonas e Universidade de São Paulo; Deborah Medeiros, Julio Hokama, Mário de Castro e Sebastião Lira (a turma de 96); Heleno Bolfarine e Gilberto Paula (orientadores); Flávia Morgana, Sílvia Fernandes (e sua maravilhosa família), José Cardoso Neto, Renato Tribuzy, Ruben Klein, Luís Gustavo Esteves (sofredor, graças a Deus!), Cláudia Daré, Gladys Salcedo, Daisy Tu, Jairo Eloy, Delhi Paiva, Regina Aquino e Odete Amaral (A penny for your thoughts my dear!). Que Deus abençoe a todos!

Inspirações e reflexões vieram de obras como Brain Salad Surgery (ELP), Foxtrot (Genesis) e Ok Computer (Radiohead), no lado musical, Office Space (de Mike Judge) e Happiness (de Todd Solondz), na parte cinematográfica e O Último Teorema de Fermat (de Simon Singh), na área da literatura; no setor esportivo, as brilhantes vitórias do esquadrão de São Januário ("Tua estrela, na terra a brilhar, ilumina o mar"). Estas obras-primas deram um pouco de alívio às tensões em quatro anos de doutoramento. 
Nouvelles tendances, nouvelles idées, nouvelle vie Slogan feito para os Mutantes, em Paris. 


\section{Resumo}

Apresentamos uma aplicação da teoria geral dos testes de distância a modelos com erros nas variáveis. Consideramos o modelo de regressão linear simples com intercepto nulo, o modelo de regressão multivariado e um caso especial deste, o modelo de calibração comparativa. Comparamos, utilizando dados simulados, a função poder do teste de distância para hipóteses com restrições nos parâmetros com as funções poder de outros testes gerais, formulados sem incorporar em suas estruturas as suposições de erros nas variáveis e hipóteses restritas simultaneamente. Sabemos que, quando a matriz jacobiana da função que descreve as restrições nas hipóteses tem posto completo, a distribuição nula assintótica da estatística do teste de distância é uma mistura de distribuições qui-quadrado. Isto não é necessariamente verdadeiro quando existem pontos singulares na hipótese nula. Sugerimos uma modificação na estatística de teste que assegura a convergência à uma mistura de distribuições quiquadrado. Dois conjuntos de dados reais são analisados de acordo com as técnicas propostas. 


\begin{abstract}
We present an application of the general theory of distance tests to errors-invariables models. We consider the simple linear regression model with null intercept, the multivariate regression model and a special case of that, the comparative calibration model. We compare, using artificial data, the power function of the distance test for restricted hypotheses with power functions of another general tests that were developed without the assumptions of errors in variables and restricted hypotheses occurring simultaneously. It is known that, when the jacobian matrix of the restriction function describing the hypotheses has full rank, the asymptotic null distribution of the distance test statistic is a mixture of chi-square distributions. This assertion is not necessarily true when there exist singular points in the null hypothesis. We suggest a modification to the test statistic which ensures convergence to a mixture of chi-square distributions. Two sets of real data are analysed according to the proposed methods.
\end{abstract}




\section{Conteúdo}

Algumas Notações, Definições e Resultados Básicos 3

1 Introdução $\quad 7$

2 O Teste de Distância 11

2.1 Conceitos Básicos . . . . . . . . . . . . . . . . 11

2.2 O Teste de Distância para Restrições de Igualdade e Desigualdade . . 16

3 Testes para Hipóteses Restritas em Modelos de Regressão Linear Simples com Erros nas Variáveis $\quad 28$

3.1 Alguns Modelos de Regressão com Erros nas Variáveis . . . . . . . . 28

3.1 .1 Modelo Geral . . . . . . . . . . . . . . . . . . . . 28

3.1.2 Modelo Passando Pela Origem . . . . . . . . . . . . . . 29

3.1.3 Modelo com a Razão das Variâncias dos Erros de Observação

Conhecida . . . . . . . . . . . . . 33

3.2 Testes de Distância para Algumas Hipóteses Restritas . . . . . . . . . 34

3.2.1 Modelo Passando Pela Origem . . . . . . . . . . . . . . 34

3.2 .2 Uma Aplicação . . . . . . . . . . . . . . . . . . . . . . . . . . . . . . . . . 38

3.2.3 Modelo com Razão de Variâncias Conhecida . . . . . . . . . . 39

3.3 Extensões para Duas ou Mais Retas . . . . . . . . . . . . . . . 40

4 O Modelo de Regressão Linear Múltiplo Multivariado $\mathbf{5 1}$

4.1 Definição do Modelo e Estimação de Máxima Verossimilhança . . . . 51

4.2 Um Teste para a Qualidade do Ajuste . . . . . . . . . . . . . . . . . 62

4.3 Consistência . . . . . . . . . . . . . . . . 65

4.4 Normalidade Assintótica . . . . . . . . . . . . . . . . . . . . 69

4.5 Testes de Distância para Algumas Hipóteses Restritas . . . . . . . . 78 
5 Testes de Distância sob Condições Não-regulares: Aplicações ao $\begin{array}{lr}\text { Modelo de Calibração Comparativa } & 87\end{array}$

5.1 Definição do Modelo . . . . . . . . . . . . . . . . . 87

5.2 Estimação de Máxima Verossimilhança no MRMC . . . . . . . . . . . 89

5.3 Um Teste para a Qualidade do Ajuste . . . . . . . . . . . . . . . . . . . . . . . . . . . . . . . . . . . . .

5.4 Consistência . . . . . . . . . . . . . . . . . . . 92

5.5 Normalidade Assintótica . . . . . . . . . . . . . . . . . 93

5.6 Dificuldades de Aplicação dos Testes de Wald e de Distância e um Teste de Distância Modificado . . . . . . . . . . . . . . . . . . . . . . 98

5.7 Uma Aplicação . . . . . . . . . . . . . . . . . . . . . . . . 104

Conclusões, Contribuições e Propostas para Futuras Pesquisas 113

A Procedimento Computacional para o Cálculo da Estatística de Distância

B Expressões para os Pesos na Distribuição Nula Assintótica da Estatística de Distância 


\section{Algumas Notações, Definições e Resultados Básicos}

Apresentamos aqui notações, definições e resultados simples, mas extremamente úteis, que aparecem com grande freqüencia na tese.

\section{Vetores e Matrizes}

$A^{\prime}$ denota a transposta da matriz $A$. Um mesmo símbolo pode ser utilizado para denotar objetos ou relações diferentes, mas as coisas ficarão claras no contexto em que forem utilizadas. Assim, $|A|$ representa o determinante da matriz $A$ e $|x|=\max \{x,-x\}$ representa módulo do número real $x$. 0 é usado indistintamente para denotar matrizes nulas de qualquer ordem. $I_{p}$ é a matriz identidade de ordem $p \times p$ (algumas vezes eliminaremos o subscrito $p$ ). Uma notação semelhante é utilizada para a função indicadora de um conjunto $C$, definida por $I_{C}(x)=1$ se $x \in C$ e $I_{C}(x)=0$ se $x \notin C$. Se $A_{i j}$ é o elemento de ordem $(i, j)$ da matriz $A$ escrevemos $A=\left(A_{i j}\right)$. Esta notação também é utilizada para representar uma partição de $A$ em blocos $A_{i j}$. Caso $A$ seja uma matriz diagonal (diagonal em blocos) escrevemos $A=\operatorname{diag}\{\cdot\}(A=$ block $\operatorname{diag}\{\cdot\})$, especificando entre chaves os elementos (blocos) da diagonal. Se $A$ é simétrica, $A>0(A \geq 0)$ significa que $A$ é positiva definida (não-negativa definida). $\mathcal{M}_{p}$ denota o conjunto formado pelas matrizes simétricas $A: p \times p>0$. Um vetor $x \in \mathbb{R}^{p}$ é representado na forma de matriz coluna: escrevemos este vetor em termos de suas coordenadas na base canônica de $\mathbb{R}^{p}$ como $x=\left(x_{1}, \ldots, x_{p}\right)^{\prime}$. Se $c=\left(c_{1}, \ldots, c_{p}\right)^{\prime}$, então $x \geq c$ significa que $x_{i} \geq c_{i}, i=1, \ldots, p$. Analogamente para $x \leq c, x>c, x<c$. Seja $A \in \mathcal{M}_{p}$. A função $<x, y>_{A}=x^{\prime} A^{-1} y$, $(x, y) \in \mathbb{R}^{p} \times \mathbb{R}^{p}$, define um produto interno em $\mathbb{R}^{p}$. A norma proveniente deste produto, denominada norma em $\mathbb{R}^{p}$ induzida por $A$, é definida por $\|x\|_{A}^{2}=x^{\prime} A^{-1} x$. 
Se $A=I$ escrevemos simplesmente $\|x\|$. $\mathcal{O}_{p}$ é o conjunto $\left\{x \in \mathbb{R}^{p} ; x \geq 0\right\}$.

\section{Funções}

Sejam $E, F$ e $G$ conjuntos, $g: E \times F \rightarrow G$ uma aplicação e $(a, b) \in E \times F$. Definimos as aplicações parciais $g_{a}: F \rightarrow G$ e $g_{b}: E \rightarrow G$ por $g_{a}(x)=g(a, x)$ e $g_{b}(x)=g(x, b)$. Se $E \subset \mathbb{R}^{p}$ é aberto e $G=\mathbb{R}^{q}$ definimos a primeira derivada parcial de $g$ no ponto $(a, b), \partial_{1} g(a, b): \mathbb{R}^{p} \rightarrow \mathbb{R}^{q}$, como sendo a derivada de $g_{b}$ no ponto $a$. Analogamente, definimos a segunda derivada parcial. Seja $f: A \rightarrow \mathbb{R}^{q}$ uma aplicação definida no aberto $A \subset \mathbb{R}^{p}$. Dizemos que $f$ é de classe $C^{k}$ quando a $k$-ésima derivada $f^{(k)}(x)$ existe em todo ponto $x \in A$ e a aplicação $x \mapsto f^{(k)}(x)$, $x \in A$ é contínua. A restrição de uma função $g: E \rightarrow F$ a um conjunto $D \subset E$ é denotada por $\left.g\right|_{D}: D \rightarrow F$. Assim, $\left.g\right|_{D}(x)=g(x), x \in D$.

\section{Probabilidade e Estatística}

Quando o vetor aleatório $p$-dimensional $X$ tiver distribuição normal com vetor de médias $\mu$ e matriz de covariâncias $\Sigma$, escreveremos $X \sim N_{p}(\mu, \Sigma)$, ou simplesmente $X \sim N(\mu, \Sigma) \cdot \chi_{p}^{2}$ denota uma variável aleatória com distribuição qui-quadrado com $p$ graus de liberdade.

Se $F_{i}, i=1, \ldots, p$, são funções de distribuição então $F=\sum_{i=1}^{p} \omega_{i} F_{i}, \omega_{i} \geq 0, i=$ $1, \ldots, p, \sum_{i=1}^{p} \omega_{i}=1$, também é uma função de distribuição, denominada mistura de $F_{i}, i=1, \ldots, p$. $\omega_{i}$ são os pesos da mistura.

Como trabalhamos com teoria assintótica, é necessário definir espaços de probabilidade para amostras infinitas. Assim, seja $\left(\Phi, \mathcal{A}, \mu_{\theta}\right)$ um espaço de probabilidade, onde $\theta \in \Theta \subset \mathbb{R}^{k}$ é um parâmetro desconhecido. Considere o espaço mensurável formado pelo conjunto

$$
\Psi=\prod_{i=1}^{\infty} \mathbb{R}^{p}=\left\{\left(x_{1}, x_{2}, \ldots\right) ; x_{i} \in \mathbb{R}^{p}, i=1,2, \ldots\right\}
$$

e pela $\sigma$-álgebra produto $\mathcal{F}=\prod_{i=1}^{\infty} \mathcal{B}\left(\mathbb{R}^{p}\right)$, onde $\mathcal{B}\left(\mathbb{R}^{p}\right)$ é a $\sigma$-álgebra de Borel em $\mathbb{R}^{p}$. Definimos uma amostra aleatória proveniente de uma população com distribuição $Q_{\theta}$ 
como sendo uma função mensurável ${ }^{1}$

$$
X=\left(X_{1}, X_{2}, \ldots\right):(\Phi, \mathcal{A}) \rightarrow(\Psi, \mathcal{F})
$$

tal que $X_{i}, i=1,2, \ldots$ são independentes e possuem a mesma distribuição de probabilidade

$$
Q_{\theta}(A)=\mu_{\theta}\left(X_{1} \in A\right), A \in \mathcal{B}\left(\mathbb{R}^{p}\right) .
$$

Definimos a distribuição da amostra aleatória $X$ por

$$
P_{\theta}(A)=\left(\prod_{i=1}^{\infty} Q_{\theta}\right)(A), A \in \mathcal{F},
$$

onde $\prod_{i=1}^{\infty} Q_{\theta}$ é a medida produto. $P_{\theta}$ é uma medida de probabilidade definida em $\mathcal{F}$, para todo $\theta \in \Theta$. Entendemos por modelo estatístico a coleção de espaços de probabilidade $\left\{\left(\Psi, \mathcal{F}, P_{\theta}\right) ; \theta \in \Theta\right\}$. Qualquer função mensurável definida em um destes espaços de probabilidade e com imagem no espaço Euclideano é denominada estatística. Se uma estatística é usada com o objetivo de estimar $\theta$ a chamamos de estimador de $\theta$. $\Theta$ é o espaço paramétrico associado ao modelo.

Fixando $\left(\Psi, \mathcal{F}, P_{\theta}\right)$, convergências em probabilidade, quase certa e em distribuição são denotadas por $\stackrel{\operatorname{Pr}(\theta)}{\longrightarrow}, \stackrel{\mathrm{qc}(\theta)}{\longrightarrow} \mathrm{e} \stackrel{\mathcal{D}(\theta)}{\longrightarrow}$, respectivamente. Assim, uma sequência $\left\{\hat{\theta}_{n}\right\}$ de estimadores $^{2}$ de $\theta$ é fracamente consistente quando $\hat{\theta}_{n} \stackrel{\operatorname{Pr}(\theta)}{\longrightarrow} \theta$ para todo $\theta \in \Theta$, e é fortemente consistente quando $\hat{\theta}_{n} \stackrel{\mathrm{qc}(\theta)}{\longrightarrow} \theta$ para todo $\theta \in \Theta$.

$\mathrm{O}$ modelo $\left\{\left(\Psi, \mathcal{F}, P_{\theta}\right) ; \theta \in \Theta\right\}$ é dito ser identificável quando para todos $\theta_{1}, \theta_{2} \mathrm{em}$ $\Theta$ tais que $P_{\theta_{1}}=P_{\theta_{2}}$ tivermos $\theta_{1}=\theta_{2}$. Pela unicidade dos limites em probabilidade e quase certo, temos que a existência de sequências de estimadores de $\theta$ consistentes só é possível quando o modelo é identificável.

Uma reparametrização do modelo estatístico $\left\{\left(\Psi, \mathcal{F}, P_{\theta}\right) ; \theta \in \Theta\right\}$ é uma bijeção $f$ entre um subconjunto do espaço Euclideano $\Xi$ e $\Theta$. O modelo estatístico reparametrizado é dado por $\left\{\left(\Psi, \mathcal{F}, Q_{\xi}\right) ; \xi \in \Xi\right\}$, onde $Q_{\xi}=P_{\theta}, f(\xi)=\theta$. Algumas propriedades são preservadas por reparametrizações. Se o modelo original é identificável, o modelo reparametrizado também o é; se $T\left(X_{1}, \ldots, X_{n}\right)$ é um estimador de máxima verossimilhança para $\theta$ baseado em $n$ observações, então $f^{-1}\left[T\left(X_{1}, \ldots, X_{n}\right)\right]$ é estimador de máxima verossimilhança para $\xi$, onde $f^{-1}$ é a inversa de $f$; se $f^{-1}$

\footnotetext{
${ }^{1}$ Observe que $X$ é mensurável se e somente se cada função coordenada $X_{i}:(\Phi, \mathcal{A}) \rightarrow\left(\mathbb{R}^{p}, \mathcal{B}\left(\mathbb{R}^{p}\right)\right)$ é mensurável (Ash, 1972, teorema 5.11.3).

${ }^{2}$ em geral, temos $\hat{\theta}_{n}\left(x_{1}, x_{2}, \ldots\right)=T_{n}\left(x_{1}, \ldots, x_{n}\right)$, onde $T_{n}: \prod_{i=1}^{n} \mathbb{R}^{p} \rightarrow \mathbb{R}^{k}$ é Borel mensurável.
} 
é contínua e $\left\{\hat{\theta}_{n}\right\}$ é uma sequência de estimadores consistente no modelo original, então $\left\{f^{-1}\left(\hat{\theta}_{n}\right)\right\}$ também é consistente no modelo reparametrizado. Se $f^{-1}$ é diferenciável e $\left\{n^{1 / 2}\left(\hat{\theta}_{n}-\theta\right)\right\}$ tem distribuição limite normal, então $\left\{n^{1 / 2}\left[f^{-1}\left(\hat{\theta}_{n}\right)-f^{-1}(\theta)\right]\right\}$ também tem distribuição limite normal.

Sejam $X$ e $Y$ amostras aleatórias. A não ser quando explicitamente mencionado em contrário, usamos a notação $S_{X Y}$ para representar a sequência de covariâncias amostrais entre $X$ e $Y$, ou seja,

$$
S_{X Y}=\frac{1}{n} \sum_{i=1}^{n}\left(X_{i}-\bar{X}\right)\left(Y_{i}-\bar{Y}\right)^{\prime},
$$

$\bar{X}=n^{-1} \sum_{i=1}^{n} X_{i}, \bar{Y}=n^{-1} \sum_{i=1}^{n} Y_{i}$. Se $X=Y$, escrevemos $S_{X}$. Um resultado bastante conhecido é que, se $X=\left(X_{1}, \ldots, X_{n}\right)$ é uma amostra aleatória (finita) proveniente de uma distribuição normal de qualquer dimensão, então $\bar{X}$ e $S_{X}$ são os estimadores de máxima verossimilhança para a média e a matriz de covariâncias, respectivamente.

\section{Simbolos Especiais}

O símbolo $\square$ indica o fim de uma demonstração. 


\section{Capítulo 1}

\section{Introdução}

Nesta tese trabalhamos com modelos de regressão com erros nas variáveis. Isto significa que, diferentemente da situação clássica, os regressores não são observados diretamente, e o que realmente observamos são os seus verdadeiros valores mais um erro aleatório ${ }^{1}$. Se os verdadeiros valores são constantes desconhecidas usamos a denominação modelo funcional; se são variáveis ou vetores aleatórios, modelo estrutural $^{2}$. Somente o modelo estrutural será considerado. Estudamos modelos de regressão linear simples (com intercepto nulo e com intercepto arbitrário) e regressão linear múltipla (univariada e multivariada).

Um primeiro contato com modelos de regressão (com ou sem erros nas variáveis) geralmente é feito com modelos irrestritos, no sentido de que o espaço paramétrico associado é o mais abrangente possível: parâmetros de inclinação e intercepto variando livremente no espaço Euclideano, variâncias dos erros de observação positivas e, no caso de erros nos regressores, variância dos regressores positiva. Estimação e testes nesta situação são abordados em textos como Seber (1977) e Jørgensen (1993), para os modelos clássicos, Fuller (1987) e Cheng \& Van Ness (1999) para os modelos com erros nas variáveis. Ocorre que em certos experimentos a natureza do problema nos permite supor que alguns parâmetros variam de uma forma mais restrita. Dois exemplos do mundo real serão utilizados na tese. O primeiro será analisado no capítulo 3: em um curso de línguas, estudantes são submetidos a testes auditivos

\footnotetext{
${ }^{1}$ Neste caso, dizemos que o modelo tem erros aditivos. Existem outras formas de incorporar os erros ao modelo como, por exemplo, considerá-los multiplicativos, mas isto não será objeto de nosso estudo.

${ }^{2}$ As duas situações são casos particulares do modelo ultraestrutural, apresentado por Dolby (1976).
} 
antes (pré-teste) e após (pós-teste) o curso. Este experimento pode ser modelado utilizando-se modelos de regressão linear simples com intercepto nulo. As variáveis relacionadas seriam os níveis de conhecimento do estudante no pré-teste e no pósteste. É absolutamente razoável supor que o nível de conhecimento do estudante ao final do curso seja maior ou igual que o nível de conhecimento no início do curso. Portanto é realista a suposição de que o parâmetro de inclinação $\beta$ é maior ou igual a 1. Então, é importante testar $\beta=1$ (o nível de conhecimento permanece o mesmo) contra $\beta>1$ (uma melhora no nível de conhecimento).

Um outro exemplo é o problema de calibração linear comparativa, analisado no capítulo 5. Trata-se da comparação de instrumentos de medição segundo critérios como precisão e não viciosidade, que serão bem definidos no citado capítulo. $\mathrm{O}$ conjunto de dados apresentado refere-se a medições de volume testicular em adolescentes, usando-se três métodos. Um deles é o Ultrasom, os outros são métodos que se baseiam em inspeção visual. Qualquer que seja a definição de precisão, é natural supor que o método Ultrasom é mais ou tão preciso quanto os demais. Seria interessante testar a hipótese que os métodos são igualmente precisos contra uma hipótese de ordenação das precisões.

Seja $\theta$ o vetor de parâmetros do modelo. Entendemos por hipóteses restritas aquelas que podem ser colocadas na forma $H: h(\theta)=\left(h_{1}(\theta), h_{2}(\theta)\right)=0$ (hipótese nula) e $K: h_{2}(\theta) \geq 0$ (hipótese alternativa), onde $h$ é uma função vetorial conveniente. Estudamos nesta tese a performance de diversos testes para $H$ contra $K$. Um dos testes, o teste de distância, é elaborado incorporando em sua definição a natureza restrita de $K$ e a suposição de erros nas variáveis. Os outros não satisfazem uma ou outra destas condições. Isto faz com que, invariavelmente, tenham um desempenho em termos de poder inferior ao do teste de distância.

Os objetivos gerais do trabalho são os seguintes: (i) elaborar testes de distância para hipóteses restritas em modelos de regressão com erros nas variáveis e (ii) comparar a função poder do teste de distância com funções poder de outros testes que são formulados sem incorporar conjuntamente em suas definições as suposições de erros nas variáveis e hipóteses restritas.

$\mathrm{Na}$ discussão que segue consideramos $\mathbb{R}^{p}$ munido da distância definida pela norma induzida por $\Sigma$. A definição do teste de distância é inspirada no seguinte problema: seja $X \sim N_{p}(\mu, \Sigma)$, onde $\Sigma>0$ é conhecida. Seja $C$ um cone ${ }^{3}$. A estatística do teste

\footnotetext{
${ }^{3}$ Para efeito desta introdução, $C$ é convexo e $\alpha x \in C$ para todos $\alpha>0$ e $x \in C$. No capítulo 2 usaremos uma definição ligeiramente diferente.
} 
da razão de verossimilhança para

$$
\mu=0 \text { contra } \mu \in C
$$

é dada por

$$
R V=X^{\prime} \Sigma^{-1} X-\min \left\{(X-z)^{\prime} \Sigma^{-1}(X-z) ; z \in C\right\}
$$

e rejeitamos $\mu=0$ para grandes valores de $R V$. Se a hipótese $\mu=0$ é verdadeira, a distribuição de $R V$ é uma mistura de funções de distribuição qui-quadrado, um resultado demonstrado por Shapiro (1985), generalizando resultados de Kudô (1963) e Nüesch $(1966)$ - estes últimos tratam o caso $C=\mathcal{O}_{p}$. Os pesos $\omega_{i}$ da mistura dependem de $\Sigma$ e $C$. Se $C=\mathcal{O}_{p}$ então $\omega_{i}$ é igual a probabilidade de que a projeção de $X \sim N(0, \Sigma)$ sobre $C$ tenha exatamente $i$ coordenadas positivas.

Para implementarmos o teste da razão de verossimilhança precisamos, obviamente, do valor dos pesos. Mas, infelizmente, formas analíticas fechadas só existem para $p \leq 4$. Estas expressões estão no Apêndice B. Procedimentos computacionais para o cálculo dos pesos podem ser encontrados em Bohrer \& Chow (1978) ou Sun (1988a,b).

Se $C=\mathbb{R}^{p}$ então $R V=X^{\prime} \Sigma^{-1} X$, que tem distribuição qui-quadrado quando $X \sim$ $N(\mu, \Sigma)$ (a distribuição é não central quando $\mu \neq 0$ ). Não é possível estender este resultado para outras formas de $C$, ou seja, sob a hipótese alternativa a distribuição de $R V$ não é necessariamente uma mistura de funções de distribuição qui-quadrado não centrais (Shapiro, 1988, §8). Em geral, e esta tese não é uma exceção, os estudos de poder para o teste de distância têm sido feito através de dados gerados artificialmente.

$R V$ é uma diferença das distâncias ao quadrado de $X$ a dois conjuntos: $\{0\}$ e $C$. Isto é uma motivação para a definição do teste de distância para $H$ contra $K$, dada por Kodde \& Palm (1986). A idéia: freqüentemente a sequência de estimadores de máxima verossimilhança para $\theta,\left\{\hat{\theta}_{n}\right\}$, é consistente e tem distribuição assintótica $N\left(n^{-1 / 2} \theta, n^{-1} \Sigma\right), \Sigma=\Sigma(\theta)$. Conseqüentemente, $h\left(\hat{\theta}_{n}\right)$ tem distribuição assintótica normal com média $n^{-1 / 2} h(\theta)$ (que sob $H$ é igual a zero) e uma matriz de covariâncias que depende de $\theta$, digamos, $n^{-1} \Omega(\theta)$. Definimos então a estatística de distância (D) da mesma forma que definimos $R V$, com $h(\hat{\theta})$ no lugar de $X$ e $n^{-1} \Omega(\hat{\theta})$ no lugar de $\Sigma$. $\mathrm{O}$ teste de distância rejeita $H$ para grandes valores de $\mathbf{D}$. No capítulo 2 provamos que a distribuição nula limite de $\mathbf{D}$ é exatamente igual à distribuição de $R V$, quando $C=\mathbb{R}^{q} \times \mathcal{O}_{p-q}$ e $X \sim N(0, \Omega(\theta))$. A demonstração usa argumentos de Perlman 
$(1969)^{4}$.

Uma dificuldade para a implementação do teste é a dependência dos pesos em relação a $\theta$. Isto faz com que a distribuição nula limite de $\mathbf{D}$ também dependa de $\theta$. Neste caso, encontrar o valor crítico $c_{\alpha}$ para um teste com nível de significância $\alpha$, solução de

$$
\sup \left\{\lim _{n \rightarrow \infty} P_{\theta}\left(\mathbf{D} \geq c_{\alpha}\right) ; h(\theta)=0\right\}=\alpha
$$

pode ser uma tarefa árdua. No capítulo 2 apresentamos uma sugestão para contornar o problema.

Os capítulos restantes tratam da aplicação da teoria desenvolvida no capítulo 2 aos diversos modelos com erros nas variáveis já citados. Para estes modelos, exibimos estimadores de máxima verossimilhança e provamos que estes são consistentes e assintoticamente normais. Esta exposição é baseada nos artigos de Chan \& Mak (1979) (capítulo 3) e Amemiya \& Fuller (1984) (capítulo 4). As simulações por computador apresentam evidências de que o teste de distância é mais poderoso que outros testes que não estão de acordo com as suposições feitas para o modelo.

O teste de distância na forma como foi definido anteriormente não é aplicável quando desejamos testar algumas hipóteses de interesse no modelo de calibração linear comparativa. A função $h$ utilizada para parametrizar $H$ e $K$ possui derivada igual a zero em alguns pontos da hipótese nula e, como veremos no capítulo 5 , tal fato prejudica a convergência em distribuição de $\mathbf{D}$ a uma mistura de distribuições quiquadrado, fazendo com que o teste seja extremamente conservador. Apresentamos então uma modificação do teste de distância que, aparentemente, resolve o problema de maneira satisfatória.

\footnotetext{
${ }^{4}$ Este trabalho trata do teste da razão de verossimilhança para (1.1) quando $\Sigma$ é desconhecida.
} 


\section{Capítulo 2}

\section{O Teste de Distância}

Neste capítulo apresentamos o teste de distância para hipóteses que podem ser colocadas na forma $h(\theta)=\left(h_{1}(\theta), h_{2}(\theta)\right)=0$ (hipótese nula) e $h_{2}(\theta) \geq 0$ (hipótese alternativa), onde $h$ é uma função conveniente e $\theta$ é o parâmetro associado a um determinado modelo. Alguns resultados de análise convexa, necessários para a elaboração da teoria, são enunciados sem demonstração. Obtemos a distribuição nula assintótica da estatística de teste e observamos que uma dificuldade para a implementação do teste é que esta distribuição depende de $\theta$. Sugerimos então um procedimento para contornar este problema.

\subsection{Conceitos Básicos}

Seja $H \subset \mathbb{R}^{p}$ um subespaço vetorial de dimensão $p-1$ (um hiperplano). Como o complementar ortogonal de $H$ tem dimensão 1 , existe $a \in \mathbb{R}^{p}$ não nulo tal que $H=\left\{x \in \mathbb{R}^{p} ; a^{\prime} x=0\right\}$ e $H=H^{+} \cap H^{-}$, onde $H^{+}=\left\{x \in \mathbb{R}^{p} ; a^{\prime} x \geq 0\right\}$ e $H^{-}=\left\{x \in \mathbb{R}^{p} ; a^{\prime} x \leq 0\right\}$ são denominados semi-espaços. Dizemos que $D \subset \mathbb{R}^{p}$ não vazio é positivamente homogêneo se $x \in D$ implicar em $\alpha x \in D$ para todo $\alpha>0$. Um conjunto $A \subset \mathbb{R}^{p}$ que contém pelo menos um elemento diferente de zero é dito ser unilateral se existir $z \in \mathbb{R}^{p}$ não nulo tal que $x^{\prime} z>0$ para todo $x \neq 0, x \in A$. Se $D \subset \mathbb{R}^{p}$ é positivamente homogêneo, fechado e unilateral dizemos então que $D$ é um cone. $\mathcal{O}_{p}=\left\{x \in \mathbb{R}^{p} ; x \geq 0\right\}$ é um exemplo de cone, assim como uma semi-reta $L=\{\alpha x ; \alpha \geq 0\}$, onde $x \in \mathbb{R}^{p}$ é fixo, e um cone circular à direita, definido da 
seguinte maneira:

$$
C_{\lambda}=\left\{x \in \mathbb{R}^{p} ; \frac{x^{\prime} z}{\left[\left(x^{\prime} x\right)\left(z^{\prime} z\right)\right]^{1 / 2}} \geq \lambda\right\}
$$

onde $\lambda \in(0,1]$ e $z \in \mathbb{R}^{p}-\{0\}$ são fixos.

Os resultados a seguir formam a base para a formulação do teste de distância, apresentada na seção 2.2. Com exceção do lema 2.1.1 e do teorema 2.1.4, todos foram extraídos de Perlman (1969). $T(C)$ denota a imagem do conjunto $C$ pela transformação $T$.

Teorema 2.1.1 Sejam $C$ um cone e $C_{\lambda}, \lambda \in(0,1)$, um cone circular à direita. Então

1. Existe um cone circular à direita, $C_{\phi}, \phi \in(0,1)$ tal que $C \subset C_{\phi}$;

2. Se $C$ tem interior não vazio, existe um cone circular à direita, $C_{\nu}, \nu \in(0,1)$ tal que $C_{\nu} \subset C$;

3. Existe uma semi-reta $L$ e uma sequência de transformações lineares invertíveis $\left\{A_{n}, n \in \mathbb{N}\right\}$, tais que

$$
A_{n}\left(C_{\lambda}\right) \supset A_{n+1}\left(C_{\lambda}\right), \quad \bigcap_{n=1}^{\infty} A_{n}\left(C_{\lambda}\right)=L ;
$$

4. Existe um semi-espaço $H^{+}$e uma sequência de transformações lineares invertiveis $\left\{B_{n}, n \in \mathbb{N}\right\}$, tais que

$$
B_{n}\left(C_{\lambda}\right) \subset B_{n+1}\left(C_{\lambda}\right), \quad \bigcup_{n=1}^{\infty} B_{n}\left(C_{\lambda}\right)=\text { interior de } H^{+} \text {. }
$$

No que segue admitimos a existência de todas as inversas envolvidas. Particionando uma matriz $A$ como $A=\left(A_{i j}\right), i, j \in\{1, \ldots, k\}$ com cada $A_{i i}$ sendo uma matriz quadrada, façamos

$$
A_{i i . j}=A_{i i}-A_{i j} A_{j j}^{-1} A_{j i}
$$

Seja $X: p \times 1$ particionado na forma $X^{\prime}=\left(X_{1}^{\prime}, X_{2}^{\prime}\right)$, com $X_{1}: q \times 1$ e $A: p \times p$ particionada como $A=\left(A_{i j}\right), i, j=1,2$, sendo $A_{11}: q \times q$. Então,

$$
\begin{aligned}
X^{\prime} A^{-1} X & =\left(X_{1}-A_{12} A_{22}^{-1} X_{2}\right)^{\prime} A_{11.2}^{-1}\left(X_{1}-A_{12} A_{22}^{-1} X_{2}\right)+X_{2}^{\prime} A_{22}^{-1} X_{2} \\
& =\left(X_{2}-A_{21} A_{11}^{-1} X_{1}\right)^{\prime} A_{22.1}^{-1}\left(X_{2}-A_{21} A_{11}^{-1} X_{1}\right)+X_{1}^{\prime} A_{11}^{-1} X_{1} .
\end{aligned}
$$


Isto é consequência de uma partição conveniente de $A^{-1}$ (ver Mardia et al., 1979, pg. 459).

Dizemos que um vetor aleatório $X: p \times 1, p \geq 2$, tem distribuição ortogonalmente invariante quando, para toda transformação linear ortogonal $A, X$ e $A X$ têm a mesma distribuição de probabilidade.

Teorema 2.1.2 Seja $X: p \times 1$ um vetor aleatório absolutamente contínuo e com distribuição ortogonalmente invariante. Então $\|X\|^{2}$ é independente de $X /\|X\|$.

Corolário 2.1.1 Seja $X$ como no enunciado do teorema 2.1.2, seja $D$ positivamente homogêneo e seja $c \in \mathbb{R}$ arbitrário. Então os eventos $\left(\|X\|^{2} \geq c\right) e(X \in D)$ são independentes.

Demonstração: usando a hipótese de que $D$ é positivamente homogêneo, basta verificar a igualdade de conjuntos

$$
(X \in D)=\left(\frac{X}{\|X\|} \in D\right) .
$$

Como consequência imediata do corolário temos que se $X \sim N(0, \Sigma), c \in \mathbb{R}$ e $D$ é positivamente homogêneo então os eventos $\left(X^{\prime} \Sigma^{-1} X \geq c\right)$ e $(X \in D)$ são independentes. Basta observar que $\Sigma^{-1 / 2} X$ tem distribuição ortogonalmente invariante.

Estabeleceremos agora o conceito de projeção ortogonal de um ponto sobre um conjunto. Sejam $x \in \mathbb{R}^{p}$ e $D \subset \mathbb{R}^{p}$ não vazio, fixos. O conjunto $\left\{\|x-z\|_{\Sigma} ; z \in D\right\}$ é limitado inferiormente. Definimos então a distância do ponto $x$ ao conjunto $D$, $\|x-D\|_{\Sigma}$, por

$$
\|x-D\|_{\Sigma}=\inf \left\{\|x-z\|_{\Sigma} ; z \in D\right\} .
$$

Se $D$ é fechado, então sempre existe $z_{0} \in D$ que satisfaz

$$
\inf \left\{\|x-z\|_{\Sigma} ; z \in D\right\}=\left\|x-z_{0}\right\|_{\Sigma}
$$

Se $D$ é convexo e fechado, então $z_{0}$ é o único ponto em $D$ que satisfaz essa relação (ver Bazaraa \& Shetty, 1979, teorema 2.3.1). Em geral, caso exista um ponto $z_{0} \in D$ satisfazendo (2.3) diremos que este ponto é uma projeção ortogonal do ponto $x$ sobre o conjunto $D$ e o denotaremos por $\pi_{\Sigma}(x, D)$.

Teorema 2.1.3 Seja $D \subset \mathbb{R}^{p}$ positivamente homogêneo, fechado e seja $x \in \mathbb{R}^{p}$. Então, 
1. $<\pi_{\Sigma}(x, D), x-\pi_{\Sigma}(x, D)>_{\Sigma}=0$

2. $\|x\|_{\Sigma}^{2}=\left\|\pi_{\Sigma}(x, D)\right\|_{\Sigma}^{2}+\left\|x-\pi_{\Sigma}(x, D)\right\|_{\Sigma}^{2}$.

Observe que, pela unicidade do ínfimo, a função $g: \mathbb{R}^{p} \times \mathcal{M}_{p}, g(x, \Sigma)=\|x-D\|_{\Sigma}$ está bem definida. Usaremos o resultado a seguir para mostrar que essa função é contínua. Enunciaremos este lema de uma forma geral, como em Rockafellar (1970, teorema 10.7). Antes, algumas definições. Um subespaço afim de $\mathbb{R}^{p}$ é um subconjunto $M$ com a seguinte propriedade: se $x, y \in M$ então $\alpha_{1} x+\alpha_{2} y \in M$ para todos $\alpha_{1}, \alpha_{2}$ tais que $\alpha_{1}+\alpha_{2}=1$. Um subconjunto de $\mathbb{R}^{p}$ é relativamente aberto se é aberto em relação ao menor subespaço afim que o contém. Sejam $E, F$ e $G$ espaços topológicos e $g: E \times F \rightarrow G$ uma função. Diremos que $g$ é contínua em relação à primeira variável (no ponto $(a, b)$ ) quando a função parcial $g_{b}$ for contínua em $a$. Analogamente, definimos continuidade em relação à segunda variável.

Lema 2.1.1 Sejam $F \subset \mathbb{R}^{p}$ convexo e relativamente aberto e $E$ um espaço topológico localmente compacto. Seja $g: E \times F \rightarrow \mathbb{R}$ contínua em relação à primeira variável para todo $(x, y) \in E \times F$ e tal que a função parcial $g_{x}$ é convexa para todo $x \in E$. Então $g$ é contínua.

Teorema 2.1.4 Seja $D \subset \mathbb{R}^{p}$. A função distância ao conjunto $D, g: \mathbb{R}^{p} \times \mathcal{M}_{p}$, $g(x, \Sigma)=\|x-D\|_{\Sigma}$ é contínua.

Demonstração: $\mathcal{M}_{p}$ é um subconjunto aberto do espaço das matrizes simétricas de ordem $p \times p$, sendo portanto aberto em relação a qualquer subconjunto deste espaço que o contenha. Além disso, este conjunto é convexo. Obviamente $\mathbb{R}^{p}$ é um espaço topológico localmente compacto. Temos que, por definição,

$$
g(x, \Sigma)=\inf \left\{\|x-z\|_{\Sigma} ; z \in D\right\} .
$$

Como os elementos do conjunto entre chaves são não-negativos, vem que

$$
g^{2}(x, \Sigma)=\inf \left\{\|x-z\|_{\Sigma}^{2} ; z \in D\right\}
$$

(Lima, 1987, exercício 39, pg. 73). Se mostrarmos que $g^{2}$ é contínua, teremos o resultado. Observe que $g^{2}=h \circ f$, a composição de $h$ com $f$, onde $h: \mathbb{R}^{p} \times \mathcal{M}_{p} \rightarrow \mathbb{R}$ é dada por

$$
h(x, \Sigma)=\inf \left\{\|x-z\|_{\Sigma^{-1}}^{2} ; z \in D\right\},
$$


e $f: \mathbb{R}^{p} \times \mathcal{M}_{p} \rightarrow \mathbb{R}^{p} \times \mathcal{M}_{p}$ é dada por $f(x, \Sigma)=\left(x, \Sigma^{-1}\right)$. Como $f$ é contínua, basta mostrar que $h$ é contínua. Para $\Sigma>0$ fixa, a função parcial $h_{\Sigma}$ é uniformemente contínua (Lima, 1977, pg. 149, item 3). Seja $a \in \mathbb{R}^{p}$ fixo e considere a função parcial $h_{a}$. Vamos provar que $h_{a}$ é côncava. Sejam $\Sigma_{1}$ e $\Sigma_{2}$ em $\mathcal{M}_{p}$ e $\lambda \in[0,1]$. Então,

$$
\begin{aligned}
h_{a}\left(\lambda \Sigma_{1}+(1-\lambda) \Sigma_{2}\right) & =\inf \left\{\lambda\|a-z\|_{\Sigma_{1}^{-1}}^{2}+(1-\lambda)\|a-z\|_{\Sigma_{2}^{-1}}^{2} ; z \in D\right\} \\
& \geq \lambda \inf \left\{\|a-z\|_{\Sigma_{1}^{-1}}^{2} ; z \in D\right\}+(1-\lambda) \inf \left\{\|a-z\|_{\Sigma_{2}^{-1}}^{2} ; z \in D\right\} \\
& =\lambda h_{a}\left(\Sigma_{1}\right)+(1-\lambda) h_{a}\left(\Sigma_{2}\right) .
\end{aligned}
$$

Assim, $-h_{a}$ é convexa e, pelo lema, a função $-h$ é contínua, implicando em $h$ contínua.

Teorema 2.1.5 Sejam $T: \mathbb{R}^{p} \rightarrow \mathbb{R}^{p}$ uma transformação linear invertível, $D \subset \mathbb{R}^{p}$ e $x \in \mathbb{R}^{p}$ tal que existe $\pi_{\Sigma}(x, D)$. Então,

1. $T \pi_{\Sigma}(x, D)=\pi_{T \Sigma T^{\prime}}(T x, T(D))$;

2. $\left\|\pi_{\Sigma}(x, D)\right\|_{\Sigma}=\left\|\pi_{T \Sigma T^{\prime}}(T x, T(D))\right\|_{T \Sigma T^{\prime}}$;

3. $\left\|x-\pi_{\Sigma}(x, D)\right\|_{\Sigma}=\left\|T x-\pi_{T \Sigma T^{\prime}}(T x, T(D))\right\|_{T \Sigma T^{\prime}}$.

Teorema 2.1.6 Seja $\left\{D_{n}, n \in \mathbb{N}\right\}$ uma sequência de conjuntos e seja $x$ tal que existe $\pi_{\Sigma}\left(x, D_{n}\right)$ para todo $n \in \mathbb{N}$.

1. Se $D_{n} \supset D_{n+1}, n \in \mathbb{N}$, então

$$
\left\|x-\pi_{\Sigma}\left(x, D_{n}\right)\right\|_{\Sigma} \uparrow\left\|x-\pi_{\Sigma}(x, D)\right\|_{\Sigma}, \text { quando } n \rightarrow \infty
$$

onde $D=\bigcap_{n=1}^{\infty} D_{n}$;

2. Se $D_{n} \subset D_{n+1}, n \in \mathbb{N}$, então

$$
\left\|x-\pi_{\Sigma}\left(x, D_{n}\right)\right\|_{\Sigma} \downarrow\left\|x-\pi_{\Sigma}(x, D)\right\|_{\Sigma}, \text { quando } n \rightarrow \infty
$$

onde $D$ é o fecho de $\bigcup_{n=1}^{\infty} D_{n}$. 


\subsection{O Teste de Distância para Restrições de Igual- dade e Desigualdade}

Considere um modelo estatístico com família de distribuições amostrais $\left\{P_{\theta} ; \theta \in\right.$ $\Theta\}$, onde $\Theta \subset \mathbb{R}^{s}$ é aberto, e seja $\Theta^{*} \subset \Theta$. Seja $h: \Theta \rightarrow \mathbb{R}^{p}$ uma aplicação de classe $C^{1}$, com $s \geq p$. Fazendo a partição de $x \in \mathbb{R}^{p}, x^{\prime}=\left(x_{1}^{\prime}, x_{2}^{\prime}\right), x_{1}: q \times 1,0 \leq q \leq p$, defina

$$
\mathcal{A}=\left\{x \in \mathbb{R}^{p} ; x_{2} \geq 0\right\}=\mathbb{R}^{q} \times \mathcal{O}_{p-q}
$$

Suponha que

$$
\Theta^{*} \subset h^{-1}(\mathcal{A})=\{\theta \in \Theta ; h(\theta) \in \mathcal{A}\},
$$

com $\Theta^{*} \cap h^{-1}(\{0\})$ diferente de vazio. Trataremos aqui de um teste para as hipóteses

$$
H: h(\theta)=0 \text { contra } K: h(\theta) \in \mathcal{A}-\{0\}, \theta \in \Theta^{*} .
$$

Usaremos a notação $\theta \in H$ e $\theta \in K$ para indicar que o parâmetro está na hipótese nula ou alternativa, respectivamente. Considere que existe uma sequência de estatísticas em $\Theta,\left\{\bar{\theta}_{n} ; n \in \mathbb{N}\right\}$ tal que,

$$
n^{1 / 2}\left(\bar{\theta}_{n}-\theta\right) \stackrel{\mathcal{D}(\theta)}{\longrightarrow} N(0, \Omega(\theta)),
$$

para todo $\theta \in \Theta$, onde $\Omega: \Theta \rightarrow \mathcal{M}_{s}$ é uma aplicação contínua.

Denotemos por $J$ a aplicação (contínua) que associa a cada elemento de $\Theta$ a matriz Jacobiana de $h$ neste ponto. Assumiremos que $J(\theta)$ é de posto $p$ para todo $\theta \in \Theta$. Por (2.6) temos que

$$
n^{1 / 2}\left[h\left(\bar{\theta}_{n}\right)-h(\theta)\right] \stackrel{\mathcal{D}(\theta)}{\longrightarrow} N\left(0, J(\theta) \Omega(\theta) J(\theta)^{\prime}\right),
$$

para todo $\theta \in \Theta$. A matriz

$$
\Sigma(\theta)=J(\theta) \Omega(\theta) J(\theta)^{\prime}
$$

é positiva definida para todo $\theta \in \Theta$. Vamos supor a existência de uma sequência consistente de estimadores de $\Sigma(\theta),\left\{\bar{\Sigma}_{n}\right\}$, por exemplo, $\bar{\Sigma}_{n}=J\left(\bar{\theta}_{n}\right) \Omega\left(\bar{\theta}_{n}\right) J\left(\bar{\theta}_{n}\right)$. Com probabilidade $P_{\theta}$ tendendo a $1, \bar{\Sigma}_{n}$ é invertível e $\bar{\Sigma}_{n}^{-1} \stackrel{\operatorname{Pr}(\theta)}{\longrightarrow} \Sigma^{-1}(\theta)$ (Mak, 1982, lema 2.2), para todo $\theta \in \Theta$. 
Seja $\bar{\gamma}_{n}=n^{1 / 2} h\left(\bar{\theta}_{n}\right)$. Para $n \in \mathbb{N}$ fixo, a Estatística de Distância é definida por

$$
\mathrm{D}_{n}=\left\|\bar{\gamma}_{n}-\{0\}\right\|_{\bar{\Sigma}_{n}}^{2}-\left\|\bar{\gamma}_{n}-\mathcal{A}\right\|_{\bar{\Sigma}_{n}}^{2} .
$$

Pelo teorema 2.1.3 (parte 2) e observando que

$$
\mathbf{D}_{n}=\left\|\bar{\gamma}_{n}\right\|_{\bar{\Sigma}_{n}}^{2}-\left\|\bar{\gamma}_{n}-\pi_{\bar{\Sigma}_{n}}\left(\bar{\gamma}_{n}, \mathcal{A}\right)\right\|_{\bar{\Sigma}_{n}}^{2},
$$

temos

$$
\mathbf{D}_{n}=\left\|\pi_{\bar{\Sigma}_{n}}\left(\bar{\gamma}_{n}, \mathcal{A}\right)\right\|_{\bar{\Sigma}_{n}}^{2} .
$$

Obteremos agora a distribuição assintótica da estatística de distância sob $H$. Por (2.7),

$$
\left(\bar{\gamma}_{n}, \bar{\Sigma}_{n}\right) \stackrel{\mathcal{D}(\theta)}{\longrightarrow}(W, \Sigma(\theta)), \theta \in H
$$

onde $W \sim N(0, \Sigma(\theta))$. Para facilitar a notação escreveremos simplesmente $\Sigma$ em vez de $\Sigma(\theta)$. Pelo Teorema 2.1.4 resulta que

$$
\mathrm{D}_{n} \stackrel{\mathcal{D}(\theta)}{\longrightarrow}\left\|\pi_{\Sigma}(W, \mathcal{A})\right\|_{\Sigma}^{2}, \theta \in H .
$$

$\pi_{\Sigma}(W, \mathcal{A})$ é solução do problema quadrático

$$
\begin{array}{ll}
\operatorname{minimizar} & (W-z)^{\prime} \Sigma^{-1}(W-z) \\
\text { sujeito a } & z_{2} \geq 0,
\end{array}
$$

onde $z=\left(z_{1}^{\prime}, z_{2}^{\prime}\right)^{\prime}, z_{1}: q \times 1$. Sejam $f: \mathbb{R}^{p} \rightarrow \mathbb{R}, f(z)=\|W-z\|_{\Sigma}^{2}$, a função objetivo desse problema e $\Sigma=\left(\Sigma_{i j}\right), i, j \in\{1,2\}$, partição de $\Sigma$ com $\Sigma_{11}: q \times q$. Resolveremos o problema quadrático utilizando o "método do ínfimo iterado" (ver Bartle, 1976, pg. 42). Para $z_{2} \in \mathcal{O}_{p-q}$ fixo considere a função parcial $f_{z_{2}}$. Um ponto crítico de $f_{z_{2}}$ é solução da equação

$$
\partial_{1} f\left(z_{1}, z_{2}\right)=0 .
$$

Por (2.2) vemos que

$$
(W-z)^{\prime} \Sigma^{-1}(W-z)=\left\|W_{1}-z_{1}-\Sigma_{12} \Sigma_{22}^{-1}\left(W_{2}-z_{2}\right)\right\|_{\Sigma_{11.2}}^{2}+\left\|W_{2}-z_{2}\right\|_{\Sigma_{22}}^{2} .
$$

Usando esta relação e o exemplo 16 de Lima (1970) para obter a derivada parcial $\partial_{1} f\left(z_{1}, z_{2}\right)$, vem que essa equação tem como única solução o ponto

$$
\hat{z}_{1}\left(z_{2}\right)=W_{1}+\Sigma_{12} \Sigma_{22}^{-1}\left(z_{2}-W_{2}\right) .
$$


Pelo princípio do ínfimo iterado temos que

$$
\inf \left\{f\left(z_{1}, z_{2}\right) ;\left(z_{1}, z_{2}\right) \in \mathbb{R}^{q} \times \mathcal{O}_{p-q}\right\}=\inf \left\{f\left(\hat{z}_{1}\left(z_{2}\right), z_{2}\right) ; z_{2} \in \mathcal{O}_{p-q}\right\}
$$

Seja $z_{2} \in \mathcal{O}_{p-q}$. Usando (2.2) e (2.12) obtemos

$$
\begin{aligned}
f\left(\hat{z}_{1}\left(z_{2}\right), z_{2}\right) & =\left\|W_{1}-\hat{z}_{1}\left(z_{2}\right)-\Sigma_{12} \Sigma_{22}^{-1}\left(W_{2}-z_{2}\right)\right\|_{\Sigma_{11.2}}^{2}+\left\|W_{2}-z_{2}\right\|_{\Sigma_{22}}^{2} \\
& =\left\|W_{2}-z_{2}\right\|_{\Sigma_{22}}^{2},
\end{aligned}
$$

de modo que o problema reduz-se a

$$
\begin{array}{ll}
\operatorname{minimizar} & \left\|W_{2}-z_{2}\right\|_{\Sigma_{22}}^{2} \\
\text { sujeito a } & z_{2} \geq 0,
\end{array}
$$

que tem como (única) solução $\hat{z}_{2}=\pi_{\Sigma_{22}}\left(W_{2}, \mathcal{O}_{p-q}\right)$. Demonstramos o Teorema 2.2.1.

Teorema 2.2.1 $\pi_{\Sigma}(W, \mathcal{A})=\left(\hat{z}_{1}^{\prime}, \hat{z}_{2}^{\prime}\right)^{\prime}$, onde

$$
\begin{aligned}
& \hat{z}_{1}=W_{1}+\Sigma_{12} \Sigma_{22}^{-1}\left(\hat{z}_{2}-W_{2}\right) \\
& \hat{z}_{2}=\pi_{\Sigma_{22}}\left(W_{2}, \mathcal{O}_{p-q}\right) .
\end{aligned}
$$

Observe que este teorema pode ser enunciado de uma forma mais geral, substituindo $\mathcal{O}_{p-q}$ por um conjunto convexo e fechado qualquer contido em $\mathbb{R}^{p-q}$. A demonstração é exatamente a mesma.

Seja $\mathbf{D}=\left\|\pi_{\Sigma}(W, \mathcal{A})\right\|_{\Sigma}^{2}$ o limite em distribuição de $\mathbf{D}_{n}$. Usando uma representação em blocos conveniente para a matriz $\Sigma^{-1}$ obtemos o corolário 2.2.1.

\section{Corolário 2.2.1}

$$
\mathbf{D}=\left\|W_{1}-\Sigma_{12} \Sigma_{22}^{-1} W_{2}\right\|_{\Sigma_{11.2}}^{2}+\left\|\pi_{\Sigma_{22}}\left(W_{2}, \mathcal{O}_{p-q}\right)\right\|_{\Sigma_{22}}^{2} .
$$

Temos que $W_{1}-\Sigma_{12} \Sigma_{22}^{-1} W_{2} \sim N\left(0, \Sigma_{11.2}\right)$ e $W_{2} \sim N\left(0, \Sigma_{22}\right)$ são vetores aleatórios independentes (Mardia et al., 1979, teorema 3.2.3). Desta forma

$$
\left\|W_{1}-\Sigma_{12} \Sigma_{22}^{-1} W_{2}\right\|_{\Sigma_{11.2}}^{2} \sim \chi_{q}^{2}
$$

e as duas parcelas em (2.15) são independentes.

Vamos obter a distribuição de D. Seja $M \subset\{1, \ldots, p-q\}$. Uma face de $\mathcal{O}_{p-q}$ associada a $M$ é o conjunto

$$
\mathcal{X}_{M}=\left\{\left(x_{1}, \ldots, x_{p-q}\right)^{\prime} \in \mathbb{R}^{p-q} ; x_{i}>0 \text { se } i \in M \text { e } x_{i}=0 \text { se } i \notin M\right\} .
$$


Obviamente, temos um total de $2^{p-q}$ faces de $\mathcal{O}_{p-q}$. Suponha, sem perda de generalidade, que $M$ consiste dos últimos $k$ elementos de $\{1, \ldots, p-q\}$. Façamos $\nu=W_{2}$, $V=\Sigma_{22}$ e consideremos as partições $\nu=\left(\nu_{1}^{\prime}, \nu_{2}^{\prime}\right)^{\prime}$ e $V=\left(V_{i j}\right), i, j \in\{1,2\}, \nu_{2}: k \times 1$, $V_{22}: k \times k, k=0, \ldots, p-q$.

A demonstração do lema seguinte pode ser encontrada em Nüesch (1966).

\section{Lema 2.2.1 .}

1. $\left(\pi_{V}\left(\nu, \mathcal{O}_{p-q}\right) \in \mathcal{X}_{M}\right)=\left(\nu_{2}-V_{21} V_{11}^{-1} \nu_{1}>0, V_{11}^{-1} \nu_{1} \leq 0\right) ;$

2. Se $\pi_{V}\left(\nu, \mathcal{O}_{p-q}\right) \in \mathcal{X}_{M}$ então

$$
\pi_{V}\left(\nu, \mathcal{O}_{p-q}\right)=\left(\begin{array}{c}
0 \\
\nu_{2}-V_{21} V_{11}^{-1} \nu_{1}
\end{array}\right)
$$

Assim, se $\pi_{V}\left(\nu, \mathcal{O}_{p-q}\right) \in \mathcal{X}_{M}$, temos, usando (2.2), que

$$
\left\|\pi_{V}\left(\nu, \mathcal{O}_{p-q}\right)\right\|_{V}^{2}=\left\|\nu_{2}-V_{21} V_{11}^{-1} \nu_{1}\right\|_{V_{22.1}}^{2}
$$

Vamos encontrar a distribuição condicional de $\mathbf{D}$ dado o evento ${ }^{1}\left(\pi_{V}\left(\nu, \mathcal{O}_{p-q}\right) \in\right.$ $\left.\mathcal{X}_{M}\right)$. A primeira parcela em (2.15) é independente de $\nu$ e tem distribuição $\chi_{q}^{2}$. Portanto, a sua distribuição condicionada ao evento também é $\chi_{q}^{2}$. Também pode-se mostrar facilmente que as duas parcelas na expressão de $\mathbf{D}$ são condicionalmente independentes. Assim, nos resta somente determinar a distribuição de $\left\|\pi_{V}\left(\nu, \mathcal{O}_{p-q}\right)\right\|_{V}^{2}$ dado o evento $\left(\pi_{V}\left(\nu, \mathcal{O}_{p-q}\right) \in \mathcal{X}_{M}\right)$.

Temos que

$$
\begin{aligned}
P(\| & \left.\pi_{V}\left(\nu, \mathcal{O}_{p-q}\right) \|_{V}^{2} \geq c, \pi_{V}\left(\nu, \mathcal{O}_{p-q}\right) \in \mathcal{X}_{M}\right) \\
& =P\left(\left\|\nu_{2}-V_{21} V_{11}^{-1} \nu_{1}\right\|_{V_{22.1}}^{2} \geq c, \pi_{V}\left(\nu, \mathcal{O}_{p-q}\right) \in \mathcal{X}_{M}\right) \\
& =P\left(\left\|\nu_{2}-V_{21} V_{11}^{-1} \nu_{1}\right\|_{V_{22.1}}^{2} \geq c, \nu_{2}-V_{21} V_{11}^{-1} \nu_{1}>0, V_{11}^{-1} \nu_{1} \leq 0\right) \\
& =P\left(\left\|\nu_{2}-V_{21} V_{11}^{-1} \nu_{1}\right\|_{V_{22.1}}^{2} \geq c, \nu_{2}-V_{21} V_{11}^{-1} \nu_{1}>0\right) P\left(V_{11}^{-1} \nu_{1} \leq 0\right) \\
& =P\left(\left\|\nu_{2}-V_{21} V_{11}^{-1} \nu_{1}\right\|_{V_{22.1}}^{2} \geq c\right) P\left(\nu_{2}-V_{21} V_{11}^{-1} \nu_{1}>0\right) P\left(V_{11}^{-1} \nu_{1} \leq 0\right) .
\end{aligned}
$$

Como $\nu \sim N(0, V)$, temos que $\nu_{2}-V_{21} V_{11}^{-1} \nu_{1}$ e $\nu_{1}$ são independentes, o que justifica a terceira igualdade em (2.16). Como $\nu_{2}-V_{21} V_{11}^{-1} \nu_{1} \sim N\left(0, V_{22.1}\right)$ resulta, pela

\footnotetext{
${ }^{1} \mathrm{Ou}$ seja, a projeção de $\nu$ sobre $\mathcal{O}_{p-q}$ possui as $p-q-k$ primeiras coordenadas nulas e as $k$ restantes positivas.
} 
observação que segue o Corolário 2.1.1, que $\left(\left\|\nu_{2}-V_{21} V_{11}^{-1} \nu_{1}\right\|_{V_{22.1}}^{2} \geq c\right)$ e $\left(\nu_{2}-\right.$ $\left.V_{21} V_{11}^{-1} \nu_{1}>0\right)$ são eventos independentes, justificando a quarta igualdade.

Assim,

$$
\begin{aligned}
P(\| & \left.\pi_{V}\left(\nu, \mathcal{O}_{p-q}\right) \|_{V}^{2} \geq c \mid \pi_{V}\left(\nu, \mathcal{O}_{p-q}\right) \in \mathcal{X}_{M}\right) \\
& =\frac{P\left(\left\|\nu_{2}-V_{21} V_{11}^{-1} \nu_{1}\right\|_{V_{22.1}}^{2} \geq c\right) P\left(\nu_{2}-V_{21} V_{11}^{-1} \nu_{1}>0\right) P\left(V_{11}^{-1} \nu_{1} \leq 0\right)}{P\left(\nu_{2}-V_{21} V_{11}^{-1} \nu_{1}>0\right) P\left(V_{11}^{-1} \nu_{1} \leq 0\right)} \\
& =P\left(\left\|\nu_{2}-V_{21} V_{11}^{-1} \nu_{1}\right\|_{V_{22.1}}^{2} \geq c\right) \\
& =P\left(\chi_{k}^{2} \geq c\right) .
\end{aligned}
$$

Ou seja, a distribuição condicional de $\mathbf{D}$ dado o evento $\left(\pi_{V}\left(\nu, \mathcal{O}_{p-q}\right) \in \mathcal{X}_{M}\right)$ é $\chi_{q+k}^{2}$. Então,

$$
\begin{aligned}
P(\mathrm{D} \geq c) & =\sum_{M} P\left(\mathbf{D} \geq c \mid \pi_{V}\left(\nu, \mathcal{O}_{p-q}\right) \in \mathcal{X}_{M}\right) P\left(\pi_{V}\left(\nu, \mathcal{O}_{p-q}\right) \in \mathcal{X}_{M}\right) \\
& =\sum_{k=0}^{p-q} \sum_{\{M ; \# M=k\}} P\left(\chi_{q+k}^{2} \geq c\right) P\left(\pi_{V}\left(\nu, \mathcal{O}_{p-q}\right) \in \mathcal{X}_{M}\right) \\
& =\sum_{k=0}^{p-q} P\left(\chi_{q+k}^{2} \geq c\right) w\left(p-q, k, \Sigma_{22}\right)
\end{aligned}
$$

onde

$$
w\left(p-q, k, \Sigma_{22}\right)=\sum_{\{M ; \# M=k\}} P\left(\pi_{\Sigma_{22}}\left(W_{2}, \mathcal{O}_{p-q}\right) \in \mathcal{X}_{M}\right)
$$

ou seja, $w\left(p-q, k, \Sigma_{22}\right)$ é a probabilidade de que a projeção (segundo a norma induzida por $\Sigma_{22}$ ) de $W_{2}$ sobre $\mathcal{O}_{p-q}$ tenha exatamente $k$ coordenadas positivas. Assim, a distribuição nula limite de $\mathbf{D}_{n}$ é uma mistura de funções de distribuição qui-quadrado com pesos dados por (2.17). A rigor, o cálculo destes pesos resume-se ao problema de calcular a probabilidade de que as coordenadas de um vetor aleatório com distribuição normal multivariada sejam todas positivas. Para ver isto, tome $M=\left\{i_{1}, \ldots, i_{k}\right\} \subset\{1, \ldots, p-q\}$, particione $\nu=W_{2}$ fazendo $\nu=\left(\nu_{1}^{\prime}, \nu_{2}^{\prime}\right)^{\prime}$, onde $\nu_{2}=$ $\left(\nu_{i_{1}}, \ldots, \nu_{i_{k}}\right)^{\prime}$ e $\nu_{1}$ é formado pelas coordenadas restantes, e use o Lema 2.2 .1 para obter cada parcela em (2.17). Em algumas situações, como quando $\Sigma_{22}$ é diagonal, ou quando $p-q \leq 4$, existem expressões analíticas para os pesos, ver o Apêndice B. Para $p-q \geq 5$, temos procedimentos computacionais como os apresentados em Bohrer \& Chow (1978) e Sun (1988a,b).

Para referências futuras, enunciamos o Teorema 2.2.2. 
Teorema 2.2.2 Seja $\theta \in H, \Sigma(\theta)$ definida em (2.8) e considere a partição $\Sigma(\theta)=$ $\left(\Sigma_{i j}(\theta)\right), i, j=1,2, \Sigma_{22}(\theta):(p-q) \times(p-q)$. Então,

$$
\lim _{n \rightarrow \infty} P_{\theta}\left(\mathbf{D}_{n} \geq c\right)=\sum_{k=0}^{p-q} P\left(\chi_{q+k}^{2} \geq c\right) w\left(p-q, k, \Sigma_{22}(\theta)\right)
$$

onde $w\left(p-q, k, \Sigma_{22}(\theta)\right)$ é a probabilidade de que a projeção (segundo a norma induzida por $\left.\Sigma_{22}(\theta)\right)$ de um vetor com distribuição $N_{p-q}\left(0, \Sigma_{22}(\theta)\right)$ sobre $\mathcal{O}_{p-q}$ tenha exatamente $k$ coordenadas positivas.

Mostraremos agora como definir um teste de nível aproximado $\alpha \in(0,1)$ usando as idéias apresentadas. Considere a seguinte função definida no conjunto $\mathbb{R}^{+} \times \mathcal{M}_{p}$,

$$
\phi(c, \Sigma)=P\left(\left\|\pi_{\Sigma}(X, A)\right\|_{\Sigma}^{2} \geq c\right)
$$

onde $X \sim N(0, \Sigma)$ (a distribuição limite no Teorema 2.2 .2 é a função $\phi_{\Sigma(\theta)}$, para $\theta$ em $H$ ). Considere as soluções em $c$ da equação

$$
\sup \{\phi(c, \Sigma(\theta)) ; \theta \in H\}=\alpha .
$$

O valor crítico para o teste de distância de nível $\alpha$ é o mínimo (se existir) do conjunto formado por estas soluções. Vamos denotá-lo por $c^{*}(\alpha)$. Supondo que $\theta \in H$ temos que, quando $n \rightarrow \infty$,

$$
P_{\theta}\left(\mathbf{D}_{n}>c^{*}(\alpha)\right) \rightarrow \phi\left(c^{*}(\alpha), \Sigma(\theta)\right) \leq \sup \left\{\phi\left(c^{*}(\alpha), \Sigma(\theta)\right) ; \theta \in H\right\}=\alpha .
$$

A implementação deste teste pode apresentar muitas dificuldades computacionais, por causa do cálculo de $c^{*}(\alpha)$. Discutiremos agora algumas maneiras de contorná-las. A primeira sugestão é consequência do Teorema 2.2.3.

\section{Teorema 2.2.3 Seja $c \in \mathbb{R}$. Então}

$$
\begin{aligned}
\inf \{\phi(c, \Sigma) ; \Sigma>0\} & =\frac{1}{2} P\left(\chi_{q}^{2} \geq c\right)+\frac{1}{2} P\left(\chi_{q+1}^{2} \geq c\right) ; \\
\sup \{\phi(c, \Sigma) ; \Sigma>0\} & =\frac{1}{2} P\left(\chi_{p-1}^{2} \geq c\right)+\frac{1}{2} P\left(\chi_{p}^{2} \geq c\right) .
\end{aligned}
$$

Antes da demonstração, mostraremos como este teorema pode ser útil na implementação do teste. É simples obter $c_{1}(\alpha)$ e $c_{2}(\alpha)$ tais que

$$
\begin{aligned}
& \frac{1}{2} P\left(\chi_{q}^{2} \geq c_{1}(\alpha)\right)+\frac{1}{2} P\left(\chi_{q+1}^{2} \geq c_{1}(\alpha)\right)=\alpha \\
& \frac{1}{2} P\left(\chi_{p-1}^{2} \geq c_{2}(\alpha)\right)+\frac{1}{2} P\left(\chi_{p}^{2} \geq c_{2}(\alpha)\right)=\alpha .
\end{aligned}
$$


Temos que $c_{1}(\alpha) \leq c^{*}(\alpha) \leq c_{2}(\alpha)$. Com efeito, se fosse $c_{2}(\alpha)<c^{*}(\alpha)$ teríamos, usando o fato que a função $c \mapsto \frac{1}{2} P\left(\chi_{p-1}^{2} \geq c\right)+\frac{1}{2} P\left(\chi_{p}^{2} \geq c\right), c>0$, é estritamente decrescente,

$$
\begin{aligned}
\alpha & =\sup \left\{\phi\left(c^{*}(\alpha), \Sigma(\theta)\right) ; \theta \in H\right\} \leq \sup \left\{\phi\left(c^{*}(\alpha), \Sigma\right) ; \Sigma>0\right\} \\
& <\sup \left\{\phi\left(c_{2}(\alpha), \Sigma\right) ; \Sigma>0\right\}=\alpha,
\end{aligned}
$$

absurdo. Analogamente podemos provar que $c_{1}(\alpha) \leq c^{*}(\alpha)$. Ou seja, $\mathbf{D}_{n}>c_{2}(\alpha)$ implica em rejeição de $H$, enquanto $\mathbf{D}_{n}<c_{1}(\alpha)$ implica em sua aceitação. Assim, se uma destas situações ocorrer, não é necessária a obtenção de $c^{*}(\alpha)$. No entanto, nada podemos concluir caso ocorra $c_{1}(\alpha) \leq \mathbf{D}_{n} \leq c_{2}(\alpha)$.

Demonstração do Teorema 2.2.3: Assumiremos que $p-q \geq 1$ pois, para $p=q$ temos $\mathcal{A}=\mathbb{R}^{p}$ e a estatística de distância é igual a estatística do teste de Wald usual (Wald, 1943) para as hipóteses $h(\theta)=0$ contra $h(\theta) \in \mathbb{R}^{p}$, cuja distribuição assintótica sob a hipótese nula não depende de $\Sigma$.

Seja $\Sigma>0$ arbitrária e considere o semi-espaço

$$
H^{+}=\left\{\left(x_{1}, \ldots, x_{p}\right)^{\prime} ; x_{p} \geq 0\right\}=\mathbb{R}^{p-1} \times \mathcal{O}_{1} .
$$

Temos que $\mathcal{A} \subset H^{+}$e, portanto,

$$
\left\|\pi_{\Sigma}(X, \mathcal{A})\right\|_{\Sigma}^{2} \leq\left\|\pi_{\Sigma}\left(X, H^{+}\right)\right\|_{\Sigma}^{2}
$$

Assim,

$$
P\left(\left\|\pi_{\Sigma}(X, \mathcal{A})\right\|_{\Sigma}^{2} \geq c\right) \leq P\left(\left\|\pi_{\Sigma}\left(X, H^{+}\right)\right\|_{\Sigma}^{2} \geq c\right) .
$$

Fazendo $q=p-1$ no teorema 2.2 .2 , temos

$$
\begin{aligned}
P\left(\left\|\pi_{\Sigma}\left(X, H^{+}\right)\right\|_{\Sigma}^{2} \geq c\right) & =P\left(\chi_{p-1}^{2} \geq c\right) w\left(1,0, \Sigma_{22}\right)+P\left(\chi_{p}^{2} \geq c\right) w\left(1,1, \Sigma_{22}\right) \\
& =\frac{1}{2} P\left(\chi_{p-1}^{2} \geq c\right)+\frac{1}{2} P\left(\chi_{p}^{2} \geq c\right)
\end{aligned}
$$

Seja $\beta=\frac{1}{2} P\left(\chi_{p-1}^{2} \geq c\right)+\frac{1}{2} P\left(\chi_{p}^{2} \geq c\right)$. Provamos que $\phi_{c}(\Sigma) \leq \beta$ para toda $\Sigma>0$, de maneira que

$$
\sup \left\{\phi_{c}(\Sigma) ; \Sigma>0\right\} \leq \beta .
$$

Exibiremos agora uma sequência $\left\{\Sigma_{m} ; m \in \mathbb{N}\right\}$, com $\Sigma_{m}>0, m \in \mathbb{N}$, de tal forma que

$$
\phi_{c}\left(\Sigma_{m}\right) \rightarrow \beta \text { quando } m \rightarrow \infty
$$


Como $\mathcal{O}_{p-q}$ é um cone de interior não vazio existem, pelo teorema 2.1.1, um cone circular à direita $C_{\nu}, \nu \in(0,1)$, um semi-espaço $J^{+}$e uma sequência de matrizes invertíveis, $\left\{B_{m} ; m \in \mathbb{N}\right\}$ tais que $C_{\nu} \subset \mathcal{O}_{p-q}$ e

$$
B_{m}\left(C_{\nu}\right) \subset B_{m+1}\left(C_{\nu}\right), \quad \bigcup_{m=1}^{\infty} B_{m}\left(C_{\nu}\right)=\text { interior de } J^{+} \text {. }
$$

$B_{m}\left(C_{\nu}\right)$ é um cone para todo $m \in \mathbb{N}$ e $J^{+}$é fechado. Seja $B: q \times q$ invertível. Para cada $m$ natural defina

$$
T_{m}=\left(\begin{array}{cc}
B & 0 \\
0 & B_{m}
\end{array}\right), \quad \Sigma_{m}=\left(\begin{array}{cc}
\left(B^{\prime} B\right)^{-1} & 0 \\
0 & \left(B_{m}^{\prime} B_{m}\right)^{-1}
\end{array}\right),
$$

e tome $X_{m} \sim N\left(0, \Sigma_{m}\right)$. Observe que $T_{m} X_{m} \sim N(0, I)$. Assim,

$$
\begin{aligned}
\beta \geq \phi_{c}\left(\Sigma_{m}\right) & =P\left(\left\|\pi_{\Sigma_{m}}\left(X_{m}, \mathcal{A}\right)\right\|_{\Sigma_{m}}^{2} \geq c\right) \\
& =P\left(\left\|\pi_{\Sigma_{m}}\left(X_{m}, \mathbb{R}^{q} \times \mathcal{O}_{p-q}\right)\right\|_{\Sigma_{m}}^{2} \geq c\right) \\
& =P\left(\left\|\pi_{I}\left(T_{m} X_{m}, T_{m}\left(\mathbb{R}^{q} \times \mathcal{O}_{p-q}\right)\right)\right\|^{2} \geq c\right) \\
& =P\left(\left\|\pi_{I}\left(Z, \mathbb{R}^{q} \times B_{m}\left(\mathcal{O}_{p-q}\right)\right)\right\|^{2} \geq c\right) \\
& =P\left(\left\|Z_{1}\right\|_{I}^{2}+\left\|\pi_{I}\left(Z_{2}, B_{m}\left(\mathcal{O}_{p-q}\right)\right)\right\|^{2} \geq c\right) \\
& \geq P\left(\left\|Z_{1}\right\|_{I}^{2}+\left\|\pi_{I}\left(Z_{2}, B_{m}\left(C_{\nu}\right)\right)\right\|^{2} \geq c\right),
\end{aligned}
$$

onde $Z=\left(Z_{1}^{\prime}, Z_{2}^{\prime}\right)^{\prime} \sim N(0, I), Z_{1}: q \times 1$. A igualdade na terceira linha vale pela segunda parte do Teorema 2.1.5. A igualdade na quinta linha segue pelo Corolário 2.2.1, com as devidas adaptações. Seja $T$ uma transformação linear ortogonal tal que $^{2}$

$$
T\left(J^{+}\right)=\left\{\left(x_{1}, \ldots, x_{p-q}\right) \in \mathbb{R}^{p-q} ; x_{p-q} \geq 0\right\} .
$$

Então,

$$
\begin{aligned}
\left.P\left(\left\|Z_{1}\right\|_{I}^{2}+\| \pi_{I}\left(Z_{2}, J^{+}\right)\right) \|_{I}^{2} \geq c\right) & \left.=P\left(\left\|Z_{1}\right\|_{I}^{2}+\| \pi_{I}\left(Z_{2}, T\left(J^{+}\right)\right)\right) \|_{I}^{2} \geq c\right) \\
& \left.=P\left(\| \pi_{I}\left(Z, \mathbb{R}^{q} \times T\left(J^{+}\right)\right)\right) \|_{I}^{2} \geq c\right) \\
& \left.=P\left(\| \pi_{I}\left(Z, H^{+}\right)\right) \|_{I}^{2} \geq c\right)=\beta,
\end{aligned}
$$

pois $Z_{2}$ tem distribuição ortogonalmente invariante. Pelo Teorema 2.1.6,

$$
P\left(\left\|Z_{1}\right\|_{I}^{2}+\left\|\pi_{I}\left(Z_{2}, B_{m}\left(C_{\nu}\right)\right)\right\|_{I}^{2} \geq c\right) \rightarrow P\left(\left\|Z_{1}\right\|_{I}^{2}+\left\|\pi_{I}\left(Z_{2}, J^{+}\right)\right\|_{I}^{2} \geq c\right),
$$

\footnotetext{
${ }^{2}$ Seja $J^{+}=\left\{x \in \mathbb{R}^{p-q} ; a^{\prime} x \geq 0\right\}$. Tome $T$ ortogonal tal que $T(a)$ é igual ao $(p-q)$-ésimo vetor da base canônica de $\mathbb{R}^{p-q}$.
} 
quando $m \rightarrow \infty$. Assim,

$$
\phi_{c}\left(\Sigma_{m}\right) \rightarrow \beta
$$

quando $m \rightarrow \infty$. Como

$$
\phi_{c}\left(\Sigma_{m}\right) \leq \sup \left\{\phi_{c}(\Sigma) ; \Sigma>0\right\} \leq \beta
$$

para todo $m \in \mathbb{N}$ vem que

$$
\sup \left\{\phi_{c}(\Sigma) ; \Sigma>0\right\}=\beta .
$$

Para determinarmos o ínfimo o procedimento é semelhante. Considere novamente $\Sigma>0$ arbitrária, $X \sim N(0, \Sigma)$, e o subconjunto de $\mathbb{R}^{p-q}, L=\{0\} \times \mathcal{O}_{1}$. Então $\mathbb{R}^{q} \times L \subset \mathcal{A}$ e assim

$$
P\left(\left\|\pi_{\Sigma}\left(X, \mathbb{R}^{q} \times L\right)\right\|_{\Sigma}^{2} \geq c\right) \leq P\left(\left\|\pi_{\Sigma}(X, \mathcal{A})\right\|_{\Sigma}^{2} \geq c\right) .
$$

Observe que, pelo comentário que segue o Teorema 2.2.1,

$$
\left\|\pi_{\Sigma}\left(X, \mathbb{R}^{q} \times L\right)\right\|_{\Sigma}^{2}=\left\|X_{1}-\Sigma_{12} \Sigma_{22}^{-1} X_{2}\right\|_{\Sigma_{11,2}}^{2}+\left\|\pi_{\Sigma_{22}}\left(X_{2}, L\right)\right\|_{\Sigma_{22}}^{2} .
$$

Fazendo $U=\Sigma_{22}, u=\left(u_{1}^{\prime}, u_{2}^{\prime}\right)^{\prime}=X_{2}, u_{1}:(p-q-1) \times 1$, usando o princípio do ínfimo iterado e (2.2) obtemos

$$
\pi_{U}(u, L)=\left(\begin{array}{c}
0 \\
\pi_{U_{22.1}}\left(u_{2}-U_{21} U_{11}^{-1} u_{1}, \mathcal{O}_{1}\right)
\end{array}\right),
$$

de modo que

$$
\left\|\pi_{\Sigma}\left(X, \mathbb{R}^{q} \times L\right)\right\|_{\Sigma}^{2}=\left\|X_{1}-\Sigma_{12} \Sigma_{22}^{-1} X_{2}\right\|_{\Sigma_{11.2}}^{2}+\left\|\pi_{U_{22.1}}\left(u_{2}-U_{21} U_{11}^{-1} u_{1}, \mathcal{O}_{1}\right)\right\|_{U_{22.1}} .
$$

Esta é uma expressão semelhante a (2.15) e o mesmo raciocínio que levou à demonstração do Teorema 2.2.2 pode ser usado aqui, com 1 no lugar de $p-q$ e $u_{2}$ no lugar de $W_{2}$, para obtermos

$$
P\left(\left\|\pi_{\Sigma}\left(X, \mathbb{R}^{q} \times L\right)\right\|_{\Sigma}^{2} \geq c\right)=\frac{1}{2} P\left(\chi_{q}^{2} \geq c\right)+\frac{1}{2} P\left(\chi_{q+1}^{2} \geq c\right) \equiv \gamma .
$$

Por (2.22),

$$
\inf \left\{\phi_{c}(\Sigma) ; \Sigma>0\right\} \geq \gamma
$$


Pelo teorema 2.1.1 existem um cone circular à direita $C_{\phi}, \phi \in(0,1)$, uma semireta $L^{*}$ e uma sequência $\left\{F_{m} ; m \in \mathbb{N}\right\}$ de transformações lineares invertíveis tais que $\mathcal{O}_{p-q} \subset C_{\phi} \mathrm{e}$

$$
F_{m}\left(C_{\phi}\right) \supset F_{m+1}\left(C_{\phi}\right), \quad \bigcap_{m=1}^{\infty} F_{m}\left(C_{\phi}\right)=L .
$$

Definindo uma sequência de matrizes $\Sigma_{m}^{*}$ de maneira análoga à definição de $\Sigma_{m}$ em (2.21), com $F_{m}$ no lugar de $B_{m}$, pode-se demonstrar de maneira semelhante à que foi feita anteriormente que

$$
\phi_{c}\left(\Sigma_{m}^{*}\right) \rightarrow \gamma
$$

quando $m \rightarrow \infty$ e isto implica em

$$
\inf \left\{\phi_{c}(\Sigma) ; \Sigma>0\right\}=\gamma .
$$

Um procedimento alternativo para testar $H$ contra $K$ é baseado no próximo teorema. Defina $c_{\alpha}: \Theta \rightarrow \mathbb{R}$ da seguinte maneira

$$
\phi\left(c_{\alpha}(\theta), \Sigma(\theta)\right)=\alpha .
$$

Teorema 2.2.4 Suponha que a função $\theta \mapsto \Sigma(\theta), \theta \in \Theta$ é de classe $C^{1}$. Então, para cada $\theta_{0} \in \Theta$ existe $N_{0}$, vizinhança aberta de $\theta_{0}$, tal que $\left.c_{\alpha}\right|_{N_{0}}$ é uma função de classe $C^{1}$.

Demonstração: Os pesos que aparecem na expressão da função de distribuição limite de $\mathbf{D}_{n}$ são somas de probabilidades que são obtidas integrando-se uma densidade normal multivariada em um boreliano do espaço Euclideano (ver 2.17 e o lema 2.2.1). Assim, por Lehmann (1986, teorema 9, pg. 59), resulta que a função $\theta \mapsto w\left(p-q, k, \Sigma_{22}(\theta)\right), \theta \in \Theta$ é de classe $C^{1}$. Como uma função de distribuição qui-quadrado é infinitamente diferenciável nos reais positivos, resulta que $f(c, \theta)=\phi(c, \Sigma(\theta)),(c, \theta) \in \mathbb{R}^{+} \times \Theta$ é de classe $C^{1}$. $f_{\theta_{0}}$ é igual a um menos a restrição de uma mistura de funções de distribuição qui-quadrado ao conjunto $\mathbb{R}^{+}$. Assim, $f_{\theta_{0}}$ é estritamente decrescente. Então existe um único $c_{0}>0$ tal que $f\left(c_{0}, \theta_{0}\right)=\alpha$. Temos também que a derivada de $f_{\theta_{0}}$ é não nula para todo real positivo. Pelo Teorema da Função Implícita (Bartle, 1976, cap. 7), existe uma única função de classe $C^{1}$, $\xi: N_{0} \rightarrow \mathbb{R}$, onde $N_{0}$ é uma vizinhança de $\theta_{0}$, tal que $c_{0}=\xi\left(\theta_{0}\right)$ e $\phi(\xi(\theta), \theta)=\alpha$ para todo $\theta \in N_{0}$. Obviamente, $\xi=\left.c_{\alpha}\right|_{N_{0}}$. 
Assim, $c_{\alpha}\left(\bar{\theta}_{n}\right) \stackrel{\operatorname{Pr}(\theta)}{\longrightarrow} c_{\alpha}(\theta), \theta \in \Theta$. Seja $\theta \in H$. Pelo Teorema de Slutsky, $\mathrm{D}_{n}-$ $c_{\alpha}\left(\bar{\theta}_{n}\right) \stackrel{\mathcal{D}(\theta)}{\longrightarrow} \mathrm{D}-c_{\alpha}(\theta)$, onde $\mathbf{D}=\left\|\pi_{\Sigma(\theta)}(X, \mathcal{A})\right\|_{\Sigma(\theta)}^{2}, X \sim N(0, \Sigma(\theta))$. Como o único possível ponto de descontinuidade da função de distribuição de $\mathbf{D}-c_{\alpha}(\theta)$ é $-c_{\alpha}(\theta)<0$, vem que

$$
P_{\theta}\left(\mathbf{D}_{n}-c_{\alpha}\left(\bar{\theta}_{n}\right) \leq 0\right) \rightarrow P\left(\mathbf{D}-c_{\alpha}(\theta) \leq 0\right)=1-\alpha
$$

quando $n \rightarrow \infty$. Portanto, o teste definido por: rejeitar $H$ se e somente $\mathbf{D}_{n}>c_{\alpha}\left(\bar{\theta}_{n}\right)$ é de nível aproximado $\alpha$.

O Teorema 2.2.3 também é aplicável na implementação deste último teste. Para ver isto, observe que para $\theta \in \Theta$,

$$
\phi\left(c_{2}(\alpha), \Sigma(\theta)\right) \leq \sup \left\{\phi\left(c_{2}(\alpha), \Sigma\right) ; \Sigma>0\right\}=\alpha=\phi\left(c_{\alpha}(\theta), \Sigma(\theta)\right) .
$$

Como $\phi_{\Sigma(\theta)}$ é estritamente decrescente, resulta que $c_{\alpha}(\theta) \leq c_{2}(\alpha)$. Analogamente, provamos que $c_{1}(\alpha) \leq c_{\alpha}(\theta)$. Estas desigualdades valem para todo $\theta \in \Theta$ e, em particular, para $\left\{\bar{\theta}_{n}\right\}$. Novamente, temos que $\mathbf{D}_{n}>c_{2}(\alpha)$ implica em rejeição de $H$, enquanto $\mathbf{D}_{n}<c_{1}(\alpha)$ implica em sua aceitação.

Alguns comentários finais em relação ao poder assintótico do teste de distância e à obtenção do valor da estatística de teste. Sobre o poder: suponha que $h(\theta)$ esteja no interior do conjunto $\mathcal{A}$. Seja

$$
\mathbf{Q}_{n}=n\left[h\left(\bar{\theta}_{n}\right)\right]^{\prime} \Sigma^{-1}\left(\bar{\theta}_{n}\right) h\left(\bar{\theta}_{n}\right)
$$

a estatística de Wald (Wald, 1943). Como

$$
P_{\theta}\left(h\left(\bar{\theta}_{n}\right) \in \mathcal{A}\right)=P_{\theta}\left(\mathbf{D}_{n}=\mathbf{Q}_{n}\right),
$$

temos que

$$
\lim _{n \rightarrow \infty} P_{\theta}\left(\mathbf{D}_{n}=\mathbf{Q}_{n}\right)=1
$$

Por (210) em Wald (1943),

$$
\lim _{n \rightarrow \infty} P_{\theta}\left(\mathbf{Q}_{n}<c\right)-F_{p}\left[\lambda_{n}^{2}(\theta), c\right]=0,
$$

onde $F_{p}\left[\lambda_{n}^{2}(\theta), \cdot\right]$ é a função de distribuição qui-quadrado não central com $p$ graus de liberdade e parâmetro de não centralidade $\lambda_{n}^{2}(\theta)=n[h(\theta)]^{\prime} \Sigma^{-1}(\theta) h(\theta)$. (2.26) e (2.27) implicam em

$$
\lim _{n \rightarrow \infty} P_{\theta}\left(\mathbf{D}_{n}<c\right)-F_{p}\left[\lambda_{n}^{2}(\theta), c\right]=0
$$


Seja $\theta_{0} \in H$ e considere uma sequência $\left\{\theta_{n}\right\}$ de pontos no espaço paramétrico tal que $\theta_{n} \rightarrow \theta_{0}$ e $h\left(\theta_{n}\right)=n^{-1 / 2} \delta, \delta>0$. Se a convergência em (2.28) é uniforme, temos

$$
\lim _{n \rightarrow \infty} P_{\theta_{n}}\left(\mathbf{D}_{n}<c\right)-F_{p}\left[\delta^{\prime} \Sigma^{-1}\left(\theta_{0}\right) \delta, c\right]=0 .
$$

Para $p=1$ temos que, para um mesmo nível, o valor crítico do teste de distância é menor que o do teste de Wald. Então o teste de distância é assintoticamente mais eficiente que o teste de Wald, no sentido de Pittman (Rao, 1973, pg. 465).

A determinação do valor da estatística de distância depende da solução do problema

$$
\begin{array}{ll}
\text { minimizar } & (z-x)^{\prime} A(z-x) \\
\text { sujeito a } & x \geq 0
\end{array}
$$

onde $z$ é um vetor fixo do espaço Euclideano e $A$ é uma matriz positiva definida — veja o Teorema 2.2.1. Em Programação Não-Linear, problemas desta classe são conhecidos como problemas de programação quadrática. A teoria relevante e os métodos (algoritmos) para resolução podem ser encontrados em textos como Bazaraa \& Shetty (1979) e Luenberger (1984). No apêndice A temos uma pequena descrição do método que utilizamos nas aplicações que virão a seguir. 


\section{Capítulo 3}

\section{Testes para Hipóteses Restritas em Modelos de Regressão Linear Simples com Erros nas Variáveis}

Neste capítulo aplicamos a teoria geral do teste de distância, desenvolvida no capítulo 2, a modelos de regressão linear simples com erros nas variáveis. Uma extensão para $k \geq 2$ populações independentes também é apresentada. Mostramos, através da análise de funções de poder empíricas, obtidas por simulações em computador, que alguns testes existentes na literatura têm uma performance inferior à do teste de distância, certamente por não terem sido delineados especificamente para a situação envolvendo erros nas variáveis/hipóteses restritas.

\subsection{Alguns Modelos de Regressão com Erros nas Variáveis}

\subsubsection{Modelo Geral}

O modelo de regressão linear simples com erros nas variáveis é definido da seguinte maneira: sejam $\left(x_{i}, y_{i}\right)^{\prime}, i \in \mathbb{N}$ vetores aleatórios bidimensionais satisfazendo

$$
y_{i}=\alpha+\beta x_{i}, \quad i \in \mathbb{N},
$$

onde $\alpha, \beta \in \mathbb{R}$ são parâmetros a serem estimados. Suponha que $x_{i}$ e $y_{i}$ não são observáveis, e o que realmente podemos observar são os seus valores mais um erro 
aleatório. Ou seja, as observações são da forma

$$
\left(X_{i}, Y_{i}\right)^{\prime}=\left(x_{i}, y_{i}\right)^{\prime}+\left(\delta_{i}, \epsilon_{i}\right)^{\prime}, i \in \mathbb{N} .
$$

Vamos assumir que

$$
\left\{\left(x_{i}, \delta_{i}, \epsilon_{i}\right)^{\prime}, i \in \mathbb{N}\right\} \sim N I I D\left[(\mu, 0,0)^{\prime}, \operatorname{diag}\left\{\sigma_{x}^{2}, \sigma_{\delta}^{2}, \sigma_{\epsilon}^{2}\right\}\right],
$$

onde $\mu, \sigma_{x}^{2}, \sigma_{\delta}^{2}$ e $\sigma_{\epsilon}^{2}$ são desconhecidos e a notação $\sim N I I D$ indica que os vetores no conjunto em questão são independentes e possuem todos a mesma distribuição normal, com a média e a matriz de covariâncias indicadas.

Por (3.1), (3.2) e (3.3) temos que

$$
\left\{\left(X_{i}, Y_{i}\right)^{\prime}, i \in \mathbb{N}\right\} \sim N I I D\left[(\mu, \alpha+\beta \mu)^{\prime},\left(\begin{array}{cc}
\sigma_{x}^{2}+\sigma_{\delta}^{2} & \beta \sigma_{x}^{2} \\
\beta \sigma_{x}^{2} & \beta^{2} \sigma_{x}^{2}+\sigma_{\epsilon}^{2}
\end{array}\right)\right] .
$$

Isto define um modelo estatístico mas, para os nossos propósitos, algumas restrições adicionais são necessárias. Primeiramente, observe que a matriz de covariâncias de $\left(X_{1}, Y_{1}\right)^{\prime}$ é singular para alguns valores das coordenadas do parâmetro $\theta=\left(\mu, \alpha, \beta, \sigma_{x}^{2}, \sigma_{\delta}^{2}, \sigma_{\epsilon}^{2}\right)^{\prime}$ como, por exemplo, $\sigma_{x}^{2}=\sigma_{\delta}^{2}=0 \mathrm{e}$, nestes casos, a distribuição de $\left(X_{1}, Y_{1}\right)^{\prime}$ não tem densidade. Esta matriz, que denotaremos por $V(\theta)$, é positiva definida se e somente se pelo menos duas entre as variâncias $\sigma_{x}^{2}$, $\sigma_{\delta}^{2}$ e $\sigma_{\epsilon}^{2}$ são positivas e $\beta \neq 0$ se $\sigma_{\epsilon}^{2}=0$. Imporemos então estas restrições ao espaço paramétrico. Uma outra característica indesejável é que o modelo não é identificável: os pontos $\theta^{*}=(1,1,1,1,1,1)^{\prime}$ e $\theta^{* *}=(1,1.5,0.5,2,0,1.5)^{\prime}$ levam à mesma distribuição

$$
N\left[(1,2)^{\prime},\left(\begin{array}{ll}
2 & 1 \\
1 & 2
\end{array}\right)\right]
$$

em (3.4), por exemplo. Suposições que tornam o modelo identificável são apresentadas nas duas subseções seguintes.

\subsubsection{Modelo Passando Pela Origem}

Considere (3.1), (3.2) e (3.3), com $\alpha$ conhecido. Sem perda de generalidade podemos assumir que $\alpha=0 \mathrm{e}$, por esta razão, usaremos a denominação modelo passando pela origem (MPO) para o modelo obtido. Assim, temos que

$$
\left\{\left(X_{i}, Y_{i}\right)^{\prime}, i \in \mathbb{N}\right\} \sim N I I D\left[(\mu, \beta \mu)^{\prime}, V(\theta)\right]
$$


onde $\theta=\left(\mu, \beta, \sigma_{x}^{2}, \sigma_{\delta}^{2}, \sigma_{\epsilon}^{2}\right)^{\prime}$ e $V(\theta)$ é definida como anteriormente. Para garantir a identificabilidade do modelo definido pela amostra em (3.5) devemos ter $\mu \neq 0$ e $\beta \neq 0$, de modo que definimos como espaço paramétrico associado ao MPO o conjunto

$$
\Theta^{*}=\left\{\theta \in \mathbb{R}^{5} ; \mu \neq 0, \beta \neq 0, \sigma_{x}^{2} \geq 0, \sigma_{\delta}^{2} \geq 0, \sigma_{\epsilon}^{2} \geq 0, V(\theta)>0\right\} .
$$

Observe que é possível estabelecer um modelo estatístico (identificável) usando (3.5) como definição da distribuição da amostra e supondo que $\theta$ está no conjunto

$$
\Theta=\left\{\theta \in \mathbb{R}^{5} ; \mu \neq 0, \beta \neq 0, V(\theta)>0\right\}
$$

já que podemos ter $V(\theta)>0$ sem que necessariamente $\sigma_{x}^{2}, \sigma_{\delta}^{2}$, e $\sigma_{\epsilon}^{2}$ sejam todos não negativos (tome $\beta=1, \sigma_{x}^{2}=2, \sigma_{\delta}^{2}=-1$ e $\sigma_{\epsilon}^{2}=3$, por exemplo). A família de distribuições amostrais associada ao MPO está contida na família de distribuições amostrais associadas a este modelo estatístico.

É fácil mostrar que a função $f(\theta)=\left[(\mu, \beta \mu)^{\prime}, V(\theta)\right], \theta \in \Theta$ é uma reparametrização do modelo estatístico definido por uma amostra aleatória $\left\{\left(X_{i}, Y_{i}\right)^{\prime}, i \in \mathbb{N}\right\}$ proveniente de uma distribuição normal bivariada, com parâmetro $(\xi, \Sigma)$ no conjunto

$$
\Xi=\left\{\left(\xi_{1}, \xi_{2}\right)^{\prime} \in \mathbb{R}^{2} ; \xi_{1} \neq 0, \xi_{2} \neq 0\right\} \times \mathcal{M}_{2}
$$

Para um tamanho de amostra fixado $n$, temos que o estimador de máxima verossimilhança para $(\xi, \Sigma)$ é

$$
(\bar{\xi}, \bar{\Sigma})=\left[(\bar{X}, \bar{Y})^{\prime},\left(\begin{array}{cc}
S_{X} & S_{X Y} \\
S_{Y X} & S_{Y}
\end{array}\right)\right]
$$

(ver a definição em (1)). O estimador de máxima verossimilhança para $\theta$ no modelo reparametrizado é

$$
\bar{\theta}=\left(\bar{\mu}, \bar{\beta}, \bar{\sigma}_{x}^{2}, \bar{\sigma}_{\delta}^{2}, \bar{\sigma}_{\epsilon}^{2}\right)^{\prime}
$$

solução de $f(\bar{\theta})=(\bar{\xi}, \bar{\Sigma})$. Temos que

$$
\left(\bar{\mu}, \bar{\beta}, \bar{\sigma}_{x}^{2}, \bar{\sigma}_{\delta}^{2}, \bar{\sigma}_{\epsilon}^{2}\right)=\left(\bar{X}, \frac{\bar{Y}}{\bar{X}}, S_{X Y}\left(\frac{\bar{X}}{\bar{Y}}\right), S_{X}-S_{X Y}\left(\frac{\bar{X}}{\bar{Y}}\right), S_{Y}-S_{X Y}\left(\frac{\bar{Y}}{\bar{X}}\right)\right) \text {. }
$$

Seja $L$ a verossimilhança associada ao MPO. Se $\bar{\theta} \in \Theta^{*}$, ou seja, se $\bar{\sigma}_{x}^{2} \geq 0, \bar{\sigma}_{\delta}^{2} \geq 0$ e $\bar{\sigma}_{\epsilon}^{2} \geq 0$, então $\bar{\theta}$ maximiza $L$, pois $\Theta^{*} \subset \Theta$. Caso contrário, $L$ deve ser maximizada 
diretamente em $\Theta^{*}$. Aplicando métodos de maximização de funções com restrições de desigualdade como os descritos em Luenberger (1984, cap. 10), vemos que o problema reduz-se simplesmente à determinação de pontos críticos de $L$ restrita a cada um dos seguintes conjuntos: $A=\left\{\theta \in \Theta ; \sigma_{x}^{2}=0, \sigma_{\delta}^{2}>0, \sigma_{\epsilon}^{2}>0\right\}, B=\left\{\theta \in \Theta ; \sigma_{x}^{2}>\right.$ $\left.0, \sigma_{\delta}^{2}=0, \sigma_{\epsilon}^{2}>0\right\}$ e $C=\left\{\theta \in \Theta ; \sigma_{x}^{2}>0, \sigma_{\delta}^{2}>0, \sigma_{\epsilon}^{2}=0\right\}$. Estes pontos críticos são dados respectivamente por

$$
\begin{aligned}
& \tilde{\theta}_{A}=\left(\bar{X}, \frac{\bar{Y}}{\bar{X}}, 0, S_{X}, S_{Y}\right)^{\prime} \\
& \tilde{\theta}_{B}=\left(\bar{X}, \frac{d_{X Y}}{d_{X}}, S_{X}, 0, d_{Y}-\frac{\left(d_{X Y}\right)^{2}}{d_{X}}\right)^{\prime} \mathrm{e} \\
& \tilde{\theta}_{C}=\left(\bar{Y}\left(\frac{d_{X Y}}{d_{Y}}\right), \frac{d_{Y}}{d_{X Y}},\left(\frac{d_{X Y}}{d_{Y}}\right)^{2} S_{Y}, d_{X}-\frac{\left(d_{X Y}\right)^{2}}{d_{Y}}, 0\right)^{\prime},
\end{aligned}
$$

onde

$$
d_{X Y}=\frac{1}{n} \sum_{i=1}^{n} X_{i} Y_{i}, \quad d_{X}=\frac{1}{n} \sum_{i=1}^{n} X_{i}^{2} \text { e } d_{Y}=\frac{1}{n} \sum_{i=1}^{n} Y_{i}^{2} .
$$

$\mathrm{O}$ estimador de máxima verossimilhança para $\theta$ é aquele que entre $\tilde{\theta}_{A}, \tilde{\theta}_{B}$ e $\tilde{\theta}_{C}$ maximiza a função $L$. Estes resultados foram obtidos por Chan \& Mak (1979) e estão sumarizados no teorema 3.1.1.

Teorema 3.1.1 Seja $L$ a função de verossimilhança para o modelo passando pela origem baseada em $n$ observações. Sejam $\bar{\theta}=\left(\bar{\mu}, \bar{\beta}, \bar{\sigma}_{x}^{2}, \bar{\sigma}_{\delta}^{2}, \bar{\sigma}_{\epsilon}^{2}\right)$ dado em (3.9) e $\tilde{\theta}_{A}$, $\tilde{\theta}_{B}$ e $\tilde{\theta}_{C}$ dados em (3.10). O estimador de máxima verossimilhança para $\theta$ no modelo passando pela origem, $\tilde{\theta}=\left(\tilde{\mu}, \tilde{\beta}, \tilde{\sigma}^{2}, \tilde{\sigma}_{\delta}^{2}, \tilde{\sigma}_{\epsilon}^{2}\right)$, é dado por

$$
\tilde{\theta}=\bar{\theta} \text { se } \bar{\sigma}^{2} \geq 0, \bar{\sigma}_{\delta}^{2} \geq 0 \text { e } \bar{\sigma}_{\epsilon}^{2} \geq 0
$$

Caso contrário, é dado por $\tilde{\theta} \in\left\{\tilde{\theta}_{A}, \tilde{\theta}_{B}, \tilde{\theta}_{C}\right\}$ que satisfaz

$$
L(\tilde{\theta})=\max \left\{L\left(\tilde{\theta}_{A}\right), L\left(\tilde{\theta}_{B}\right), L\left(\tilde{\theta}_{C}\right)\right\} .
$$

Considere novamente o modelo

$$
\left\{\left(X_{i}, Y_{i}\right)^{\prime}, i \in \mathbb{N}\right\} \sim N I I D(\xi, \Sigma),(\xi, \Sigma) \in \Xi
$$


onde $\Xi$ está definido em (3.7), e o estimador de máxima verossimilhança em (3.8). Escreva $\Sigma=\left(\Sigma_{i j}\right)$. Temos que ${ }^{1}$

$$
\left\{n^{1 / 2}\left[\left(\bar{X}, \bar{Y}, S_{X}, S_{Y X}, S_{Y}\right)^{\prime}-\left(\xi^{\prime}, \Sigma_{11}, \Sigma_{21}, \Sigma_{22}\right)^{\prime}\right]\right\} \stackrel{\mathcal{D}(\xi, \Sigma)}{\longrightarrow} N\left(0, \mathcal{I}^{-1}(\xi, \Sigma)\right)
$$

onde $\mathcal{I}$ é a matriz de informação de Fisher. Este resultado é bastante conhecido e podemos encontrar a forma explícita da matriz de informação em, por exemplo, Lehmann (1983, exemplo 5.5, cap. 6). Concluimos então que

$$
n^{1 / 2}(\bar{\theta}-\theta) \stackrel{\mathcal{D}(\theta)}{\longrightarrow} N(0, \Sigma(\theta))
$$

para todo $\theta \in \Theta$, onde ${ }^{2}$

$$
\Sigma(\theta)=[G(f(\theta))]^{\prime} \mathcal{I}^{-1}(f(\theta)) G(f(\theta)),
$$

e $G(f(\theta))$ é a matriz Jacobiana de $f^{-1}$ avaliada em $f(\theta)$. Para os propósitos desta tese, não precisamos de todas as entradas da matriz $\Sigma(\theta)$, somente a parte referente à $\bar{\beta}$, que será obtida na seção 3.2. A forma completa da matriz pode ser encontrada em Chan \& Mak (1979, seção 3).

Se $\theta$ for tal que $\sigma_{x}^{2}>0, \sigma_{\delta}^{2}>0$ e $\sigma_{\epsilon}^{2}>0$ então o limite em distribuição de $\left\{n^{1 / 2}\left(\tilde{\theta}_{n}-\theta\right)\right\}$ é o mesmo de $\left\{n^{1 / 2}\left(\bar{\theta}_{n}-\theta\right)\right\}$. É o que diz o próximo resultado.

Teorema 3.1.2 Considere o modelo definido por (3.5), com $\theta \in \Theta$, e os estimadores $\bar{\theta}$ e $\tilde{\theta}$ dados em (3.9) e no teorema 3.1.1, respectivamente. Então, para todo $\theta$ tal que $\sigma_{x}^{2}>0, \sigma_{\delta}^{2}>0$ e $\sigma_{\epsilon}^{2}>0$,

1. $\lim _{n \rightarrow \infty} P_{\theta}(\tilde{\theta}=\bar{\theta})=1$;

2. $n^{1 / 2}(\tilde{\theta}-\theta) \stackrel{\mathcal{D}(\theta)}{\longrightarrow} N(0, \Sigma(\theta))$.

Demonstração: O conjunto

$$
A=\left\{\theta \in \Theta ; \sigma_{x}^{2}>0, \sigma_{\delta}^{2}>0, \sigma_{\epsilon}^{2}>0\right\}
$$

é aberto em $\mathbb{R}^{5}$. É fácil provar que $\{\bar{\theta}\}$ é fortemente consistente. Como consequência de $\bar{\theta} \rightarrow \theta \in A$ vem que $\bar{\theta} \in A$ para $n$ suficientemente grande. Assim,

$$
1=P_{\theta}[\bar{\theta} \rightarrow \theta] \leq P_{\theta}[\liminf (\bar{\theta} \in A)] \leq \liminf P_{\theta}[\bar{\theta} \in A] \leq 1,
$$

\footnotetext{
${ }^{1}$ A rigor, deveríamos escrever $\bar{X}_{n},\left(S_{X}\right)_{n}$, etc. mas preferimos omitir o subscrito para tornar a notação um pouco mais leve.

${ }^{2}$ Aqui nos permitimos um abuso de notação, pois o mais correto seria redefinir a função $f$ como $f(\theta)=\left(\mu, \beta \mu, \sigma_{x}^{2}+\sigma_{\delta}^{2}, \beta \sigma_{x}^{2}, \beta^{2} \sigma_{x}^{2}+\sigma_{\epsilon}^{2}\right)$.
} 
e, portanto, $P_{\theta}(\bar{\theta} \in A) \rightarrow 1$ quando $n \rightarrow \infty$. Mas $(\bar{\theta} \in A) \subset(\bar{\theta}=\tilde{\theta})$, demonstrando a primeira parte. A segunda parte demonstra-se observando que $n^{1 / 2}(\bar{\theta}-\tilde{\theta}) \stackrel{\operatorname{Pr}(\theta)}{\longrightarrow} 0 \mathrm{e}$ que

$$
n^{1 / 2}(\tilde{\theta}-\theta)=n^{1 / 2}(\bar{\theta}-\theta)+n^{1 / 2}(\tilde{\theta}-\bar{\theta})
$$

O resultado segue então pelo Teorema de Slutsky.

Ou seja, $\{\tilde{\theta}\}$ é uma sequência de estimadores assintoticamente normal e eficiente ${ }^{3}$, se restringirmos o espaço paramétrico do MPO considerando somente os pontos com $\sigma_{x}^{2}>0, \sigma_{\delta}^{2}>0$ e $\sigma_{\epsilon}^{2}>0$.

\subsubsection{Modelo com a Razão das Variâncias dos Erros de Ob- servação Conhecida}

Uma outra alternativa para tornar o modelo em (3.4) identificável é considerar que a razão

$$
\lambda=\frac{\sigma_{\epsilon}^{2}}{\sigma_{\delta}^{2}}
$$

é conhecida. O vetor de parâmetros neste caso é $\theta=\left(\mu, \alpha, \beta, \sigma_{x}^{2}, \sigma_{\delta}^{2}\right)^{\prime}$ e o espaço paramétrico é o conjunto

$$
\Theta=\left\{\theta \in \mathbb{R}^{5} ; \sigma_{x}^{2} \geq 0, \sigma_{\delta}^{2} \geq 0, V(\theta)>0\right\}
$$

onde

$$
V(\theta)=\left(\begin{array}{cc}
\sigma_{x}^{2}+\sigma_{\delta}^{2} & \beta \sigma_{x}^{2} \\
\beta \sigma_{x}^{2} & \beta^{2} \sigma_{x}^{2}+\lambda \sigma_{\delta}^{2}
\end{array}\right) .
$$

Seja $f(\theta)=\left[(\mu, \alpha+\beta \mu)^{\prime}, V(\theta)\right], \theta \in \Theta$. Seja $(\xi, \Sigma) \in \mathbb{R}^{2} \times \mathcal{M}_{2}, \Sigma=\left(\Sigma_{i j}\right)$. É sempre possível encontrar $\theta \in \Theta$ tal que $f(\theta)=(\xi, \Sigma)$. Se $\Sigma_{12} \neq 0$, então existe um único $\theta \in \Theta$ tal que $f(\theta)=(\xi, \Sigma)$. Assim, quando $S_{X Y} \neq 0$, a equação $f(\hat{\theta})=(\bar{\xi}, \bar{\Sigma})$ (ver 3.8) possui uma única solução, exibida no teorema 3.1.3 (ver, por exemplo, Fuller, 1987, seção 1.3).

\footnotetext{
${ }^{3}$ No sentido de que a sua matriz de covariâncias assintótica é igual a $n^{-1}$ vezes a matriz de informação de Fisher.
} 
Teorema 3.1.3 O estimador de máxima verossilhança para $\theta$ no modelo com a razão das variâncias dos erros de observação conhecida, $\hat{\theta}=\left(\hat{\mu}, \hat{\alpha}, \hat{\beta}, \hat{\sigma_{x}^{2}}, \hat{\sigma_{\delta}^{2}}\right)$, é dado por

$$
\begin{aligned}
\hat{\mu} & =\bar{X} \\
\hat{\alpha} & =\bar{Y}-\hat{\beta} \bar{X} \\
\hat{\beta} & =\frac{S_{Y}-\lambda S_{X}+\left[\left(S_{Y}-\lambda S_{X}\right)^{2}+4 \lambda S_{X Y}^{2}\right]^{1 / 2}}{2 S_{X Y}} \\
\hat{\sigma_{x}^{2}} & =(2 \lambda)^{-1}\left\{\left[\left(S_{Y}-\lambda S_{X}\right)^{2}+4 \lambda S_{X Y}^{2}\right]^{1 / 2}-\left(S_{Y}-\lambda S_{X}\right)\right\} \\
\hat{\sigma_{\delta}^{2}} & =(2 \lambda)^{-1}\left\{S_{Y}+\lambda S_{X}-\left[\left(S_{Y}-\lambda S_{X}\right)^{2}+4 \lambda S_{X Y}^{2}\right]^{1 / 2}\right\} .
\end{aligned}
$$

Observe que $S_{X Y}$ é diferente de zero com probabilidade 1 , de modo que $\hat{\theta}$ maximiza a verossimilhança associada ao modelo com probabilidade 1 , e assim é estimador de máxima verossimilhança para $\theta$. Se tivermos $\lambda>0$ e $\theta$ for tal que $\sigma^{2}>0, \sigma_{\delta}^{2}>0$, então $\theta$ está no interior de $\Theta$ e

$$
n^{1 / 2}(\hat{\theta}-\theta) \stackrel{\mathcal{D}(\theta)}{\longrightarrow} N(0, \Sigma(\theta))
$$

onde $\Sigma(\theta)$ é como em (3.12) (se $\lambda=0$ o determinante de $V(\theta)$ pode ser nulo e, conseqüentemente, $\theta \notin \Theta)$. Pelo Teorema 1.3.1 de Fuller $(1987),\left\{n^{1 / 2}\left[\left(\hat{\alpha}, \hat{\beta}, \hat{\sigma_{x}^{2}}\right)^{\prime}-\right.\right.$ $\left.\left.\left(\alpha, \beta, \sigma_{\delta}^{2}\right)\right]\right\}$ converge em distribuição para uma normal com média zero e matriz de covariâncias

$$
\left[\begin{array}{ccc}
a+\mu^{2} \Gamma & -\mu \Gamma & 0 \\
-\mu \Gamma & \Gamma & 0 \\
0 & 0 & 2 \sigma_{\delta}^{4}
\end{array}\right],
$$

onde $\Gamma=\sigma^{-4}\left(a \sigma_{x}^{2}+a \sigma_{\delta}^{2}-b^{2}\right), a=\sigma_{\epsilon}^{2}+\beta^{2} \sigma_{\delta}^{2}, b=-\beta \sigma_{\delta}^{2}$. Portanto,

$$
n^{1 / 2}(\hat{\beta}-\beta) \stackrel{\mathcal{D}(\theta)}{\longrightarrow} N(0, \Gamma),
$$

quando $\sigma_{x}^{2}>0, \sigma_{\delta}^{2}>0$ e $\lambda>0$, um resultado que utilizaremos em seguida.

\subsection{Testes de Distância para Algumas Hipóteses Restritas}

\subsubsection{Modelo Passando Pela Origem}

Considere o MPO com espaço paramétrico $\Theta^{*}$ restrito aos pontos $\theta$ tais que $\sigma_{x}^{2}>0, \sigma_{\delta}^{2}>0$ e $\sigma_{\epsilon}^{2}>0$. O objetivo desta seção é a formulação de um teste de 
distância para as hipóteses:

$$
H: \beta=\beta_{0} \text { contra } K: \beta>\beta_{0}
$$

com $\beta_{0}$ conhecido. Isto pode ser aplicado, por exemplo, em experimentos pré-teste, pós-teste, onde parece ser mais natural considerar que a inclinação $\beta$ é maior ou igual que $\beta_{0}=1$. Por exemplo: espera-se que o desempenho de estudantes em um teste aplicado após um período de treino seja melhor que o desempenho em um teste aplicado ao início do curso. Neste caso, os valores observados $\left\{\left(X_{i}, Y_{i}\right)^{\prime}, i=1, \ldots, n\right\}$ são os graus obtidos ao início $\left(X_{i}\right)$ e ao final do curso $\left(Y_{i}\right)$. Podemos ver $\left(X_{i}, Y_{i}\right)^{\prime}$ como uma "estimativa" do verdadeiro conhecimento em torno dos tópicos cobertos pelo curso.

Pelo Teorema 3.1.2,

$$
n^{1 / 2}(\tilde{\theta}-\theta) \stackrel{\mathcal{D}(\theta)}{\longrightarrow} N(0, \Sigma(\theta)),
$$

para todo $\theta \in \Theta^{*}$, onde $\tilde{\theta}$ é descrito no Teorema 3.1.1. Assim,

$$
n^{1 / 2}(\tilde{\beta}-\beta) \stackrel{\mathcal{D}(\theta)}{\longrightarrow} N\left(0, \Sigma_{\beta}(\theta)\right),
$$

para todo $\theta \in \Theta^{*}$, onde $\Sigma_{\beta}(\theta)$ é o elemento $(2,2)$ da matriz $\Sigma(\theta)$. Pelo Teorema Central do Limite, temos que

$$
n^{1 / 2}\left[(\bar{X}, \bar{Y})^{\prime}-(\mu, \beta \mu)^{\prime}\right] \stackrel{\mathcal{D}(\theta)}{\longrightarrow} N[0, V(\theta)]
$$

e, conseqüentemente,

$$
n^{1 / 2}\left(\frac{\bar{Y}}{\bar{X}}-\beta\right) \stackrel{\mathcal{D}(\theta)}{\longrightarrow} N\left[0, \mu^{-2}(-\beta, 1) V(\theta)(-\beta, 1)^{\prime}\right],
$$

para todo $\theta \in \Theta$. Como $\bar{\beta}=\bar{Y} / \bar{X}$, temos que

$$
\Sigma_{\beta}(\theta)=\frac{\beta^{2} \sigma_{\delta}^{2}+\sigma_{\epsilon}^{2}}{\mu^{2}} .
$$

Assim, uma sequência de estimadores consistente para $\Sigma_{\beta}(\theta)$ é dada por

$$
\tilde{\Sigma}_{\beta}=\Sigma_{\beta}(\tilde{\theta}) .
$$

A estatística para o teste de distância é dada por

$$
\mathbf{D}=n \frac{\left(\tilde{\beta}-\beta_{0}\right)^{2}}{\tilde{\Sigma}_{\beta}} I_{\left[\beta_{0}, \infty\right)}(\tilde{\beta}) .
$$


Sob $H$ temos que a distribuição assintótica de $\mathbf{D}$ é

$$
F(c)=\left(\frac{1}{2}+\frac{1}{2} P\left(\chi_{1}^{2} \leq c\right)\right) I_{[0, \infty)}(c) .
$$

$\mathrm{O}$ teste de distância rejeita $H$ ao nível assintótico $\xi$ quando $\mathbf{D}>c_{\xi}$, onde $c_{\xi}$ é tal que $P\left(\chi_{1}^{2} \geq c_{\xi}\right)=2 \xi$.

Neste ponto é instrutivo comparar o teste de distância com um teste "naive" para as hipóteses $H$ e $K$. Entendemos por teste "naive" como sendo uma regra de decisão formulada sem levar em consideração a hipótese de que a variável regressora é observada com erro. Sob este ponto de vista, um modelo aparentemente adequado para análise de dados tipo pré-teste, pós-teste é o modelo de regressão linear simples passando pela origem tradicional, definido por

$$
Y_{i}=\beta X_{i}+\epsilon_{i}, i=1, \ldots, n,
$$

onde $\left\{\epsilon_{i}, i=1, \ldots, n\right\} \sim N I I D\left(0, \tau^{2}\right), \tau^{2}>0$, são erros de mensuração e $X=$ $\left(X_{1}, \ldots, X_{n}\right)^{\prime}$ é um vetor de constantes com $\|X\|^{2}>0$. Seja $Y=\left(Y_{1}, \ldots, Y_{n}\right)^{\prime} \mathrm{e}$ seja $\hat{\beta}$ o estimador de mínimos quadrados para $\beta$, definido por

$$
\|Y-\hat{\beta} X\|^{2}=\min \left\{\|Y-\beta X\|^{2} ; \beta \in \mathbb{R}\right\} .
$$

Então $\hat{\beta}=X^{\prime} Y /\|X\|^{2}$ e, conseqüentemente, $\hat{\beta} \sim N\left(\beta, \tau^{2}\|X\|^{-2}\right)$. Seja $\tilde{\beta}$ o estimador de mínimos quadrados restrito, definido por

$$
\|Y-\tilde{\beta} X\|^{2}=\min \left\{\|Y-\beta X\|^{2} ; \beta \geq \beta_{0}\right\}
$$

Então

$$
\tilde{\beta}=\left\{\begin{array}{lll}
\hat{\beta} & \text { se } & \hat{\beta} \geq \beta_{0} \\
\beta_{0} & \text { se } & \hat{\beta}<\beta_{0}
\end{array} .\right.
$$

Uma estatística "tipo Wald" para testar $H$ contra $K$ é dada por

$$
\mathbf{Q}=\frac{\left(\tilde{\beta}-\beta_{0}\right)^{2}}{S^{2}\|X\|^{-2}}
$$

onde $(n-1) S^{2}=\|Y-\hat{\beta} X\|^{2}$. Assim,

$$
\mathrm{Q}=\frac{\left(\hat{\beta}-\beta_{0}\right)^{2}}{S^{2}\|X\|^{-2}} I_{\left[\beta_{0}, \infty\right)}(\hat{\beta}) .
$$


Temos que, sob $H$,

$$
\left(\hat{\beta}-\beta_{0}\right)^{2} / \tau^{2}\|X\|^{-2} \sim \chi_{1}^{2} .
$$

Além disso, $(n-1) S^{2} / \tau^{2} \sim \chi_{n-1}^{2}$ e $\hat{\beta}$ e $S^{2}$ são independentes. Assim, sob $H$,

$$
\frac{\left(\hat{\beta}-\beta_{0}\right)^{2}}{S^{2}\|X\|^{-2}} \sim t_{n-1}^{2},
$$

onde $t_{n-1}$ denota uma variável aleatória com distribuição $t$ de Student com $n-1$ graus de liberdade. Portanto, a distribuição nula de $\mathbf{Q}$ é dada por

$$
P(\mathrm{Q} \leq c)=\left(\frac{1}{2}+\frac{1}{2} P\left(t_{n-1}^{2} \leq c\right)\right) I_{[0, \infty)}(c)
$$

Conseqüentemente, a região crítica de nível $\xi$ é dada por $\left(\mathrm{Q}>c_{\xi}\right)$, onde $c_{\xi}$ é tal que $P\left(t_{n-1} \geq c_{\xi}^{1 / 2}\right)=\xi$. Este teste foi sugerido por Paula \& Bolfarine (1997).

Efetuamos, através de dados simulados, um estudo de poder. Amostras do MPO $\operatorname{com} \beta \geq 1$ foram geradas e os testes de distância e "naive" para $H: \beta=1$ contra $K: \beta>1$ foram aplicados. Fizemos uma pequena modificação na expressão da estatística de distância, substituindo $\tilde{\beta}$ por 1 (o valor conhecido de $\beta$ sob $H$ ) em $\tilde{\Sigma}_{\beta}$. Tomamos sempre $\mu=\sigma_{x}^{2}=\sigma_{\epsilon}^{2}=1$. Para cada combinação de $n, \sigma_{\delta}^{2}$ e $\beta, 1000$ amostras foram geradas. A tabela 3.1 apresenta as taxas de rejeição para $n=20$. Em parênteses temos os resultados para o teste "naive". Os níveis nominais são de $5 \%$ e $10 \%$. Na presença de erro na observação da variável explanadora, temos desempenho muito superior do teste de distância. A quarta coluna nesta tabela mostra os níveis empíricos para os dois testes. Note que o nível do teste "naive" é fortemente afetado por erros de observação: quando $\sigma_{\delta}^{2}$ cresce este teste mostrase extremamente conservador. Sob $H$, a aproximação da função de distribuição de $\mathrm{D}$ a $F$ é muito boa, mesmo para um tamanho de amostra moderado. Por exemplo, considere $n=20$, todos os parâmetros iguais a 1 e as hipóteses $H^{*}: P(\mathbf{D} \leq c)=F(c)$ para todo $c, H^{* *}: P(\mathbf{D} \leq c) \neq F(c)$ para algum $c$. Para testar a qualidade do ajuste, aplicamos um teste de Kolmogorov para distribuições descontínuas (Conover, 1980) para testar $H^{*}$ contra $H^{* *}$. Com 30 observações da estatística de teste, obtivemos uma probabilidade de significância do teste que está entre 0.376 e 0.420 , levando à aceitação de $H^{*}$. 
Tabela 3.1: Taxas de Rejeição para os Testes de Distância (e "naive") $(H: \beta=1$, $K: \beta>1)$.

\begin{tabular}{ccccccccc}
\hline$n$ & $\sigma_{\delta}^{2}$ & nível & \multicolumn{6}{c}{$\beta$} \\
& & & 1.0 & 1.05 & 1.10 & 1.15 & 1.20 & 1.25 \\
\hline 20 & 0.5 & $5 \%$ & 0.065 & 0.098 & 0.139 & 0.209 & 0.260 & 0.319 \\
& & & $(0.008)$ & $(0.017)$ & $(0.035)$ & $(0.049)$ & $(0.112)$ & $(0.151)$ \\
& & $10 \%$ & 0.116 & 0.160 & 0.231 & 0.290 & 0.374 & 0.428 \\
& & $(0.022)$ & $(0.043)$ & $(0.088)$ & $(0.105)$ & $(0.196)$ & $(0.253)$ \\
\hline & & $5 \%$ & 0.056 & 0.078 & 0.098 & 0.132 & 0.199 & 0.227 \\
& & $(0.000)$ & $(0.000)$ & $(0.001)$ & $(0.003)$ & $(0.005)$ & $(0.005)$ \\
& \multirow{2}{*}{$10 \%$} & 0.107 & 0.135 & 0.161 & 0.237 & 0.279 & 0.339 \\
& & $(0.001)$ & $(0.000)$ & $(0.004)$ & $(0.009)$ & $(0.014)$ & $(0.010)$ \\
\hline & 1.5 & $5 \%$ & 0.045 & 0.056 & 0.099 & 0.110 & 0.119 & 0.150 \\
& & $(0.000)$ & $(0.000)$ & $(0.000)$ & $(0.000)$ & $(0.000)$ & $(0.000)$ \\
& \multirow{2}{*}{$10 \%$} & 0.091 & 0.113 & 0.175 & 0.179 & 0.197 & 0.251 \\
& & $(0.000)$ & $(0.000)$ & $(0.000)$ & $(0.000)$ & $(0.000)$ & $(0.000)$ \\
\hline
\end{tabular}

\subsubsection{Uma Aplicação}

A tabela 3.2, retirada de Moore \& McCabe (1993), apresenta escores pré-teste, pós-teste em um curso intensivo de língua espanhola para 20 professores do segundo grau. Espera-se que o curso melhore a habilidade auditiva dos estudantes. Neste caso, consideramos que $x_{i}, i=1, \ldots, n$, os reais escores dos estudantes no pré-teste, são variáveis aleatórias que não podem ser observadas diretamente e que os escores observados são uma mera "estimativa" destes. Com efeito, o escore observado pode subestimar ou superestimar o verdadeiro conhecimento. Superestimação ocorre, por exemplo, quando o estudante "chuta" corretamente algumas das questões. Um caso de subestimação pode ocorrer se o estudante fica tenso ou perde a concentração na hora de responder as questões. Podemos argumentar de maneira similar para os escores do pós-teste, de tal maneira que o escore observado é só uma estimativa do verdadeiro conhecimento $\beta x_{i}$. Assim, neste caso, a inclinação $\beta$ relaciona o verdadeiro conhecimento dos estudantes e não somente os escores observados, os quais podem apresentar vícios. Por estas razões acreditamos que, considerando erros nas observações, temos uma comparação mais direta baseada na real melhora do estu- 
dante e não somente nos escores observados. Portanto, assumindo o MPO podemos verificar se o curso melhora a habilidade auditiva testando as hipóteses $H: \beta=1$ contra $K: \beta>1$.

A estimativa de máxima verossimilhança é $\tilde{\beta}=1.053$ com estimativa de desviopadrão assintótico 0.265 . O valor da estatística do teste de distância é $\mathbf{D}=4.335$, o qual leva a uma probabilidade de significância do teste igual a 0.019. Se o teste "naive" fosse aplicado a probabilidade de significância seria 0.060 , o que leva à aceitação de $H$ caso usemos o nível $5 \%$.

Tabela 3.2: Escores Pré-teste e Pós-teste em um Teste de Audição em Língua Espanhola

\begin{tabular}{cccccc}
\hline Indivíduo & Pré-teste & Pós-teste & Indivíduo & Pré-teste & Pós-teste \\
\hline 1 & 30 & 29 & 11 & 30 & 32 \\
2 & 28 & 30 & 12 & 29 & 28 \\
3 & 31 & 32 & 13 & 31 & 34 \\
4 & 26 & 30 & 14 & 29 & 32 \\
5 & 20 & 16 & 15 & 34 & 32 \\
6 & 30 & 25 & 16 & 20 & 27 \\
7 & 34 & 31 & 17 & 26 & 28 \\
8 & 15 & 18 & 18 & 25 & 29 \\
9 & 28 & 33 & 19 & 31 & 32 \\
10 & 20 & 25 & 20 & 29 & 32 \\
\hline
\end{tabular}

\subsubsection{Modelo com Razão de Variâncias Conhecida}

Considere agora o modelo definido na seção 3.1.3. Para testar $H: \beta=\beta_{0}$ contra $K: \beta>\beta_{0}$ a estatística do teste de distância é definida por

$$
\mathbf{D}=n \frac{\left(\hat{\beta}-\beta_{0}\right)^{2}}{\hat{\Sigma}_{\beta}} I_{\left[\beta_{0}, \infty\right)}(\hat{\beta}),
$$

onde

$$
\hat{\Sigma}_{\beta}=\frac{\hat{\sigma_{\delta}^{2}}\left[{\hat{\sigma_{x}^{2}}}^{2}\left(\hat{\beta}^{2}+\lambda\right)+\lambda \hat{\sigma_{\delta}^{2}}\right]}{\hat{\sigma_{x}^{4}}} .
$$


A distribuição nula limite de $\mathbf{D}$ também é $F$, dada em (3.16), de tal maneira que rejeitamos $H$ ao nível assintótico $\xi$ quando $\mathbf{D}>c_{\xi}$, onde $c_{\xi}$ é tal que $\operatorname{Pr}\left(\chi_{1}^{2} \geq c_{\xi}\right)=$ $2 \xi$.

Fuller (1987) apresenta um teste exato para $H$ contra a alternativa $\beta \neq \beta_{0}$, $\beta \geq 0$. Através de dados simulados, tentaremos obter uma idéia do comportamento das funções poder desse teste e do teste de distância em hipóteses com $\beta \geq \beta_{0}$. A definição do teste exato baseia-se no teorema abaixo.

Teorema 3.2.1 Considere o modelo com razão de variâncias conhecida e suponha que $\beta \geq 0$. Então $\beta=\beta_{0}$ se e somente se $Y_{i}-\beta_{0} X_{i}$ e $(1 / \lambda) \beta_{0} Y_{i}+X_{i}$ são variáveis aleatórias independentes, $i=1, \ldots, n$.

Demonstração: Temos que

$$
\operatorname{Cov}\left(Y_{i}-\beta_{0} X_{i}, \frac{1}{\lambda} \beta_{0} Y_{i}+X_{i}\right)=\frac{1}{\lambda} \beta_{0} \sigma_{x}^{2} \beta^{2}+\left(\sigma_{x}^{2}-\frac{1}{\lambda} \beta_{0}^{2} \sigma_{x}^{2}\right) \beta-\beta_{0} \sigma_{x}^{2} .
$$

Os valores de $\beta$ que tornam essa covariância nula são as raízes do polinômio em $\beta$ no lado direito da igualdade acima. Estas raízes são $\beta_{0} \mathrm{e}-\lambda / \beta_{0}$. Como sabemos que $\beta \geq 0$, temos o resultado.

Assim, um teste para $\beta=\beta_{0}$ contra $\beta \neq \beta_{0}, \beta \geq 0$ é o teste usual para $\rho=0$ contra $\rho \neq 0$, onde $\rho$ é o coeficiente de correlação entre as duas variáveis definidas no enunciado do Teorema.

Um estudo de poder nos mesmos padrões estabelecidos para o MPO foi realizado. Na Tabela 3.3 apresentamos alguns resultados para um tamanho de amostra $n=20$ e $\alpha=0.5, \mu=1, \lambda=1, \sigma_{x}^{2}=1$. Em parênteses temos as taxas para o teste de Fuller.

\subsection{Extensões para Duas ou Mais Retas}

Nesta seção fazemos uma extensão da teoria apresentada na seção 3.2 , formulando testes de distância para hipóteses restritas envolvendo as inclinações em $k$ amostras independentes do MPO. A teoria é válida também para o modelo com razão de variâncias conhecida, com óbvias adaptações.

Sejam

$$
\left\{\left(X_{i j}, Y_{i j}\right)^{\prime}, j \in \mathbb{N}\right\} \sim N I I D\left[\left(\mu_{i}, \beta_{i} \mu_{i}\right)^{\prime}, V\left(\theta_{i}\right)\right], i=1, \ldots, k
$$


Tabela 3.3: Taxas de Rejeição para os Testes de Distância (e de Fuller) $(H: \beta=1$, $K: \beta>1)$.

\begin{tabular}{ccccccccc}
\hline$n$ & $\sigma_{\delta}^{2}$ & nível & \multicolumn{6}{c}{$\beta$} \\
& & & 1.0 & 1.05 & 1.10 & 1.15 & 1.20 & 1.25 \\
\hline 20 & 0.5 & $5 \%$ & 0.063 & 0.102 & 0.175 & 0.262 & 0.342 & 0.477 \\
& & & $(0.048)$ & $(0.050)$ & $(0.090)$ & $(0.129)$ & $(0.203)$ & $(0.296)$ \\
& & \multirow{2}{*}{$10 \%$} & 0.114 & 0.167 & 0.265 & 0.379 & 0.482 & 0.604 \\
& & $(0.102)$ & $(0.108)$ & $(0.167)$ & $(0.216)$ & $(0.299)$ & $(0.414)$ \\
\hline \multirow{2}{*}{1.0} & $5 \%$ & 0.049 & 0.05 & 0.078 & 0.107 & 0.137 & 0.145 \\
& & $(0.049)$ & $(0.046)$ & $(0.063)$ & $(0.065)$ & $(0.089)$ & $(0.095)$ \\
& \multirow{2}{*}{$10 \%$} & 0.097 & 0.097 & 0.138 & 0.185 & 0.231 & 0.233 \\
& & $(0.101)$ & $(0.095)$ & $(0.116)$ & $(0.134)$ & $(0.161)$ & $(0.168)$ \\
\hline
\end{tabular}

$k$ amostras independentes do MPO, onde $\theta_{i}=\left(\mu_{i}, \beta_{i}, \sigma_{x i}^{2}, \sigma_{\delta i}^{2}, \sigma_{\epsilon i}^{2}\right)^{\prime}$. O vetor de parâmetros asociado ao modelo estatístico definido pelas amostras é $\theta=\left(\theta_{1}^{\prime}, \ldots, \theta_{k}^{\prime}\right)^{\prime}$. A distribuição amostral associada é $P_{\theta}=\prod_{i=1}^{k} P_{\theta_{i}}$. O espaço paramétrico é $\prod_{i=1}^{k} \Theta^{*}$, onde $\Theta^{*}$ é dado em (3.6), mas supondo que $\sigma_{x i}^{2}, \sigma_{\delta i}^{2}$ e $\sigma_{\epsilon i}^{2}$ são positivos, $i=1, \ldots, k$.

Primeiramente vamos estender o Teorema 3.1.2. Seja $n_{i_{1}}<n_{i_{2}}<\ldots$ uma sequência de tamanhos amostrais associados à amostra $i, i=1, \ldots, k$. Seja $N_{j}=$ $\sum_{i=1}^{k} n_{i_{j}}$ o tamanho amostral total. Vamos supor que

$$
\lim _{j \rightarrow \infty} \frac{n_{i_{j}}}{N_{j}}=\gamma_{i}, i=1, \ldots, k
$$

onde $\sum_{i=1}^{k} \gamma_{i}=1$ e $\gamma_{i}>0, i=1, \ldots, k$. Observe que, quando os tamanhos amostrais são iguais, ou seja, quando $n_{i j}=j$, temos $\gamma_{i}=1 / k$. Defina

$$
\Omega(\theta)=\text { block } \operatorname{diag}\left\{\gamma_{1}^{-1} \Sigma\left(\theta_{1}\right), \ldots, \gamma_{k}^{-1} \Sigma\left(\theta_{k}\right)\right\},
$$

onde $\Sigma\left(\theta_{i}\right)$ é definida em (3.12).

Teorema 3.3.1 Seja $\tilde{\theta}=\left(\tilde{\theta}_{1}^{\prime}, \ldots, \tilde{\theta}_{k}^{\prime}\right)^{\prime}$, onde $\tilde{\theta}_{i}$ é o estimador de máxima verossimilhança para $\theta_{i}$, definido no Teorema 3.1.1. Então

$$
N_{j}^{1 / 2}(\tilde{\theta}-\theta) \stackrel{\mathcal{D}(\theta)}{\longrightarrow} N[0, \Omega(\theta)], \text { quando } j \rightarrow \infty,
$$

para todo $\theta \in \prod_{i=1}^{k} \Theta^{*}$. 
Demonstração: Seja $\bar{\theta}=\left(\bar{\theta}_{1}^{\prime}, \ldots, \bar{\theta}_{k}^{\prime}\right)^{\prime}$, onde $\bar{\theta}_{i}$ é o estimador em (3.9) definido em cada amostra. Então

$$
P_{\theta}(\tilde{\theta}=\bar{\theta})=\prod_{i=1}^{k} P_{\theta_{i}}\left(\tilde{\theta}_{i}=\bar{\theta}_{i}\right)
$$

Pelo Teorema 3.1.2 vem que

$$
\lim _{j \rightarrow \infty} P_{\theta}(\tilde{\theta}=\bar{\theta})=1
$$

Como as amostras são independentes, temos, por (3.11), que

$$
\left[n_{1_{j}}^{1 / 2}\left(\bar{\theta}_{1}-\theta_{1}\right)^{\prime}, \ldots, n_{k_{j}}^{1 / 2}\left(\bar{\theta}_{k}-\theta_{k}\right)^{\prime}\right]^{\prime} \stackrel{\mathcal{D}(\theta)}{\longrightarrow} N\left(0, \text { block } \operatorname{diag}\left\{\Sigma\left(\theta_{1}\right), \ldots, \Sigma\left(\theta_{k}\right)\right\}\right)
$$

quando $j \rightarrow \infty$. Multiplicando o lado esquerdo da expressão acima por

$$
\operatorname{diag}\left\{\left(N_{j} / n_{1_{j}}\right)^{1 / 2}, \ldots,\left(N_{j} / n_{k_{j}}\right)^{1 / 2}\right\}
$$

fazendo $j$ tender para infinito e usando $(3.20)$ e (3.21), temos o resultado.

Sejam $\beta=\left(\beta_{1}, \ldots, \beta_{k}\right)^{\prime}$ o vetor de inclinações e $\tilde{\beta}=\left(\tilde{\beta}_{1}, \ldots, \tilde{\beta}_{k}\right)^{\prime}$ o seu estimador extraído de $\tilde{\theta}$. Como consequência imediata do Teorema 3.3.1 temos que

$$
N_{j}^{1 / 2}(\tilde{\beta}-\beta) \stackrel{\mathcal{D}(\theta)}{\longrightarrow} N\left[0, \Omega_{\beta}(\theta)\right], \text { quando } j \rightarrow \infty
$$

para todo $\theta \in \prod_{i=1}^{k} \Theta^{*}$, onde

$$
\Omega_{\beta}(\theta)=\operatorname{diag}\left\{\gamma_{1}^{-1}\left(\frac{\beta_{1}^{2} \sigma_{\delta 1}^{2}+\sigma_{\epsilon 1}^{2}}{\mu_{1}^{2}}\right), \ldots, \gamma_{k}^{-1}\left(\frac{\beta_{k}^{2} \sigma_{\delta k}^{2}+\sigma_{\epsilon k}^{2}}{\mu_{k}^{2}}\right)\right\}
$$

(ver 3.14 e 3.15 ).

Considere as hipóteses:

$$
H: \beta=\beta_{0}, \quad K: \beta \geq \beta_{0},
$$

onde $\beta_{0}=\left(\beta_{01}, \ldots \beta_{0 k}\right)^{\prime}$ é conhecido. Para o cálculo da estatística do teste de distância para $H$ contra $K$, precisamos obter a projeção (segundo a norma induzida pela matriz $\left.\Omega_{\beta}(\tilde{\theta})\right)$ de $N_{j}^{1 / 2}\left(\tilde{\beta}-\beta_{0}\right)$ sobre $\mathcal{O}_{k}$. Esta tarefa é simples, pois $\Omega_{\beta}(\tilde{\theta})$ é diagonal (veja o apêndice A). Temos que a $i$-ésima coordenada desta projeção é dada por

$$
N_{j}^{1 / 2}\left(\tilde{\beta}_{i}-\beta_{0 i}\right) I_{[0, \infty)}\left(\tilde{\beta}_{i}-\beta_{0 i}\right)
$$


resultando na seguinte expressão para a estatística de distância

$$
\mathbf{D}=\sum_{i=1}^{k} N_{j} \frac{\gamma_{i} \tilde{\mu}_{i}^{2}\left(\tilde{\beta}_{i}-\beta_{0 i}\right)^{2}}{\tilde{\beta}_{i}^{2} \tilde{\sigma}_{\delta i}^{2}+\tilde{\sigma}_{\epsilon i}^{2}} I_{[0, \infty)}\left(\tilde{\beta}_{i}-\beta_{0 i}\right) .
$$

Pelo Teorema 2.2.2 e pelas expressões para os pesos dadas no apêndice B temos, para $\theta \in H$,

$$
\begin{aligned}
\lim _{j \rightarrow \infty} P_{\theta}(\mathbf{D} \geq c) & =\sum_{i=0}^{k} P\left(\chi_{i}^{2} \geq c\right) w\left(k, i, \Omega_{\beta}(\theta)\right) \\
& =\sum_{i=0}^{k} P\left(\chi_{i}^{2} \geq c\right)\left(\begin{array}{c}
k \\
i
\end{array}\right) \frac{1}{2^{k}} .
\end{aligned}
$$

Assim, o teste de distância para $H$ contra $K$ de nível assintótico $\xi$ é definido por: rejeitar $H$ se e somente se $\mathbf{D}>c_{\xi}$, onde

$$
\sum_{i=0}^{k} P\left(\chi_{i}^{2} \geq c_{\xi}\right)\left(\begin{array}{c}
k \\
i
\end{array}\right) \frac{1}{2^{k}}=\xi .
$$

Na tabela 3.4 apresentamos um estudo de poder através de dados gerados artificialmente. Consideramos $k=3$ e utilizamos os testes de distância e Wald para testar

$$
H_{1}: \beta=(1,1,1)^{\prime} \text { contra } K_{1}: \beta \geq(1,1,1)^{\prime} .
$$

Em uma extensão da situação apresentada na seção 3.2 .2 , a hipótese $K_{1}$ significa que, de três turmas submetidas independentemente aos exames pré-teste e pós-teste, pelo menos uma apresenta melhora no nível de conhecimento.

Tomamos $n_{1}=n_{2}=n_{3}=n, \sigma_{x 1}^{2}=\sigma_{x 2}^{2}=\sigma_{x 3}^{2}=0.1, \sigma_{\delta 1}^{2}=\sigma_{\delta 3}^{2}=0.01, \sigma_{\delta 2}^{2}=0.05$, $\sigma_{\epsilon 1}^{2}=\sigma_{\epsilon 2}^{2}=\sigma_{\epsilon 3}^{2}=0.50, \mu_{1}=\mu_{2}=\mu_{3}=1, \beta_{1}=1$. Para cada combinação de parâmetros, 2000 amostras de tamanho $3 n$ foram geradas de acordo com o modelo descrito nesta seção. As taxas de rejeição para cada teste são apresentadas. Aparentemente a aproximação à distribuição nula assintótica não é boa para $n=20$, tanto para a estatística de distância quanto para a estatística de Wald. Pode-se observar que as taxas de rejeição sob $H_{1}$ estão longe dos níveis nominais ( $5 \%$ e $\left.10 \%\right)$. Para $n=50$ aparentemente não há problemas neste sentido. As taxas de rejeição na hipótese alternativa são sempre maiores para o teste de distância. Isto nos leva a crer que a utilização deste último teste neste caso é vantajosa, principalmente se levarmos em conta que os valores da estatística de distância e da probabilidade de significância podem ser obtidos através de formas explícitas bastante simples. 
Tabela 3.4: Taxas de Rejeição para os Testes de Distância e de Wald $\left(H_{1}: \beta_{1}=\beta_{2}=\beta_{3}=1, K_{1}:\left(\beta_{1}, \beta_{2}, \beta_{3}\right)^{\prime} \geq\right.$ $\left.(1,1,1)^{\prime}\right)$

\begin{tabular}{ccccccc}
\hline & \multicolumn{4}{c}{ Nível: $5 \%$} & \multicolumn{2}{c}{ Nível: $10 \%$} \\
\hline$n$ & $\beta_{2}$ & $\beta_{3}$ & \multicolumn{2}{c}{ Teste } & \multicolumn{2}{c}{ Teste } \\
& & & Distância & Wald & Distância & Wald \\
\hline 20 & 1.00 & 1.00 & 0.0870 & 0.0860 & 0.1550 & 0.1450 \\
& 1.00 & 1.02 & 0.0890 & 0.0740 & 0.1545 & 0.1290 \\
& 1.00 & 1.04 & 0.1125 & 0.0855 & 0.1745 & 0.1405 \\
1.00 & 1.06 & 0.1160 & 0.0895 & 0.1945 & 0.1545 \\
1.00 & 1.08 & 0.1250 & 0.1040 & 0.2125 & 0.1585 \\
1.00 & 1.10 & 0.1515 & 0.1160 & 0.2480 & 0.1830 \\
1.00 & 1.12 & 0.1665 & 0.1200 & 0.2665 & 0.1935 \\
1.00 & 1.14 & 0.2140 & 0.1590 & 0.3025 & 0.2405 \\
1.00 & 1.16 & 0.2250 & 0.1740 & 0.3345 & 0.2485 \\
1.00 & 1.18 & 0.2480 & 0.1890 & 0.3755 & 0.2750 \\
1.00 & 1.20 & 0.2905 & 0.2220 & 0.4205 & 0.3120 \\
\hline 1.02 & 1.02 & 0.1125 & 0.0975 & 0.1880 & 0.1495 \\
1.02 & 1.04 & 0.0985 & 0.0860 & 0.1745 & 0.1380 \\
1.02 & 1.06 & 0.1345 & 0.0975 & 0.2145 & 0.1595 \\
1.02 & 1.08 & 0.1375 & 0.1035 & 0.2270 & 0.1620 \\
1.02 & 1.10 & 0.1730 & 0.1235 & 0.2625 & 0.1815 \\
\hline 1.04 & 1.04 & 0.1250 & 0.0890 & 0.2075 & 0.1445 \\
1.04 & 1.06 & 0.1415 & 0.0915 & 0.2265 & 0.1520 \\
1.04 & 1.08 & 0.1295 & 0.0970 & 0.2205 & 0.1605 \\
1.04 & 1.10 & 0.1725 & 0.1195 & 0.2780 & 0.1875 \\
\hline 1.10 & 1.10 & 0.2305 & 0.1515 & 0.3255 & 0.2290 \\
1.10 & 1.12 & 0.2415 & 0.1605 & 0.3480 & 0.2415 \\
1.10 & 1.14 & 0.2810 & 0.1940 & 0.3945 & 0.2770 \\
1.10 & 1.16 & 0.3015 & 0.2135 & 0.4245 & 0.3025 \\
1.10 & 1.18 & 0.3400 & 0.2315 & 0.4735 & 0.3325 \\
\hline 50 & 1.00 & 1.00 & 0.0580 & 0.0525 & 0.1080 & 0.1010 \\
1.00 & 1.02 & 0.0850 & 0.0625 & 0.1550 & 0.1175 \\
\hline & & & continua & na próxima página \\
\hline & & & & & &
\end{tabular}




\begin{tabular}{cccccc}
\hline \multicolumn{7}{c}{ continuação da página anterior } \\
\hline \multicolumn{6}{c}{ Nível: $5 \%$} \\
\multicolumn{2}{c}{ Teste } & \multicolumn{2}{c}{ Testel: $10 \%$} \\
\hline$\beta_{2}$ & $\beta_{3}$ & Distância & Wald & Distância & Wald \\
\hline 1.00 & 1.04 & 0.0875 & 0.0720 & 0.1705 & 0.1225 \\
1.00 & 1.06 & 0.1170 & 0.0785 & 0.1950 & 0.1375 \\
1.00 & 1.08 & 0.1550 & 0.1035 & 0.2475 & 0.1755 \\
1.00 & 1.10 & 0.2055 & 0.1485 & 0.3160 & 0.2280 \\
1.00 & 1.12 & 0.2400 & 0.1645 & 0.3565 & 0.2660 \\
1.00 & 1.14 & 0.2940 & 0.1995 & 0.4070 & 0.3080 \\
1.00 & 1.16 & 0.3455 & 0.2515 & 0.4845 & 0.3695 \\
1.00 & 1.18 & 0.4575 & 0.3510 & 0.5850 & 0.4735 \\
1.00 & 1.20 & 0.5295 & 0.4245 & 0.6470 & 0.5345 \\
\hline 1.02 & 1.02 & 0.0940 & 0.0635 & 0.1645 & 0.1220 \\
1.02 & 1.04 & 0.1165 & 0.0765 & 0.2010 & 0.1295 \\
1.02 & 1.06 & 0.1370 & 0.0875 & 0.2295 & 0.1560 \\
1.02 & 1.08 & 0.1585 & 0.1040 & 0.2630 & 0.1775 \\
1.02 & 1.10 & 0.2015 & 0.1340 & 0.3230 & 0.2150 \\
\hline 1.04 & 1.04 & 0.1250 & 0.0695 & 0.2185 & 0.1305 \\
1.04 & 1.06 & 0.1610 & 0.1010 & 0.2535 & 0.1780 \\
1.04 & 1.08 & 0.1915 & 0.1190 & 0.3090 & 0.1985 \\
1.04 & 1.10 & 0.2285 & 0.1395 & 0.3535 & 0.2330 \\
\hline 1.10 & 1.10 & 0.3320 & 0.2045 & 0.4690 & 0.3165 \\
1.10 & 1.12 & 0.3755 & 0.2380 & 0.5315 & 0.3535 \\
1.10 & 1.14 & 0.4315 & 0.2815 & 0.5810 & 0.4050 \\
1.10 & 1.16 & 0.4845 & 0.3295 & 0.6355 & 0.4565 \\
1.10 & 1.18 & 0.5665 & 0.4075 & 0.6890 & 0.5360 \\
\hline
\end{tabular}

Para hipóteses mais complexas que $H_{1}$ e $K_{1}$, o esforço computacional para obtenção dos valores da estatística de distância e da probabilidade de significância pode ser maior. Suponha que desejamos testar

$$
H: h(\beta)=0 \text { contra } K: h(\beta) \geq 0 .
$$

A estatística de distância é dada por

$$
\mathbf{D}=\left\|\pi_{\Delta_{\beta}}\left(N_{j}^{1 / 2} h(\tilde{\beta}), \mathcal{O}_{l}\right)\right\|_{\Delta_{\beta}}^{2},
$$


onde $l$ é a dimensão do vetor $h(\beta)$,

$$
\Delta_{\beta}=J(\tilde{\beta}) \Omega_{\beta}(\tilde{\theta}) J(\tilde{\beta})^{\prime}
$$

e $J(\tilde{\beta})$ é a matriz jacobiana de $h$ avaliada em $\tilde{\beta}$.

Para obter $\mathbf{D}$, ou outras formas da estatística de distância que aparecerão nesta tese, somos obrigados a recorrer a algoritmos especializados. Observe também que, em geral, a distribuição nula assintótica de $\mathbf{D}$ depende do parâmetro associado ao modelo, de modo que o procedimento de teste que deve ser utilizado é o descrito após a demonstração do Teorema 2.2.4. Ou seja, o valor crítico do teste de nível $\xi$, $c_{\xi}$, deve ser estimado pela fórmula

$$
\sum_{i=1}^{l} P\left(\chi_{i}^{2} \geq c_{\xi}\right) \omega\left(l, i, \Delta_{\beta}\right)=\xi .
$$

Os apêndices A e B contêm uma descrição das técnicas computacionais utilizadas para uma correta implementação do teste, incluindo fórmulas para os pesos $\omega$.

Suponha, por exemplo, que temos um modelo com $k=4$ e estamos interessados em testar

$$
H_{2}: \beta_{1}=\beta_{2}=\beta_{3}=\beta_{4} \text { contra } K_{2}: \beta_{1} \leq \beta_{2} \leq \beta_{3} \leq \beta_{4}
$$

A tabela 3.5 apresenta um estudo de simulação nos mesmos moldes do anterior. Observe que $h(\beta)=\left(\beta_{2}-\beta_{1}, \beta_{3}-\beta_{2}, \beta_{4}-\beta_{3}\right)^{\prime}$. Tomamos $n_{1}=n_{2}=n_{3}=n_{4}=n$, $\sigma_{x 1}^{2}=\sigma_{x 2}^{2}=\sigma_{x 3}^{2}=\sigma_{x 4}^{2}=0.1, \sigma_{\delta 2}^{2}=\sigma_{\delta 3}^{3}=0.05, \sigma_{\delta 1}^{2}=0.01, \sigma_{\delta 4}^{2}=0.03, \sigma_{\epsilon 1}^{2}=\sigma_{\epsilon 2}^{2}=$ $\sigma_{\epsilon 3}^{2}=\sigma_{\epsilon 4}^{2} 0.50, \mu_{1}=\mu_{2}=\mu_{3}=\mu_{4}=1, \beta_{1}=\beta_{2}=1$.

Na tabela 3.6 apresentamos taxas de rejeição para diversos valores de $\beta=\beta_{1}=$ $\beta_{2}=\beta_{3}=\beta_{4}$. A aproximação da distribuição da estatística de distância à mistura de qui-quadrados parece ser muito boa, mesmo para $n=20$. 
Tabela 3.5: Taxas de Rejeição para os Testes de Distância e de Wald $\left(H_{2}: \beta_{1}=\beta_{2}=\beta_{3}=\beta_{4}, K_{2}: \beta_{1} \leq \beta_{2} \leq\right.$ $\left.\beta_{3} \leq \beta_{4}\right)$

\begin{tabular}{ccccccc}
\hline & \multicolumn{3}{c}{ Nível: $5 \%$} & \multicolumn{2}{c}{ Nível: $10 \%$} \\
\hline$n$ & $\beta_{3}$ & $\beta_{4}$ & \multicolumn{2}{c}{ Teste } & \multicolumn{2}{c}{ Teste } \\
& & & Distância & Wald & Distância & Wald \\
\hline 20 & 1.00 & 1.00 & 0.0580 & 0.0640 & 0.1080 & 0.1195 \\
& 1.00 & 1.02 & 0.0645 & 0.0725 & 0.1165 & 0.1225 \\
& 1.00 & 1.04 & 0.0770 & 0.0625 & 0.1365 & 0.1195 \\
1.00 & 1.06 & 0.0935 & 0.0690 & 0.1690 & 0.1140 \\
1.00 & 1.08 & 0.1015 & 0.0705 & 0.1850 & 0.1305 \\
1.00 & 1.10 & 0.1260 & 0.0870 & 0.2050 & 0.1535 \\
1.00 & 1.12 & 0.1310 & 0.0935 & 0.2230 & 0.1565 \\
1.00 & 1.14 & 0.1510 & 0.1025 & 0.2505 & 0.1705 \\
1.00 & 1.16 & 0.1790 & 0.1105 & 0.2855 & 0.1855 \\
1.00 & 1.18 & 0.2120 & 0.1340 & 0.3220 & 0.2215 \\
1.00 & 1.20 & 0.2410 & 0.1355 & 0.3515 & 0.2245 \\
\hline 1.02 & 1.02 & 0.0635 & 0.0595 & 0.1185 & 0.1185 \\
1.02 & 1.04 & 0.0785 & 0.0670 & 0.1390 & 0.1245 \\
1.02 & 1.06 & 0.0845 & 0.0685 & 0.1560 & 0.1260 \\
1.02 & 1.08 & 0.0965 & 0.0715 & 0.1655 & 0.1235 \\
1.02 & 1.10 & 0.1220 & 0.0730 & 0.2110 & 0.1330 \\
1.02 & 1.12 & 0.1270 & 0.0810 & 0.2200 & 0.1495 \\
1.02 & 1.14 & 0.1530 & 0.0905 & 0.2555 & 0.1540 \\
1.02 & 1.16 & 0.1790 & 0.1055 & 0.2910 & 0.1740 \\
1.02 & 1.18 & 0.1925 & 0.1060 & 0.3075 & 0.1755 \\
1.02 & 1.20 & 0.2265 & 0.1370 & 0.3245 & 0.2160 \\
\hline 1.04 & 1.04 & 0.0740 & 0.0600 & 0.1320 & 0.1200 \\
1.04 & 1.06 & 0.1085 & 0.0755 & 0.1815 & 0.1365 \\
1.04 & 1.08 & 0.1065 & 0.0695 & 0.1805 & 0.1230 \\
1.04 & 1.10 & 0.1200 & 0.0740 & 0.2005 & 0.1310 \\
1.04 & 1.12 & 0.1360 & 0.0720 & 0.2300 & 0.1390 \\
1.04 & 1.14 & 0.1595 & 0.0935 & 0.2585 & 0.1645 \\
\hline & & & & &
\end{tabular}




\begin{tabular}{cccccc}
\hline \multicolumn{7}{c}{ continuação da página anterior } \\
\hline \multicolumn{6}{c}{ Nível: $5 \%$} \\
\multicolumn{3}{c}{ Teste } & \multicolumn{2}{c}{ Tével: $10 \%$} \\
\hline$\beta_{2}$ & $\beta_{3}$ & Distância & Wald & Distância & Wald \\
\hline 1.04 & 1.16 & 0.1765 & 0.1160 & 0.2715 & 0.1720 \\
1.04 & 1.18 & 0.2075 & 0.1235 & 0.3235 & 0.1960 \\
1.04 & 1.20 & 0.2270 & 0.1270 & 0.3380 & 0.2070 \\
\hline 1.10 & 1.10 & 0.1425 & 0.0895 & 0.2300 & 0.1550 \\
1.10 & 1.12 & 0.1530 & 0.0895 & 0.2590 & 0.1695 \\
1.10 & 1.14 & 0.1720 & 0.0895 & 0.2790 & 0.1685 \\
1.10 & 1.16 & 0.2040 & 0.1120 & 0.3115 & 0.1930 \\
1.10 & 1.18 & 0.2150 & 0.1140 & 0.3330 & 0.1960 \\
1.10 & 1.20 & 0.2500 & 0.1420 & 0.3625 & 0.2255 \\
\hline 50 & 1.00 & 0.0495 & 0.0515 & 0.0985 & 0.0955 \\
1.00 & 1.02 & 0.0695 & 0.0575 & 0.1305 & 0.1045 \\
1.00 & 1.04 & 0.0780 & 0.0575 & 0.1525 & 0.1105 \\
1.00 & 1.06 & 0.0960 & 0.0630 & 0.1825 & 0.1165 \\
1.00 & 1.08 & 0.1275 & 0.0795 & 0.2145 & 0.1420 \\
1.00 & 1.10 & 0.1675 & 0.0940 & 0.2785 & 0.1705 \\
1.00 & 1.12 & 0.1980 & 0.1100 & 0.3190 & 0.1900 \\
1.00 & 1.14 & 0.2645 & 0.1620 & 0.3900 & 0.2520 \\
1.00 & 1.16 & 0.2895 & 0.1845 & 0.4340 & 0.2740 \\
1.00 & 1.18 & 0.3600 & 0.2175 & 0.4870 & 0.3265 \\
1.00 & 1.20 & 0.3910 & 0.2505 & 0.5330 & 0.3570 \\
\hline 1.02 & 1.02 & 0.0640 & 0.0515 & 0.1305 & 0.1005 \\
1.02 & 1.04 & 0.0855 & 0.0605 & 0.1525 & 0.1175 \\
1.02 & 1.06 & 0.1095 & 0.0710 & 0.1915 & 0.1230 \\
1.02 & 1.08 & 0.1305 & 0.0740 & 0.2155 & 0.1465 \\
1.02 & 1.10 & 0.1745 & 0.1005 & 0.2735 & 0.1635 \\
1.02 & 1.12 & 0.2040 & 0.1085 & 0.3085 & 0.1830 \\
1.02 & 1.14 & 0.2365 & 0.1420 & 0.3595 & 0.2170 \\
1.02 & 1.16 & 0.2810 & 0.1570 & 0.4205 & 0.2570 \\
1.02 & 1.18 & 0.3415 & 0.2030 & 0.4825 & 0.3090 \\
1.02 & 1.20 & 0.4080 & 0.2525 & 0.5525 & 0.3705 \\
\hline & & & continua na próxima página \\
\hline & & & & & \\
\hline & &
\end{tabular}




\begin{tabular}{cccccc}
\hline \multicolumn{1}{c}{ continuação da página anterior } \\
\hline \multicolumn{4}{c}{ Nível: $5 \%$} & \multicolumn{2}{c}{ Nível: $10 \%$} \\
\hline$\beta_{2}$ & $\beta_{3}$ & \multicolumn{2}{c}{ Teste } & \multicolumn{2}{c}{ Teste } \\
& & Distância & Wald & Distância & Wald \\
\hline 1.04 & 1.04 & 0.0815 & 0.0605 & 0.1625 & 0.1145 \\
1.04 & 1.06 & 0.1045 & 0.0645 & 0.1895 & 0.1115 \\
1.04 & 1.08 & 0.1385 & 0.0770 & 0.2240 & 0.1380 \\
1.04 & 1.10 & 0.1745 & 0.0850 & 0.2680 & 0.1530 \\
1.04 & 1.12 & 0.2135 & 0.1060 & 0.3320 & 0.1800 \\
1.04 & 1.14 & 0.2570 & 0.1445 & 0.3965 & 0.2260 \\
1.04 & 1.16 & 0.2960 & 0.1715 & 0.4170 & 0.2640 \\
1.04 & 1.18 & 0.3310 & 0.1875 & 0.4690 & 0.2765 \\
1.04 & 1.20 & 0.4135 & 0.2360 & 0.5530 & 0.3485 \\
\hline 1.10 & 1.10 & 0.1970 & 0.1025 & 0.3105 & 0.1835 \\
1.10 & 1.12 & 0.2425 & 0.1275 & 0.3555 & 0.1935 \\
1.10 & 1.14 & 0.2770 & 0.1435 & 0.4120 & 0.2315 \\
1.10 & 1.16 & 0.3110 & 0.1610 & 0.4675 & 0.2605 \\
1.10 & 1.18 & 0.3755 & 0.1895 & 0.5275 & 0.3115 \\
1.10 & 1.20 & 0.4185 & 0.2360 & 0.5620 & 0.3485 \\
\hline
\end{tabular}


Tabela 3.6: Taxas de Rejeição sob a Hipótese Nula para os Testes de Distância e de Wald, $H_{2}$ contra $K_{2}$.

\begin{tabular}{cccccc}
\hline & \multicolumn{2}{c}{ Nível: $5 \%$} & \multicolumn{2}{c}{ Nível: $10 \%$} \\
\hline$n$ & $\beta$ & \multicolumn{2}{c}{ Teste } & \multicolumn{2}{c}{ Teste } \\
& & Distância & Wald & Distância & Wald \\
\hline 20 & 1.4 & 0.0575 & 0.0710 & 0.1115 & 0.1335 \\
& 2.0 & 0.0505 & 0.0620 & 0.1020 & 0.1175 \\
& 2.4 & 0.0495 & 0.0650 & 0.0975 & 0.1160 \\
& 3.0 & 0.0540 & 0.0675 & 0.1020 & 0.1170 \\
& 4.0 & 0.0475 & 0.0595 & 0.0965 & 0.1045 \\
\hline 50 & 1.4 & 0.0430 & 0.0520 & 0.0850 & 0.1040 \\
& 2.0 & 0.0505 & 0.0540 & 0.1015 & 0.1035 \\
& 2.4 & 0.0450 & 0.0495 & 0.0875 & 0.0985 \\
& 3.0 & 0.0515 & 0.0660 & 0.0965 & 0.1170 \\
& 4.0 & 0.0470 & 0.0665 & 0.1030 & 0.1175 \\
\hline
\end{tabular}




\section{Capítulo 4}

\section{O Modelo de Regressão Linear Múltiplo Multivariado}

Neste capítulo tratamos do modelo de regressão linear múltiplo multivariado com erros nos regressores e matriz de covariância dos erros estimada independentemente. Apresentamos uma solução para a estimação de máxima verossimilhança e mostramos que esta é consistente e assintoticamente normal. Com isto, podemos desenvolver o teste de distância para hipóteses relevantes. Estudos de poder, com dados simulados, são feitos para fins de comparação com testes tradicionais, mas inadequados para a situação.

\subsection{Definição do Modelo e Estimação de Máxima Verossimilhança}

Consideremos a seguinte extensão multivariada do modelo definido na Seção 3.1.1: $\left\{z_{i}, i \in \mathbb{N}\right\}$ é um conjunto de vetores aleatórios não observáveis de dimensão $p$, $z_{i}=\left(x^{\prime}{ }_{i}, y_{i}^{\prime}\right)^{\prime}$, onde $x_{i}: k \times 1$ e $y_{i}: r \times 1 . x_{i}$ e $y_{i}$ satisfazem

$$
y_{i}=\alpha+\beta x_{i}, \quad i \in \mathbb{N}
$$

onde $\alpha: r \times 1$ e $\beta: r \times k$ são matrizes de parâmetros a serem estimados. As observações constituem o conjunto $\left\{Z_{i}, i \in \mathbb{N}\right\}$, onde $Z_{i}=\left(X^{\prime}{ }_{i}, Y^{\prime}{ }_{i}\right)^{\prime}$ é definido por

$$
Z_{i}=z_{i}+\epsilon_{i}, \quad i \in \mathbb{N},
$$


onde $\epsilon_{i}=\left(\delta^{\prime}{ }_{i}, e^{\prime}{ }_{i}\right)^{\prime}, \delta_{i}: k \times 1$, é um vetor de erros de observação. Vamos supor que

$$
\left\{\left(x^{\prime}{ }_{i}, \epsilon_{i}^{\prime}\right)^{\prime}, i \in \mathbb{N}\right\} \sim N I I D\left[\left(\mu_{x}^{\prime}, 0\right)^{\prime} \text {, block } \operatorname{diag}\left\{\Sigma_{x}, \Sigma_{\epsilon}\right\}\right],
$$

e que $\Sigma_{x}>0, \Sigma_{\epsilon}>0$.

Definimos o modelo de regressão linear múltipla multivariado com erros nas variáveis por (4.1), (4.2) e (4.3). Por (4.1) e (4.2), temos que

$$
Z_{i}=\left(\begin{array}{ccc}
I_{k} & I_{k} & 0 \\
\beta & 0 & I_{r}
\end{array}\right)\left(\begin{array}{c}
x_{i} \\
\delta_{i} \\
e_{i}
\end{array}\right)+\left(\begin{array}{c}
0 \\
\alpha
\end{array}\right), i \in \mathbb{N}
$$

de modo que

$$
\left\{Z_{i}, i \in \mathbb{N}\right\} \sim N I I D(\mu(\theta), \Sigma(\theta))
$$

onde $\theta=\left(\mu_{x}, \alpha, \beta, \Sigma_{x}, \Sigma_{\epsilon}\right)$,

$$
\begin{aligned}
\mu(\theta) & =\left(\begin{array}{c}
\mu_{x} \\
\alpha+\beta \mu_{x}
\end{array}\right), \\
\Sigma(\theta) & =\Sigma_{z}(\theta)+\Sigma_{\epsilon}, \\
\Sigma_{z}(\theta) & =\left(\begin{array}{cc}
\Sigma_{x} & \Sigma_{x} \beta^{\prime} \\
\beta \Sigma_{x} & \beta \Sigma_{x} \beta^{\prime}
\end{array}\right) .
\end{aligned}
$$

Observe que $\Sigma(\theta)$ é positiva definida e $\Sigma_{z}(\theta)$ é de posto $k$, pois

$$
\Sigma_{z}(\theta)=\left(I, \beta^{\prime}\right)^{\prime} \Sigma_{x}\left(I, \beta^{\prime}\right)
$$

No capítulo 3 observamos que, para $r=k=1$, o modelo definido por (4.5)(4.8) não é identificável. Isto também é verdadeiro em dimensões maiores, de modo que precisamos de hipóteses adicionais que garantam identificabilidade. No capítulo 5 faremos a suposição de que $\Sigma_{\epsilon}$ é igual ao produto de uma constante positiva e desconhecida por uma matriz positiva definida conhecida. Isto é uma extensão da suposição de razão de variâncias dos erros conhecida, veja a seção 3.1.3. Neste capítulo, vamos supor a existência de uma sequência de estimadores de $\Sigma_{\epsilon}$ consistente, $\left\{S_{d}, d \in \mathbb{N}, d>p\right\}$, independente de $\left\{Z_{i}, i \in \mathbb{N}\right\}$, tal que

$$
d S_{d} \sim W\left(d, \Sigma_{\epsilon}\right)
$$


onde $W\left(d, \Sigma_{\epsilon}\right)$ denota a distribuição de Wishart com $d$ graus de liberdade e parâmetro $\Sigma_{\epsilon}$. (4.10) mais (4.5)-(4.8) definem o modelo com o qual trabalharemos. Como a matriz de covariâncias dos erros de observação é estimada, o modelo será referenciado como MRME. O espaço paramétrico associado é dado por

$$
\Theta^{*}=\left\{\left(\mu_{x}, \alpha, \beta, \Sigma_{x}, \Sigma_{\epsilon}\right) ; \mu_{x} \in \mathbb{R}^{p}, \alpha \in \mathbb{R}^{p}, \beta: r \times k, \Sigma_{x} \in \mathcal{M}_{k}, \Sigma_{\epsilon} \in \mathcal{M}_{p}\right\} .
$$

Enunciaremos dois lemas que serão úteis na estimação de máxima verossimilhança. Seja $A: s \times s$ uma matriz simétrica. Quando escrevermos " $B$ é uma matriz de autovetores de $A$ associados à matriz de autovalores $\Lambda$ ". queremos dizer que $\Lambda=\operatorname{diag}\left\{\lambda_{1}, \ldots, \lambda_{s}\right\}$, onde $\lambda_{i}$ é autovalor de $A, \lambda_{1} \geq \ldots \geq \lambda_{s}$, e $B$ é uma matriz cuja $i$-ésima coluna é um autovetor associado a $\lambda_{i}$.

Lema 4.1.1 Sejam $A: s \times s$ simétrica e $B \in \mathcal{M}_{s}$. Então existem matrizes $T: s \times s$, invertivel, e $\Lambda$, diagonal, tais que

$$
T^{\prime} A T=\Lambda, T^{\prime} B T=I .
$$

As matrizes $\Lambda$ e $T$ citadas no enunciado são obtidas da seguinte maneira:

$$
\Lambda=\operatorname{diag}\left\{\lambda_{1}, \ldots, \lambda_{s}\right\}
$$

onde $\lambda_{1} \geq \ldots \geq \lambda_{s}$ são as raízes do polinômio em $\lambda$

$$
|A-\lambda B|=0
$$

que são reais, pois são autovalores da matriz $B^{-1 / 2} A B^{-1 / 2}$, que é simétrica. Seja $Q$ uma matriz ortogonal de autovetores de $B^{-1 / 2} A B^{-1 / 2}$ associada à $\Lambda$. Então

$$
B^{-1 / 2} A B^{-1 / 2} Q=Q \Lambda \text {. }
$$

Tomamos então

$$
T=B^{-1 / 2} Q
$$

$\lambda_{i}, i=1, \ldots, s$ são denominados autovalores de $A$ na métrica $B$. O $i$-ésimo vetor coluna de $T$ é denominado autovetor de $A$ na métrica $B$ associado ao autovalor $\lambda_{i}$.

A demonstração do lema a seguir pode ser encontrada em Fuller (1987, pg. 395). 
Lema 4.1.2 Sejam $A: s \times s$ e $B: s \times s$ simétricas, com $|B| \neq 0$. Sejam $\lambda_{1} \geq \ldots \geq$ $\lambda_{s}$ os autovalores de $A, \nu_{1} \geq \ldots \geq \nu_{s}$ os autovalores de $B$,

$$
\Lambda=\operatorname{diag}\left\{\lambda_{1}, \ldots, \lambda_{s}\right\}, V=\operatorname{diag}\left\{\nu_{1}, \ldots, \nu_{s}\right\}
$$

Então,

$$
\operatorname{tr}\left(A B^{-1}\right) \geq \operatorname{tr}\left(\Lambda V^{-1}\right) .
$$

A igualdade vale se e somente se existe uma matriz ortogonal $U$ tal que

$$
U^{\prime} A U=\Lambda, U^{\prime} B U=V
$$

Consideremos a estimação de máxima verossimilhança dos parâmetros do MRME. Defina

$$
\Phi=\left\{(\Sigma, \Omega) \in \mathcal{M}_{p} \times \mathcal{M}_{p} ; \Sigma-\Omega \geq 0, \operatorname{posto}(\Sigma-\Omega)=k\right\}
$$

Considere a verossimilhança irrestrita, ou seja, a verossimilhança oriunda do modelo definido por $\left\{Z_{1}, \ldots, Z_{n}\right\} \sim N I I D(\mu, \Sigma)$ e $d S_{d} \sim W(d, \Omega)$ independentes, $(\mu, \Sigma, \Omega) \in \mathbb{R}^{p} \times \Phi, d>p$ (para $\left.\theta \in \Theta^{*},\left(\mu(\theta), \Sigma(\theta), \Sigma_{\epsilon}\right) \in \mathbb{R}^{p} \times \Phi\right)$. A idéia é maximizar a verossimilhança irrestrita em $\mathbb{R}^{p} \times \Phi$, encontrando um ponto de máximo global $(\hat{\mu}, \hat{\Sigma}, \hat{\Omega})$, e então resolver o sistema em $\theta$

$$
\left(\mu(\theta), \Sigma(\theta), \Sigma_{\epsilon}\right)=(\hat{\mu}, \hat{\Sigma}, \hat{\Omega}), \theta \in \Theta^{*}
$$

A solução será o estimador de máxima verossimilhança.

No que segue, omitimos subscritos em sequências de estimadores e escrevemos $S$ em vez de $S_{d}$, por exemplo. A log-verossimilhança irrestrita é dada por

$$
\begin{aligned}
(\mu, \Sigma, \Omega) \mapsto & -\frac{n}{2}\left[\log |\Sigma|+\operatorname{tr}\left(S_{Z} \Sigma^{-1}\right)+(\bar{Z}-\mu)^{\prime} \Sigma^{-1}(\bar{Z}-\mu)\right] \\
& -\frac{d}{2}\left[\operatorname{tr}\left(\Omega^{-1} S\right)+\log |\Omega|\right], \quad(\mu, \Sigma, \Omega) \in \mathbb{R}^{p} \times \Phi .
\end{aligned}
$$

Pelo corolário 3.4.8.1 de Mardia et al. (1979), $S>0$ com probabilidade 1. Pelo Lema 4.1.1, existe (com probabilidade 1) $T: p \times p$ invertível tal que

$$
T^{\prime} S_{Z} T=\hat{\Lambda}, T^{\prime} S T=I_{p}
$$


onde $\hat{\Lambda}=\operatorname{diag}\left\{\hat{\lambda}_{1}, \ldots, \hat{\lambda}_{p}\right\}, \hat{\lambda}_{1} \geq \ldots \geq \hat{\lambda}_{p}$. Fazendo $\Sigma_{T}=T^{\prime} \Sigma T, \Omega_{T}=T^{\prime} \Omega T$, e observando que

$$
\begin{aligned}
|\Sigma| & =\left|\Sigma_{T}\right|\left|\left(T^{\prime}\right)^{-1} T^{-1}\right|=\left|\Sigma_{T}\right||S| \\
|\Omega| & =\left|\Omega_{T}\right||S| \\
\operatorname{tr}\left(S_{Z} \Sigma^{-1}\right) & =\operatorname{tr}\left[\left(T^{\prime}\right)^{-1} \hat{\Lambda} T^{-1} T \Sigma_{T}^{-1} T^{\prime}\right] \\
& =\operatorname{tr}\left(\hat{\Lambda} \Sigma_{T}^{-1}\right), \\
\operatorname{tr}\left(S \Omega^{-1}\right) & =\operatorname{tr}\left[\left(T^{\prime}\right)^{-1} T^{-1} T \Omega_{T}^{-1} T^{\prime}\right] \\
& =\operatorname{tr}\left(\Omega_{T}^{-1}\right),
\end{aligned}
$$

temos que o problema de maximização da log-verossimilhança irrestrita é equivalente ao problema de maximização da log-verossimilhança reparametrizada $G: \mathbb{R}^{p} \times \Phi \rightarrow \mathbb{R}$ dada por

$$
\begin{aligned}
G\left(\mu, \Sigma_{T}, \Omega_{T}\right)= & -\frac{n}{2}\left[\log \left|\Sigma_{T}\right|+\log |S|+\operatorname{tr}\left(\hat{\Lambda} \Sigma_{T}^{-1}\right)+(\bar{Z}-\mu)^{\prime} T \Sigma_{T}^{-1} T^{\prime}(\bar{Z}-\mu)\right] \\
& -\frac{d}{2}\left[\log \left|\Omega_{T}\right|+\log |S|+\operatorname{tr}\left(\Omega_{T}^{-1}\right)\right] .
\end{aligned}
$$

$\mu$ e $\left(\Sigma_{T}, \Omega_{T}\right)$ são variacionalmente independentes. Podemos então aplicar o princípio do supremo iterado. Fixando $\Sigma_{T}$ e $\Omega_{T}$ temos que a função parcial $G_{\Sigma_{T}, \Omega_{T}}$ é claramente maximizada em $\hat{\mu}=\bar{Z}$. A função parcial $G_{\bar{Z}}$ é dada por

$$
G_{\bar{Z}}\left(\Sigma_{T}, \Omega_{T}\right)=-\frac{n}{2}\left[\log \left|\Sigma_{T}\right|+\operatorname{tr}\left(\hat{\Lambda} \Sigma_{T}^{-1}\right)\right]-\frac{d}{2}\left[\log \left|\Omega_{T}\right|+\operatorname{tr}\left(\Omega_{T}^{-1}\right)\right],
$$

onde, sem perda de generalidade, eliminamos constantes aditivas.

O teorema a seguir mostra que maximimizar $G_{\bar{Z}}$ é equivalente a maximizar $\left.G_{\bar{Z}}\right|_{\Phi^{*}}$, onde

$$
\begin{aligned}
\Phi^{*}= & \left\{\left(P, P^{1 / 2} U^{-1} P^{1 / 2}\right) ; P=\operatorname{diag}\left\{\gamma_{1}, \ldots, \gamma_{p}\right\}, U=\operatorname{diag}\left\{u_{1}, \ldots, u_{k}, 1, \ldots, 1\right\},\right. \\
& \left.\gamma_{1} \geq \ldots \geq \gamma_{p}>0, u_{1} \geq \ldots \geq u_{k}>1\right\} .
\end{aligned}
$$

Teorema 4.1.1 Para todo $\left(\Sigma_{T}, \Omega_{T}\right) \in \Phi$, existe $\left(\Sigma_{T}^{*}, \Omega_{T}^{*}\right) \in \Phi^{*}$ tal que

$$
G_{\bar{Z}}\left(\Sigma_{T}^{*}, \Omega_{T}^{*}\right) \geq G_{\bar{Z}}\left(\Sigma_{T}, \Omega_{T}\right)
$$

Demonstração: Os autovalores de $\Sigma_{T}$ na métrica $\Omega_{T}, \nu_{1} \geq \ldots \geq \nu_{p}$, são soluções do polinômio em $\nu$

$$
\left|\Sigma_{T}-\Omega_{T}-(\nu-1) \Omega_{T}\right|=0 \text {. }
$$


Assim, se $\lambda_{1} \geq \ldots \geq \lambda_{p}$ são os autovalores de $\Sigma_{T}-\Omega_{T}$ na métrica $\Omega_{T}$, então $\nu_{i}=1+\lambda_{i}, i=1, \ldots, p$. Como o posto de $\Sigma_{T}-\Omega_{T}$ é $k, \lambda_{i}, i=1, \ldots, k$ são todos positivos e $\lambda_{k+1}=\ldots=\lambda_{p}=0$. Ou seja, $\nu_{1} \geq \ldots \geq \nu_{k}>1$ e $\nu_{k+1}=\ldots=\nu_{p}=1$. Sejam $\gamma_{1} \geq \ldots \geq \gamma_{p}>0$ os autovalores usuais de $\Sigma_{T}$ e defina

$$
P=\operatorname{diag}\left\{\gamma_{1}, \ldots, \gamma_{p}\right\}, V=\operatorname{diag}\left\{\nu_{1}, \ldots, \nu_{k}, 1, \ldots, 1\right\}
$$

Façamos $\Sigma_{T}^{*}=P, \Omega_{T}^{*}=P^{1 / 2} V^{-1} P^{1 / 2}$.

$\nu_{i}^{-1}, i=1, \ldots, k$ e 1 , com multiplicidade algébrica $p-k$, são os autovalores de $\Omega_{T}$ na métrica $\Sigma_{T}$. Pelo Lema 4.1.1 existe uma matriz $W$ invertível tal que

$$
\Omega_{T}=W V^{-1} W^{\prime}, \quad \Sigma_{T}=W W^{\prime} .
$$

Assim,

$$
\begin{aligned}
\log \left|\Omega_{T}\right| & =\log \left|\Sigma_{T}\right|+\log \left|V^{-1}\right| \\
\operatorname{tr}\left(\Omega_{T}^{-1}\right) & =\operatorname{tr}\left[W^{-1}\left(W^{-1}\right)^{\prime} V\right] .
\end{aligned}
$$

Substituindo em (4.16),

$$
\begin{aligned}
G_{\bar{Z}}\left(\Sigma_{T}, \Omega_{T}\right)= & -\frac{n}{2}\left[\log \left|\Sigma_{T}\right|+\operatorname{tr}\left(\hat{\Lambda} \Sigma_{T}^{-1}\right)\right] \\
& -\frac{d}{2}\left\{\log \left|\Sigma_{T}\right|+\log \left|V^{-1}\right|+\operatorname{tr}\left[W^{-1}\left(W^{-1}\right)^{\prime} V\right]\right\} .
\end{aligned}
$$

Pelo Teorema Espectral, $\left|\Sigma_{T}\right|=|P|$. Pelo Lema 4.1.2,

$$
\operatorname{tr}\left(\hat{\Lambda} \Sigma_{T}^{-1}\right) \geq \operatorname{tr}\left(\hat{\Lambda} P^{-1}\right), \operatorname{tr}\left[W^{-1}\left(W^{-1}\right)^{\prime} V\right] \geq \operatorname{tr}\left(V P^{-1}\right),
$$

já que $\left(W^{-1}\right)^{\prime} W^{-1}=\Sigma_{T}^{-1}$ e os autovalores de $\left(W^{-1}\right)^{\prime} W^{-1}$ são os mesmos de $W^{-1}\left(W^{-1}\right)^{\prime}$. Assim,

$$
\begin{aligned}
G_{\bar{Z}}\left(\Sigma_{T}, \Omega_{T}\right) & \leq-\frac{n+d}{2} \log |P|-\frac{d}{2} \log \left|V^{-1}\right|-\frac{n}{2} \operatorname{tr}\left(\hat{\Lambda} P^{-1}\right)-\frac{d}{2} \operatorname{tr}\left(V P^{-1}\right) \\
& =G_{\bar{Z}}\left(\Sigma_{T}^{*}, \Omega_{T}^{*}\right) .
\end{aligned}
$$

Seja então $\left(\Sigma_{T}, \Omega_{T}\right) \in \Phi^{*}$,

$$
\begin{aligned}
\Sigma_{T} & =\operatorname{diag}\left\{\gamma_{1}, \ldots, \gamma_{p}\right\}, \Omega_{T}=\Sigma_{T}^{1 / 2} V^{-1} \Sigma_{T}^{1 / 2} \\
V & =\operatorname{diag}\left\{\nu_{1}, \ldots, \nu_{k}, 1, \ldots, 1\right\}
\end{aligned}
$$


$\gamma_{1} \geq \ldots \geq \gamma_{p}>0$ e $\nu_{1} \geq \ldots \geq \nu_{k}>1$. Temos que

$$
\begin{aligned}
G_{\bar{Z}}\left(\Sigma_{T}, \Omega_{T}\right)= & -\frac{n}{2}\left(\sum_{i=1}^{p} \log \gamma_{i}+\sum_{i=1}^{p} \hat{\lambda}_{i} \gamma_{i}^{-1}\right) \\
& -\frac{d}{2}\left(\sum_{i=1}^{p} \log \gamma_{i}+\sum_{i=1}^{k} \log \nu_{i}^{-1}+\sum_{i=1}^{k} \nu_{i} \gamma_{i}^{-1}+\sum_{i=k+1}^{p} \gamma_{i}^{-1}\right) \\
= & -\frac{n+d}{2} \sum_{i=1}^{p} \log \gamma_{i}-\frac{n}{2} \sum_{i=1}^{p} \hat{\lambda}_{i} \gamma_{i}^{-1} \\
& -\frac{d}{2}\left(\sum_{i=1}^{k} \log \nu_{i}^{-1}+\sum_{i=1}^{k} \nu_{i} \gamma_{i}^{-1}+\sum_{i=k+1}^{p} \gamma_{i}^{-1}\right) \\
= & -\frac{n+d}{2} \sum_{i=1}^{p} \log \gamma_{i}-\frac{n}{2} \sum_{i=1}^{k} \hat{\lambda}_{i} \gamma_{i}^{-1}-\frac{n}{2} \sum_{i=k+1}^{p} \hat{\lambda}_{i} \gamma_{i}^{-1} \\
& -\frac{d}{2} \sum_{i=1}^{k} \log \nu_{i}^{-1}-\frac{d}{2} \sum_{i=1}^{k} \nu_{i} \gamma_{i}^{-1}-\frac{d}{2} \sum_{i=k+1}^{p} \gamma_{i}^{-1} \\
= & -\frac{n+d}{2} \sum_{i=1}^{p} \log \gamma_{i}-\frac{1}{2} \sum_{i=1}^{k} \gamma_{i}^{-1}\left(n \hat{\lambda}_{i}+d \nu_{i}\right) \\
& -\frac{1}{2} \sum_{i=k+1}^{p} \gamma_{i}^{-1}\left(n \hat{\lambda}_{i}+d\right)-\frac{d}{2} \sum_{i=1}^{k} \log \nu_{i}^{-1} .
\end{aligned}
$$

Façamos $\gamma=\left(\gamma_{1}, \ldots, \gamma_{p}\right), \nu=\left(\nu_{1}, \ldots, \nu_{k}\right), h(\gamma, \nu)=G_{\bar{Z}}\left(\Sigma_{T}, \Omega_{T}\right)$. O problema agora reduz-se a maximização da função $h$, com domínio

$$
\operatorname{dom}(h)=\left\{(\gamma, \nu) \in \mathbb{R}^{p} \times \mathbb{R}^{k} ; \gamma_{1} \geq \ldots \geq \gamma_{p}>0, \nu_{1} \geq \ldots \geq \nu_{k}>1\right\} .
$$

Fixemos $\nu_{1} \geq \ldots \geq \nu_{k}>1$. Fazendo

$$
\partial_{1} h\left(\gamma_{1}, \ldots, \gamma_{p}, \nu_{1}, \ldots, \nu_{k}\right)=0
$$

temos que o único ponto crítico da função parcial $h_{\nu}$ é $\hat{\gamma}=\left(\hat{\gamma}_{1}, \ldots, \hat{\gamma}_{p}\right)$ dado por

$$
\hat{\gamma}_{i}=\left\{\begin{array}{lll}
\frac{n \hat{\lambda}_{i}+d \nu_{i}}{n+d}, & \text { se } & i=1, \ldots, k \\
\frac{n \hat{\lambda}_{i}+d}{n+d}, & \text { se } & i=k+1, \ldots, p
\end{array} .\right.
$$

Temos que

$$
\frac{\partial^{2} h_{\nu}}{\partial \gamma_{i}^{2}}\left(\gamma_{1}, \ldots, \gamma_{p}\right)=\frac{n+d}{2 \gamma_{i}^{2}}-\frac{n \hat{\lambda}_{i}+d \nu_{i}}{\gamma_{i}^{3}}=\frac{1}{\gamma_{i}^{2}}\left(\frac{n+d}{2}-\frac{n \hat{\lambda}_{i}+d \nu_{i}}{\gamma_{i}}\right),
$$


se $i=1, \ldots, k, \mathrm{e}$

$$
\frac{\partial^{2} h_{\nu}}{\partial \gamma_{i}^{2}}\left(\gamma_{1}, \ldots, \gamma_{p}\right)=\frac{n+d}{2 \gamma_{i}^{2}}-\frac{n \hat{\lambda}_{i}+d}{\gamma_{i}^{3}}=\frac{1}{\gamma_{i}^{2}}\left(\frac{n+d}{2}-\frac{n \hat{\lambda}_{i}+d}{\gamma_{i}}\right),
$$

se $i=k+1, \ldots, p$. Assim,

$$
\frac{\partial^{2} h_{\nu}}{\partial \gamma_{i}^{2}}(\hat{\gamma})=-\frac{n+d}{2 \hat{\gamma}_{i}^{2}}
$$

se $i=1, \ldots, p$ e

$$
\frac{\partial^{2} h_{\nu}}{\partial \gamma_{i} \partial \gamma_{j}}(\hat{\gamma})=0
$$

para $i \neq j$. Desta forma, a matriz Jacobiana de $h_{\nu}$ avaliada no ponto crítico $\hat{\gamma}$ é

$$
-\frac{n+d}{2} \operatorname{diag}\left\{\hat{\gamma}_{1}^{-2}, \ldots, \hat{\gamma}_{p}^{-2}\right\}
$$

que, com probabilidade 1 , é uma matriz negativa definida. Assim, $\hat{\gamma}$ é ponto de máximo global de $h_{\nu}$, já que $\hat{\lambda}_{1} \geq \ldots \geq \hat{\lambda}_{k}$ e $\nu_{1} \geq \ldots \geq \nu_{k}>1$ implicam em $\hat{\gamma}_{1} \geq \ldots \geq \hat{\gamma}_{p}>0$. Devemos agora maximizar a função parcial $h_{\hat{\gamma}}$. Temos que

$$
\begin{aligned}
h_{\hat{\gamma}}(\nu)= & -\frac{n+d}{2}\left(\sum_{i=1}^{k} \log \frac{n \hat{\lambda}_{i}+d \nu_{i}}{n+d}+\sum_{i=k+1}^{p} \log \hat{\gamma}_{i}\right) \\
& -\frac{1}{2} p(n+d)+\frac{d}{2} \sum_{i=1}^{k} \log \nu_{i} \\
= & -\frac{n+d}{2}\left(p+\sum_{i=k+1}^{p} \log \hat{\gamma}_{i}\right)-\frac{n+d}{2} \sum_{i=1}^{k} \log \frac{n \hat{\lambda}_{i}+d \nu_{i}}{n+d} \\
& +\frac{d}{2} \sum_{i=1}^{k} \log \nu_{i} \\
= & -\frac{n+d}{2}\left(p+\sum_{i=k+1}^{p} \log \hat{\gamma}_{i}\right)-\frac{1}{2} \sum_{i=1}^{k} \eta\left(\nu_{i}, \hat{\lambda}_{i}\right)
\end{aligned}
$$

onde

$$
\eta(\omega, \lambda)=(n+d) \log \frac{n \lambda+d \omega}{n+d}-d \log \omega .
$$

O problema reduz-se então em encontrar

$$
\max \left\{-\frac{1}{2} \sum_{i=1}^{k} \eta\left(\nu_{i}, \hat{\lambda}_{i}\right), \nu_{1}, \ldots, \nu_{k}>1\right\} .
$$


Dois casos devem ser considerados: quando $\hat{\lambda}_{k}>1$ e quando $\hat{\lambda}_{k} \leq 1$. Suponha que ocorra o primeiro caso. Para $\lambda>1$ fixo obtemos, por diferenciação,

$$
\inf \{\eta(\omega, \lambda) ; \omega>1\}=\eta(\lambda, \lambda)
$$

de modo que $\left(\hat{\nu}_{1}, \ldots, \hat{\nu}_{k}\right)=\left(\hat{\lambda}_{1}, \ldots, \hat{\lambda}_{k}\right)$ é ponto de máximo global de $h_{\hat{\gamma}}$, pois $\hat{\lambda}_{1} \geq \ldots \geq \hat{\lambda}_{k}>1$. O ponto de máximo global de $h$ é dado por

$$
\left(\hat{\gamma}_{1}, \ldots, \hat{\gamma}_{p}, \hat{\nu}_{1}, \ldots, \hat{\nu}_{k}\right)=\left(\hat{\lambda}_{1}, \ldots, \hat{\lambda}_{k}, \frac{n \hat{\lambda}_{k+1}+d}{n+d}, \ldots, \frac{n \hat{\lambda}_{p}+d}{n+d}, \hat{\lambda}_{1}, \ldots, \hat{\lambda}_{k}\right) .
$$

O ponto de máximo global de $G$ é $\left(\bar{Z}, \hat{\Sigma}_{T}, \hat{\Omega}_{T}\right)$, onde

$$
\begin{aligned}
\hat{\Sigma}_{T} & =\operatorname{diag}\left\{\hat{\lambda}_{1}, \ldots, \hat{\lambda}_{k}, \frac{n \hat{\lambda}_{k+1}+d}{n+d}, \ldots, \frac{n \hat{\lambda}_{p}+d}{n+d}\right\}, \\
\hat{\Omega}_{T} & =\hat{\Sigma}_{T}^{1 / 2} \hat{V}^{-1} \hat{\Sigma}_{T}^{1 / 2} \\
\hat{V}^{-1} & =\operatorname{diag}\left\{\hat{\lambda}_{1}, \ldots, \hat{\lambda}_{k}, 1, \ldots, 1\right\} .
\end{aligned}
$$

Assim,

$$
\hat{\Omega}_{T}=\operatorname{diag}\left\{1, \ldots, 1, \frac{n \hat{\lambda}_{k+1}+d}{n+d}, \ldots, \frac{n \hat{\lambda}_{p}+d}{n+d}\right\} .
$$

O máximo da verossimilhança irrestrita é então atingido no ponto $(\bar{Z}, \hat{\Sigma}, \hat{\Omega})$, onde

$$
\begin{aligned}
& \hat{\Sigma}=\left(T^{\prime}\right)^{-1} \hat{\Sigma}_{T} T^{-1}=S T \hat{\Sigma}_{T} T^{\prime} S \\
& \hat{\Omega}=S T \hat{\Omega}_{T} T^{\prime} S,
\end{aligned}
$$

igualdades que são obtidas a partir de (4.15). Finalmente, para encontrarmos a solução de máxima verossimilhança para o parâmetro $\theta$ no MRME, devemos encontrar $\hat{\theta}=\left(\hat{\mu}_{x}, \hat{\alpha}, \hat{\beta}, \hat{\Sigma}_{x}, \hat{\Sigma}_{\epsilon}\right) \in \Theta^{*}$ solução do sistema dado em (4.13). Assim,

$$
\hat{\mu}_{x}=\bar{X}, \hat{\alpha}+\hat{\beta} \bar{X}=\bar{Y}, \quad \hat{\Sigma}_{\epsilon}=\hat{\Omega},\left(\begin{array}{cc}
\hat{\Sigma}_{x} & \hat{\Sigma}_{x} \hat{\beta}^{\prime} \\
\hat{\beta} \hat{\Sigma}_{x} & \hat{\beta} \hat{\Sigma}_{x} \hat{\beta}^{\prime}
\end{array}\right)+\hat{\Sigma}_{\epsilon}=\hat{\Sigma} .
$$

Fazendo as partições

$$
\hat{\Sigma}=\left(\hat{\Sigma}_{i j}\right), \hat{\Omega}=\left(\hat{\Omega}_{i j}\right), i, j=1,2, \hat{\Sigma}_{11}, \hat{\Omega}_{11}: k \times k,
$$

obtemos

$$
\hat{\Sigma}_{x}=\hat{\Sigma}_{11}-\hat{\Omega}_{11}
$$


Note que

$$
T^{\prime}\left(\hat{\Sigma}-\hat{\Sigma}_{\epsilon}\right) T=\hat{\Sigma}_{T}-\hat{\Omega}_{T}=\operatorname{diag}\left\{\hat{\lambda}_{1}-1, \ldots, \hat{\lambda}_{k}-1,0, \ldots, 0\right\} .
$$

Assim,

$$
\begin{aligned}
\hat{\Sigma}-\hat{\Sigma}_{\epsilon} & =\left(T^{-1}\right)^{\prime} \operatorname{diag}\left\{\hat{\lambda}_{1}-1, \ldots, \hat{\lambda}_{k}-1,0, \ldots, 0\right\} T^{-1} \\
& =S T \operatorname{diag}\left\{\hat{\lambda}_{1}-1, \ldots, \hat{\lambda}_{k}-1,0, \ldots, 0\right\} T^{\prime} S .
\end{aligned}
$$

Escrevendo

$$
\hat{\Sigma}-\hat{\Sigma}_{\epsilon}=\left(\begin{array}{ll}
\hat{\Sigma}_{x} & \hat{\Sigma}_{x y} \\
\hat{\Sigma}_{y x} & \hat{\Sigma}_{y}
\end{array}\right)
$$

temos $\hat{\beta} \hat{\Sigma}_{x}=\hat{\Sigma}_{y x}$, ou

$$
\hat{\beta}=\hat{\Sigma}_{y x} \hat{\Sigma}_{x}^{-1}
$$

Para mostrar que a solução é única, devemos verificar a igualdade

$$
\hat{\beta} \hat{\Sigma}_{x} \hat{\beta}^{\prime}=\hat{\Sigma}_{y}
$$

ou ainda, substituindo $\hat{\beta}$ na igualdade acima,

$$
\hat{\Sigma}_{y x} \hat{\Sigma}_{x}^{-1} \hat{\Sigma}_{x y}=\hat{\Sigma}_{y} .
$$

Fazendo

$$
\hat{\Lambda}_{(k)}=\operatorname{diag}\left\{\hat{\lambda}_{1}, \ldots, \hat{\lambda}_{k}\right\}
$$

e particionando $T^{-1}$ em blocos como $T^{-1}=\left(U_{i j}\right), U_{11}: k \times k$, temos, por (4.19)

$$
\left(\begin{array}{ll}
\hat{\Sigma}_{x} & \hat{\Sigma}_{x y} \\
\hat{\Sigma}_{y x} & \hat{\Sigma}_{y}
\end{array}\right)=\left(\begin{array}{ll}
U_{11}\left(\hat{\Lambda}_{(k)}-I\right) U_{11}^{\prime} & U_{11}\left(\hat{\Lambda}_{(k)}-I\right) U_{21}^{\prime} \\
U_{21}\left(\hat{\Lambda}_{(k)}-I\right) U_{11}^{\prime} & U_{21}\left(\hat{\Lambda}_{(k)}-I\right) U_{21}^{\prime}
\end{array}\right),
$$

e isso é o bastante para verificar (4.21).

Tudo isso foi feito com a suposição de que $\hat{\lambda}_{k}>1$. Suponha agora que $\hat{\lambda}_{k} \leq 1$. Lembremos que o nosso problema de maximização reduziu-se a procurar

$$
\max \left\{h_{\hat{\gamma}}\left(\nu_{1}, \ldots, \nu_{k}\right), \nu_{1} \geq \ldots \geq \nu_{k}>1\right\}
$$


que por sua vez é equivalente à procura de

$$
\max \left\{-\frac{1}{2} \eta(\omega, \lambda), \omega>1\right\}
$$

Suponha então que $0<\lambda \leq 1$. A função parcial $\eta_{\lambda}:(1, \infty) \rightarrow \mathbb{R}$ é estritamente crescente, pois

$$
\partial_{1} \eta(\omega, \lambda)=\frac{d n \omega+n \omega d^{2}-d n \lambda-\omega d^{2}}{\omega(n \lambda+d \omega)}
$$

para todo $\omega>1$. Como $d n \omega>d n \lambda$, vem que $\partial_{1} \eta(\omega, \lambda)>0$ para todo $\omega>1$. Portanto,

$$
\lim _{\omega \downarrow 1, \omega>1} \eta(\omega, \lambda)=\eta(1, \lambda)=\inf \{\eta(\omega, \lambda), \omega>1\} .
$$

Seja $q=\#\left\{\hat{\lambda}_{i} ; \hat{\lambda}_{i}>1\right\}$. Então

$$
\begin{aligned}
\sup \left\{G\left(\mu, \Sigma_{T}, \Omega_{T}\right) ;\left(\mu, \Sigma_{T}, \Omega_{T}\right) \in \mathbb{R}^{p} \times \Phi\right\} \\
=-\frac{n+d}{2}\left(p+\sum_{i=k+1}^{p} \log \hat{\gamma}_{i}\right)-\frac{1}{2}\left[\sum_{i=1}^{q} \eta\left(\hat{\lambda}_{i}, \hat{\lambda}_{i}\right)+\sum_{i=q+1}^{k} \eta\left(1, \hat{\lambda}_{i}\right)\right] \\
=-\frac{n+d}{2}\left(p+\sum_{i=k+1}^{p} \log \hat{\gamma}_{i}\right)-\frac{1}{2}\left[\sum_{i=1}^{q} n \log \hat{\lambda}_{i}+\sum_{i=q+1}^{k}(n+d) \log \left(\frac{n \hat{\lambda}_{i}+d}{n+d}\right)\right] \\
=-\frac{n+d}{2}\left[p+\sum_{i=k+1}^{p} \log \hat{\gamma}_{i}+\sum_{i=q+1}^{k} \log \left(\frac{n \hat{\lambda}_{i}+d}{n+d}\right)\right]-\frac{n}{2}\left(\sum_{i=1}^{q} \log \hat{\lambda}_{i}\right),
\end{aligned}
$$

e esse supremo não é atingido em $\mathbb{R}^{p} \times \Phi$. Ele é atingido em um ponto com $\nu_{q+1}=$ $\ldots=\nu_{k}=1$, que não está no espaço paramétrico. Com efeito, esse ponto corresponde a $\hat{\Sigma}_{T}-\hat{\Omega}_{T}=\operatorname{diag}\left\{\hat{\lambda}_{1}-1, \ldots, \hat{\lambda}_{q}-1,0, \ldots, 0\right\}$ e teríamos posto $\left(\hat{\Sigma}_{T}-\hat{\Omega}_{T}\right)=q<k$. Este problema não é grave, pois provaremos na seção 4.3 - ver (4.38) - que, para $\theta \in \Theta^{*}, \hat{\lambda}_{k}>1$ com probabilidade $P_{\theta}$ tendendo a 1 . Mostraremos que $\{\hat{\theta}\}$ é uma seqüência consistente e assintoticamente normal. Esta será então a nossa solução de máxima verossimilhança, sintetizada no enunciado do teorema 4.1.2.

Teorema 4.1.2 Considere o MRME e a verossimilhança associada baseada nas observações $\left\{Z_{i}, i=1 \ldots, n\right\}$ e $S_{d}$. Sejam $\hat{\lambda}_{1} \geq \ldots \geq \hat{\lambda}_{p}$ os autovalores de $S_{Z}$ na métrica $S_{d}$ e $T$ a matriz de autovetores associados. Se $\hat{\lambda}_{k}>1$, então a verossimi- 
lhança é maximizada em $\hat{\theta}=\left(\hat{\mu}_{x}, \hat{\alpha}, \hat{\beta}, \hat{\Sigma}, \hat{\Sigma}_{\epsilon}\right)$ dado por

$$
\begin{aligned}
\hat{\mu}_{x} & =\bar{X}, \hat{\alpha}=\bar{Y}-\hat{\beta} \bar{X}, \hat{\beta}=\hat{\Sigma}_{y x} \hat{\Sigma}_{x}^{-1} \\
\left(\begin{array}{cc}
\hat{\Sigma}_{x} & \hat{\Sigma}_{x y} \\
\hat{\Sigma}_{y x} & \hat{\Sigma}_{y}
\end{array}\right) & =S_{d} T \hat{\Sigma}_{T z} T^{\prime} S_{d}, \\
\hat{\Sigma}_{T z} & =\operatorname{diag}\left\{\hat{\lambda}_{1}-1, \ldots, \hat{\lambda}_{k}-1,0, \ldots, 0\right\} \\
\hat{\Sigma}_{\epsilon} & =S_{d} T \hat{\Sigma}_{T \epsilon} T^{\prime} S_{d}, \\
\hat{\Sigma}_{T \epsilon} & =\operatorname{diag}\left\{1, \ldots, 1, \hat{\sigma}_{T \epsilon, k+1}, \ldots, \hat{\sigma}_{T \epsilon, p}\right\} \\
\hat{\sigma}_{T \epsilon, i} & =\frac{n \hat{\lambda}_{i}+d}{n+d}, i=k+1, \ldots, p .
\end{aligned}
$$

\subsection{Um Teste para a Qualidade do Ajuste}

Nesta seção obteremos um teste de qualidade do ajuste para o MRME. Mais especificamente, sejam $\left\{Z_{i}, i=1, \ldots, n\right\} \sim N I I D(\mu, \Sigma)$ e $d S \sim W\left(d, \Sigma_{\epsilon}\right)$ independentes. Seja $G$ a log-verossimilhança baseada nestas observações. Obteremos a expressão e a distribuição nula assintótica para a estatística do teste da razão de verossimilhança para as hipóteses

$$
\begin{aligned}
& H:\left(\mu, \Sigma, \Sigma_{\epsilon}\right)=\left(\mu(\theta), \Sigma(\theta), \Sigma_{\epsilon}\right), \quad \theta \in \Theta^{*}, \\
& K:\left(\mu, \Sigma, \Sigma_{\epsilon}\right) \in \mathbb{R}^{p} \times \mathcal{M}_{p} \times \mathcal{M}_{p},
\end{aligned}
$$

onde $\mu(\theta)$ e $\Sigma(\theta)$ são definidos em (4.6) e (4.7), respectivamente.

Seja

$$
\begin{aligned}
\Theta= & \left\{\left(\mu_{x}, \alpha, \beta, \Sigma_{x}, D, \Sigma_{\epsilon}\right) ;\left(\mu_{x}, \alpha\right) \in \mathbb{R}^{k} \times \mathbb{R}^{r}, \beta: r \times k, \Sigma_{x} \in \mathcal{M}_{k},\right. \\
& \left.D: r \times r \text { é simétrica }, \Sigma_{\epsilon} \in \mathcal{M}_{p},\left(\begin{array}{ll}
\Sigma_{x} & \Sigma_{x} \beta^{\prime} \\
\beta \Sigma_{x} & \beta \Sigma_{x} \beta^{\prime}+D
\end{array}\right)+\Sigma_{\epsilon} \in \mathcal{M}_{p}\right\} .
\end{aligned}
$$

Observe que podemos redefinir o espaço paramétrico $\Theta^{*}$ como

$$
\Theta^{*}=\{\theta \in \Theta ; D=0\} \text {. }
$$

Particione $\Sigma=\left(\Sigma_{i j}\right), \Sigma_{\epsilon}=\left(\Sigma_{\epsilon i j}\right), i, j=1,2, \Sigma_{11}, \Sigma_{\epsilon 11}: k \times k$, defina o conjunto

$$
\Xi=\left\{\left(\Sigma, \Sigma_{\epsilon}\right) \in \mathcal{M}_{p} \times \mathcal{M}_{p} ; \Sigma_{11}-\Sigma_{\epsilon 11} \in \mathcal{M}_{k}\right\}
$$


e a aplicação $\psi: \Theta \rightarrow \mathbb{R}^{p} \times \Xi$ dada por

$$
\psi\left(\mu, \alpha, \beta, \Sigma_{x}, D, \Sigma_{\epsilon}\right)=\left[\left(\begin{array}{c}
\mu_{x} \\
\alpha+\beta \mu_{x}
\end{array}\right),\left(\begin{array}{ll}
\Sigma_{x} & \Sigma_{x} \beta^{\prime} \\
\beta \Sigma_{x} & \beta \Sigma_{x} \beta^{\prime}+D
\end{array}\right)+\Sigma_{\epsilon}, \Sigma_{\epsilon}\right]
$$

Temos que $\psi$ é sobrejetiva. Para ver isto, tome $\phi=\left(\left(\mu_{1}^{\prime}, \mu_{2}^{\prime}\right)^{\prime}, \Sigma, \Sigma_{\epsilon}\right) \in \Xi$, com $\mu_{1}: k \times 1$; a igualdade $\psi\left(\mu_{x}, \alpha, \beta, \Sigma_{x}, D, \Sigma_{\epsilon}\right)=\phi$ implica em $\Sigma_{x}=\Sigma_{11}-\Sigma_{\epsilon 11}$, $\mu_{x}=\mu_{1}$,

$$
\begin{aligned}
\beta & =\left(\Sigma_{21}-\Sigma_{\epsilon 21}\right)\left(\Sigma_{11}-\Sigma_{\epsilon 11}\right)^{-1} \\
\alpha & =\mu_{2}-\left(\Sigma_{12}-\Sigma_{\epsilon 21}\right)\left(\Sigma_{11}-\Sigma_{\epsilon 11}\right)^{-1} \mu_{1} \\
D & =\Sigma_{22}-\beta \Sigma_{x} \beta^{\prime}-\Sigma_{\epsilon 22}
\end{aligned}
$$

Provamos facilmente que $\psi$ também é injetiva. Assim, $\psi$ é uma bijeção entre $\Theta$ e $\mathbb{R}^{p} \times \Xi$.

No que segue vamos supor que $d^{-1}=O\left(n^{-1}\right)$. Temos que $G$ é maximizada em $\left(\bar{Z}, S_{Z}, S\right)$. Seja $\phi=\left(\mu, \Sigma, \Sigma_{\epsilon}\right) \in \mathbb{R}^{p} \times \Xi$. Particionando $S=\left(S_{i j}\right), S_{Z}=\left(S_{Z i j}\right)$, $i, j=1,2, S_{11}, S_{Z 11}: k \times k$, temos

$$
S_{Z 11}-S_{11} \stackrel{\text { qc }(\phi)}{\longrightarrow} \Sigma_{11}-\Sigma_{\epsilon 11}>0, \text { quando } n \rightarrow \infty
$$

Assim, com probabilidade $P_{\phi}$ igual a $1,\left(\bar{Z}, S_{Z}, S\right)$ maximiza $\left.G\right|_{\mathbb{R}^{p} \Xi}$, para $n$ e $d$ suficientemente grandes. Isto implica em

$$
\lim _{n \rightarrow \infty} P_{\phi}\left(\sup G=\left.\sup G\right|_{\mathbb{R}^{p} \times}\right)=1 .
$$

Sejam $\phi \in \psi\left(\Theta^{*}\right) \subset \mathbb{R}^{p} \times \Xi=\psi(\Theta)$ e $\zeta$ a estatística da razão de verossimilhança para o teste de $H$ contra $K$. Então,

$$
\begin{aligned}
P_{\phi}\left(-2 \log \zeta \geq c_{0}\right)= & P_{\phi}\left[-2\left(\left.\sup G\right|_{\psi\left(\Theta^{*}\right)}-\sup G\right) \geq c_{0}\right] \\
= & P_{\phi}\left[2\left(\sup G-\left.\sup G\right|_{\psi\left(\Theta^{*}\right)}\right) \geq c_{0}, \sup G=\left.\sup G\right|_{\psi(\Theta)}\right] \\
& +P_{\phi}\left[2\left(\sup G-\left.\sup G\right|_{\psi\left(\Theta^{*}\right)}\right) \geq c_{0}, \sup G \neq\left.\sup G\right|_{\psi(\Theta)}\right]
\end{aligned}
$$

e, por (4.25), $P_{\phi}\left(-2 \log \zeta \geq c_{0}\right)$ terá o mesmo limite (quando $\left.n \rightarrow \infty\right)$ de $P_{\phi}\left[2\left(\left.\sup G\right|_{\psi(\Theta)}\right.\right.$ $\left.\left.-\left.\sup G\right|_{\psi\left(\Theta^{*}\right)}\right) \geq c_{0}\right]$. Assim, pela teoria assintótica usual para o teste de razão de verossimilhança (ver Wilks, 1962, pg. 419) temos que, quando $H$ é verdadeira, $-2 \log \zeta$ tem distribuição limite $\chi_{g l}^{2}$, onde $g l=(1 / 2) r(r+1)$ é o número de parâmetros fixados sob $H$, pois, sob a hipótese nula, a matriz $D$ é nula (ver 4.24). 
Vamos obter a expressão para a estatística de teste baseada no estimador apresentado no Teorema 4.1.2. Primeiramente, temos

$$
\sup G=G\left(\bar{Z}, T^{\prime} S_{Z} T, T^{\prime} S T\right)=G\left(\bar{Z} \cdot \hat{\Lambda}, I_{p}\right)
$$

Assim,

$$
\begin{aligned}
\sup G & =-\frac{n}{2}\left(\log |\hat{\Lambda}|+\operatorname{tr}\left(I_{p}\right)\right)-\frac{d}{2}\left(\log \left|I_{p}\right|+\operatorname{tr}\left(I_{p}\right)\right) \\
& =-\frac{n}{2}\left(\sum_{i=1}^{p} \log \hat{\lambda}_{i}+p\right)-\frac{d p}{2}
\end{aligned}
$$

Pelo desenvolvimento que levou à demonstração do Teorema 4.1.2, temos que o supremo no numerador de $\zeta$ é igual ao supremo da verossimilhança no conjunto $\Phi$, dado em (4.12). Por (4.23) temos que

$$
\sup \left\{\left.G\right|_{\psi\left(\Theta^{*}\right)}\right\}=-\frac{n+d}{2}\left(p+\sum_{i=s+1}^{p} \log \hat{\sigma}_{T \epsilon i}\right)-\frac{n}{2}\left(\sum_{i=1}^{s} \log \hat{\lambda}_{i}\right),
$$

onde $s=\min \{q, k\}, q=\#\left\{\hat{\lambda}_{i} ; \hat{\lambda}_{i}>1\right\}$, e

$$
\hat{\sigma}_{T \epsilon i}=(n+d)^{-1}\left(n \hat{\lambda}_{i}+d\right), i=s+1, \ldots, p .
$$

Por (4.26) e (4.27) resulta que

$$
-2 \log \zeta=n \sum_{i=s+1}^{p} \log \left(\hat{\sigma}_{T \epsilon i}-\log \hat{\lambda}_{i}\right)+d \sum_{i=s+1}^{p} \log \hat{\sigma}_{T \epsilon i} .
$$

O teste de qualidade do ajuste para o MRME é descrito no teorema 4.2.1.

Teorema 4.2.1 O teste da razão de verossimilhança, de nível assintótico $\xi$, de qualidade do ajuste para o MRME, ou seja, para testar

$$
H:\left(\mu, \Sigma, \Sigma_{\epsilon}\right)=\left(\mu(\theta), \Sigma(\theta), \Sigma_{\epsilon}\right), \theta \in \Theta^{*}
$$

contra

$$
K:\left(\mu, \Sigma, \Sigma_{\epsilon}\right) \in \mathbb{R}^{p} \times \mathcal{M}_{p} \times \mathcal{M}_{p}
$$

é dado por: rejeitar $H$ se e somente se $-2 \log \zeta \geq c_{\xi}$, onde $\zeta$ é dado em (4.28) e $c_{\xi}$ é tal que $P\left(\chi_{\frac{1}{2} r(r+1)}^{2} \geq c_{\xi}\right)=\xi$. 


\subsection{Consistência}

Para provar que os estimadores definidos no Teorema 4.1.2 são fortemente consistentes não precisamos da hipótese de normalidade dada em (4.3). Esta suposição será substituída por

1. $\left\{x_{i}, i \in \mathbb{N}\right\}$ e $\left\{\epsilon_{i}, i \in \mathbb{N}\right\}$ são independentes;

2. $\left\{\left(x_{i}^{\prime}, \epsilon_{i}^{\prime}\right)^{\prime}, i \in \mathbb{N}\right\} \sim \operatorname{IID}\left[\left(\mu_{x}^{\prime}, 0\right)^{\prime}, \operatorname{block} \operatorname{diag}\left\{\Sigma_{x}, \Sigma_{\epsilon}\right\}\right], \operatorname{com} \Sigma_{x}>0, \Sigma_{\epsilon}>0$.

O Teorema 4.3.1 é o principal resultado desta seção. Na sua demonstração usaremos os seguintes resultados básicos: seja $\lambda$ autovalor de uma matriz $A: n \times n$, com multiplicidade algébrica $n(\lambda)$; seja $U: n \times n(\lambda)$ uma matriz cujas colunas são autovetores ortogonais associados à $\lambda$. Então qualquer matriz de ordem $n \times n(\lambda)$ formada por autovetores ortogonais associados à $\lambda$ nas colunas é da forma $U G$, onde $G: n(\lambda) \times n(\lambda)$ é ortogonal. Também, se $\left\{b_{n}\right\}$ é uma sequência no espaço Euclideano, temos que $b_{n} \rightarrow b$ se e somente se toda subsequência de $\left\{b_{n}\right\}$ possuir uma subsubsequência que converge para $b$.

Será crucial a utilização do lema 4.3.1, cuja demonstração pode ser encontrada em Franklin (1968, pg. 191).

Lema 4.3.1 Seja $A=\left(A_{i j}\right)$ uma matriz de ordem $m \times m$. Seja $\lambda_{i}$ autovalor de $A$ com multiplicidade algébrica $n_{i}, i=1, \ldots, s$. Então, dado $\eta>0$ existe $\xi>0$ tal que, se $\left|B_{i j}-A_{i j}\right|<\xi$ para $i, j=1, \ldots, m$, a matriz $B=\left(B_{i j}\right)$ tem exatamente $n_{i}$ autovalores na bola aberta de centro $\lambda_{i}$ e raio $\eta$ para cada $i=1, \ldots, s$.

Teorema 4.3.1 Considere o modelo dado por (4.1), (4.2), (1) e (2). Seja $d S \sim$ $W\left(d, \Sigma_{\epsilon}\right)$, onde $d^{-1}=O\left(n^{-1}\right)$. Então, para todo $\theta \in \Theta^{*}$,

$$
\left(\hat{\mu}_{x}, \hat{\alpha}, \hat{\beta}, \hat{\Sigma}_{x}, \hat{\Sigma}_{\epsilon}\right) \stackrel{q c(\theta)}{\longrightarrow}\left(\mu_{x}, \alpha, \beta, \Sigma_{x}, \Sigma_{\epsilon}\right), \text { quando } n \rightarrow \infty .
$$

Demonstração: As convergências a seguir ocorrem quando $n \rightarrow \infty$. Seja $\theta \in \Theta^{*}$; primeiramente provaremos que

$$
\hat{\Sigma}_{z} \stackrel{\mathrm{qc}(\theta)}{\longrightarrow} \Sigma_{z}(\theta)
$$

onde $\hat{\Sigma}_{z}=\left(T^{\prime}\right)^{-1} \hat{\Sigma}_{T z} T^{\prime}$ e $\Sigma_{z}$ está definido em (4.8). A consistência dos estimadores será uma consequência direta desse resultado. Seja

$$
\hat{Q}=\left(\hat{Q}_{(k)}, \hat{Q}_{(r)}\right),
$$


$\hat{Q}_{(k)}: p \times k$, uma matriz ortogonal de autovetores de $S^{-1 / 2} S_{Z} S^{-1 / 2}$ associados a $\hat{\Lambda}$ e faça $\hat{\Lambda}=$ block $\operatorname{diag}\left\{\hat{\Lambda}_{(k)}, \hat{\Lambda}_{(r)}\right\}$, onde $\hat{\Lambda}_{(k)}: k \times k$ e $\hat{\Lambda}$ é definida em (4.15). Por (4.11) e (4.19) temos que

$$
\hat{\Sigma}_{z}=S^{1 / 2} \hat{Q}_{(k)}\left(\hat{\Lambda}_{(k)}-I\right) \hat{Q}_{(k)}^{\prime} S^{1 / 2} .
$$

Seja $\Sigma=\Sigma(\theta)$ definida em (4.7). Pela Lei Forte dos Grandes Números,

$$
S_{Z} \stackrel{\mathrm{qc}(\theta)}{\longrightarrow} \Sigma
$$

pelo Teorema 3.3.2 de Anderson (1984), pela Lei Forte dos Grandes Números, e observando que $\lim _{n \rightarrow \infty} d=\infty$, vem que

$$
S \stackrel{\mathrm{qc}(\theta)}{\longrightarrow} \Sigma_{\epsilon}
$$

Sejam

$$
\begin{aligned}
& \Sigma_{\rho}=\left[\left(I, \beta^{\prime}\right) \Sigma_{\epsilon}^{-1}\left(I, \beta^{\prime}\right)^{\prime}\right]^{-1} \\
& \Sigma_{\nu}=(-\beta, I) \Sigma_{\epsilon}(-\beta, I)^{\prime}
\end{aligned}
$$

e

$$
\Lambda=\text { block } \operatorname{diag}\left\{\Lambda_{(k)}, \Lambda_{(r)}\right\},
$$

onde $\Lambda_{(k)}=\operatorname{diag}\left\{\lambda_{1}, \ldots, \lambda_{k}\right\}, \Lambda_{(r)}=\operatorname{diag}\left\{\lambda_{k+1}, \ldots, \lambda_{p}\right\}$, e $\lambda_{1} \geq \ldots \geq \lambda_{p}$ são os autovalores de $\Sigma_{\epsilon}^{-1 / 2} \Sigma \Sigma_{\epsilon}^{-1 / 2}=\Sigma_{\epsilon}^{-1 / 2}\left(I, \beta^{\prime}\right)^{\prime} \Sigma_{x}\left(I, \beta^{\prime}\right) \Sigma_{\epsilon}^{-1 / 2}+I$. Temos que $\lambda_{i}=1+\nu_{i}$, onde $\nu_{1} \geq \ldots \geq \nu_{p}$ são os autovalores de $\Sigma_{\epsilon}^{-1 / 2}\left(I, \beta^{\prime}\right)^{\prime} \Sigma_{x}\left(I, \beta^{\prime}\right) \Sigma_{\epsilon}^{-1 / 2}$. Pelo Teorema A.6.2 de Mardia et al. (1979), os autovalores não nulos — positivos, neste caso desta matriz são os mesmos de

$$
\Sigma_{x}^{1 / 2}\left(I, \beta^{\prime}\right) \Sigma_{\epsilon}^{-1}\left(I, \beta^{\prime}\right)^{\prime} \Sigma_{x}^{1 / 2}=\Sigma_{x}^{1 / 2} \Sigma_{\rho}^{-1} \Sigma_{x}^{1 / 2}
$$

e, pelo mesmo resultado, também são autovalores de

$$
\Sigma_{\rho}^{-1 / 2} \Sigma_{x}^{1 / 2} \Sigma_{x}^{1 / 2} \Sigma_{\rho}^{-1 / 2}=\Sigma_{\rho}^{-1 / 2} \Sigma_{x} \Sigma_{\rho}^{-1 / 2}
$$

Assim, $\lambda_{i}=1+\nu_{i}$, onde $\nu_{1} \geq \ldots \geq \nu_{k}>0$ são os autovalores de $\Sigma_{\rho}^{-1 / 2} \Sigma_{x} \Sigma_{\rho}^{-1 / 2}$, e $\lambda_{i}=1, i=k+1, \ldots, p$. Ou seja, $\Lambda_{(r)}=I$ e a matriz de autovalores de $\Sigma_{\rho}^{-1 / 2} \Sigma_{x} \Sigma_{\rho}^{-1 / 2}$ é $\Lambda_{(k)}-I$. Seja $R$ a matriz ortogonal de autovetores correspondente. Então

$$
Q_{(k)}=\Sigma_{\epsilon}^{-1 / 2}\left(I, \beta^{\prime}\right)^{\prime} \Sigma_{\rho}^{1 / 2} R,
$$


é uma matriz ortogonal de autovetores de $\Sigma_{\epsilon}^{-1 / 2} \Sigma \Sigma_{\epsilon}^{-1 / 2}$ correspondente a $\Lambda_{(k)}$. Com efeito,

$$
\begin{aligned}
& Q_{(k)}^{\prime} Q_{(k)}=R^{\prime} \Sigma_{\rho}^{1 / 2}\left(I, \beta^{\prime}\right) \Sigma_{\epsilon}^{-1 / 2} \Sigma_{\epsilon}^{-1 / 2}\left(I, \beta^{\prime}\right)^{\prime} \Sigma_{\rho}^{1 / 2} R=I \\
& \Sigma_{\epsilon}^{-1 / 2} \Sigma \Sigma_{\epsilon}^{-1 / 2} Q_{(k)}=\Sigma_{\epsilon}^{-1 / 2}\left[\left(I, \beta^{\prime}\right)^{\prime} \Sigma_{x}\left(I, \beta^{\prime}\right)\right] \Sigma_{\epsilon}^{-1 / 2} Q_{(k)}+Q_{(k)} \\
&=\Sigma_{\epsilon}^{-1 / 2}\left[\left(I, \beta^{\prime}\right)^{\prime} \Sigma_{x}\left(I, \beta^{\prime}\right) \Sigma_{\epsilon}^{-1 / 2} \Sigma_{\epsilon}^{-1 / 2}\left(I, \beta^{\prime}\right)^{\prime} \Sigma_{\rho}^{1 / 2} R\right]+\Sigma_{\epsilon}^{-1 / 2}\left(I, \beta^{\prime}\right)^{\prime} \Sigma_{\rho}^{1 / 2} R \\
&=\Sigma_{\epsilon}^{-1 / 2}\left[\left(I, \beta^{\prime}\right)^{\prime} \Sigma_{x} \Sigma_{\rho}^{-1 / 2} R\right]+\Sigma_{\epsilon}^{-1 / 2}\left[\left(I, \beta^{\prime}\right)^{\prime} \Sigma_{\rho}^{1 / 2} R\right] \\
&=\Sigma_{\epsilon}^{-1 / 2}\left[\left(I, \beta^{\prime}\right)^{\prime} \Sigma_{\rho}^{1 / 2} R\left(\Lambda_{(k)}-I\right)\right]+\Sigma_{\epsilon}^{-1 / 2}\left[\left(I, \beta^{\prime}\right)^{\prime} \Sigma_{\rho}^{1 / 2} R\right] \\
&=\Sigma_{\epsilon}^{-1 / 2}\left(I, \beta^{\prime}\right)^{\prime} \Sigma_{\rho}^{1 / 2} R\left(\Lambda_{(k)}-I+I\right) \\
&=\Sigma_{\epsilon}^{-1 / 2}\left(I, \beta^{\prime}\right)^{\prime} \Sigma_{\rho}^{1 / 2} R \Lambda_{(k)}=Q_{(k)} \Lambda_{(k)} .
\end{aligned}
$$

A quarta igualdade vale pela definição de $R$. Analogamente provamos que

$$
Q_{(r)}=\Sigma_{\epsilon}^{1 / 2}(-\beta, I)^{\prime} \Sigma_{\nu}^{-1 / 2}
$$

é uma matriz ortogonal de autovetores de $\Sigma_{\epsilon}^{-1 / 2} \Sigma \Sigma_{\epsilon}^{-1 / 2}$ associada aos autovalores iguais a 1 . Assim, uma matriz de autovetores de $\Sigma_{\epsilon}^{-1 / 2} \Sigma \Sigma_{\epsilon}^{-1 / 2}$ correspondente a $\Lambda$ é da forma

$$
\left(Q_{(k)} G, Q_{(r)} H\right)
$$

onde

$$
G=\text { block } \operatorname{diag}\left\{G_{1}, \ldots, G_{s}\right\}
$$

$G_{i}, i=1, \ldots, s$ e $H$ são matrizes ortogonais e $s$ é o número de autovalores distintos $\lambda_{i}$.

Pelo lema 4.3.1, a função que associa a cada matriz simétrica os seus autovalores ordenados é contínua. Assim, por (4.31) e (4.32) temos que

$$
\hat{\Lambda} \stackrel{\mathrm{qc}(\theta)}{\longrightarrow} \Lambda \text {. }
$$

Seja $\omega$ um ponto no espaço de probabilidades definido pela amostra, satisfazendo (4.31), (4.32) e (4.38). O conjunto formado por tais $\omega$ tem probabilidade $P_{\theta}$ igual a 1. Por ser formada por matrizes ortogonais, $\{\hat{Q}(\omega)\}$ é uma sequência limitada de $\mathbb{R}^{p^{2}}$. Assim, toda subsequência de $\{\hat{Q}(\omega)\}$ possui uma subsubsequência convergente. Sem 
risco de confusão, usaremos $\{\hat{Q}(\omega)\}$ para denotar esta subsubsequência. Tomando limites em

$$
S^{-1 / 2}(\omega) S_{Z}(\omega) S^{-1 / 2}(\omega) \hat{Q}(\omega)=\hat{Q}(\omega) \hat{\Lambda}(\omega)
$$

$\mathrm{e}$

$$
\hat{Q}^{\prime}(\omega) Q(\omega)=I
$$

vemos que $\{\hat{Q}(\omega)\}$ converge para uma matriz ortogonal formada por autovetores de $\Sigma_{\epsilon}^{-1 / 2} \Sigma \Sigma_{\epsilon}^{-1 / 2}$ associada a $\Lambda$. O limite da subsubsequência é então da forma dada em (4.36), para algumas matrizes ortogonais $G(\omega)$ e $H(\omega)$. Assim, o limite de $\hat{Q}_{(k)}(\omega) \hat{\Lambda}_{(k)}(\omega) \hat{Q}_{(k)}^{\prime}(\omega)$ é dado por

$$
\begin{aligned}
Q_{(k)} G(\omega) \Lambda_{(k)} G^{\prime}(\omega) Q_{(k)}^{\prime} & =Q_{(k)} \Lambda_{(k)} Q_{(k)}^{\prime} \\
& =\Sigma_{\epsilon}^{-1 / 2}\left(I, \beta^{\prime}\right)^{\prime} \Sigma_{\rho}^{1 / 2} R \Lambda_{(k)} R^{\prime} \Sigma_{\rho}^{1 / 2}\left(I, \beta^{\prime}\right) \Sigma_{\epsilon}^{-1 / 2} \\
& =\Sigma_{\epsilon}^{-1 / 2}\left(I, \beta^{\prime}\right)^{\prime} \Sigma_{\rho}^{1 / 2}\left(\Sigma_{\rho}^{-1 / 2} \Sigma_{x} \Sigma_{\rho}^{-1 / 2}+I\right) \Sigma_{\rho}^{1 / 2}\left(I, \beta^{\prime}\right) \Sigma_{\epsilon}^{-1 / 2} \\
& =\Sigma_{\epsilon}^{-1 / 2}\left(I, \beta^{\prime}\right)^{\prime} \Sigma_{x}\left(I, \beta^{\prime}\right) \Sigma_{\epsilon}^{-1 / 2}+\Sigma_{\epsilon}^{-1 / 2}\left(I, \beta^{\prime}\right)^{\prime} \Sigma_{\rho}\left(I, \beta^{\prime}\right) \Sigma_{\epsilon}^{-1 / 2} \\
& =\Sigma_{\epsilon}^{-1 / 2}\left[\Sigma_{z}+\left(I, \beta^{\prime}\right)^{\prime} \Sigma_{\rho}\left(I, \beta^{\prime}\right)\right] \Sigma_{\epsilon}^{-1 / 2},
\end{aligned}
$$

que não depende da subsubsequência. Voltando à sequência original, concluimos que

$$
\hat{Q}_{(k)} \hat{\Lambda}_{(k)} \hat{Q}_{(k)}^{\prime} \stackrel{\mathrm{qc}(\theta)}{\longrightarrow} \Sigma_{\epsilon}^{-1 / 2}\left(\Sigma_{z}+\left(I, \beta^{\prime}\right)^{\prime} \Sigma_{\rho}\left(I, \beta^{\prime}\right)\right) \Sigma_{\epsilon}^{-1 / 2} .
$$

Analogamente, mostramos que

$$
\hat{Q}_{(k)} \hat{Q}_{(k)}^{\prime} \stackrel{\mathrm{qc}(\theta)}{\longrightarrow} \Sigma_{\epsilon}^{-1 / 2}\left(I, \beta^{\prime}\right)^{\prime} \Sigma_{\rho}\left(I, \beta^{\prime}\right) \Sigma_{\epsilon}^{-1 / 2} .
$$

Por (4.40) e (4.41) temos

$$
\hat{\Sigma}_{z}=S^{1 / 2} \hat{Q}_{(k)}\left(\hat{\Lambda}_{(k)}-I\right) \hat{Q}_{(k)}^{\prime} S^{1 / 2} \stackrel{\mathrm{qc}(\theta)}{\longrightarrow} \Sigma_{z}
$$

provando (4.29). Como

$$
\hat{\Sigma}_{z}=\left(\begin{array}{ll}
\hat{\Sigma}_{x} & \hat{\Sigma}_{x} \hat{\beta}^{\prime} \\
\hat{\beta} \hat{\Sigma}_{x} & \hat{\beta} \hat{\Sigma}_{x} \hat{\beta}^{\prime}
\end{array}\right)
$$

temos a consistência forte de $\hat{\Sigma}_{x}$ e $\hat{\beta}$. Vamos mostrar agora que $\hat{\Sigma}_{\epsilon} \stackrel{\mathrm{qc}(\theta)}{\longrightarrow} \Sigma_{\epsilon}$. Primeiramente, vamos exibir uma expressão alternativa para $\hat{\Sigma}_{\epsilon}$. Temos que

$$
\hat{\Sigma}_{\epsilon}=\left(T^{\prime}\right)^{-1} \text { block } \operatorname{diag}\{I, \mathcal{H}\} T^{-1},
$$


onde

$$
\mathcal{H}=\operatorname{diag}\left\{\frac{n \hat{\lambda}_{k+1}+d}{n+d}, \ldots, \frac{n \hat{\lambda}_{p}+d}{n+d}\right\}=\frac{1}{n+d}\left(n \hat{\Lambda}_{(r)}+d I\right)
$$

Assim,

$$
\begin{aligned}
\hat{\Sigma}_{\epsilon} & =\frac{1}{n+d}\left(T^{\prime}\right)^{-1}\left(\text { block } \operatorname{diag}\left\{(n+d) I, n \hat{\Lambda}_{(r)}+d I\right\}\right) T^{-1} \\
& =\frac{1}{n+d}\left(T^{\prime}\right)^{-1}\left[n\left(\operatorname{block} \operatorname{diag}\left\{I, \hat{\Lambda}_{(r)}\right\}\right)+d I\right] T^{-1} \\
& =\frac{1}{n+d}\left(T^{\prime}\right)^{-1}\left[n\left(\operatorname{block} \operatorname{diag}\left\{\hat{\Lambda}_{(k)}-\hat{\Lambda}_{(k)}+I, \hat{\Lambda}_{(r)}\right\}\right)+d I\right] T^{-1} \\
& =\frac{1}{n+d}\left(T^{\prime}\right)^{-1}\left[n\left(\hat{\Lambda}-\operatorname{block} \operatorname{diag}\left\{\hat{\Lambda}_{(k)}-I, 0\right\}\right)+d I\right] T^{-1} \\
& =\frac{1}{n+d}\left\{n\left(S_{Z}-\hat{\Sigma}_{z}\right)+d S\right\}
\end{aligned}
$$

Temos que

$$
S_{Z}-\hat{\Sigma}_{z} \stackrel{\mathrm{qc}(\theta)}{\longrightarrow} \Sigma-\Sigma_{z}=\Sigma_{\epsilon}
$$

Por (4.42),

$$
\begin{aligned}
\hat{\Sigma}_{\epsilon} & =\frac{1}{n+d}\left[n\left(S_{Z}-\hat{\Sigma}_{z}\right)+d S+n S-n S\right]=\frac{1}{n+d}\left[n\left(S_{Z}-\hat{\Sigma}_{z}-S\right)+(d+n) S\right] \\
& =\frac{n}{n+d}\left(S_{Z}-\hat{\Sigma}_{z}-S\right)+S \stackrel{\mathrm{qc}(\theta)}{\longrightarrow} \Sigma_{\epsilon} .
\end{aligned}
$$

Como $\hat{\mu}_{x}=\bar{X}$, vem que $\hat{\mu}_{x} \stackrel{\mathrm{qc}(\theta)}{\longrightarrow} \mu_{x}$. Como $\hat{\alpha}=\bar{Y}-\hat{\beta} \bar{X}$, vem que $\hat{\alpha} \stackrel{\mathrm{qc}(\theta)}{\longrightarrow} \alpha$, pois

$$
Y_{i}=y_{i}+e_{i} \Longrightarrow \bar{Y} \stackrel{\mathrm{qc}(\theta)}{\longrightarrow} E\left(Y_{i}\right)=\alpha+\beta \mu_{x} \text {. }
$$

\subsection{Normalidade Assintótica}

Nesta seção encontramos a distribuição assintótica da sequência de vetores $\left\{n^{1 / 2} \operatorname{vec}(\hat{\beta}-\right.$ $\beta)$ \}. Para isso vamos considerar um modelo definido por (4.1), (4.2), com as seguintes suposições:

1. $\left\{\epsilon_{i}, i \in \mathbb{N}\right\} \sim N I I D\left(0, \Sigma_{\epsilon}\right)$ e $\left\{x_{i}, i \in \mathbb{N}\right\} \sim I I D\left(\mu_{x}, \Sigma_{x}\right)$ são independentes, $\operatorname{com} \Sigma_{x}>0, \Sigma_{\epsilon}>0$;

2. Existe uma sequência consistente de estimadores de $\Sigma_{\epsilon},\left\{S_{d}\right\}$, independente de $\left\{Z_{i}, i \in \mathbb{N}\right\}$, tal que $d S_{d} \sim W\left(d, \Sigma_{\epsilon}\right)$ 
3. $d^{-1}=O\left(n^{-1}\right), \lim _{n \rightarrow \infty} \frac{n}{d}=c, 0<c<\infty$.

Usaremos definições e propriedades dos operadores vec e vech, bem como suas relações com o produto de Kronecker $\otimes$. Este material é detalhado em Henderson \& Searle (1979). As definições e propriedades utilizadas aqui são enunciadas a seguir.

Sejam $a_{i}: s \times 1, i=1, \ldots, t$ as colunas da matriz $A: s \times t$. Então

$$
\operatorname{vec}(A)=\left(\begin{array}{c}
a_{1} \\
a_{2} \\
\vdots \\
a_{t}
\end{array}\right) .
$$

Para uma matriz quadrada $A$, definimos vech $(A)$ da mesma maneira que $\operatorname{vec}(A)$, exceto que para cada coluna de $A$ somente aquela parte que pertence ou está abaixo da diagonal principal de $A$ é usada para construir vech $(A)$.

Definimos uma matriz de vec-permutação como sendo $I_{(s, t)}$ tal que

$$
\operatorname{vec}(A)=I_{(s, t)} \operatorname{vec}\left(A^{\prime}\right)
$$

Se $A=\left(a_{i j}\right)$ e $B$ são matrizes de ordem quaisquer, o produto de Kronecker é definido por

$$
A \otimes B=\left(a_{i j} B\right) .
$$

Para $A, B$ e $C$ de dimensões convenientes,

$$
\begin{aligned}
(A \otimes B)(C \otimes D) & =A C \otimes B D \\
(A \otimes B)^{\prime} & =A^{\prime} \otimes B^{\prime} ; \\
(A \otimes B)^{-1} & =A^{-1} \otimes B^{-1} ; \\
\operatorname{vec}(A B C) & =\left(C^{\prime} \otimes A\right) \operatorname{vec}(B) ; \\
\operatorname{tr}\left(A^{\prime} B\right) & =(\operatorname{vec}(A))^{\prime} \operatorname{vec}(B) .
\end{aligned}
$$

Matrizes de vec-permutação satisfazem

$$
I_{(s, t)}^{\prime}=I_{(t, s)}, \quad I_{(s, t)} I_{(t, s)}=I_{s t} .
$$

Se $A: s \times t$ e $B: u \times v$ então,

$$
B \otimes A=I_{(s, u)}(A \otimes B) I_{(v, t)} .
$$


Se $A, B: t \times t$,

$$
I_{(t, t)}(B \otimes A)=(A \otimes B) I_{(t, t)} .
$$

Existe uma matriz $\Upsilon_{v}: p^{2} \times \frac{1}{2} v(v+1)$ tal que, para toda matriz $A: v \times v$ simétrica,

$$
\operatorname{vec}(A)=\Upsilon_{v} \operatorname{vech}(A)
$$

Seja $\Delta_{v}=\left(\Upsilon_{v}^{\prime} \Upsilon_{v}\right)^{-1} \Upsilon_{v}^{\prime}$. Então,

$$
\begin{aligned}
\operatorname{vech}(A) & =\Delta_{v} \operatorname{vec}(A) \\
\Upsilon_{v} \Delta_{v} & =\frac{1}{2}\left(I_{v^{2}}+I_{(v, v)}\right) \\
\Delta_{v} I_{(v, v)} & =\Delta_{v} .
\end{aligned}
$$

Para uma matriz quadrada $A$,

$$
\begin{aligned}
\Upsilon_{v} \Delta_{v}(A \otimes A) & =(A \otimes A) \Delta_{v} \Upsilon_{v} \\
\Delta_{v}(A \otimes A) & =\Delta_{v}(A \otimes A) \Upsilon_{v} \Delta_{v}
\end{aligned}
$$

Vamos encontrar a distribuição assintótica. Tome $\theta \in \Theta^{*}$. Defina a partição

$$
T=\left(T_{(k)}, T_{(r)}\right)=\left(\begin{array}{cc}
T_{k k} & T_{k r} \\
T_{r k} & T_{r r}
\end{array}\right),
$$

$T_{k k}: k \times k$. Seja

$$
P_{(k)}=S^{1 / 2} \hat{Q}_{(k)}=S T_{(k)}
$$

— veja a relação entre $T$ e $\hat{Q}$ em $(4.11)$ - e façamos $P_{(k)}=\left(P_{k k}^{\prime}, P_{r k}^{\prime}\right)^{\prime}, \operatorname{com} P_{k k}$ : $k \times k$. Tomemos $\omega$ e uma subsubsequência $\{\hat{Q}(\omega)\}$ como na demonstração do Teorema 4.3.1. Mostramos que o limite de $\hat{Q}_{(r)}(\omega)$ é $Q_{(r)} H(\omega)=\Sigma_{\epsilon}^{1 / 2}(-\beta, I)^{\prime} \Sigma_{\nu}^{-1 / 2} H(\omega)$, com $H(\omega)$ ortogonal. Assim, $T_{(r)}(\omega)=S^{-1 / 2}(\omega) \hat{Q}_{(r)}(\omega)$ converge para

$$
(-\beta, I)^{\prime} \Sigma_{\nu}^{-1 / 2} H(\omega)=\left(\begin{array}{c}
-\beta^{\prime} \Sigma_{\nu}^{-1 / 2} H(\omega) \\
\Sigma_{\nu}^{-1 / 2} H(\omega)
\end{array}\right) .
$$

De forma análoga podemos provar que $P_{(k)}(\omega)$ converge para

$$
\left(I, \beta^{\prime}\right)^{\prime} \Sigma_{\rho}^{1 / 2} R G(\omega)=\left(\begin{array}{c}
\Sigma_{\rho}^{1 / 2} R G(\omega) \\
\beta \Sigma_{\rho}^{1 / 2} R G(\omega)
\end{array}\right)
$$


com $G$ definida em (4.37). Assim, usando (4.38), temos

$$
\begin{aligned}
\lim _{n \rightarrow \infty} T_{r r}(\omega) \hat{\Lambda}_{(r)}(\omega) T_{r r}^{\prime}(\omega) & =\Sigma_{\nu}^{-1}, \\
\lim _{n \rightarrow \infty} T_{r r}(\omega) T_{r r}^{\prime}(\omega) & =\Sigma_{\nu}^{-1},
\end{aligned}
$$

e

$$
\lim _{n \rightarrow \infty} P_{k k}(\omega) \hat{\Lambda}_{(k)}(\omega) P_{k k}^{\prime}(\omega)=\Sigma_{\rho}^{1 / 2} R \Lambda_{(k)} R^{\prime} \Sigma_{\rho}^{1 / 2}=\Sigma_{x}+\Sigma_{\rho}
$$

a última igualdade valendo pela definição de $R$. Ou seja,

$$
\begin{aligned}
T_{r r} \hat{\Lambda}_{(r)} T_{r r}^{\prime} & \stackrel{\mathrm{qc}(\theta)}{\longrightarrow} \Sigma_{\nu}^{-1}, \\
T_{r r} T_{r r}^{\prime} & \stackrel{\mathrm{qc}(\theta)}{\longrightarrow} \Sigma_{\nu}^{-1}
\end{aligned}
$$

e

$$
P_{k k} \hat{\Lambda}_{(k)} P_{k k}^{\prime} \stackrel{\mathrm{qc}(\theta)}{\longrightarrow} \Sigma_{x}+\Sigma_{\rho}
$$

Pela definição de $\hat{\beta}$ e observando que $P_{(k)}^{\prime} T_{(r)}=T_{(k)}^{\prime} S T_{(r)}=\hat{Q}_{(k)}^{\prime} \hat{Q}_{(r)}^{\prime}=0$, obte$\operatorname{mos}^{1}$

$$
\begin{aligned}
\hat{\beta} & =\hat{\Sigma}_{y x} \hat{\Sigma}_{x}^{-1}=P_{r k}\left(\hat{\Lambda}_{(k)}-I\right) P_{k k}^{\prime}\left(P_{k k}^{\prime}\right)^{-1}\left(\hat{\Lambda}_{(k)}-I\right)^{-1} P_{k k}^{-1} \\
& =P_{r k} P_{k k}^{-1}=-\left(T_{r r}^{\prime}\right)^{-1} T_{k r}^{\prime} .
\end{aligned}
$$

Temos que

$$
S^{-1 / 2} S_{Z}=\left(\hat{Q}_{(k)} \hat{\Lambda}_{(k)} \hat{Q}_{(k)}^{\prime}+\hat{Q}_{(r)} \hat{\Lambda}_{(r)} \hat{Q}_{(r)}^{\prime}\right) S^{1 / 2}
$$

e, portanto,

$\operatorname{vec}\left[\left(I, \beta^{\prime}\right) S^{-1} S_{Z}(-\beta, I)^{\prime}\right]=\operatorname{vec}\left[\left(I, \beta^{\prime}\right) S^{-1 / 2}\left(\hat{Q}_{(k)} \hat{\Lambda}_{(k)} \hat{Q}_{(k)}^{\prime}+\hat{Q}_{(r)} \hat{\Lambda}_{(r)} \hat{Q}_{(r)}^{\prime}\right) S^{1 / 2}(-\beta, I)^{\prime}\right]$.

Mas,

$$
\hat{Q}_{(k)} \hat{\Lambda}_{(k)} \hat{Q}_{(k)}^{\prime}=S^{-1 / 2} P_{(k)} \hat{\Lambda}_{(k)} P_{(k)}^{\prime} S^{-1 / 2}
$$

e

$$
\hat{Q}_{(r)} \hat{\Lambda}_{(r)} \hat{Q}_{(r)}^{\prime}=S^{1 / 2} T_{(r)} \hat{\Lambda}_{(r)} T_{(r)}^{\prime} S^{1 / 2}
$$

implicam em

$$
\begin{aligned}
& \left(I, \beta^{\prime}\right) S^{-1 / 2} \hat{Q}_{(k)} \hat{\Lambda}_{(k)} \hat{Q}_{(k)}^{\prime} S^{1 / 2}(-\beta, I)^{\prime} \\
& \quad=\left(I, \beta^{\prime}\right) S^{-1 / 2} S^{-1 / 2} P_{(k)} \hat{\Lambda}_{(k)} P_{(k)}^{\prime} S^{-1 / 2} S^{1 / 2}(-\beta, I)^{\prime} \\
& \quad=\left(I, \beta^{\prime}\right) S^{-1}\left(I, \hat{\beta}^{\prime}\right)^{\prime} P_{k k} \hat{\Lambda}_{(k)} P_{k k}^{\prime}\left(I, \hat{\beta}^{\prime}\right)(-\beta, I)^{\prime} \\
& \quad=\left(I, \beta^{\prime}\right) S^{-1}\left(I, \hat{\beta}^{\prime}\right)^{\prime} P_{k k} \hat{\Lambda}_{(k)} P_{k k}^{\prime}\left(\hat{\beta}^{\prime}-\beta^{\prime}\right) .
\end{aligned}
$$

\footnotetext{
${ }^{1} P_{k k}$ e $T_{r r}$ são invertíveis com probabilidade 1 (ver Gleser, 1981, lemas 2.1 e 2.2).
} 
A segunda igualdade vale por (4.64). Também,

$$
\begin{aligned}
& \left(I, \beta^{\prime}\right) S^{-1 / 2} \hat{Q}_{(r)} \hat{\Lambda}_{(r)} \hat{Q}_{(r)}^{\prime} S^{1 / 2}(-\beta, I)^{\prime} \\
& \quad=\left(I, \beta^{\prime}\right) S^{-1 / 2} S^{1 / 2} T(r) \hat{\Lambda}_{(r)} T_{(r)}^{\prime} S^{1 / 2} S^{1 / 2}(-\beta, I)^{\prime} \\
& \quad=\left(I, \beta^{\prime}\right) T_{(r)} \hat{\Lambda}_{(r)} T_{(r)}^{\prime} S(-\beta, I)^{\prime} \\
& \quad=\left(I, \beta^{\prime}\right)(-\hat{\beta}, I)^{\prime} T_{r r} \hat{\Lambda}_{(r)} T_{r r}^{\prime}(-\hat{\beta}, I) S(-\beta, I)^{\prime} \\
& \quad=-(\hat{\beta}-\beta)^{\prime} T_{r r} \hat{\Lambda}_{(r)} T_{r r}^{\prime}(-\hat{\beta}, I) S(-\beta, I)^{\prime} .
\end{aligned}
$$

Definindo

$$
\begin{aligned}
& F_{k}=\left(I, \beta^{\prime}\right) S^{-1}\left(I, \hat{\beta}^{\prime}\right)^{\prime} P_{k k} \hat{\Lambda}_{(k)} P_{k k}^{\prime} \\
& F_{r}=-T_{r r} \hat{\Lambda}_{(r)} T_{r r}^{\prime}(-\hat{\beta}, I) S(-\beta, I)^{\prime}
\end{aligned}
$$

e usando (4.46), temos que

$$
\begin{aligned}
\operatorname{vec}\left[\left(I, \beta^{\prime}\right) S^{-1} S_{Z}(-\beta, I)^{\prime}\right] & =\operatorname{vec}\left[F_{k}(\hat{\beta}-\beta)^{\prime}+(\hat{\beta}-\beta)^{\prime} F_{r}\right] \\
& =\left(I_{r} \otimes F_{k}+F_{r}^{\prime} \otimes I_{k}\right) \operatorname{vec}(\hat{\beta}-\beta)^{\prime} .
\end{aligned}
$$

Pela consistência de $\hat{\beta}$ e $S$, por (4.61) e por (4.63),

$$
\begin{aligned}
I_{r} \otimes & F_{k}+F_{r}^{\prime} \otimes I_{k} \\
& \stackrel{\mathrm{qc}(\theta)}{\longrightarrow}\left\{I_{r} \otimes\left[(I, \beta)^{\prime} \Sigma_{\epsilon}^{-1}\left(I, \beta^{\prime}\right)^{\prime}\left(\Sigma_{x}+\Sigma_{\rho}\right)\right]\right\}-\left\{\left[(-\beta, I) \Sigma_{\epsilon}(-\beta, I)^{\prime} \Sigma_{\nu}^{-1}\right] \otimes I_{k}\right\} \\
& =I_{r} \otimes\left(\Sigma_{\rho}^{-1} \Sigma_{x}+I_{k}\right)-\left(I_{r} \otimes I_{k}\right) \\
& =I_{r} \otimes\left(\Sigma_{\rho}^{-1} \Sigma_{x}+I_{k}-I_{k}\right)=I_{r} \otimes\left(\Sigma_{\rho}^{-1} \Sigma_{x}\right) .
\end{aligned}
$$

O limite acima é não singular, pois os fatores são não singulares. Então, para $n$ suficientemente grande, com probabilidade $P_{\theta}$ igual a $1, I_{r} \otimes F_{k}+F_{r}^{\prime} \otimes I_{k}$ é invertível.

Vamos analisar a ordem probabilística de

$$
\left(I, \beta^{\prime}\right) S^{-1} S_{Z}(-\beta, I)^{\prime}
$$

Temos, por (4.4), que

$$
S_{Z}=\left(I_{k}, \beta^{\prime}\right)^{\prime} S_{x}\left(I_{k}, \beta^{\prime}\right)+S_{\epsilon x}\left(I_{k}, \beta^{\prime}\right)+\left(I_{k}, \beta^{\prime}\right)^{\prime} S_{x \epsilon}+S_{\epsilon},
$$


e, portanto,

$$
\begin{aligned}
n(I, & \left.\beta^{\prime}\right) S^{-1} S_{Z}(-\beta, I)^{\prime}-n\left(I, \beta^{\prime}\right) \Sigma_{\epsilon}^{-1}\left[\left(I, \beta^{\prime}\right)^{\prime} S_{x \epsilon}+S_{\epsilon}-\Sigma_{\epsilon}\right](-\beta, I)^{\prime} \\
& +n(I, \beta)^{\prime} \Sigma_{\epsilon}^{-1}\left(S-\Sigma_{\epsilon}\right)(-\beta, I)^{\prime} \\
& =n\left(I, \beta^{\prime}\right) S^{-1} S_{Z}(-\beta, I)^{\prime}-n\left(I, \beta^{\prime}\right) \Sigma_{\epsilon}^{-1}\left[S_{Z}-\left(I, \beta^{\prime}\right)^{\prime} S_{x}\left(I, \beta^{\prime}\right)\right. \\
& \left.-S_{\epsilon x}\left(I, \beta^{\prime}\right)-\Sigma_{\epsilon}\right](-\beta, I)^{\prime}+n(I, \beta)^{\prime} \Sigma_{\epsilon}^{-1}\left(S-\Sigma_{\epsilon}\right)(-\beta, I)^{\prime} \\
& =n\left(I, \beta^{\prime}\right) S^{-1} S_{Z}(-\beta, I)^{\prime}-n\left(I, \beta^{\prime}\right) \Sigma_{\epsilon}^{-1}\left(S_{Z}-\Sigma_{\epsilon}\right)(-\beta, I)^{\prime} \\
& +n\left(I, \beta^{\prime}\right) \Sigma_{\epsilon}^{-1}\left(S-\Sigma_{\epsilon}\right)(-\beta, I)^{\prime} \\
& =n(I, \beta)^{\prime}\left[\left(S^{-1}-\Sigma_{\epsilon}^{-1}\right)\left(S_{Z}-\Sigma\right)+S^{-1} \Sigma-\Sigma_{\epsilon}^{-1} \Sigma+\Sigma_{\epsilon}^{-1} S\right](-\beta, I)^{\prime} \\
& =n\left(I, \beta^{\prime}\right)\left[\left(S^{-1}-\Sigma_{\epsilon}^{-1}\right)\left(S_{Z}-\Sigma\right)\right](-\beta, I)^{\prime}+n\left(I, \beta^{\prime}\right)\left[S^{-1} \Sigma_{\epsilon}+\Sigma_{\epsilon}^{-1} S\right](-\beta, I)^{\prime} \\
& =n\left(I, \beta^{\prime}\right)\left[\left(S^{-1}-\Sigma_{\epsilon}^{-1}\right)\left(S_{Z}-\Sigma\right)\right](-\beta, I)^{\prime} \\
& +n\left(I, \beta^{\prime}\right)\left[\left(S^{-1}-\Sigma_{\epsilon}^{-1}\right)\left(S-\Sigma_{\epsilon}\right)-2 I\right](-\beta, I)^{\prime} \\
& =n\left(I, \beta^{\prime}\right)\left[\left(S^{-1}-\Sigma_{\epsilon}^{-1}\right)\left(S_{Z}-\Sigma\right)\right](-\beta, I)^{\prime} \\
& +n\left(I, \beta^{\prime}\right)\left[\left(S^{-1}-\Sigma_{\epsilon}^{-1}\right)\left(S-\Sigma_{\epsilon}\right)\right](-\beta, I)^{\prime} \\
& =O_{p}(1)+O_{p}(1)=O_{p}(1) .
\end{aligned}
$$

A segunda igualdade vale porque $\left(I, \beta^{\prime}\right)(-\beta, I)^{\prime}=0$. A quarta igualdade vale por isso, por (4.7) e por (4.9). A última igualdade é consequência da convergência em distribuição de $n^{1 / 2}\left(\operatorname{vech}\left(S_{Z}-\Sigma\right)\right)$ e de $d^{1 / 2}\left(\operatorname{vech}\left(S-\Sigma_{\epsilon}\right)\right)$, que será provada a seguir, ver (4.77). Assim,

$$
\begin{aligned}
\left(I, \beta^{\prime}\right) S^{-1} S_{Z}(-\beta, I)^{\prime}= & \left(I, \beta^{\prime}\right) \Sigma_{\epsilon}^{-1}\left[\left(I, \beta^{\prime}\right)^{\prime} S_{x \epsilon}+S_{\epsilon}-\Sigma_{\epsilon}\right](-\beta, I)^{\prime} \\
& -\left(I, \beta^{\prime}\right) \Sigma_{\epsilon}^{-1}\left(S-\Sigma_{\epsilon}\right)(-\beta, I)^{\prime}+O_{p}\left(n^{-1}\right) .
\end{aligned}
$$

A primeira e segunda parcelas após a igualdade são da ordem probabilística de $n^{-1 / 2}$, pois

$$
\begin{aligned}
\left(I, \beta^{\prime}\right) \Sigma_{\epsilon}^{-1}\left[\left(I, \beta^{\prime}\right)^{\prime} S_{x \epsilon}+S_{\epsilon}-\Sigma_{\epsilon}\right](-\beta, I)^{\prime} & =\left(I, \beta^{\prime}\right) \Sigma_{\epsilon}^{-1}\left(S_{Z}-\Sigma\right)(-\beta, I)^{\prime} \\
& =O_{p}\left(n^{-1 / 2}\right)
\end{aligned}
$$

e, por (4.78),

$$
\left(I, \beta^{\prime}\right) \Sigma_{\epsilon}^{-1}\left(S_{\epsilon}-\Sigma_{\epsilon}\right)(-\beta, I)^{\prime}=O_{p}\left(n^{-1 / 2}\right) .
$$

Fazendo

$$
\begin{aligned}
S_{x \nu} & =S_{x \epsilon}(-\beta, I)^{\prime}, S_{\xi \nu}=\Sigma_{\rho}\left(I, \beta^{\prime}\right) \Sigma_{\epsilon}^{-1} S_{\epsilon}(-\beta, I)^{\prime} \\
s_{\xi \nu} & =\Sigma_{\rho}\left(I, \beta^{\prime}\right) \Sigma_{\epsilon}^{-1} S(-\beta, I)^{\prime}
\end{aligned}
$$


temos, por (4.70),

$$
\begin{aligned}
\left(I, \beta^{\prime}\right) S^{-1} S_{Z}(-\beta, I)^{\prime} & =\Sigma_{\rho}^{-1}\left(S_{x \nu}+S_{\xi \nu}-s_{\xi \nu}\right)+O_{p}\left(n^{-1}\right) \\
& =O_{p}\left(n^{-1 / 2}\right) .
\end{aligned}
$$

Por (4.67),

$$
\left(I_{r} \otimes F_{k}+F_{r}^{\prime} \otimes I_{k}\right)^{-1} \operatorname{vec}\left[\left(I, \beta^{\prime}\right) S^{-1} S_{Z}(-\beta, I)^{\prime}\right]=\operatorname{vec}(\hat{\beta}-\beta)^{\prime} .
$$

Assim,

$$
\begin{aligned}
\operatorname{vec}( & \hat{\beta}-\beta)^{\prime}=\left[\left(I_{r} \otimes F_{k}+F_{r}^{\prime} \otimes I_{k}\right)^{-1}-\left[I_{r} \otimes\left(\Sigma_{\rho}^{-1} \Sigma_{x}\right)\right]^{-1}\right] \operatorname{vec}\left[\left(I, \beta^{\prime}\right) S^{-1} S_{Z}(-\beta, I)^{\prime}\right] \\
& +\left[I_{r} \otimes\left(\Sigma_{\rho}^{-1} \Sigma_{x}\right)\right]^{-1} \operatorname{vec}\left[\left(I, \beta^{\prime}\right) S^{-1} S_{Z}(-\beta, I)^{\prime}\right] \\
& =o_{p}\left(n^{-1 / 2}\right)+\left[I_{r} \otimes\left(\Sigma_{x}^{-1} \Sigma_{\rho}\right)\right]\left\{\operatorname{vec}\left[\Sigma_{\rho}^{-1}\left(S_{x \nu}+S_{\xi \nu}-s_{\xi \nu}\right)\right]+O_{p}\left(n^{-1}\right)\right\} \\
& =\operatorname{vec}\left[\Sigma_{x}^{-1}\left(S_{x \nu}+S_{\xi \nu}-s_{\xi \nu}\right)\right]+\left[I_{r} \otimes\left(\Sigma_{x}^{-1} \Sigma_{\rho}\right)\right] \times O_{p}\left(n^{-1}\right)+o_{p}\left(n^{-1 / 2}\right) \\
& =\left(I_{r} \otimes \Sigma_{x}^{-1}\right) \operatorname{vec}\left(S_{x \nu}+S_{\xi \nu}-s_{\xi \nu}\right)+o_{p}\left(n^{1 / 2}\right) \times O_{p}\left(n^{-1}\right)+o_{p}\left(n^{-1 / 2}\right) \\
& =\left(I_{r} \otimes \Sigma_{x}^{-1}\right) \operatorname{vec}\left(S_{x \nu}+S_{\xi \nu}-s_{\xi \nu}\right)+o_{p}\left(n^{-1 / 2}\right)
\end{aligned}
$$

Na segunda igualdade usamos (4.68) e (4.71) e na terceira usamos (4.68) e (4.46). O lema a seguir nos ajudará a determinar a normalidade assintótica.

Lema 4.4.1 Suponha que $\left\{\epsilon_{i}, i \in \mathbb{N}\right\} \sim N I I D\left(0, \Sigma_{\epsilon}\right)$ e $\left\{x_{i}, i \in \mathbb{N}\right\} \sim \operatorname{IID}\left(\mu_{x}, \Sigma_{x}\right)$ são conjuntos independentes de vetores aleatórios. Então

$$
n^{1 / 2}\left(\begin{array}{l}
\operatorname{vec} S_{x \epsilon} \\
\operatorname{vech}\left(S_{\epsilon}-\Sigma_{\epsilon}\right)
\end{array}\right) \stackrel{\mathcal{D}}{\longrightarrow} N\left[0, \text { block } \operatorname{diag}\left\{\Sigma_{\epsilon} \otimes \Sigma_{x}, 2 \Delta_{p}\left(\Sigma_{\epsilon} \otimes \Sigma_{\epsilon}\right) \Delta_{p}^{\prime}\right\}\right] .
$$

A demonstração deste lema pode ser encontrada em Amemiya (1982). Temos, por (4.72),

$$
n^{1 / 2} \operatorname{vec}(\hat{\beta}-\beta)^{\prime}=\left(I_{r} \otimes \Sigma_{x}^{-1}\right) \times n^{1 / 2} \operatorname{vec}\left(S_{x \nu}+S_{\xi \nu}-s_{\xi \nu}\right)+o_{p}(1) .
$$

Por (4.4) temos que $S$ é independente de $\left\{\left(x_{i}^{\prime}, \epsilon_{i}^{\prime}\right)^{\prime}, i \in \mathbb{N}\right\}$. Desta forma, $\left\{n^{1 / 2} \operatorname{vec}\left(S_{x \nu}+\right.\right.$ $\left.\left.S_{\xi \nu}\right)\right\}$ é independente de $\left\{n^{1 / 2} \operatorname{vec}\left(s_{\xi \nu}\right)\right\}$. A distribuição limite da diferença $n^{1 / 2}\left[\operatorname{vec}\left(S_{x \nu}\right)+\right.$ $\left.\operatorname{vec}\left(S_{x \nu}\right)\right]-n^{1 / 2} \operatorname{vec}\left(s_{x \nu}\right)$ é dada então pela distribuição da diferença de dois vetores aleatórios independentes, cada um com a distribuição limite de cada parcela. Temos 
que

$$
\begin{aligned}
\operatorname{vec}\left(S_{x \nu}\right) & =\operatorname{vec}\left[S_{x \epsilon}(-\beta, I)^{\prime}\right] \\
& =\left[(-\beta, I) \otimes I_{k}\right] \operatorname{vec}\left(S_{x \epsilon}\right), \\
\operatorname{vec}\left(S_{\xi \nu}\right) & =\left\{(-\beta, I) \otimes\left[\Sigma_{\rho}\left(I, \beta^{\prime}\right)\right]\right\} \operatorname{vec}\left(\Sigma_{\epsilon}^{-1} S_{\epsilon}\right) \\
& =\left\{(-\beta, I) \otimes\left[\Sigma_{\rho}\left(I, \beta^{\prime}\right)\right]\right\}\left\{\left(I_{p} \otimes \Sigma_{\epsilon}^{-1}\right) \operatorname{vec}\left(S_{\epsilon}\right)\right\} \\
& =\left\{(-\beta, I) \otimes\left[\Sigma_{\rho}\left(I, \beta^{\prime}\right) \Sigma_{\epsilon}^{-1}\right]\right\} \operatorname{vec}\left(S_{\epsilon}\right), \\
\operatorname{vec}\left(s_{\xi \nu}\right) & =\left\{(-\beta, I) \otimes\left[\Sigma_{\rho}\left(I, \beta^{\prime}\right) \Sigma_{\epsilon}^{-1}\right]\right\} \operatorname{vec}(S),
\end{aligned}
$$

onde usamos (4.46) para obter a segunda, terceira, a quarta e a sexta igualdades e (4.43) para obter a quinta. Pelo Teorema 3.3.2 de Anderson (1984) e pelo Teorema Central do Limite,

$$
d^{1 / 2} \operatorname{vec}\left(S-\Sigma_{\epsilon}\right) \stackrel{\mathcal{D}(\theta)}{\longrightarrow} N(0, \mathcal{V}),
$$

onde $\mathcal{V}$ é a matriz de covariâncias da distribuição limite de $n^{1 / 2} \operatorname{vec}\left(S_{\epsilon}-\Sigma_{\epsilon}\right)$. A partir do lema 4.4.1 e da $\operatorname{relação~} \operatorname{vec}\left(S_{\epsilon}-\Sigma_{\epsilon}\right)=\Upsilon_{p} \operatorname{vech}\left(S_{\epsilon}-\Sigma_{\epsilon}\right)$ obtemos

$$
\mathcal{V}=2 \Upsilon_{p} \Delta_{p}\left(\Sigma_{\epsilon} \otimes \Sigma_{\epsilon}\right) \Delta_{p}^{\prime} \Upsilon_{p}^{\prime}
$$

Pelo Teorema de Slutsky

$$
n^{1 / 2} \operatorname{vec}\left(S-\Sigma_{\epsilon}\right)=\frac{n^{1 / 2}}{d^{1 / 2}} d^{1 / 2} \operatorname{vec}\left(S-\Sigma_{\epsilon}\right) \stackrel{\mathcal{D}(\theta)}{\longrightarrow} N(0, c \mathcal{V})
$$

Assim,

$$
n^{1 / 2}\left(\begin{array}{l}
\operatorname{vec} S_{x \epsilon} \\
\operatorname{vec}\left(S_{\epsilon}-\Sigma_{\epsilon}\right)
\end{array}\right) \stackrel{\mathcal{D}(\theta)}{\longrightarrow} N\left(0, \text { block } \operatorname{diag}\left\{\Sigma_{\epsilon} \otimes \Sigma_{x}, \Upsilon_{p} \Delta_{p}\left(\Sigma_{\epsilon} \otimes \Sigma_{\epsilon}\right) \Delta_{p}^{\prime} \Upsilon_{p}^{\prime}\right\}\right)
$$

e

$$
n^{1 / 2}\left[\operatorname{vec}\left(S-\Sigma_{\epsilon}\right)\right] \stackrel{\mathcal{D}(\theta)}{\longrightarrow} N\left(0,2 c \Upsilon_{p} \Delta_{p}\left(\Sigma_{\epsilon} \otimes \Sigma_{\epsilon}\right) \Delta_{p}^{\prime} \Upsilon_{p}^{\prime}\right) .
$$

Por (4.74), (4.75), (4.46) e (4.49) vem que

$$
\begin{aligned}
n^{1 / 2} & \operatorname{vec}\left(S_{x \nu}+S_{\xi \nu}\right) \\
= & n^{1 / 2}\left\{\left[(-\beta, I) \otimes I_{k}\right] \operatorname{vec}\left(S_{x \epsilon}\right)+\left[(-\beta, I) \otimes\left[\Sigma_{\rho}\left(I, \beta^{\prime}\right) \Sigma_{\epsilon}^{-1}\right]\right] \operatorname{vec}\left(S_{\epsilon}-\Sigma_{\epsilon}\right)\right\} \\
= & \left.n^{1 / 2}\left\{\left[(-\beta, I) \otimes I_{k}\right] \operatorname{vec}\left(S_{x \epsilon}\right)\right\}+n^{1 / 2}\left\{I_{(k, r)}\left[\Sigma_{\rho}\left(I, \beta^{\prime}\right) \Sigma_{\epsilon}^{-1} \otimes(-\beta, I)\right] I_{(} p, p\right)\right\} \\
& \times \operatorname{vec}\left(S_{\epsilon}-\Sigma_{\epsilon}\right) .
\end{aligned}
$$


Pela definição de $\Delta_{p}$, por (4.55) e por (4.52),

$$
\begin{aligned}
\Upsilon_{p} \Delta_{p}\left(\Sigma_{\epsilon} \otimes \Sigma_{\epsilon}\right) \Delta_{p}^{\prime} \Upsilon_{p}^{\prime} & =\Upsilon_{p} \Delta_{p}\left(\Sigma_{\epsilon} \otimes \Sigma_{\epsilon}\right) \\
& =\frac{1}{2}\left(I_{p^{2}}+I_{(p, p)}\right)\left(\Sigma_{\epsilon} \otimes \Sigma_{\epsilon}\right)
\end{aligned}
$$

Por (4.78) e (4.79), temos que a variância assintótica de $n^{1 / 2} \operatorname{vec}\left(S_{x \nu}+S_{\xi \nu}\right)$ é dada por

$$
\begin{aligned}
{\left[(-\beta, I) \otimes I_{k}\right]\left(\Sigma_{\epsilon} \otimes \Sigma_{x}\right)\left[(-\beta, I)^{\prime} \otimes I_{k}\right]+I_{(k, r)}\left[\Sigma_{\rho}\left(I, \beta^{\prime}\right) \Sigma_{\epsilon}^{-1} \otimes(-\beta, I)\right] I_{(p, p)} } \\
\quad \times\left(I_{p^{2}}+I_{(p, p)}\right)\left(\Sigma_{\epsilon} \otimes \Sigma_{\epsilon}\right) I_{(p, p)}\left[\Sigma_{\epsilon}^{-1}\left(I, \beta^{\prime}\right)^{\prime} \Sigma_{\rho} \otimes(-\beta, I)^{\prime}\right] I_{(r, k)} \\
=\left(\Sigma_{\nu} \otimes \Sigma_{x}\right)+I_{(k, r)}\left[\Sigma_{\rho}\left(I, \beta^{\prime}\right) \Sigma_{\epsilon}^{-1} \otimes(-\beta, I)\right]\left(I_{(p, p)}+I_{p^{2}}\right) \\
\quad \times\left(\Sigma_{\epsilon} \otimes \Sigma_{\epsilon}\right) I_{(p, p)}\left[\Sigma_{\epsilon}^{-1}\left(I, \beta^{\prime}\right)^{\prime} \Sigma_{\rho} \otimes(-\beta, I)^{\prime}\right] I_{(r, k)} \\
=\left(\Sigma_{\nu} \otimes \Sigma_{x}\right)+\left[(-\beta, I) \otimes \Sigma_{\rho}\left(I, \beta^{\prime}\right) \Sigma_{\epsilon}^{-1}\right] \\
\quad \times\left(\Sigma_{\epsilon} \otimes \Sigma_{\epsilon}\right)\left[(-\beta, I)^{\prime} \otimes \Sigma_{\epsilon}^{-1}\left(I, \beta^{\prime}\right)^{\prime} \Sigma_{\rho}\right] \\
\quad+I_{(k, r)}\left[\Sigma_{\rho}\left(I, \beta^{\prime}\right) \Sigma_{\epsilon}^{-1} \otimes(-\beta, I)\right]\left(\Sigma_{\epsilon} \otimes \Sigma_{\epsilon}\right) \\
\quad \times\left[(-\beta, I)^{\prime} \otimes \Sigma_{\epsilon}^{-1}\left(I, \beta^{\prime}\right)^{\prime} \Sigma_{\rho}\right] \\
\quad=\left(\Sigma_{\nu} \otimes \Sigma_{x}\right)+\left(\Sigma_{\nu} \otimes \Sigma_{\rho}\right) .
\end{aligned}
$$

A primeira igualdade vale por (4.48), a segunda vale por (4.49) e a última vale porque, por (4.49) e (4.43), o termo em colchetes imediatamente antes dessa igualdade é igual a zero. Analogamente provamos que a variância assintótica de $n^{1 / 2} \operatorname{vec}\left(s_{\xi \nu}\right)$ é dada por $c\left(\Sigma_{\nu} \otimes \Sigma_{\rho}\right)$. Assim, por (4.73), a distribuição assintótica de $n^{1 / 2} \operatorname{vec}(\hat{\beta}-\beta)^{\prime}$ é normal com média zero e matriz de covariância

$$
\begin{aligned}
\left(I_{r}\right. & \left.\otimes \Sigma_{x}^{-1}\right)\left[\Sigma_{\nu} \otimes \Sigma_{x}+(1+c)\left(\Sigma_{\nu} \otimes \Sigma_{\rho}\right)\right]\left(I_{r} \otimes \Sigma_{x}^{-1}\right) \\
& =\left(I_{r} \otimes \Sigma_{x}^{-1}\right)\left(\Sigma_{\nu} \otimes \Sigma_{x}\right)\left(I_{r} \otimes \Sigma_{x}^{-1}\right)+(1+c)\left(I_{r} \otimes \Sigma_{x}^{-1}\right)\left(\Sigma_{\nu} \otimes \Sigma_{\rho}\right)\left(I_{r} \otimes \Sigma_{x}^{-1}\right) \\
& =\left(\Sigma_{\nu} \otimes \Sigma_{x}^{-1}\right)+(1+c)\left(\Sigma_{\nu} \otimes \Sigma_{x}^{-1} \Sigma_{\rho} \Sigma_{x}^{-1}\right) .
\end{aligned}
$$

Como $\operatorname{vec}(\hat{\beta}-\beta)=I_{(k, r)} \operatorname{vec}(\hat{\beta}-\beta)^{\prime}$, temos, por (4.49), a validade do Teorema a seguir.

Teorema 4.4.1 Suponha válidas (4.1), (4.2), (1), (2) e (3). Seja $\hat{\beta}$ o estimador de máxima verossimilhança para $\beta$, definido no Teorema 4.1.2. Então, para todo $\theta \in \Theta^{*}$,

$$
n^{1 / 2} \operatorname{vec}(\hat{\beta}-\beta) \stackrel{\mathcal{D}(\theta)}{\longrightarrow} N\left[0, \Sigma_{x}^{-1} \otimes \Sigma_{\nu}+(1+c)\left(\Sigma_{x}^{-1} \Sigma_{\rho} \Sigma_{x}^{-1} \otimes \Sigma_{\nu}\right)\right] .
$$




\subsection{Testes de Distância para Algumas Hipóteses Restritas}

Nesta seção exibiremos estudos de poder, utilizando dados simulados, relacionados a testes para algumas hipóteses restritas em torno do parâmetro vec $(\beta)$. Usaremos os resultados da seção anterior e a teoria geral apresentada no capítulo 2 .

Considere então um MRME satisfazendo as condições necessárias para a validade do Teorema 4.4.1, e as hipóteses

$$
H: h(\operatorname{vec}(\beta))=0, \quad K: h(\operatorname{vec}(\beta)) \geq 0 .
$$

A estatística para o teste de distância de $H$ contra $K$ é dada por

$$
\mathbf{D}=\left\|\pi_{\hat{\Sigma}_{\beta}}\left(n^{1 / 2} h(\operatorname{vec}(\hat{\beta})), \mathcal{O}_{l}\right),\right\|_{\hat{\Sigma}_{\beta}}^{2}
$$

onde $l$ é a dimensão do vetor $h(\operatorname{vec}(\hat{\beta}))$ e $\hat{\Sigma}_{\beta}$ é o estimador consistente da matriz de covariâncias da distribuição limite no teorema 4.4.1, ou seja, o estimador obtido substituindo os elementos na expressão desta matriz pelos estimadores de máxima verossimilhança correspondentes. No apêndice A mostramos qual o método computacional que utilizamos no cálculo de $\mathbf{D}$.

Para efeito deste estudo, considere o MRME com $r=1$ e $k=2$, ou seja, com resposta unidimensional e dois regressores. Escrevendo $\beta=\left(\beta_{1}, \beta_{2}\right)$, estudaremos o comportamento, em amostras finitas, do teste de distância para

$$
H: \beta_{1}=\beta_{2}=1 \quad \text { contra } \quad K: \beta_{1} \geq \beta_{2} \geq 1 .
$$

Utilizando os estimadores consistentes dos parâmetros do MRME definidos no teorema 4.1.2, temos que a estatística de teste é dada em $(4.80), \operatorname{com} h(\operatorname{vec}(\hat{\beta}))=$ $\left(\hat{\beta}_{1}-\hat{\beta}_{2}, \hat{\beta}_{2}-1\right)^{\prime}$. O procedimento de teste utilizado é o descrito no capítulo 2 , após a demonstração do teorema 2.2.4. É feita uma comparação com o teste de Wald usual e com um teste exato elaborado por Wolak (1987), o qual passaremos a descrever agora. Este teste assume o modelo de regressão linear clássico, onde supõe-se que os regressores são observados sem erro, e por isso será denominado teste "naive". Utilizamos a notação do trabalho original.

O modelo de regressão linear clássico é definido por

$$
Y=X \beta+\epsilon
$$


onde o vetor de erros de observação $\epsilon: n \times 1$ tem distribuição $N\left(0, \sigma^{2} I\right), \sigma^{2}>0$ (desconhecido), $X: n \times q$ é uma matriz de constantes com $\operatorname{posto}(X)=q<n$, $\beta: q \times 1$ é um vetor de parâmetros a ser estimado. É bem conhecido que o estimador de mínimos quadrados para $\beta$ é

$$
\hat{\beta}=\left(X^{\prime} X\right)^{-1} X^{\prime} Y,
$$

e que um estimador não viciado para a variância dos erros, $\sigma^{2}$, é dado por

$$
S^{2}=\frac{1}{n-q}\|Y-X \hat{\beta}\|^{2} .
$$

Temos que $(n-q) S^{2} / \sigma^{2}$ tem distribuição qui-quadrado com $n-q$ graus de liberdade e $\hat{\beta}$ e $S^{2}$ são independentes. Considere o problema de testar

$$
H: C \beta=b \quad \text { contra } \quad K: C \beta \geq b,
$$

onde $C: m \times q$ tem posto $m$. Suponha por um momento que $\sigma^{2}$ é conhecido. Neste caso, a estatística do teste da razão de verossimilhança é dada por

$$
R=\frac{1}{\sigma^{2}}\left(\min \left\{\|Y-X \beta\|^{2} ; C \beta=b\right\}-\min \left\{\|Y-X \beta\|^{2} ; C \beta \geq b\right\}\right),
$$

e rejeitamos $H$ para grandes valores de $R$. A distribuição nula de $R$ é dada por

$$
P(R \geq c)=\sum_{j=0}^{m} P\left(\chi_{j}^{2} \geq c\right) w\left(m, j, C\left(X^{\prime} X\right)^{-1} C^{\prime}\right) .
$$

Os pesos na mistura acima foram definidos no capítulo 2. Uma demonstração desse resultado pode ser encontrada em Shapiro (1988). O teste sugerido por Wolak (1987) é baseado na estatística

$$
R V=\frac{\sigma^{2} R}{S^{2}}
$$

Para encontrar a distribuição nula de $R V$, observe primeiramente que, para todo $\beta \in \mathbb{R}^{q}$

$$
\|Y-X \beta\|^{2}=\|Y-X \hat{\beta}\|^{2}+\|X(\hat{\beta}-\beta)\|^{2},
$$

pois $Y-X \hat{\beta}$ é ortogonal ao espaço gerado pelas colunas de $X$. Assim, podemos escrever

$$
\sigma^{2} R=\min \left\{\|X(\hat{\beta}-\beta)\|^{2} ; C \beta=b\right\}-\min \left\{\|X(\hat{\beta}-\beta)\|^{2} ; C \beta \geq b\right\},
$$


e, conseqüentemente, $\sigma^{2} R$ é independente de $S^{2}$.

Seja $\Omega=C\left(X^{\prime} X\right)^{-1} C^{\prime}$ e $W \sim N(0, \Omega)$ uma variável aleatória independente de $S^{2}$. A distribuição de $R$ é igual à distribuição de $\left\|\pi_{\Omega}\left(W, \mathcal{O}_{m}\right)\right\|_{\Omega}^{2}$, e a distribuição nula de $\sigma^{2} R / S^{2}$ é a mesma de

$$
\frac{\sigma^{2}}{S^{2}}\left\|\pi_{\Omega}\left(W, \mathcal{O}_{m}\right)\right\|_{\Omega}^{2}=\left\|\pi_{S^{2} \Omega / \sigma^{2}}\left(W, \mathcal{O}_{m}\right)\right\|_{S^{2} \Omega / \sigma^{2}}^{2} .
$$

A abordagem a seguir é análoga à discussão que sucede o corolário 2.2.1. Tome então $M \subset\{1, \ldots, m\}$ (com $t$ elementos), $\mathcal{X}_{M}$ e particione $W=\left(W_{1}^{\prime}, W_{2}^{\prime}\right)^{\prime}, W_{2}: t \times 1$, $\Omega=\left(\Omega_{i j}\right), \Omega_{22}: t \times t$, como naquele instante. Temos que

$$
\begin{aligned}
& P\left(\frac{\sigma^{2}}{S^{2}}\left\|\pi_{\Omega}\left(W, \mathcal{O}_{m}\right)\right\|_{\Omega}^{2} \geq c\right)= \\
& \quad \sum_{M} P\left(\frac{\sigma^{2}}{S^{2}}\left\|\pi_{\Omega}\left(W, \mathcal{O}_{m}\right)\right\|_{\Omega}^{2} \geq c \mid \pi_{\Omega}\left(W, \mathcal{O}_{m}\right) \in \chi_{M}\right) .
\end{aligned}
$$

Mas

$$
\begin{aligned}
& P\left(\frac{\sigma^{2}}{S^{2}}\left\|\pi_{\Omega}\left(W, \mathcal{O}_{m}\right)\right\|_{\Omega}^{2} \geq c, \pi_{\Omega}\left(W, \mathcal{O}_{m}\right) \in \mathcal{X}_{M}\right) \\
& \quad=P\left(\frac{\sigma^{2}}{S^{2}}\left\|\pi_{\Omega}\left(W, \mathcal{O}_{m}\right)\right\|_{\Omega}^{2} \geq c, W_{2}-\Omega_{21} \Omega_{11}^{-1} W_{1}>0, \Omega_{11}^{-1} W_{1} \leq 0\right) \\
& \quad=P\left(\frac{\sigma^{2}}{S^{2}}\left\|W_{2}-\Omega_{21} \Omega_{11}^{-1} W_{1}\right\|_{\Omega_{22.1}}^{2} \geq c, W_{2}-\Omega_{21} \Omega_{11}^{-1} W_{1}>0, \Omega_{11}^{-1} W_{1} \leq 0\right) \\
& \quad=E\left[P\left(\frac{\sigma^{2}}{S^{2}}\left\|W_{2}-\Omega_{21} \Omega_{11}^{-1} W_{1}\right\|_{\Omega_{22.1}}^{2} \geq c, W_{2}-\Omega_{21} \Omega_{11}^{-1} W_{1}>0, \Omega_{11}^{-1} W_{1} \leq 0 \mid S\right)\right],
\end{aligned}
$$

onde $E$ denota esperanças. Usando o "princípio da substituição" (Ash, 1972, pg. 266), a independência entre $W_{1}$ e $W_{2}-\Omega_{21} \Omega_{11}^{-1} W_{1}$ e o corolário 2.1.1, vem que

$$
\begin{aligned}
& P\left(\frac{\sigma^{2}}{S^{2}}\left\|W_{2}-\Omega_{21} \Omega_{11}^{-1} W_{1}\right\|_{\Omega_{22.1}}^{2} \geq c, W_{2}-\Omega_{21} \Omega_{11}^{-1} W_{1}>0, \Omega_{11}^{-1} W_{1} \leq 0 \mid S=s\right) \\
& \quad=P\left(\frac{\sigma^{2}}{s^{2}}\left\|W_{2}-\Omega_{21} \Omega_{11}^{-1} W_{1}\right\|_{\Omega_{22.1}}^{2} \geq c, W_{2}-\Omega_{21} \Omega_{11}^{-1} W_{1}>0, \Omega_{11}^{-1} W_{1} \leq 0 \mid S=s\right) \\
& \quad=P\left(\frac{\sigma^{2}}{s^{2}}\left\|W_{2}-\Omega_{21} \Omega_{11}^{-1} W_{1}\right\|_{\Omega_{22.1}}^{2} \geq c, W_{2}-\Omega_{21} \Omega_{11}^{-1} W_{1}>0, \Omega_{11}^{-1} W_{1} \leq 0\right) \\
& \quad=P\left(\frac{\sigma^{2}}{s^{2}}\left\|W_{2}-\Omega_{21} \Omega_{11}^{-1} W_{1}\right\|_{\Omega_{22.1}}^{2} \geq c\right) P\left(W_{2}-\Omega_{21} \Omega_{11}^{-1} W_{1}>0\right) P\left(\Omega_{11}^{-1} W_{1} \leq 0\right) .
\end{aligned}
$$

Portanto, (4.83) é igual a

$$
P\left(\left\|W_{2}-\Omega_{21} \Omega_{11}^{-1} W_{1}\right\|_{\Omega_{22.1}}^{2} \geq c \frac{S^{2}}{\sigma^{2}}\right) P\left(W_{2}-\Omega_{21} \Omega_{11}^{-1} W_{1}>0\right) P\left(\Omega_{11}^{-1} W_{1} \leq 0\right) .
$$

Como $\left\|W_{2}-\Omega_{21} \Omega_{11}^{-1} W_{1}\right\|_{\Omega_{22.1}}^{2} \sim \chi_{t}^{2},(n-q) S^{2} / \sigma^{2} \sim \chi_{n-q}^{2}$ e $W$ e $S^{2}$ são independentes, temos que a probabilidade condicional em (4.82) é igual a

$$
P\left(\frac{\frac{t}{t}\left\|W_{2}-\Omega_{21} \Omega_{11}^{-1} W_{1}\right\|_{\Omega_{22.1}}^{2}}{\frac{(n-q) S^{2}}{(n-q) \sigma^{2}}} \geq c\right)=P\left(F_{t, n-q} \geq c / t\right), \quad t=1, \ldots, m,
$$


onde $F_{t, n-q}$ representa uma variável aleatória com distribuição $F$ de Snedecor com $t$ graus de liberdade para o numerador e $n-q$ graus de liberdade para o denominador. Assim, a distribuição nula de $R V$ é dada por

$$
P(R V \geq c)=\sum_{t=1}^{m} P\left(F_{t, n-q} \geq c / t\right) w(m, t, \Omega), \quad \text { se } c>0
$$

e

$$
P(R V=0)=w(m, 0, \Omega) .
$$

Os resultados do estudo de simulação são apresentados a seguir. Cada elemento na tabela 4.1 corresponde à taxa de rejeição de $H$ (definida em 4.81) para os testes de distância, Wald e "naive" em 2000 amostras do MRME simuladas de acordo com os parâmetros especificados. Façamos

$$
\Sigma_{\epsilon}=\operatorname{block} \operatorname{diag}\left\{\Sigma_{\delta}, \Sigma_{e}\right\}
$$

onde $\Sigma_{\delta}$ é a matriz de covariâncias dos erros de observação nas variáveis regressoras e $\Sigma_{e}$ a matriz de covariância dos erros de observação no vetor de resposta. Em todas as amostras geradas tomamos $\alpha=1, \mu_{x}=(1,1)^{\prime}, \beta_{2}=1$ e $\Sigma_{e}=0.5$. Para o estimador $S$ definido em (4.10) tomamos sempre $d=30$. Novamente temos uma performance superior do teste de distância; a inadequação do teste "naive" é clara. Na tabela 4.2, tentamos obter uma idéia do comportamento das distribuições nulas das estatísticas de teste como função da razão de confiabilidade, definida por $^{2}$

$$
\kappa_{x}=\left(\Sigma_{x}+\Sigma_{\delta}\right)^{-1} \Sigma_{x}
$$

Como, nos experimentos simulados, tomamos sempre $\Sigma_{x}$ e $\Sigma_{\delta}$ diagonais, com elementos iguais na diagonal principal, vem que $\kappa_{x}$ é da forma $\operatorname{diag}\{\kappa, \kappa\}$. A aproximação às distribuições limite parece piorar, a medida que as variâncias dos erros de observação nos regressores aumentam. Isto ocorre notadamente no caso do teste de distância.

\footnotetext{
${ }^{2}$ No caso em que $\Sigma_{\delta}$ e $\Sigma_{x}$ são matrizes diagonais, os elementos da diagonal desta matriz representam, segundo Fuller (1987, pg. 3), a "atenuação" sofrida pelo coeficiente de regressão devido ao erro de observação.
} 
Tabela 4.1: Taxas de Rejeição para os Testes de Distância, Wald Irrestrito e Naive $\left(n=60, H: \beta_{1}=\right.$ $\beta_{2}=1, K: \beta_{1} \geq \beta_{2} \geq 1$ )

\begin{tabular}{|c|c|c|c|c|c|c|}
\hline \multirow[t]{2}{*}{$\Sigma_{\delta}$} & \multirow[t]{2}{*}{$\Sigma_{x}$} & \multirow[t]{2}{*}{ Nível } & \multirow[t]{2}{*}{$\beta_{1}$} & \multicolumn{3}{|c|}{ Teste } \\
\hline & & & & Distância & Wald & "Naive" \\
\hline \multirow[t]{28}{*}{$\operatorname{diag}\{0.1,0.1\}$} & $\operatorname{diag}\{0.2,0.2\}$ & $5 \%$ & 1.00 & 0.041 & 0.081 & 0.000 \\
\hline & $(\kappa=0.67)$ & & 1.05 & 0.050 & 0.072 & 0.000 \\
\hline & & & 1.10 & 0.055 & 0.063 & 0.000 \\
\hline & & & 1.15 & 0.083 & 0.075 & 0.002 \\
\hline & & & 1.20 & 0.096 & 0.069 & 0.002 \\
\hline & & & 1.25 & 0.128 & 0.092 & 0.002 \\
\hline & & & 1.30 & 0.217 & 0.164 & 0.009 \\
\hline & & & 1.35 & 0.187 & 0.123 & 0.013 \\
\hline & & & 1.40 & 0.224 & 0.156 & 0.013 \\
\hline & & $10 \%$ & 1.00 & 0.060 & 0.134 & 0.001 \\
\hline & & & 1.05 & 0.085 & 0.128 & 0.001 \\
\hline & & & 1.10 & 0.095 & 0.118 & 0.001 \\
\hline & & & 1.15 & 0.134 & 0.134 & 0.003 \\
\hline & & & 1.20 & 0.136 & 0.130 & 0.004 \\
\hline & & & 1.25 & 0.191 & 0.155 & 0.009 \\
\hline & & & 1.30 & 0.217 & 0.164 & 0.018 \\
\hline & & & 1.35 & 0.253 & 0.198 & 0.026 \\
\hline & & & 1.40 & 0.308 & 0.239 & 0.037 \\
\hline & $\operatorname{diag}\{0.3,0.3\}$ & $5 \%$ & 1.00 & 0.038 & 0.074 & 0.001 \\
\hline & $(\kappa=0.75)$ & & 1.05 & 0.063 & 0.065 & 0.002 \\
\hline & & & 1.10 & 0.083 & 0.077 & 0.003 \\
\hline & & & 1.15 & 0.110 & 0.075 & 0.004 \\
\hline & & & 1.20 & 0.155 & 0.097 & 0.009 \\
\hline & & & 1.25 & 0.211 & 0.135 & 0.012 \\
\hline & & & 1.30 & 0.253 & 0.171 & 0.040 \\
\hline & & & 1.35 & 0.333 & 0.213 & 0.036 \\
\hline & & & 1.40 & 0.393 & 0.264 & 0.067 \\
\hline & & $10 \%$ & 1.00 & 0.064 & 0.124 & 0.004 \\
\hline
\end{tabular}




\begin{tabular}{|c|c|c|c|c|c|c|}
\hline \multicolumn{7}{|c|}{ continuação da página anterior } \\
\hline \multirow[t]{2}{*}{$\Sigma_{\delta}$} & \multirow[t]{2}{*}{$\Sigma_{x}$} & \multirow[t]{2}{*}{ Nível } & \multirow[t]{2}{*}{$\beta_{1}$} & \multicolumn{3}{|c|}{ Teste } \\
\hline & & & & Distância & Wald & "Naive" \\
\hline & & & 1.05 & 0.107 & 0.124 & 0.005 \\
\hline & & & 1.10 & 0.138 & 0.136 & 0.008 \\
\hline & & & 1.15 & 0.164 & 0.138 & 0.008 \\
\hline & & & 1.20 & 0.215 & 0.175 & 0.022 \\
\hline & & & 1.25 & 0.286 & 0.218 & 0.034 \\
\hline & & & 1.30 & 0.330 & 0.258 & 0.065 \\
\hline & & & 1.35 & 0.425 & 0.324 & 0.070 \\
\hline & & & 1.40 & 0.496 & 0.372 & 0.123 \\
\hline & $\operatorname{diag}\{0.4,0.4\}$ & $5 \%$ & 1.00 & 0.039 & 0.065 & 0.001 \\
\hline & $(\kappa=0.80)$ & & 1.05 & 0.063 & 0.065 & 0.003 \\
\hline & & & 1.10 & 0.105 & 0.073 & 0.003 \\
\hline & & & 1.15 & 0.136 & 0.093 & 0.009 \\
\hline & & & 1.20 & 0.192 & 0.121 & 0.015 \\
\hline & & & 1.25 & 0.277 & 0.166 & 0.038 \\
\hline & & & 1.30 & 0.356 & 0.215 & 0.065 \\
\hline & & & 1.35 & 0.430 & 0.286 & 0.087 \\
\hline & & & 1.40 & 0.393 & 0.264 & 0.130 \\
\hline & & $10 \%$ & 1.00 & 0.071 & 0.117 & 0.002 \\
\hline & & & 1.05 & 0.109 & 0.127 & 0.006 \\
\hline & & & 1.10 & 0.149 & 0.130 & 0.009 \\
\hline & & & 1.15 & 0.185 & 0.150 & 0.021 \\
\hline & & & 1.20 & 0.271 & 0.196 & 0.043 \\
\hline & & & 1.25 & 0.365 & 0.266 & 0.070 \\
\hline & & & 1.30 & 0.445 & 0.327 & 0.108 \\
\hline & & & 1.35 & 0.526 & 0.401 & 0.155 \\
\hline & & & 1.40 & 0.603 & 0.466 & 0.215 \\
\hline \multirow[t]{5}{*}{$\operatorname{diag}\{0.2,0.2\}$} & $\operatorname{diag}\{0.3,0.3\}$ & $5 \%$ & 1.00 & 0.024 & 0.081 & 0.000 \\
\hline & $(\kappa=0.60)$ & & 1.05 & 0.035 & 0.069 & 0.000 \\
\hline & & & 1.10 & 0.038 & 0.065 & 0.000 \\
\hline & & & 1.15 & 0.066 & 0.068 & 0.001 \\
\hline & & & 1.20 & 0.084 & 0.082 & 0.001 \\
\hline
\end{tabular}




\begin{tabular}{|c|c|c|c|c|c|c|}
\hline \multicolumn{7}{|c|}{ continuação da página anterior } \\
\hline \multirow[t]{2}{*}{$\Sigma_{\delta}$} & \multirow[t]{2}{*}{$\Sigma_{x}$} & \multirow[t]{2}{*}{ Nível } & \multirow[t]{2}{*}{$\beta_{1}$} & \multicolumn{3}{|c|}{ Teste } \\
\hline & & & & Distância & Wald & "Naive" \\
\hline & & & 1.25 & 0.110 & 0.083 & 0.001 \\
\hline & & & 1.30 & 0.123 & 0.084 & 0.002 \\
\hline & & & 1.35 & 0.178 & 0.120 & 0.004 \\
\hline & & & 1.40 & 0.196 & 0.129 & 0.004 \\
\hline & & $10 \%$ & 1.00 & 0.049 & 0.140 & 0.000 \\
\hline & & & 1.05 & 0.067 & 0.128 & 0.001 \\
\hline & & & 1.10 & 0.071 & 0.106 & 0.001 \\
\hline & & & 1.15 & 0.110 & 0.125 & 0.001 \\
\hline & & & 1.20 & 0.127 & 0.145 & 0.001 \\
\hline & & & 1.25 & 0.164 & 0.157 & 0.002 \\
\hline & & & 1.30 & 0.185 & 0.155 & 0.006 \\
\hline & & & 1.35 & 0.252 & 0.196 & 0.007 \\
\hline & & & 1.40 & 0.270 & 0.211 & 0.009 \\
\hline & $\operatorname{diag}\{0.4,0.4\}$ & $5 \%$ & 1.00 & 0.036 & 0.076 & 0.000 \\
\hline & $(\kappa=0.67)$ & & 1.05 & 0.048 & 0.073 & 0.000 \\
\hline & & & 1.10 & 0.054 & 0.069 & 0.001 \\
\hline & & & 1.15 & 0.095 & 0.082 & 0.001 \\
\hline & & & 1.20 & 0.112 & 0.086 & 0.001 \\
\hline & & & 1.25 & 0.166 & 0.105 & 0.003 \\
\hline & & & 1.30 & 0.204 & 0.130 & 0.006 \\
\hline & & & 1.35 & 0.255 & 0.167 & 0.007 \\
\hline & & & 1.40 & 0.326 & 0.219 & 0.016 \\
\hline & & $10 \%$ & 1.00 & 0.061 & 0.139 & 0.000 \\
\hline & & & 1.05 & 0.084 & 0.123 & 0.001 \\
\hline & & & 1.10 & 0.094 & 0.118 & 0.002 \\
\hline & & & 1.15 & 0.147 & 0.145 & 0.002 \\
\hline & & & 1.20 & 0.166 & 0.152 & 0.002 \\
\hline & & & 1.25 & 0.234 & 0.189 & 0.007 \\
\hline & & & 1.30 & 0.275 & 0.215 & 0.016 \\
\hline & & & 1.35 & 0.336 & 0.260 & 0.020 \\
\hline & & & 1.40 & 0.418 & 0.325 & 0.038 \\
\hline
\end{tabular}




\begin{tabular}{lccccc}
\hline continuação da página anterior & & & & \\
\hline$\Sigma_{\delta}$ & Nível & $\beta_{1}$ & \multicolumn{3}{c}{ Teste } \\
& & & Distância & Wald & "Naive" \\
\hline \multirow{2}{*}{$(\kappa=0.71)$} & & 1.05 & 0.052 & 0.062 & 0.001 \\
& & 1.10 & 0.072 & 0.067 & 0.001 \\
& & 1.15 & 0.119 & 0.083 & 0.002 \\
& & 1.20 & 0.177 & 0.118 & 0.003 \\
& & 1.25 & 0.221 & 0.146 & 0.010 \\
& & 1.30 & 0.301 & 0.187 & 0.013 \\
& & 1.35 & 0.368 & 0.243 & 0.019 \\
& & 1.40 & 0.450 & 0.309 & 0.029 \\
\cline { 2 - 6 } & $10 \%$ & 1.00 & 0.070 & 0.125 & 0.001 \\
& & 1.05 & 0.087 & 0.119 & 0.001 \\
& 1.10 & 0.121 & 0.121 & 0.001 \\
& & 1.15 & 0.174 & 0.143 & 0.003 \\
& 1.20 & 0.247 & 0.193 & 0.007 \\
& & 1.25 & 0.296 & 0.232 & 0.019 \\
& 1.30 & 0.388 & 0.286 & 0.026 \\
& 1.35 & 0.462 & 0.359 & 0.043 \\
& 1.40 & 0.546 & 0.426 & 0.063 \\
\hline & & & &
\end{tabular}


Tabela 4.2: Taxas de Rejeição sob a Hipótese Nula para Alguns Valores de $\kappa$ ( $H$ : $\left.\beta_{1}=\beta_{2}=1, \Sigma_{x}=\operatorname{diag}\{0.2,0.2\}\right)$

\begin{tabular}{llllcc}
\hline$n$ & Nível nominal & $\Sigma_{\delta i i}$ & $\kappa$ & \multicolumn{2}{c}{ Teste } \\
& & & & Distância & Wald \\
\hline 100 & $5 \%$ & 0.12 & 0.6250 & 0.0325 & 0.0695 \\
& & 0.14 & 0.5882 & 0.0280 & 0.0725 \\
& & 0.16 & 0.5555 & 0.0210 & 0.0740 \\
& & 0.18 & 0.5263 & 0.0165 & 0.0790 \\
& & 0.20 & 0.5000 & 0.0130 & 0.0840 \\
\hline $10 \%$ & 0.12 & 0.6250 & 0.0585 & 0.1215 \\
& & 0.14 & 0.5882 & 0.0475 & 0.1230 \\
& & 0.16 & 0.5555 & 0.0420 & 0.1245 \\
& & 0.18 & 0.5263 & 0.0355 & 0.1290 \\
& 0.20 & 0.5000 & 0.0320 & 0.1305 \\
\hline 500 & $5 \%$ & 0.12 & 0.6250 & 0.0370 & 0.0990 \\
& & 0.14 & 0.5882 & 0.0300 & 0.1035 \\
& 0.16 & 0.5555 & 0.0250 & 0.1030 \\
& 0.18 & 0.5263 & 0.0205 & 0.1060 \\
& 0.20 & 0.5000 & 0.0170 & 0.1080 \\
\hline $10 \%$ & 0.12 & 0.6250 & 0.0645 & 0.1515 \\
& & 0.14 & 0.5882 & 0.0590 & 0.1570 \\
& 0.16 & 0.5555 & 0.0505 & 0.1550 \\
& 0.18 & 0.5263 & 0.0440 & 0.1555 \\
& 0.20 & 0.5000 & 0.0380 & 0.1590 \\
\hline & & & & & \\
& & & &
\end{tabular}




\section{Capítulo 5}

\section{Testes de Distância sob Condições Não-regulares: Aplicações ao Modelo de Calibração Comparativa}

Neste capítulo apresentamos o modelo de regressão linear múltipla, multivariado e com matriz de covariâncias dos erros de observação conhecida, a menos de uma constante (MRMC). Obtemos o estimador de máxima verossimilhança para o vetor de parâmetros do modelo, e mostramos a sua consistência e normalidade assintótica. Em um caso particular do MRMC, o modelo de calibração comparativa, verificamos a inadequação dos testes de Wald e Distância para algumas hipóteses relevantes, pois algumas funções dos estimadores de máxima verossimilhança podem apresentar variância assintótica nula. Uma modificação no teste de Distância é sugerida e, através de simulações por computador, mostra-se eficiente para contornar o problema.

\subsection{Definição do Modelo}

O modelo de regressão linear multivariado é adequado para a análise do seguinte procedimento experimental, denominado calibração comparativa de instrumentos ${ }^{1}$ : considere uma amostra aleatória com $n$ unidades experimentais obtida de uma determinada população. Suponha que uma característica numérica (unidimensional)

\footnotetext{
${ }^{1}$ Veja em Williams (1969) as diferenças entre calibração comparativa e calibração absoluta.
} 
da população é observada em cada unidade a partir de mensurações realizadas por $p$ instrumentos. Em uma determinada unidade, cada instrumento mede a característica uma única vez. Existe um instrumento que faz as mensurações sem vício (a exata definição de vício será dada a seguir) e, devido a isso, é denominado intrumento padrão. Os outros instrumentos podem apresentar vício e, portanto, suas mensurações devem ser corrigidas (calibradas), de modo a eliminar este problema.

Seja $x_{i}$ o verdadeiro valor da característica na unidade $i . X_{i}$ denotará o valor da mensuração feita pelo instrumento padrão, $Y_{i}=\left(Y_{i 1}, \ldots, Y_{i r}\right)^{\prime}$ o vetor com as mensurações feitas pelos instrumentos restantes e $Z_{i}=\left(X_{i}, Y_{i}^{\prime}\right)^{\prime}$ o vetor de observações, $i=1, \ldots, n$. Façamos $p=r+1$. Adotaremos (4.1) e (4.2) para definir a relação funcional entre $Z_{i}$ e $x_{i}$. Escrevendo $\alpha=\left(\alpha_{1}, \ldots, \alpha_{r}\right)^{\prime}$ e $\beta=\left(\beta_{1}, \ldots, \beta_{r}\right)^{\prime}$, as coordenadas $\alpha_{j}$ e $\beta_{j}$ serão denominadas vício aditivo $e$ multiplicativo, respectivamente, associados ao instrumento $j, j=1, \ldots, r$. Assumiremos (4.3) com uma modificação: suporemos que a variância dos erros de observação $\epsilon_{i}=\left(\delta_{i}, e_{i 1}, \ldots, e_{i r}\right)^{\prime}$ é $\sigma^{2} \Omega$, com $\sigma^{2}>0$ desconhecido e $\Omega>0$ conhecida. O modelo definido por esta estrutura é denominado modelo de calibração comparativa. Para $\beta_{j} \neq 0$, definimos uma mensuração calibrada feita na unidade $i$ pelo instrumento $j$ por

$$
\left(Y_{i j}-\alpha_{j}\right) / \beta_{j}=x_{i}+e_{i j} / \beta_{j}
$$

$i=1, \ldots, n, j=1, \ldots, r$. Na prática, as mensurações calibradas são obtidas substituindo os parâmetros por estimadores no lado esquerdo de (5.1). Instrumentos perfeitamente calibrados como em (5.1) fornecem medidas sem vício. Assim, um critério de comparação entre os instrumentos é a comparação entre os recíprocos das variâncias dos erros (das medidas calibradas) $\delta_{i}, e_{i 1} / \beta_{1}, \ldots, e_{i r} / \beta_{r}$, as precisões, dadas por

$$
1 /\left(\sigma^{2} \Omega_{11}\right), \beta_{1}^{2} /\left(\sigma^{2} \Omega_{22}\right), \ldots, \beta_{r}^{2} /\left(\sigma^{2} \Omega_{p p}\right)
$$

respectivamente.

Alguns exemplos de hipóteses relevantes que interessa-nos testar: para dois instrumentos quaisquer, a igualdade de precisões como hipótese nula e uma desigualdade estrita como alternativa. Também podemos considerar, para $j_{1}, j_{2} \in\{1, \ldots, r\}$, $\left|\alpha_{j_{1}}\right|=\left|\alpha_{j_{2}}\right|$ ou $\left|\beta_{j_{1}}-1\right|=\left|\beta_{j_{2}}-1\right|$ contra alternativas de desigualdade estrita. Estas hipóteses envolvem as distâncias dos vícios dos instrumentos $j_{1}$ e $j_{2}$ aos "vícios" do intrumento padrão e são de especial interesse pelo seguinte motivo: na situação altamente desejável em que $\alpha_{j_{1}}, \alpha_{j_{2}}$ são próximos de zero e $\beta_{j_{1}}, \beta_{j_{2}}$ são próximos de 1 , as distribuições das estatísticas para o teste de Wald tradicional e para o teste de 
distância não se comportam assintoticamente como esperado. A razão da dificuldade é que funções dos estimadores de máxima verossimilhança envolvidas nas estatísticas convergem em probabilidade para zero. Uma descrição detalhada do problema e uma sugestão para contorná-lo será apresentada.

Obteremos, primeiramente, os estimadores de máxima verossimilhança para os parâmetros no MRMC, um modelo mais geral que o de calibração comparativa, e provaremos a sua consistência e normalidade assintótica.

\subsection{Estimação de Máxima Verossimilhança no MRMC}

Consideremos o modelo dado em (4.1), (4.2) e (4.3), com $\Sigma_{\epsilon}=\sigma^{2} \Omega, \sigma^{2}>0$ desconhecido e $\Omega>0$ conhecida $^{2}$, que referenciaremos pela sigla MRMC. O modelo de calibração comparativa é um caso particular com $k=1$. Seja $\theta=\left(\mu_{x}, \alpha, \beta, \Sigma_{x}, \sigma^{2}\right)$. Então $\left\{Z_{i}, i \in \mathbb{N}\right\} \sim N I I D[\mu(\theta), \Sigma(\theta)]$, onde

$$
\begin{aligned}
\mu(\theta) & =\left(\begin{array}{c}
\mu_{x} \\
\alpha+\beta \mu_{x}
\end{array}\right) \\
\Sigma(\theta) & =\Sigma_{z}(\theta)+\sigma^{2} \Omega, \\
\Sigma_{z}(\theta) & =\left(\begin{array}{cc}
\Sigma_{x} & \Sigma_{x} \beta^{\prime} \\
\beta \Sigma_{x} & \beta \Sigma_{x} \beta^{\prime}
\end{array}\right) .
\end{aligned}
$$

O espaço paramétrico é dado por

$$
\Theta=\left\{\theta ; \mu_{x} \in \mathbb{R}^{k}, \alpha \in \mathbb{R}^{r}, \beta: r \times k, \Sigma_{x} \in \mathcal{M}_{k}, \sigma^{2}>0\right\} .
$$

Para encontrarmos o estimador de máxima verossimilhança para $\theta$ utilizaremos o mesmo método do capítulo 4 . O contradomínio da função $\theta \mapsto(\mu(\theta), \Sigma(\theta)), \theta \in \Theta$, está contido em $\mathbb{R}^{p} \times \Phi$, onde

$$
\Phi=\left\{\Sigma \in \mathcal{M}_{p} ; \quad \Sigma=\Sigma_{z}+\sigma^{2} \Omega, \operatorname{posto}\left(\Sigma_{z}\right)=k, \sigma^{2}>0\right\} .
$$

Seja $\left\{Z_{1}, \ldots, Z_{n}\right\} \sim N I I D(\mu, \Sigma)$, onde $\Sigma \in \Phi$. Seja $G$ a log-verossimilhança associada, dada por

$$
G(\mu, \Sigma)=-\frac{n}{2}\left[\log |\Sigma|+\operatorname{tr}\left(S_{Z} \Sigma^{-1}\right)+(\bar{Z}-\mu)^{\prime} \Sigma^{-1}(\bar{Z}-\mu)\right]
$$

\footnotetext{
${ }^{2}$ Esta é a diferença básica entre este modelo e o modelo definido no capítulo 4: no primeiro, a matriz de covariâncias dos erros de observação é conhecida a menos de uma constante e, no segundo, é estimada independentemente.
} 
para $(\mu, \Sigma) \in \mathbb{R}^{p} \times \Phi$. Fixando $\Sigma$, vemos que $G_{\Sigma}$ é maximizada em $\bar{Z}$. Assim, o próximo passo é maximizar

$$
G_{\bar{Z}}(\Sigma)=-\frac{n}{2}\left[\log |\Sigma|+\operatorname{tr}\left(S_{Z} \Sigma^{-1}\right)\right], \Sigma \in \Phi .
$$

Seja

$$
\hat{\Lambda}=\operatorname{diag}\left\{\hat{\lambda}_{1}, \ldots, \hat{\lambda}_{p}\right\}=\text { block } \operatorname{diag}\left\{\hat{\Lambda}_{(k)}, \hat{\Lambda}_{(r)}\right\},
$$

$\hat{\lambda}_{1} \geq \ldots \geq \hat{\lambda}_{p}, \hat{\Lambda}_{(k)}: k \times k$, a matriz de autovalores de $S_{Z}$ na métrica $\Omega$ e $T$ a matriz de autovetores associados. Consideremos a reparametrização

$$
\Sigma_{T}=T^{\prime} \Sigma T
$$

A menos de constantes aditivas, a log-verossimilhança reparametrizada é dada por

$$
G_{\bar{Z}}\left(\Sigma_{T}\right)=-\frac{n}{2}\left[\log \left|\Sigma_{T}\right|+\operatorname{tr}\left(\hat{\Lambda} \Sigma_{T}^{-1}\right)\right],
$$

$\Sigma_{T} \in \Phi_{T}$, onde

$$
\Phi_{T}=\left\{\Sigma_{T} \in \mathcal{M}_{p} ; \Sigma_{T}=\Sigma_{T z}+\sigma^{2} I, \operatorname{posto}\left(\Sigma_{T z}\right)=k, \sigma^{2}>0\right\} .
$$

Seja $\Gamma=\operatorname{diag}\left\{\gamma_{1}, \ldots, \gamma_{p}\right\}, \gamma_{1} \geq \ldots \geq \gamma_{p}$, a matriz de autovalores de $\Sigma_{T} \in \Phi_{T}$. Pelo lema 4.1.2,

$$
G_{\bar{Z}}(\Gamma) \geq G_{\bar{Z}}\left(\Sigma_{T}\right)
$$

Como $\Gamma \in \Phi_{T}$, isto significa que o máximo de $G_{\bar{Z}}$ está no conjunto

$$
\begin{aligned}
\Phi_{T}^{*}= & \left\{\Sigma_{T} \in \mathcal{M}_{p} ; \quad \Sigma_{T}=\operatorname{diag}\left\{\gamma_{1}, \ldots, \gamma_{p}\right\},\right. \\
& \left.\gamma_{i}=\eta_{i}+\sigma^{2}, \quad \eta_{1} \geq \ldots \geq \eta_{k}>0, \quad \eta_{k+1}, \ldots, \eta_{p}=0, \sigma^{2}>0\right\} .
\end{aligned}
$$

Seja $\Gamma \in \Phi_{T}^{*}, \Gamma=\operatorname{diag}\left\{\gamma_{1}, \ldots, \gamma_{p}\right\}$. Então

$$
\begin{aligned}
G_{\bar{Z}}(\Gamma) & =-\frac{n}{2}\left[\sum_{i=1}^{p} \log \gamma_{i}+\sum_{i=1}^{p} \hat{\lambda}_{i} \gamma_{i}^{-1}\right] \\
& =-\frac{n}{2}\left[\sum_{i=1}^{k} \log \left(\eta_{i}+\sigma^{2}\right)+\sum_{i=1}^{k} \hat{\lambda}_{i}\left(\eta_{i}+\sigma^{2}\right)^{-1}+r \log \sigma^{2}+\sum_{i=k+1}^{p} \hat{\lambda}_{i} \sigma^{-2}\right] .
\end{aligned}
$$

Fazendo $\eta=\left(\eta_{1}, \ldots, \eta_{k}\right)$ temos

$$
\frac{\partial G_{\bar{Z}}\left(\eta, \sigma^{2}\right)}{\partial \eta_{i}}=-\frac{n}{2}\left[\frac{1}{\eta_{i}+\sigma^{2}}-\frac{\hat{\lambda}_{i}}{\left(\eta_{i}+\sigma^{2}\right)^{2}}\right],
$$


$i=1, \ldots, k$,

$$
\frac{\partial G_{\bar{Z}}\left(\eta, \sigma^{2}\right)}{\partial \sigma^{2}}=-\frac{n}{2}\left[\sum_{i=1}^{k} \frac{1}{\eta_{i}+\sigma^{2}}-\sum_{i=1}^{k} \frac{\hat{\lambda}_{i}}{\left(\eta_{i}+\sigma^{2}\right)^{2}}\right]-\frac{n}{2}\left[\frac{r}{\sigma^{2}}-\sum_{i=k+1}^{p} \frac{\hat{\lambda}_{i}}{\sigma^{4}}\right] .
$$

Igualando a zero as derivadas em (5.3) e (5.4), obtemos um sistema em $\eta$ e $\sigma^{2}$ cuja solução é dada por $\hat{\eta}_{i}=\hat{\lambda}_{i}-\hat{\sigma}^{2}, i=1, \ldots, k \mathrm{e}$

$$
\hat{\sigma}^{2}=\frac{1}{r} \sum_{i=k+1}^{p} \hat{\lambda}_{i}
$$

Assim, o máximo de $G_{\bar{Z}}$ em $\Phi_{T}^{*}$ é atingido no ponto

$$
\hat{\Sigma}_{T}=\operatorname{diag}\left\{\hat{\gamma}_{1}, \ldots, \hat{\gamma}_{p}\right\}
$$

onde $\hat{\gamma}_{i}=\hat{\lambda}_{i}$, se $i=1, \ldots, k$ e $\hat{\gamma}_{i}=\hat{\sigma}^{2}$, se $i=k+1, \ldots, p$.

Seja $\hat{Q}$ uma matriz ortogonal de autovetores de $\Omega^{-1 / 2} S_{Z} \Omega^{-1 / 2}$ associada à matriz de autovalores $\hat{\Lambda}$. Particione $T$ como em (4.57) e $\hat{Q}$ de forma equivalente. O estimador para $\Sigma_{z}$ é dado por

$$
\begin{aligned}
\hat{\Sigma}_{z} & =\left(T^{\prime}\right)^{-1}\left(\hat{\Sigma}_{T}-\hat{\sigma}^{2} I\right) T^{-1} \\
& =\Omega T_{(k)}\left(\hat{\Lambda}_{(k)}-\hat{\sigma}^{2} I\right) T_{(k)}^{\prime} \Omega \\
& =\Omega^{1 / 2} \hat{Q}_{(k)}\left(\hat{\Lambda}_{(k)}-\hat{\sigma}^{2} I\right) \hat{Q}_{(k)}^{\prime} \Omega^{1 / 2}
\end{aligned}
$$

Os resultados da estimação estão sumarizados no enunciado do Teorema 5.2.1.

Teorema 5.2.1 Considere o Modelo de Regressão Linear Múltipla com Erros nas Variáveis definido por (4.1), (4.2) e (4.3), com $\Sigma_{\epsilon}=\sigma^{2} \Omega, \sigma^{2}>0$ e $\Omega>0$ conhecida. Sejam $\hat{\Lambda}=$ block $\operatorname{diag}\left\{\hat{\Lambda}_{(k)}, \hat{\Lambda}_{(r)}\right\}, \hat{\Lambda}_{(k)}: k \times k$, a matriz de autovalores de $S_{Z} n a$ métrica $\Omega$ e $T=\left(T_{(k)}, T_{(r)}\right), T_{(k)}: p \times k$ a matriz de autovetores associados. Os estimadores de máxima verossimilhança para os parâmetros do modelo são dados por

$$
\begin{aligned}
\hat{\mu}_{x} & =\bar{X} \\
\hat{\sigma}^{2} & =\frac{1}{r} \sum_{i=k+1}^{p} \hat{\lambda}_{i}, \\
\hat{\Sigma}_{z} & =\left(\begin{array}{ll}
\hat{\Sigma}_{x} & \hat{\Sigma}_{x y} \\
\hat{\Sigma}_{y x} & \hat{\Sigma}_{y}
\end{array}\right)=\Omega T_{(k)}\left(\hat{\Lambda}_{(k)}-\hat{\sigma}^{2} I\right) T_{(k)}^{\prime} \Omega, \\
\hat{\beta} & =\hat{\Sigma}_{y x} \hat{\Sigma}_{x}^{-1} \\
\hat{\alpha} & =\bar{Y}-\hat{\beta} \bar{X} .
\end{aligned}
$$




\subsection{Um Teste para a Qualidade do Ajuste}

Neste ponto podemos facilmente obter um teste de qualidade do ajuste para o MRMC. Seja $\left\{Z_{i}, i \in \mathbb{N}\right\} \sim N I I D(\mu, \Sigma), G$ a log-verossimilhança baseada em $n$ observações e considere as hipóteses

$$
H:(\mu, \Sigma)=(\mu(\theta), \Sigma(\theta)), \theta \in \Theta ; K:(\mu, \Sigma) \in \mathbb{R}^{p} \times \mathcal{M}_{p} .
$$

Seja $\zeta$ a estatística para o teste da razão de verossimilhança para $H$ contra $K$. $G$ é maximizada em $\left(\bar{Z}, S_{Z}\right)$. Por (5.2) e (5.6), temos

$$
\begin{aligned}
\sup G= & -\frac{n}{2}\left[\log |\hat{\Lambda}|+\operatorname{tr}\left(\hat{\Lambda} \hat{\Lambda}^{-1}\right)\right]=-\frac{n}{2}\left(\sum_{i=1}^{p} \log \hat{\lambda}_{i}+p\right), \\
\sup \{G(\mu(\theta), \Sigma(\theta)) ; \theta \in \Theta\}= & -\frac{n}{2}\left[\sum_{i=1}^{k} \log \hat{\lambda}_{i}+r \log \hat{\sigma}^{2}\right. \\
& \left.+\operatorname{tr}\left(\hat{\Lambda} \operatorname{block} \operatorname{diag}\left\{\hat{\Lambda}_{(k)}^{-1}, \hat{\sigma}^{-2} I_{r}\right\}\right)\right] \\
= & -\frac{n}{2}\left[\sum_{i=1}^{k} \log \hat{\lambda}_{i}+r \log \hat{\sigma}^{2}+k+\hat{\sigma}^{-2} \operatorname{tr}\left(\hat{\Lambda}_{(r)}\right)\right] \\
= & -\frac{n}{2}\left(\sum_{i=1}^{k} \log \hat{\lambda}_{i}+p+r \log \hat{\sigma}^{2}\right) .
\end{aligned}
$$

Assim, obtemos

$$
-2 \log \zeta=-n \sum_{i=k+1}^{p} \log \hat{\lambda}_{i}+n r \log \hat{\sigma}^{2} .
$$

$\mathrm{O}$ número de parâmetros quando $K$ é verdadeira é $p+(1 / 2) p(p+1)$. $\mathrm{O}$ número de parâmetros sob $H$ é $k+r+r k+(1 / 2) k(k+1)+1$. Pela teoria usual para o teste da razão de verossimilhança (Wilks, 1962, pg. 419), vem que, quando $H$ é verdadeira, a distribuição limite de $-2 \log \zeta$ é qui-quadrado com $(1 / 2) r(r+1)-1$ graus de liberdade.

\subsection{Consistência}

Com as hipóteses do Teorema 5.2.1 podemos provar facilmente que os estimadores de máxima verossimilhança são fortemente consistentes, bastando para isso seguir os passos da demonstração do teorema 4.3.1. Vamos provar somente que $\hat{\sigma}^{2}$ 
é consistente. Defina então $\Sigma_{\rho}, \Sigma_{\nu}$ e $\Lambda$ como em (4.33), (4.34) e (4.35), respectivamente, mas trocando $\Sigma_{\epsilon}$ por $\sigma^{2} \Omega$. Assim, $\lambda_{i}$ é autovalor de $\sigma^{-2} \Omega^{-1 / 2} \Sigma \Omega^{-1 / 2}$, $i=1, \ldots, p$, onde $\Sigma=\Sigma(\theta)$, etc. Temos então que $\Lambda_{(r)}=I$ e $\Lambda_{(k)}-I$ é matriz de autovalores de $\Sigma_{\rho}^{-1 / 2} \Sigma_{x} \Sigma_{\rho}^{-1 / 2}$. A matriz de autovalores de $\Omega^{-1 / 2} \Sigma \Omega^{-1 / 2}$ é $\sigma^{2} \Lambda$. Como $\Omega^{-1 / 2} S_{Z} \Omega^{-1 / 2} \stackrel{\mathrm{qc}}{\longrightarrow} \Omega^{-1 / 2} \Sigma \Omega^{-1 / 2}$, vem que $\hat{\Lambda} \stackrel{\mathrm{qc}}{\longrightarrow} \sigma^{2} \Lambda \mathrm{e}$, portanto, para $\theta \in \Theta$

$$
\hat{\Lambda}_{(r)} \stackrel{\mathrm{qc}(\theta)}{\longrightarrow} \sigma^{2} I
$$

Assim,

$$
\hat{\sigma}^{2}=\frac{1}{r} \operatorname{tr}\left(\hat{\Lambda}_{(r)}\right) \stackrel{\mathrm{qc}(\theta)}{\longrightarrow} \frac{1}{r} \sigma^{2} \operatorname{tr}\left(I_{r}\right)=\sigma^{2} .
$$

\subsection{Normalidade Assintótica}

A normalidade assintótica da sequência de estimadores

$$
\left.\left\{n^{1 / 2}\left[(\hat{\alpha}-\alpha)^{\prime},(\operatorname{vec}(\hat{\beta}-\beta))^{\prime},\left(\operatorname{vech}\left(\hat{\Sigma}_{x}-\Sigma_{x}\right)\right)^{\prime}, \hat{\sigma}^{2}-\sigma^{2}\right)\right]^{\prime}\right\}
$$

pode ser demonstrada com argumentos semelhantes aos utilizados na demonstração do teorema 4.4.1. A idéia é escrever o $n$-ésimo vetor desta sequência como função do $n$-ésimo elemento da sequência no Lema 4.4.1 mais um componente que converge em probabilidade para zero.

Defina $P_{(k)}, F_{k}$ e $F_{r}$ como em (4.58), (4.65) e (4.66), respectivamente, com $\Omega$ no lugar de $S$. Fixe $\theta \in \Theta$. Temos então

$$
\begin{array}{rll}
P_{k k} \hat{\Lambda}_{(k)} P_{k k}^{\prime} & \stackrel{\mathrm{qc}(\theta)}{\longrightarrow} \Sigma_{x}+\Sigma_{\rho} ; \\
T_{(r)} T_{(r)}^{\prime} & \stackrel{\mathrm{qc}(\theta)}{\longrightarrow} \sigma^{2}(-\beta, I)^{\prime} \Sigma_{\nu}^{-1}(-\beta, I) ; \\
T_{r r} T_{r r}^{\prime} & \stackrel{\mathrm{qc}(\theta)}{\longrightarrow} \sigma^{2} \Sigma_{\nu}^{-1} ; \\
T_{r r} \hat{\Lambda}_{(r)} T_{r r}^{\prime} & \stackrel{\mathrm{qc}(\theta)}{\longrightarrow} \sigma^{4} \Sigma_{\nu}^{-1} ; \\
I_{r} \otimes F_{k}+F_{r}^{\prime} \otimes I_{k} & \stackrel{\mathrm{qc}(\theta)}{\longrightarrow} \sigma^{2} I_{r} \otimes\left(\Sigma_{\rho}^{-1} \Sigma_{x}\right) .
\end{array}
$$

Podemos provar que

$$
\begin{aligned}
\operatorname{vec}(\hat{\beta}-\beta)^{\prime}= & \left(I_{r} \otimes F_{k}+F_{r}^{\prime} \otimes I_{k}\right)^{-1} \\
& \times\left\{\left[\sigma^{2}(-\beta, I) \otimes \Sigma_{\rho}^{-1}\right] \operatorname{vec}\left(S_{x \epsilon}\right)+\left[(-\beta, I) \otimes\left(I, \beta^{\prime}\right) \Omega^{-1}\right] \operatorname{vec}\left(S_{\epsilon}-\sigma^{2} \Omega\right)\right\} .
\end{aligned}
$$


Em conjunto com (5.12), isto implica em

$$
\begin{aligned}
\operatorname{vec}(\hat{\beta}-\beta)^{\prime}= & {\left[(-\beta, I) \otimes \Sigma_{x}^{-1}\right] \operatorname{vec}\left(S_{x \epsilon}\right)+\left[(-\beta, I) \otimes \Sigma_{x}^{-1} \Sigma_{\rho}\left(I, \beta^{\prime}\right) \sigma^{-2} \Omega^{-1}\right] \operatorname{vec}\left(S_{\epsilon}-\sigma^{2} \Omega\right) } \\
& +o_{p}\left(n^{-1 / 2}\right) .
\end{aligned}
$$

A normalidade assintótica de (5.7) será obtida a partir desta última fórmula, mais as fórmulas (5.14), (5.15), (5.19) e o Lema 4.4.1.

Temos que

$$
\begin{aligned}
n^{1 / 2}(\hat{\alpha}-\alpha) & =n^{1 / 2}(\bar{Y}-\hat{\beta} \bar{X}-\alpha) \\
& =n^{1 / 2}[\beta \bar{x}+\bar{e}-\hat{\beta}(\bar{x}+\bar{\delta})] \\
& =n^{1 / 2}[(\beta-\hat{\beta}) \bar{x}+\bar{e}-\beta \bar{\delta}]+n^{1 / 2}(\beta-\hat{\beta}) \bar{\delta} \\
& =n^{1 / 2}(\beta-\hat{\beta}) \bar{x}+n^{1 / 2}(-\beta, I) \bar{\epsilon}+o_{p}(1) \\
& =n^{1 / 2}(\beta-\hat{\beta}) \mu_{x}+n^{1 / 2}(-\beta, I) \bar{\epsilon}+o_{p}(1) .
\end{aligned}
$$

Também

$$
\hat{\sigma}^{2}-\sigma^{2}=r^{-1} \operatorname{tr}\left(\hat{\Lambda}_{(r)}-\sigma^{2} I\right) .
$$

Observe que $(-\hat{\beta}, I)^{\prime} T_{r r}=T_{(r)}($ ver 4.64$)$ e portanto

$$
\begin{aligned}
\hat{\Lambda}_{(r)}= & T_{(r)}^{\prime}\left(S_{Z}-\Sigma\right) T_{(r)}+T_{(r)}^{\prime} \Sigma T_{(r)} \\
= & T_{(r)}^{\prime}\left[\left(I, \beta^{\prime}\right)^{\prime}\left(S_{x}-\Sigma_{x}\right)\left(I, \beta^{\prime}\right)+S_{\epsilon x}\left(I, \beta^{\prime}\right)+\left(I, \beta^{\prime}\right)^{\prime} S_{x \epsilon}+\left(S_{\epsilon}-\sigma^{2} \Omega\right)\right] T_{(r)} \\
& \quad+T_{(r)}^{\prime}\left[\left(I, \beta^{\prime}\right)^{\prime} \Sigma_{x}\left(I, \beta^{\prime}\right)+\sigma^{2} \Omega\right] T_{(r)} \\
= & T_{r r}^{\prime}(\hat{\beta}-\beta)\left(S_{x}-\Sigma_{x}\right)(\hat{\beta}-\beta)^{\prime} T_{r r}+T_{(r)}^{\prime} S_{\epsilon x}\left(I, \beta^{\prime}\right) T_{(r)} \\
& +T_{(r)}^{\prime}\left(I, \beta^{\prime}\right)^{\prime} S_{x \epsilon} T_{(r)}+T_{(r)}^{\prime}\left(S_{\epsilon}-\sigma^{2} \Omega\right) T_{(r)}+T_{r r}^{\prime}(\hat{\beta}-\beta) \Sigma_{x}(\hat{\beta}-\beta)^{\prime} T_{r r}+\sigma^{2} T_{(r)}^{\prime} \Omega T_{(r)} .
\end{aligned}
$$

A segunda igualdade vale por (4.69). Como $T_{(r)}^{\prime} \Omega T_{(r)}=I$, temos

$$
\begin{aligned}
\operatorname{tr}\left(\hat{\Lambda}_{(r)}-\sigma^{2} I\right)= & \operatorname{tr}\left[T_{r r} T_{r r}^{\prime}(\hat{\beta}-\beta)\left(S_{x}-\Sigma_{x}\right)(\hat{\beta}-\beta)^{\prime}\right]+\operatorname{tr}\left[\left(I, \beta^{\prime}\right) T_{(r)} T_{(r)}^{\prime} S_{\epsilon x}\right] \\
& +\operatorname{tr}\left[T_{(r)} T_{(r)}^{\prime}\left(I, \beta^{\prime}\right)^{\prime} S_{x \epsilon}\right]+\operatorname{tr}\left[T_{(r)} T_{(r)}^{\prime}\left(S_{\epsilon}-\sigma^{2} \Omega\right)\right] \\
& +\operatorname{tr}\left[T_{r r} T_{r r}^{\prime}(\hat{\beta}-\beta) \Sigma_{x}(\hat{\beta}-\beta)^{\prime}\right] .
\end{aligned}
$$

Agora, $n^{1 / 2}\left(S_{x}-\Sigma_{x}\right)$ converge em distribuição, $\hat{\beta}-\beta \stackrel{\text { qc }(\theta)}{\longrightarrow} 0$ e $T_{r r} T_{r r}^{\prime}$ converge quase 
certamente. Por (5.9), vem que

$$
\begin{aligned}
\operatorname{tr}\left(\hat{\Lambda}_{(r)}-\sigma^{2} I\right)= & \operatorname{tr}\left[\sigma^{2}\left(I, \beta^{\prime}\right)(-\beta, I)^{\prime} \Sigma_{\nu}^{-1}(-\beta, I) S_{\epsilon x}\right]+\operatorname{tr}\left[\sigma^{2}(-\beta, I)^{\prime} \Sigma_{\nu}^{-1}(-\beta, I)\left(I, \beta^{\prime}\right)^{\prime} S_{x \epsilon}\right] \\
& +\operatorname{tr}\left[\sigma^{2}(-\beta, I)^{\prime} \Sigma_{\nu}^{-1}(-\beta, I)\left(S_{\epsilon}-\sigma^{2} \Omega\right)\right]+o_{p}\left(n^{-1 / 2}\right) \\
= & \operatorname{tr}\left[\sigma^{2}(-\beta, I)^{\prime} \Sigma_{\nu}^{-1}(-\beta, I)\left(S_{\epsilon}-\sigma^{2} \Omega\right)\right]+o_{p}\left(n^{-1 / 2}\right) \\
= & \left\{\operatorname{vec}\left[\sigma^{2}(-\beta, I)^{\prime} \Sigma_{\nu}^{-1}(-\beta, I)\right]\right\}^{\prime} \operatorname{vec}\left(S_{\epsilon}-\sigma^{2} \Omega\right)+o_{p}\left(n^{-1 / 2}\right) \\
= & \left\{\sigma^{2}\left[\operatorname{vec}\left(\Sigma_{\nu}^{-1}\right)\right]^{\prime}[(-\beta, I) \otimes(-\beta, I)]\right\} \operatorname{vec}\left(S_{\epsilon}-\sigma^{2} \Omega\right)+o_{p}\left(n^{-1 / 2}\right) .
\end{aligned}
$$

A partir da relação $S_{Z}=\Omega T \hat{\Lambda} T^{\prime} \Omega$ obtemos, somando e subtraindo $\hat{\sigma}^{2} \Omega T_{(k)} T_{(k)}^{\prime} \Omega$,

$$
\begin{aligned}
S_{Z} & =\Omega T_{(k)}\left(\hat{\Lambda}_{(k)}-\hat{\sigma}^{2} I\right) T_{(k)}^{\prime} \Omega+\Omega T_{(k)} T_{(k)}^{\prime} \hat{\sigma}^{2} \Omega+\Omega\left(T_{(r)} \hat{\Lambda}_{(r)} T_{(r)}^{\prime}\right) \Omega \\
& =\hat{\Sigma}_{z}+\hat{\sigma}^{2} \Omega\left[T_{(k)} T_{(k)}^{\prime}+T_{(r)} T_{(r)}^{\prime}\right] \Omega+\Omega T_{(r)}\left(\hat{\Lambda}_{(r)}-\hat{\sigma}^{2} I\right) T_{(r)}^{\prime} \Omega \\
& =\hat{\Sigma}_{z}+\hat{\sigma}^{2} \Omega T T^{\prime} \Omega+\Omega T_{(r)}\left(\hat{\Lambda}_{(r)}-\hat{\sigma}^{2} I\right) T_{(r)}^{\prime} \Omega \\
& =\hat{\Sigma}_{z}+\hat{\sigma}^{2} \Omega+\Omega(-\hat{\beta}, I)^{\prime} T_{r r}\left(\hat{\Lambda}_{(r)}-\hat{\sigma}^{2} I\right) T_{r r}^{\prime}(-\hat{\beta}, I) \Omega
\end{aligned}
$$

onde a terceira igualdade é justificada pela relação $\Omega T=\left(T^{-1}\right)^{\prime}$. Temos também que

$$
\begin{aligned}
(-\beta, I) S_{Z}(-\hat{\beta}, I)^{\prime} T_{r r} & =(-\beta, I) S_{Z} T_{(r)}=(-\beta, I) \Omega T_{(r)} \hat{\Lambda}_{(r)} \\
& =(-\beta, I) \Omega(-\hat{\beta}, I)^{\prime} T_{r r} \hat{\Lambda}_{(r)}
\end{aligned}
$$

e, portanto,

$$
\begin{aligned}
& T_{r r} \hat{\Lambda}_{(r)} T_{r r}^{\prime}-\hat{\sigma}^{2} T_{r r} T_{r r}^{\prime} \\
&=\left[(-\beta, I) \Omega(-\hat{\beta}, I)^{\prime}\right]^{-1}\left[(-\beta, I) S_{Z}(-\hat{\beta}, I)^{\prime} T_{r r} T_{r r}^{\prime}\right]-\hat{\sigma}^{2} T_{r r} T_{r r}^{\prime} \\
&=\left[(-\beta, I) \Omega(-\hat{\beta}, I)^{\prime}\right]^{-1} \\
& \quad \times\left\{(-\beta, I)\left[\left(I, \beta^{\prime}\right)^{\prime} S_{x}\left(I, \beta^{\prime}\right)+S_{\epsilon x}\left(I, \beta^{\prime}\right)+\left(I, \beta^{\prime}\right)^{\prime} S_{x \epsilon}+S_{\epsilon}\right](-\hat{\beta}, I)^{\prime} T_{r r} T_{r r}^{\prime}\right\}-\hat{\sigma}^{2} T_{r r} T_{r r}^{\prime} \\
&=\left[(-\beta, I) \Omega(-\hat{\beta}, I)^{\prime}\right]^{-1} \\
& \\
& \quad \times\left[(-\beta, I) S_{\epsilon x}(\beta-\hat{\beta})^{\prime} T_{r r} T_{r r}^{\prime}+(-\beta, I) S_{\epsilon}(-\hat{\beta}, I)^{\prime} T_{r r} T_{r r}^{\prime}\right]-\hat{\sigma}^{2} T_{r r} T_{r r}^{\prime} \\
&= {\left[(-\beta, I) \Omega(-\hat{\beta}, I)^{\prime}\right]^{-1}(-\beta, I) S_{\epsilon x}(\beta-\hat{\beta})^{\prime} T_{r r} T_{r r}^{\prime} } \\
&+\left[(-\beta, I) \Omega(-\hat{\beta}, I)^{\prime}\right]^{-1}\left[(-\beta, I)\left(S_{\epsilon}-\hat{\sigma}^{2} \Omega\right)(-\hat{\beta}, I)^{\prime} T_{r r} T_{r r}^{\prime}+(-\beta, I) \hat{\sigma}^{2} \Omega(-\hat{\beta}, I)^{\prime} T_{r r} T_{r r}^{\prime}\right] \\
&-\hat{\sigma}^{2} T_{r r} T_{r r}^{\prime} \\
&=\left\{(-\beta, I) \Omega(-\hat{\beta}, I)^{\prime}\right]^{-1}(-\beta, I)\left(S_{\epsilon}-\hat{\sigma}^{2} \Omega\right)(-\hat{\beta}, I)^{\prime} T_{r r} T_{r r}^{\prime}+o_{p}\left(n^{-1 / 2}\right),
\end{aligned}
$$

ou seja,

$$
T_{r r}\left(\hat{\Lambda}_{(r)}-\hat{\sigma}^{2} I\right) T_{r r}^{\prime}=\sigma^{4} \Sigma_{\nu}^{-1}(-\beta, I)\left(S_{\epsilon}-\hat{\sigma}^{2} \Omega\right)(-\beta, I)^{\prime} \Sigma_{\nu}^{-1}+o_{p}\left(n^{-1 / 2}\right) .
$$


Isto é obtido substituindo em (5.17) as relações (5.10),

$$
(-\hat{\beta}, I)^{\prime}=(-\beta, I)^{\prime}+o_{p}(1),
$$

$\mathrm{e}$

$$
\left[(-\beta, I) \Omega(-\hat{\beta}, I)^{\prime}\right]^{-1}=\left[(-\beta, I) \Omega(-\beta, I)^{\prime}\right]^{-1}+o_{p}(1)=\sigma^{2} \Sigma_{\nu}^{-1}+o_{p}(1) .
$$

Substituindo (5.18) em (5.16), temos

$$
\begin{aligned}
S_{Z}= & \hat{\Sigma}_{z}+\hat{\sigma}^{2} \Omega+\sigma^{4} \Omega(-\hat{\beta}, I)^{\prime} \Sigma_{\nu}^{-1}(-\beta, I)\left(S_{\epsilon}-\hat{\sigma}^{2} \Omega\right)(-\hat{\beta}, I)^{\prime} \Sigma_{\nu}^{-1}(-\hat{\beta}, I) \Omega \\
& +o_{p}\left(n^{-1 / 2}\right) .
\end{aligned}
$$

Mas, por (5.13),

$$
\begin{aligned}
\Omega(-\hat{\beta}, I)^{\prime} & =\Omega(-\hat{\beta}, I)^{\prime}+\Omega(-\beta, I)^{\prime}-\Omega(-\beta, I)^{\prime} \\
& =\Omega(\beta-\hat{\beta}, 0)^{\prime}+\Omega(-\beta, I)^{\prime} \\
& =\Omega(-\beta, I)^{\prime}+O_{p}\left(n^{-1 / 2}\right)
\end{aligned}
$$

e, portanto,

$$
\begin{aligned}
S_{Z}= & \hat{\Sigma}_{z}+\hat{\sigma}^{2} \Omega+\sigma^{4}\left[\Omega(-\beta, I)^{\prime}+O_{p}\left(n^{-1 / 2}\right)\right] \Sigma_{\nu}^{-1}(-\beta, I)\left(S_{\epsilon}-\hat{\sigma}^{2} \Omega\right)(-\beta, I)^{\prime} \Sigma_{\nu}^{-1} \\
& \times\left[(-\beta, I) \Omega+O_{p}\left(n^{-1 / 2}\right)\right] \\
= & \hat{\Sigma}_{z}+\hat{\sigma}^{2} \Omega+\sigma^{4} \Omega(-\beta, I)^{\prime} \Sigma_{\nu}^{-1}(-\beta, I)\left(S_{\epsilon}-\hat{\sigma}^{2} \Omega\right)(-\beta, I)^{\prime} \Sigma_{\nu}^{-1}(-\beta, I) \Omega+o_{p}\left(n^{-1 / 2}\right) .
\end{aligned}
$$

Seja

$$
\Sigma_{\nu \epsilon}=\sigma^{2}(-\beta, I) \Omega, \Sigma_{\epsilon \nu}=\Sigma_{\nu \epsilon}^{\prime}
$$

Então*

$$
\begin{aligned}
S_{Z}= & \hat{\Sigma}_{z}+\hat{\sigma}^{2} \Omega+\sigma^{2} \Sigma_{\epsilon \nu} \Sigma_{\nu}^{-1}\left[(-\beta, I)\left(S_{\epsilon}-\sigma^{2} \Omega\right)(-\beta, I)^{\prime}+(-\beta, I)\left(\sigma^{2} \Omega-\hat{\sigma}^{2} \Omega\right)(-\beta, I)^{\prime}\right] \\
& \times \sigma^{-2} \Sigma_{\nu}^{-1} \Sigma_{\nu \epsilon}+o_{p}\left(n^{-1 / 2}\right) .
\end{aligned}
$$

Seja $\Omega_{k k}$ a submatriz superior à esquerda de ordem $k \times k \operatorname{em} \Omega$ e escreva $\Sigma_{\epsilon \nu}=\left(\Sigma_{\delta \nu}^{\prime}, \cdot\right)$.

Temos que

$$
\begin{aligned}
S_{X}= & \hat{\Sigma}_{x}+\hat{\sigma}^{2} \Omega_{k k}+\Sigma_{\delta \nu} \Sigma_{\nu}^{-1}(-\beta, I)\left(S_{\epsilon}-\sigma^{2} \Omega\right)(-\beta, I)^{\prime} \Sigma_{\nu}^{-1} \Sigma_{\nu \delta} \\
& +\Sigma_{\delta \nu} \Sigma_{\nu}^{-1}(-\beta, I)\left(\sigma^{2}-\hat{\sigma}^{2}\right) \Omega(-\beta, I)^{\prime} \Sigma_{\nu}^{-1} \Sigma_{\nu \delta}+o_{p}\left(n^{-1 / 2}\right) .
\end{aligned}
$$

Isto implica em

$$
\begin{aligned}
\hat{\Sigma}_{x}-\Sigma_{x}= & S_{X}-\Sigma_{X}+\left(\sigma^{2}-\hat{\sigma}^{2}\right) \Omega_{k k}-\Sigma_{\delta \nu} \Sigma_{\nu}^{-1}(-\beta, I)\left(S_{\epsilon}-\sigma^{2} \Omega\right)(-\beta, I)^{\prime} \Sigma_{\nu}^{-1} \Sigma_{\nu \delta} \\
& -\Sigma_{\delta \nu} \Sigma_{\nu}^{-1}(-\beta, I)\left(\sigma^{2}-\hat{\sigma}^{2}\right) \Omega(-\beta, I)^{\prime} \Sigma_{\nu}^{-1} \Sigma_{\nu \delta}+o_{p}\left(n^{-1 / 2}\right),
\end{aligned}
$$


onde $\Sigma_{X}$ é a matriz de covariâncias de $X_{1}$. Assim,

$$
\begin{aligned}
\operatorname{vec}\left(\hat{\Sigma}_{x}-\Sigma_{x}\right)= & \operatorname{vec}\left(S_{X}-\Sigma_{X}\right)+\left(\sigma^{2}-\hat{\sigma}^{2}\right) \operatorname{vec}\left(\Omega_{k k}\right) \\
& -\left[\Sigma_{\delta \nu} \Sigma_{\nu}^{-1}(-\beta, I) \otimes \Sigma_{\delta \nu} \Sigma_{\nu}^{-1}(-\beta, I)\right] \operatorname{vec}\left(S_{\epsilon}-\sigma^{2} \Omega\right) \\
& -\left[\Sigma_{\delta \nu} \Sigma_{\nu}^{-1}(-\beta, I) \otimes \Sigma_{\delta \nu} \Sigma_{\nu}^{-1}(-\beta, I)\right]\left(\sigma^{2}-\hat{\sigma}^{2}\right) \operatorname{vec}(\Omega) \\
& +o_{p}\left(n^{-1 / 2}\right)
\end{aligned}
$$

Usando estes resultados podemos facilmente obter a matriz de covariâncias da distribuição limite de (5.7). Os blocos desta matriz serão denotados por $\Omega_{\alpha}$ para a parte referente à variância assintótica de $\left\{n^{1 / 2}(\hat{\alpha}-\alpha)\right\}, \Omega_{\alpha \beta}$ para a parte referente à covariância assintótica entre $\left\{n^{1 / 2}(\hat{\alpha}-\alpha)\right\}$ e $\left\{n^{1 / 2} \operatorname{vec}(\hat{\beta}-\beta)\right\}$, etc. Comecemos por $\Omega_{\beta}$. Procedendo como na demonstração do teorema 4.4 .1 e utilizando (5.13), temos

$$
\Omega_{\beta}=\Sigma_{x}^{-1} \otimes \Sigma_{\nu}+\Sigma_{x}^{-1} \Sigma_{p} \Sigma_{x}^{-1} \otimes \Sigma_{\nu}
$$

Pelo Teorema Central do Limite

$$
n^{1 / 2}(-\beta, I) \bar{\epsilon} \stackrel{\mathcal{D}(\theta)}{\longrightarrow} N\left(0, \Sigma_{\nu}\right) .
$$

Pela normalidade de $\left\{\left(x_{i}^{\prime}, \epsilon_{i}^{\prime}\right)^{\prime}, i=1, \ldots, n\right\}$, temos que a matriz de covariâncias amostral

$$
\left(\begin{array}{ll}
S_{x} & S_{x \epsilon} \\
S_{\epsilon x} & S_{\epsilon}
\end{array}\right)
$$

é independente de $\bar{\epsilon}$. Desta forma, por (5.14) e (5.13),

$$
\begin{aligned}
\Omega_{\alpha} & =\Sigma_{\nu}+\left(\mu_{x}^{\prime} \otimes I_{r}\right) \Omega_{\beta}\left(\mu_{x} \otimes I_{r}\right) . \\
\Omega_{\alpha \beta} & =-\left(\mu_{x}^{\prime} \otimes I_{r}\right) \Omega_{\beta} .
\end{aligned}
$$

Estas são as covariâncias que nos interessam para a formulação de testes para as hipóteses restritas no caso do modelo de calibração linear. As demais covariâncias podem ser obtidas após algumas (poucas) manipulações algébricas, usando as fórmulas (5.14), (5.15), (5.19) e o Lema 4.4.1. Os resultados são apresentados no Teorema 5.5.1.

Teorema 5.5.1 Considere os estimadores de máxima verossimilhança para o MRMC 
apresentados no teorema 5.2.1. Então, para todo $\theta \in \Theta$,

$$
\begin{aligned}
& \left.n^{1 / 2}\left[(\hat{\alpha}-\hat{\alpha})^{\prime},(\operatorname{vec}(\hat{\beta}-\beta))^{\prime},\left(\operatorname{vech}\left(\hat{\Sigma}_{x}-\Sigma_{x}\right)\right)^{\prime}, \hat{\sigma}^{2}-\sigma^{2}\right)\right]^{\prime} \stackrel{\mathcal{D}(\theta)}{\longrightarrow} \\
& \left.\left(\begin{array}{llll}
\Omega_{\alpha} & \Omega_{\alpha \beta} & \Omega_{\alpha x} & 0 \\
\Omega_{\beta \alpha}^{\prime} & \Omega_{\beta} & \Omega_{\beta x} & 0 \\
\Omega_{\alpha x}^{\prime} & \Omega_{\beta x}^{\prime} & \Omega_{x} & \Omega_{x \sigma} \\
0 & 0 & \Omega_{x \sigma}^{\prime} & \Omega_{\sigma}
\end{array}\right)\right),
\end{aligned}
$$

onde

$$
\begin{aligned}
\Omega_{\alpha}= & \Sigma_{\nu}+\left(\mu_{x}^{\prime} \otimes I_{r}\right) \Omega_{\beta}\left(\mu_{x} \otimes I_{r}\right) \\
\Omega_{x}= & 2 \Delta_{k}\left[\Sigma_{X} \otimes \Sigma_{X}-\Sigma_{\delta \nu} \Sigma_{\nu}^{-1} \Sigma_{\nu \delta} \otimes \Sigma_{\delta \nu} \Sigma_{\nu}^{-1} \Sigma_{\nu \delta}\right] \Delta_{k}^{\prime} ; \\
& +2 r^{-1} \operatorname{vech}\left(\sigma^{2} \Omega_{k k}-\Sigma_{\delta \nu} \Sigma_{\nu}^{-1} \Sigma_{\nu \delta}\right) \operatorname{vech}^{\prime}\left(\sigma^{2} \Omega_{k k}-\Sigma_{\delta \nu} \Sigma_{\nu}^{-1} \Sigma_{\nu \delta}\right) \\
\Omega_{\sigma}= & 2 r^{-1} \sigma^{4} \\
\Omega_{\beta}= & \Sigma_{x}^{-1} \otimes \Sigma_{\nu}+\Sigma_{x}^{-1} \Sigma_{\rho} \Sigma_{x}^{-1} \otimes \Sigma_{\nu} \\
\Omega_{\alpha \beta}= & -\left(\mu_{x}^{\prime} \otimes I_{r}\right) \Omega_{\beta} \\
\Omega_{\alpha x}= & -\left(\mu_{x}^{\prime} \otimes I_{r}\right) \Omega_{\beta x} \\
\Omega_{\beta x}= & 2\left[\left(I_{k}+\Sigma_{\rho} \Sigma_{x}^{-1}\right) \otimes \Sigma_{\delta \nu}\right] \Delta_{k}^{\prime} \\
\Omega_{x \sigma}= & -2 r^{-1} \sigma^{2} \operatorname{vech}\left(\sigma^{2} \Omega_{k k}-\Sigma_{\delta \nu} \Sigma_{\delta \nu}^{-1} \Sigma_{\nu \delta}\right) .
\end{aligned}
$$

\subsection{Dificuldades de Aplicação dos Testes de Wald e de Distância e um Teste de Distância Modi- ficado}

Retomemos os tópicos e a notação do capítulo 2. Para testar as hipóteses em (2.5), tanto o Teste de Wald quanto o Teste de Distância baseiam-se na convergência dada em (2.7). Por exemplo, no caso da estatística de Distância, este resultado implica em (2.10), que por sua vez define a distribuição limite da estatística sob a hipótese nula. Evidentemente, é crucial que tenhamos $\Sigma(\theta)>0$ para que isto ocorra, já que a variável aleatória do lado direito de (2.10) sequer é definida caso tenhamos o determinante desta matriz igual a zero. Assim, para uma correta aplicação do teste, $J(\theta)$ deve necessariamente ter posto completo. Infelizmente isto não ocorre quando 
desejamos testar algumas hipóteses importantes envolvendo parâmetros do MRMC. Com algumas simples modificações na estatística de Wald, Lütkepohl \& Burda (1997) mostram como, mesmo sob estas condições desfavoráveis, pode-se obter a almejada distribuição nula assintótica qui-quadrado. Baseados em suas idéias, apresentaremos a seguir uma adaptação do Teste de Distância que pode ser útil na presença destas anomalias. O método será aplicado ao MRMC, mas as idéias podem ser facilmente adaptadas a outros modelos e hipóteses não considerados aqui.

Para exemplificar, considere o MRMC $\operatorname{com} k=1$ e $r=2$ e as hipóteses

$$
H_{\alpha}:\left|\alpha_{1}\right|=\left|\alpha_{2}\right|, K_{\alpha}:\left|\alpha_{1}\right|>\left|\alpha_{2}\right|
$$

$H_{\alpha}$ significa que os instrumentos 1 e 2 têm, em magnitude, vícios aditivos iguais. Denotemos o estimador de máxima verossimilhança para $\theta$ por $\hat{\theta}$. Pelo teorema 5.5.1 vem que, para $\theta \in H_{\alpha}$,

$$
\hat{\gamma}=n^{1 / 2}\left(\hat{\alpha}_{1}^{2}-\hat{\alpha}_{2}^{2}\right) \stackrel{\mathcal{D}(\theta)}{\longrightarrow} N\left(0, \sigma_{\alpha}^{2}(\theta)\right),
$$

onde

$$
\begin{aligned}
\sigma_{\alpha}^{2}(\theta) & =4\left(\alpha_{1},-\alpha_{2}\right) \Omega_{\alpha}\left(\alpha_{1},-\alpha_{2}\right)^{\prime} \\
& =4\left(\sigma^{2}+\frac{\mu_{x}^{2} \sigma^{2}}{\sigma_{x}^{2}}+\frac{\mu_{x}^{2} \sigma^{4}}{\sigma_{x}^{4}}\right)\left[\left(\alpha_{1} \beta_{1}-\alpha_{2} \beta_{2}\right)^{2}+\alpha_{1}^{2}+\alpha_{2}^{2}\right]
\end{aligned}
$$

Temos que $\sigma_{\alpha}^{2}(\theta)>0$ se e somente se $\alpha \neq 0$. A estatística de distância para testar $H_{\alpha}$ contra $K_{\alpha}$,

$$
\mathbf{D}=\frac{\hat{\gamma}^{2}}{\sigma_{\alpha}^{2}(\hat{\theta})} I_{(0, \infty)}(\hat{\gamma}),
$$

tem distribuição limite $F$ dada em (3.16), quando $\theta \in H_{\alpha}$ e $\alpha \neq 0$. Como veremos em experimentos com dados simulados, isto não é necessariamente verdadeiro quando temos $\alpha=0$. Mesmo quando $\alpha \neq 0$ podemos ter problemas em relação à aproximação à $F$. Isto ocorre, por exemplo, quando $\theta \in H_{\alpha}$ e $\alpha_{1}$ e $\alpha_{2}$ são pequenos.

Apresentaremos agora uma teoria geral que pode ser útil para contornar dificuldades deste tipo. Será feita uma modificação na estatística de distância, de modo a garantir a sua convergência em distribuição a uma mistura de funções de distribuição qui-quadrado, quando a hipótese nula for verdadeira.

Considere então as hipóteses definidas em (2.5),

$$
H: h(\theta)=0, K: h(\theta) \in \mathcal{A} .
$$


Temos que, para $\theta \in \Theta$,

$$
n^{1 / 2}[h(\bar{\theta})-h(\theta)] \stackrel{\mathcal{D}(\theta)}{\longrightarrow} N\left(0, J(\theta) \Omega(\theta) J(\theta)^{\prime}\right),
$$

mesmo quando $J(\theta)$ não for de posto completo e/ou $\Omega(\theta)$ tiver determinante nulo. Seja $\left\{R(\theta) \sim N\left(0, \Sigma_{R}(\theta)\right), \theta \in \Theta\right\}$ um processo estocástico independente de $\{\bar{\theta}\}$, escolhido de tal maneira que $\Sigma_{R}(\theta)+\Sigma(\theta)$ é positiva definida, $\Sigma(\theta)=J(\theta) \Omega(\theta) J(\theta)^{\prime}$. Assumimos que a função $\theta \mapsto \Sigma_{R}(\theta), \theta \in \Theta$ é contínua. Defina

$$
\bar{\gamma}=n^{1 / 2} h(\bar{\theta})+R(\bar{\theta}),
$$

e considere a estatística de distância modificada

$$
\mathbf{D}^{M}=\left\|\pi_{\bar{\Sigma}_{\gamma}}(\bar{\gamma}, \mathcal{A})\right\|_{\bar{\Sigma}_{\gamma}}^{2}
$$

onde

$$
\bar{\Sigma}_{\gamma}=\Sigma_{\gamma}(\bar{\theta})=\Sigma(\bar{\theta})+\Sigma_{R}(\bar{\theta}) .
$$

Para $\theta \in H$, a distribuição limite desta estatística é uma mistura de funções de distribuição qui-quadrado. A demonstração deste resultado é consequência do próximo lema. Os objetos aleatórios que aparecem no enunciado estão todos definidos no mesmo espaço de probabilidade.

Lema 5.6.1 Seja $\phi$ uma função contínua definida em $\mathcal{S}$, um subconjunto do espaço Euclideano, com $\phi(s)$ sendo uma matriz simétrica e não-negativa definida, para todo $s \in \mathcal{S}$. Seja $\left\{W_{t}, t \in \mathcal{S}\right\}$ um processo estocástico tal que $W_{t} \sim N(0, \phi(t))$ para todo $t \in \mathcal{S}, e\left\{T_{n}\right\}$ uma sequência de vetores aleatórios em $\mathcal{S}$ independente de $\left\{W_{t}\right\}$ e tal que $T_{n} \stackrel{q c}{\longrightarrow} t_{0}, t_{0} \in \mathcal{S}$. Então

(i) $W_{T_{n}}$ e $T_{n}$ são independentes, $n \in \mathbb{N}$;

(ii) $W_{T_{n}} \stackrel{\mathcal{D}}{\longrightarrow} N\left(0, \phi\left(t_{0}\right)\right)$

Demonstração: (i) $E$ denotará esperanças. Temos que, para $A$ e $B$ eventos quaisquer,

$$
P\left(W_{T_{n}} \in A, T_{n} \in B\right)=E\left[P\left(W_{T_{n}} \in A, T_{n} \in B \mid T_{n}\right)\right]
$$

Mas

$$
\begin{aligned}
P\left(W_{T_{n}} \in A, T_{n} \in B \mid T_{n}=t_{n}\right) & =P\left(W_{t_{n}} \in A \mid T_{n}=t_{n}\right) I_{\left(t_{n} \in B\right)} \\
& =P\left(W_{t_{n}} \in A\right) I_{\left(t_{n} \in B\right)},
\end{aligned}
$$


pela independência entre $W_{t_{n}}$ e $T_{n}$. Tomando esperanças, obtemos $(i)$.

(ii) Seja $\psi_{W_{T_{n}}}$ a função característica de $W_{T_{n}}$. Devemos mostrar que, para cada $u$,

$$
\psi_{W_{T_{n}}}(u) \rightarrow \exp \left\{-\frac{1}{2} u^{\prime} \phi\left(t_{0}\right) u\right\}
$$

quando $n \rightarrow \infty$. Temos que

$$
\psi_{W_{T_{n}}}(u)=E\left\{E\left[\exp \left(i u^{\prime} W_{T_{n}}\right) \mid T_{n}\right]\right\}
$$

Mas

$$
\begin{aligned}
E\left[\exp \left(i u^{\prime} W_{T_{n}}\right) \mid T_{n}=t_{n}\right] & =E\left[\exp \left(i u^{\prime} W_{t_{n}}\right) \mid T_{n}=t_{n}\right]=E\left[\exp \left(i u^{\prime} W_{t_{n}}\right)\right] \\
& =\exp \left\{-\frac{1}{2} u^{\prime} \phi\left(t_{n}\right) u\right\}
\end{aligned}
$$

pela independência entre $W_{t_{n}}$ e $T_{n}$. Assim,

$$
E\left[\exp \left(i u^{\prime} W_{T_{n}}\right) \mid T_{n}\right]=\exp \left\{-\frac{1}{2} u^{\prime} \phi\left(T_{n}\right) u\right\}
$$

e portanto

$$
E\left[\exp \left(i u^{\prime} W_{T_{n}}\right) \mid T_{n}\right] \stackrel{\text { qc }}{\longrightarrow} \exp \left\{-\frac{1}{2} u^{\prime} \phi\left(t_{0}\right) u\right\} .
$$

Como $\left|\exp \left\{-\frac{1}{2} u^{\prime} \phi\left(T_{n}\right) u\right\}\right| \leq 1$ para todo $u$ e todo $n \in \mathbb{N}$, o resultado segue pelo Teorema da Convergência Dominada.

Teorema 5.6.1 Seja $\theta \in H$. Então, a distribuição limite de $\mathbf{D}^{M}$ é dada em (2.18), $\operatorname{com} \Sigma_{\gamma}(\theta)$ no lugar de $\Sigma(\theta)$.

Demonstração: Imediata. A partir de (5.23) e do Lema 5.6.1, temos que (5.24) converge em distribuição para uma $N\left(0, \Sigma(\theta)+\Sigma_{R}(\theta)\right)$. Então, é só utilizar os argumentos que levaram à demonstração do teorema 2.2.2.

A função $\Sigma_{R}$ deve ser estipulada tendo-se em mente dois objetivos: (i) garantir a convergência apresentada no Teorema 5.6 .1 para todo $\theta \in H$ e (ii) manter as propriedades de poder do Teste de Distância para alternativas "distantes" de pontos $\theta$ para os quais $|\Sigma(\theta)|=0$. Como a escolha de $\Sigma_{R}$ depende da particular forma de $\Sigma$, cada situação de modelo/hipóteses deve ser considerada em separado.

Para exemplificar, vamos mostrar como formular um teste de distância modificado para as hipóteses em (5.20). Seja $R(\theta) \sim N\left(0, \sigma_{R}^{2}(\theta)\right)$ independente de $\hat{\theta}$. Definindo

$$
\hat{\gamma}=n^{1 / 2}\left(\hat{\alpha}_{1}^{2}-\hat{\alpha}_{2}^{2}\right)+R(\hat{\theta})
$$


temos que

$$
\mathrm{D}^{M}=\frac{\hat{\gamma}^{2}}{\sigma_{\alpha}^{2}(\hat{\theta})+\sigma_{R}^{2}(\hat{\theta})} I_{(0, \infty)}(\hat{\gamma}),
$$

onde $\sigma_{\alpha}^{2}(\hat{\theta})$ está definida em (5.22). Uma possibilidade de escolha de $\sigma_{R}^{2}$ que não cumpre a contento o objetivo (ii), como veremos nos estudos de simulação (tabela 5.1), é a função constante. Uma outra sugestão é considerar a função

$$
\sigma_{R}^{2}(\theta)=\left(\sigma_{*}^{2}-\sigma_{\alpha}^{2}(\theta)\right) I_{(0, \infty)}\left(\sigma_{*}^{2}-\sigma_{\alpha}^{2}(\theta)\right),
$$

onde $\sigma_{*}^{2}>0$. A idéia é não utilizar a correção $R(\hat{\theta})$ quando $\sigma_{\alpha}^{2}(\hat{\theta})$ for suficientemente grande.

A seguir apresentamos alguns estudos de simulação. Cada elemento nas tabelas 5.1 - 5.5 corresponde à taxa de rejeição de $H_{\alpha}$ em 2000 amostras do MRMC, simuladas de acordo com os parâmetros especificados, para os testes de Wald, Distância, um teste de Wald modificado sugerido por Lütkepohl \& Burda (1997) e o teste de distância modificado. A estatística do teste de Wald modificado é dada por

$$
\frac{\hat{\gamma}^{2}}{\sigma_{\alpha}^{2}(\hat{\theta})+\sigma_{R}^{2}(\hat{\theta})},
$$

que, pela demonstração do teorema 5.6.1, tem distribuição limite qui-quadrado com 1 grau de liberdade sob $H_{\alpha}$. Tomamos sempre $\Omega=I, \alpha_{2}=0.05, \mu_{x}=2, \beta_{1}=1.2 \mathrm{e}$ $\beta_{2}=1$. Na tabela 5.1 temos $\sigma_{R}^{2}=0.3$ para todo $\theta$, nas demais usamos (5.26).

Estas tabelas mostram que, mesmo para amostras grandes $(n=500)$, as estatísticas de Wald e Distância não têm uma distribuição próxima de uma quiquadrado com 1 grau de liberdade e $F$ (ver 3.16), respectivamente, quando $\theta \in H_{\alpha}$ e $\alpha_{1}$ e $\alpha_{2}$ são pequenos. Pode-se observar que as taxas de rejeição sob $H_{\alpha}$ são mais próximas dos níveis nominais $(5 \%$ e $10 \%)$ quando utilizamos os testes modificados. Apesar das evidentes melhoras em relação aos testes usuais, esta aproximação não chega a ser satisfatória quando tomamos amostras pequenas e $\sigma_{x}^{2}$ e $\sigma^{2}$ "grandes". Para grandes valores de $\alpha_{1}$ praticamente não há perda de poder em relação ao Teste de Distância, quando utilizamos (5.26). Isto pode ser verificado nas tabelas 5.2 5.5. No entanto, há claras perdas quando tomamos $\sigma_{R}^{2}$ constante $=0.3$, compare a tabela 5.1 com a terceira e a quarta colunas do primeiro bloco (nível=5\%) da tabela 5.2 .

Analogamente, podemos definir um Teste de Distância Modificado para testar

$$
H_{\beta}:\left|\beta_{1}-1\right|=\left|\beta_{2}-1\right| \text { contra } K_{\beta}:\left|\beta_{1}-1\right|>\left|\beta_{2}-1\right| \text {. }
$$


Tabela 5.1: Taxas de Rejeição para os Testes de Wald, Distância, Wald Modificado e Distância Modificado $\left(H_{\alpha}:\left|\alpha_{1}\right|=\left|\alpha_{2}\right|, K_{\alpha}:\left|\alpha_{1}\right|>\left|\alpha_{2}\right| ; \sigma_{x}^{2}=0.1, \sigma^{2}=0.01\right.$, $\sigma_{R}^{2}=0.3, n=100$, nível $\left.=5 \%\right)$

\begin{tabular}{lcc}
\hline$\alpha_{1}$ & \multicolumn{2}{c}{ Teste } \\
& Wald Mod. & Distância Mod. \\
\hline 0.05 & 0.0420 & 0.0475 \\
0.10 & 0.0470 & 0.0675 \\
0.15 & 0.0500 & 0.0810 \\
0.20 & 0.0595 & 0.1070 \\
0.25 & 0.0925 & 0.1605 \\
0.30 & 0.1650 & 0.2750 \\
0.35 & 0.2400 & 0.3760 \\
0.40 & 0.3425 & 0.4890 \\
\hline
\end{tabular}

Sob $H_{\beta}$, temos

$$
n^{1 / 2}\left[\left(\hat{\beta}_{1}-1\right)^{2}-\left(\hat{\beta}_{2}-1\right)^{2}\right] \stackrel{\mathcal{D}}{\longrightarrow} N\left(0, \sigma_{\beta}^{2}(\theta)\right),
$$

onde

$\sigma_{\beta}^{2}(\theta)=4\left(\frac{\sigma^{2}}{\sigma_{x}^{2}}+\frac{\sigma^{4}}{\sigma_{x}^{4}\left(1+\beta^{\prime} \beta\right)}\right)\left\{\left[\beta_{1}\left(\beta_{1}-1\right)-\beta_{2}\left(\beta_{2}-1\right)\right]^{2}+\left(\beta_{1}-1\right)^{2}+\left(\beta_{2}-1\right)^{2}\right\}$.

(5.28), (5.29), (5.25) e (5.26), com $\sigma_{\beta}^{2}(\theta)$ no lugar de $\sigma_{\alpha}^{2}(\theta)$, definem a estatística de teste. Nas tabelas 5.6 e 5.7 temos um estudo de poder por simulações. Fixamos $\beta_{2}=0.95, \alpha_{1}=0.4, \alpha_{2}=0.01, \mu_{x}=2, \sigma_{x}^{2}=0.1, \sigma^{2}=0.01$.

Diferentemente do que ocorre em relação aos testes para as hipóteses com o vício aditivo, neste caso parece que temos uma aproximação satisfatória as distribuições nulas limite também para pequenas amostras.

Finalmente, apresentamos na tabela 5.8 uma comparação entre taxas de rejeição dos testes de Wald e Distância para as hipóteses

$$
H: \beta_{1}^{2}=\beta_{2}^{2}=1, K: 1 \geq \beta_{1}^{2} \geq \beta_{2}^{2} .
$$

Se vale $H$, os três instrumentos têm a mesma precisão. Em $K$ temos uma ordem na precisão dos instrumentos, o instrumento 2 não é mais preciso que o 1 , e o 1 
Tabela 5.2: Taxas de Rejeição para os Testes de Wald, Distância, Wald Modificado e Distância Modificado $\left(H_{\alpha}:\left|\alpha_{1}\right|=\left|\alpha_{2}\right|, K_{\alpha}:\left|\alpha_{1}\right|>\left|\alpha_{2}\right| ; \sigma_{x}^{2}=0.1, \sigma^{2}=0.01\right.$, $n=100)$

\begin{tabular}{ccccccccccc}
\hline Nível & $\alpha_{1}$ & Wald & Distância & \multicolumn{3}{c}{ Wald Mod. } & \multicolumn{3}{c}{ Dist. Mod. } \\
\hline \multirow{2}{*}{$5 \%$} & & & & $\sigma_{*}^{2}$ & 0.1 & 0.2 & 0.3 & 0.1 & 0.2 & 0.3 \\
\cline { 6 - 11 } & 0.05 & 0.0005 & 0.0020 & & 0.0210 & 0.0320 & 0.0390 & 0.0205 & 0.0315 & 0.0375 \\
& 0.10 & 0.0010 & 0.0050 & & 0.0135 & 0.0260 & 0.0395 & 0.0225 & 0.0445 & 0.0530 \\
& 0.15 & 0.0115 & 0.0325 & & 0.0200 & 0.0380 & 0.0440 & 0.0500 & 0.0725 & 0.0795 \\
& 0.20 & 0.0315 & 0.1030 & & 0.0375 & 0.0495 & 0.0625 & 0.1105 & 0.1325 & 0.1405 \\
& 0.25 & 0.1040 & 0.2180 & & 0.1060 & 0.1100 & 0.1205 & 0.2205 & 0.2380 & 0.2460 \\
& 0.30 & 0.1900 & 0.3570 & & 0.1910 & 0.1930 & 0.2015 & 0.3580 & 0.3615 & 0.3710 \\
& 0.35 & 0.3575 & 0.5295 & & 0.3575 & 0.3580 & 0.3625 & 0.5295 & 0.5315 & 0.5355 \\
& 0.40 & 0.5050 & 0.6990 & & 0.5050 & 0.5060 & 0.5080 & 0.6990 & 0.6995 & 0.6990 \\
\hline $10 \%$ & 0.05 & 0.0040 & 0.0125 & & 0.0505 & 0.0760 & 0.0870 & 0.0685 & 0.0910 & 0.0950 \\
& 0.10 & 0.0110 & 0.0430 & & 0.0485 & 0.0725 & 0.0875 & 0.0905 & 0.1170 & 0.1215 \\
& 0.15 & 0.0370 & 0.1255 & & 0.0605 & 0.0995 & 0.1155 & 0.1605 & 0.1970 & 0.1910 \\
& 0.20 & 0.0930 & 0.2305 & & 0.1085 & 0.1375 & 0.1550 & 0.2500 & 0.2885 & 0.2785 \\
& 0.25 & 0.2030 & 0.4050 & & 0.2065 & 0.2280 & 0.2505 & 0.4135 & 0.4320 & 0.4100 \\
& 0.30 & 0.3445 & 0.5665 & & 0.3470 & 0.3540 & 0.3615 & 0.5705 & 0.5780 & 0.5530 \\
& 0.35 & 0.5410 & 0.7275 & & 0.5415 & 0.5445 & 0.5515 & 0.7285 & 0.7305 & 0.7260 \\
& 0.40 & 0.6920 & 0.8375 & & 0.6920 & 0.6920 & 0.6915 & 0.8375 & 0.8400 & 0.8345 \\
\hline
\end{tabular}

não é mais preciso que o padrão. Problemas de aproximação à distribuição nula limite também podem acorrer nas proximidades de um $\theta \operatorname{com} \beta_{1}=0$ ou $\beta_{2}=0$, mas acreditamos que precisões tão pequenas não ocorrem com frequência na prática. Fixamos $\beta_{1}=1, \alpha_{1}=0.4, \alpha_{2}=0.01, \mu_{x}=2, \sigma_{x}^{2}=0.1, \sigma^{2}=0.01$.

\subsection{Uma Aplicação}

A tabela 5.9 apresenta medições de volume do testículo esquerdo em 40 adolescentes $^{3}$. Cinco métodos de medição foram utilizados mas, para efeito deste exemplo,

${ }^{3}$ Agradecemos à Prof $^{a}$ Daisy G.S. Tu por gentilmente nos ter cedido estes dados. 
Tabela 5.3: Taxas de Rejeição para os Testes de Wald, Distância, Wald Modificado e Distância Modificado $\left(H_{\alpha}:\left|\alpha_{1}\right|=\left|\alpha_{2}\right|, K_{\alpha}:\left|\alpha_{1}\right|>\left|\alpha_{2}\right| ; \sigma_{x}^{2}=0.1, \sigma^{2}=0.01\right.$, $n=500$ )

\begin{tabular}{|c|c|c|c|c|c|c|c|c|c|c|}
\hline Nível & $\alpha_{1}$ & Wald & Distância & & & Tald Moc & & & ist. $\mathrm{N}$ & \\
\hline \multirow{9}{*}{$5 \%$} & & & & $\sigma_{*}^{2}$ & 0.1 & 0.2 & 0.3 & 0.1 & 0.2 & 0.3 \\
\hline & 0.05 & 0.0025 & 0.0035 & & 0.0460 & 0.0495 & 0.0515 & 0.0535 & 0.0535 & 0.0535 \\
\hline & 0.10 & 0.0255 & 0.0945 & & 0.0790 & 0.0720 & 0.0690 & 0.1400 & 0.1085 & 0.0990 \\
\hline & 0.15 & 0.2490 & 0.4425 & & 0.3010 & 0.1760 & 0.1315 & 0.4440 & 0.2850 & 0.2150 \\
\hline & 0.20 & 0.6665 & 0.8365 & & 95 & 0.4910 & 0.3455 & 0.8190 & 0.6115 & 0.4690 \\
\hline & 0.2 & 0.9030 & 0.9 & & 0.9035 & 0.8300 & 0.6890 & 0.9590 & 0.8880 & 0.7835 \\
\hline & 0.3 & 0.9835 & 0.9 & & 9835 & 0.9780 & 0.9340 & 0.9960 & 0.9925 & 0.9640 \\
\hline & 0.35 & 0.9975 & 1. & & 9975 & 0.9970 & 0.9915 & 1.0000 & 0.9995 & 0.9985 \\
\hline & 0.40 & 0.9995 & 1.0000 & & 0.9995 & 0.9995 & 0.9995 & 1.0000 & 1.0000 & 1.0000 \\
\hline \multirow[t]{8}{*}{$10 \%$} & 0.05 & 0.0070 & 0.01 & & 0.0935 & 0.0960 & 0.1005 & 0.1005 & 0.1005 & 0.0990 \\
\hline & 0.10 & 0.0795 & 0.2255 & & 0.1480 & 0.1245 & 0.1165 & 0.2330 & 0.1880 & 0.1660 \\
\hline & 0.15 & 0.4540 & 0.6 & & 0.4480 & 0.2765 & 0.2105 & 0.5900 & 0.4010 & 0.3275 \\
\hline & 0.20 & 0.8230 & & & 0.8065 & 0.6135 & 0.4760 & 0.8960 & 0.7300 & 0.6175 \\
\hline & 0.25 & 0.9675 & 0.9 & & 0.9670 & 0.9135 & 0.8055 & 0.9885 & 0.9545 & 0.8905 \\
\hline & 0.30 & 0.9955 & 0.9985 & & 0.9955 & 0.9860 & 0.9645 & 0.9985 & 0.9955 & 0.9800 \\
\hline & 0. & 0.9990 & 1.0000 & & 0.9990 & 0.9980 & 0.9970 & 1.0000 & 0.9995 & 0.9990 \\
\hline & 0.40 & 1.0000 & 1.0000 & & 1.0000 & 1.0000 & 1.0000 & 1.0000 & 1.0000 & 1.0000 \\
\hline
\end{tabular}

usaremos somente a parte referente a três métodos: Ultrasom (tomado como método padrão), um método gráfico proposto pelos autores do estudo ("instrumento" 1) e "Ring orchidometer" ("instrumento" 2). A análise a seguir é feita usando o MRMC com $\Omega=I$, após uma transformação dos dados por raiz cúbica, a fim de garantir normalidade. Efetuamos o teste de qualidade do ajuste para o MRMC, conforme o descrito na seção 5.3, obtendo uma probabilidade de significância de 0.0721 . Em Chopkevitch et al. (1996) encontramos uma análise utilizando o teste de Wald usual, mas levando em conta a possibilidade de variâncias diferentes para os erros de mensuração. Os valores das estimativas dos parâmetros e das variâncias assintóticas dos parâmetros de interesse estão na tabela 5.10. 
Tabela 5.4: Taxas de Rejeição para os Testes de Wald, Distância, Wald Modificado e Distância Modificado $\left(H_{\alpha}:\left|\alpha_{1}\right|=\left|\alpha_{2}\right|, K_{\alpha}:\left|\alpha_{1}\right|>\left|\alpha_{2}\right| ; \sigma_{x}^{2}=0.4, \sigma^{2}=0.1\right.$, $n=100$ )

\begin{tabular}{|c|c|c|c|c|c|c|c|c|c|c|}
\hline Nível & $\alpha_{1}$ & Wald & Distância & & & Vald Mo & & & ist. Mo & \\
\hline \multirow{9}{*}{$5 \%$} & & & & $\sigma_{*}^{2}$ & 0.3 & 0.4 & 0.5 & 0.3 & 0.4 & 0.5 \\
\hline & 0.05 & 0.0010 & 0.0015 & & 0.0110 & 0.0145 & 0.0175 & 0.0145 & 0.0205 & 0.0250 \\
\hline & 0.10 & 0.0015 & 0.0060 & & 0.0085 & 0.0115 & 0.0130 & 0.0135 & 0.0165 & 0.0200 \\
\hline & 0.15 & 0.0020 & 0.0085 & & 0.0095 & 0.0125 & 0.0145 & 0.0160 & 0.0185 & 0.0230 \\
\hline & 0.20 & 0.0115 & 0.0325 & & 0.0160 & 0.0165 & 0.0180 & 0.0370 & 0.0405 & 0.0410 \\
\hline & 0.25 & 0.0200 & 0.0550 & & 0.0215 & 0.0235 & 0.0260 & 0.0600 & 0.0635 & 0.0640 \\
\hline & 0.30 & 0.0320 & 0.0835 & & 0.0330 & 0.0330 & 0.0350 & 0.0845 & 0.0850 & 0.0865 \\
\hline & 0.35 & 0.0550 & 0.1330 & & 0.0555 & 0.0555 & 0.0560 & 0.1340 & 0.1350 & 0.1355 \\
\hline & 0.40 & 0.1040 & 0.2110 & & 0.1040 & 0.1040 & 0.1040 & 0.2115 & 0.2115 & 0.2120 \\
\hline \multirow[t]{8}{*}{$10 \%$} & 0.05 & 0.0025 & 0.0075 & & 0.0235 & 0.0320 & 0.0370 & 0.0335 & 0.0405 & 0.0445 \\
\hline & 0.10 & 0.0055 & 0.0210 & & 0.0290 & 0.0375 & 0.0450 & 0.0445 & 0.0505 & 0.0605 \\
\hline & 0.15 & 0.0090 & 0.0425 & & 0.0235 & 0.0275 & 0.0340 & 0.0600 & 0.0695 & 0.0770 \\
\hline & 0.20 & 0.0275 & 0.0975 & & 0.0350 & 0.0380 & 0.0410 & 0.1085 & 0.1150 & 0.1215 \\
\hline & 0.25 & 0.0455 & 0.1450 & & 0.0510 & 0.0525 & 0.0540 & 0.1490 & 0.1535 & 0.1600 \\
\hline & 0.30 & 0.0925 & 0.2075 & & 0.0965 & 0.0975 & 0.1000 & 0.2140 & 0.2170 & 0.2195 \\
\hline & 0.35 & 0.1310 & 0.2810 & & 0.1340 & 0.1365 & 0.1390 & 0.2860 & 0.2875 & 0.2925 \\
\hline & 0.40 & 0.1945 . & 0.3865 & & 0.1965 & 0.1965 & 0.1970 & 0.3875 & 0.3880 & 0.3885 \\
\hline
\end{tabular}

Vamos testar primeiramente as hipóteses em (5.30), já que é natural supor que as medições feitas por Ultrasom são mais precisas. A probabilidade de significância usando o teste de distância é 0.0283 , indicando a rejeição de $H$. No entanto, a probabilidade de significância para o teste de Wald é 0.0911 e não rejeitaríamos $H$ em um teste de nível $5 \%$.

Em relação aos métodos 1 e 2, as estimativas na tabela 5.10 indicam que o vício aditivo do método 2 é, em magnitude, maior que o do método 1 . As estimativas para os vícios multiplicativos são bem próximas. Considere então as hipóteses $H_{\alpha}$, dada em (5.20), e $H: \beta_{1}=\beta_{2}$. Vamos testá-las seqüencialmente, usando $\mathbf{D}^{M}$ para testar $H_{\alpha}$ e o teste de Wald para $H$. Um procedimento adequado é o sugerido por 
Tabela 5.5: Taxas de Rejeição para os Testes de Wald, Distância, Wald Modificado e Distância Modificado $\left(H_{\alpha}:\left|\alpha_{1}\right|=\left|\alpha_{2}\right|, K_{\alpha}:\left|\alpha_{1}\right|>\left|\alpha_{2}\right| ; \sigma_{x}^{2}=0.4, \sigma^{2}=0.1\right.$, $n=500$ )

\begin{tabular}{ccccccccccc}
\hline Nível & $\alpha_{1}$ & Wald & Distância & \multicolumn{3}{c}{ Wald Mod } & \multicolumn{3}{c}{ Dist. Mod. } \\
\hline \multirow{3}{*}{$5 \%$} & 0.05 & 0.0015 & 0.0030 & & 0.0280 & 0.0370 & 0.0405 & 0.0355 & 0.0400 & 0.0420 \\
& 0.10 & 0.0035 & 0.0210 & & 0.0260 & 0.0345 & 0.0395 & 0.0605 & 0.0725 & 0.0780 \\
& 0.15 & 0.0245 & 0.0910 & & 0.0390 & 0.0495 & 0.0630 & 0.1205 & 0.1400 & 0.1465 \\
& 0.20 & 0.1140 & 0.2365 & & 0.1170 & 0.1215 & 0.1320 & 0.2460 & 0.2620 & 0.2795 \\
& 0.25 & 0.2875 & 0.4875 & & 0.2880 & 0.2915 & 0.2995 & 0.4920 & 0.5000 & 0.5115 \\
& 0.30 & 0.5125 & 0.6955 & & 0.5125 & 0.5130 & 0.5135 & 0.6960 & 0.6985 & 0.7050 \\
& 0.35 & 0.7185 & 0.8425 & & 0.7185 & 0.7185 & 0.7185 & 0.8425 & 0.8425 & 0.8425 \\
& 0.40 & 0.8580 & 0.9320 & & 0.8580 & 0.8580 & 0.8580 & 0.9320 & 0.9320 & 0.9320 \\
\hline $10 \%$ & 0.05 & 0.0050 & 0.0140 & & 0.0625 & 0.0760 & 0.0785 & 0.0895 & 0.0950 & 0.0965 \\
& 0.10 & 0.0165 & 0.0770 & & 0.0725 & 0.0880 & 0.0930 & 0.1505 & 0.1505 & 0.1400 \\
& 0.15 & 0.0925 & 0.2430 & & 0.1315 & 0.1540 & 0.1660 & 0.3130 & 0.3075 & 0.2860 \\
& 0.20 & 0.2580 & 0.4835 & & 0.2755 & 0.2970 & 0.3180 & 0.5245 & 0.5255 & 0.5090 \\
& 0.25 & 0.4740 & 0.6920 & & 0.4785 & 0.4865 & 0.4990 & 0.7045 & 0.7070 & 0.6880 \\
& 0.30 & 0.6980 & 0.8520 & & 0.6990 & 0.7025 & 0.7090 & 0.8545 & 0.8580 & 0.8515 \\
& 0.35 & 0.8605 & 0.9400 & & 0.8605 & 0.8615 & 0.8625 & 0.9405 & 0.9405 & 0.9405 \\
& 0.40 & 0.9340 & 0.9715 & & 0.9340 & 0.9340 & 0.9340 & 0.9715 & 0.9715 & 0.9710 \\
\hline
\end{tabular}

Holm (1979). A aplicação deste procedimento é extremamente simples e, no caso de duas hipóteses, pode ser descrita da seguinte maneira: primeiramente ordenamos as probabilidades de significância obtidas efetuando-se cada um dos testes, obtendo, digamos, $p^{(1)} \leq p^{(2)}$. Denote as hipóteses associadas por $H^{(1)}$ e $H^{(2)}$, respectivamente. Seja $\xi \in(0,1)$. Execute os seguintes passos:

Passo 1: Se $p^{(1)}>\xi / 2$ aceite $H^{(1)}$ e $H^{(2)}$ e pare; caso contrário, rejeite $H^{(1)}$ e continue; Passo 2: Se $p^{(2)}>\xi$ aceite $H^{(2)}$; caso contrário, rejeite $H^{(2)}$.

$\mathrm{O}$ procedimento garante um nível de significância múltiplo ${ }^{4}$ igual a $\xi$.

\footnotetext{
${ }^{4}$ Segundo Holm (1979): "O procedimento sequencial com regiões críticas $C_{1}, \ldots, C_{n}$ para testar $H_{1}, \ldots, H_{n}$ é dito ter nível de significância múltiplo $\xi$ se para cada conjunto de índices não vazio
} 
Tabela 5.6: Taxas de Rejeição para os Testes de Wald, Distância, Wald Modificado e Distância Modificado $\left(H_{\beta}:\left|\beta_{1}-1\right|=\left|\beta_{2}-1\right|, K_{\beta}:\left|\beta_{1}-1\right|>\left|\beta_{2}-1\right|, n=50\right)$

\begin{tabular}{ccccccccccc}
\hline Nível & $\beta_{1}$ & Wald & Distância & \multicolumn{3}{c}{ Wald Mod } & \multicolumn{3}{c}{ Dist. Mod. } \\
\hline \multirow{3}{*}{$5 \%$} & 1.05 & 0.0065 & 0.0040 & & 0.0365 & 0.0430 & 0.0485 & 0.0400 & 0.0445 & 0.0430 \\
& 1.10 & 0.0045 & 0.0195 & & 0.0380 & 0.0495 & 0.0560 & 0.0605 & 0.0720 & 0.0715 \\
& 1.15 & 0.0240 & 0.0850 & & 0.0430 & 0.0520 & 0.0495 & 0.1155 & 0.1050 & 0.0850 \\
& 1.20 & 0.1010 & 0.2175 & & 0.1080 & 0.1105 & 0.0795 & 0.2255 & 0.2130 & 0.1490 \\
& 1.25 & 0.2200 & 0.4010 & & 0.2275 & 0.2240 & 0.1550 & 0.4055 & 0.3695 & 0.2640 \\
& 1.30 & 0.3815 & 0.5700 & & 0.3825 & 0.3730 & 0.2745 & 0.5700 & 0.5445 & 0.4100 \\
& 1.35 & 0.6020 & 0.7700 & & 0.6020 & 0.5920 & 0.4895 & 0.7710 & 0.7540 & 0.6340 \\
& 1.40 & 0.7405 & 0.8645 & & 0.7400 & 0.7360 & 0.6750 & 0.8630 & 0.8580 & 0.7865 \\
\hline $10 \%$ & 1.05 & 0.0155 & 0.0245 & & 0.0740 & 0.0860 & 0.0915 & 0.0915 & 0.1000 & 0.1005 \\
& 1.10 & 0.0300 & 0.0860 & & 0.0935 & 0.1075 & 0.1080 & 0.1440 & 0.1355 & 0.1290 \\
& 1.15 & 0.0820 & 0.2355 & & 0.1270 & 0.1265 & 0.1125 & 0.2505 & 0.2255 & 0.1815 \\
& 1.20 & 0.2330 & 0.4295 & & 0.2425 & 0.2225 & 0.1720 & 0.4320 & 0.3680 & 0.2620 \\
& 1.25 & 0.4020 & 0.6000 & & 0.4115 & 0.3785 & 0.2760 & 0.6015 & 0.5465 & 0.4075 \\
& 1.30 & 0.5735 & 0.7585 & & 0.5750 & 0.5450 & 0.4250 & 0.7540 & 0.7100 & 0.5670 \\
& 1.35 & 0.7410 & 0.8745 & & 0.7415 & 0.7250 & 0.6205 & 0.8760 & 0.8555 & 0.7385 \\
& 1.40 & 0.8525 & 0.9400 & & 0.8525 & 0.8485 & 0.7715 & 0.9395 & 0.9350 & 0.8715 \\
\hline
\end{tabular}

Obtemos $\sigma_{\alpha}^{2}(\hat{\theta})=0.2506$ e probabilidades de significância iguais a 0.0268 para o teste de distância modificado ( $\operatorname{com} \sigma_{*}^{2}=0.1$ ) e 0.3650 para o teste de Wald. Se usarmos um nível de significância múltiplo de pelo menos $6 \%$, decidimos pela rejeição de $H_{\alpha}$ e aceitação de $H$, indicando que, apesar dos métodos serem igualmente precisos, o método gráfico é menos viciado que o "Ring Orchidometer". Para comparar, efetuamos um teste de Wald para a interseção de $H_{\alpha}$ e $H$ (a hipótese $\left|\alpha_{1}\right|=\left|\alpha_{2}\right|, \beta_{1}=\beta_{2}$ ) e obtivemos a probabilidade de significância 0.1547 , indicando a sua aceitação.

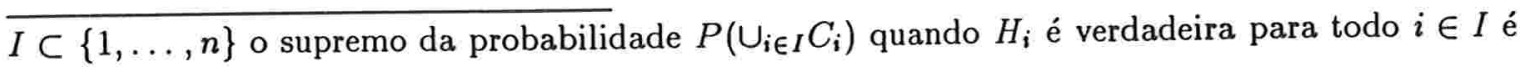
menor ou igual a $\xi "$ 
Tabela 5.7: Taxas de Rejeição para os Testes de Wald, Distância, Wald Modificado e Distância Modificado $\left(H_{\beta}:\left|\beta_{1}-1\right|=\left|\beta_{2}-1\right|, K_{\beta}:\left|\beta_{1}-1\right|>\left|\beta_{2}-1\right|, n=100\right)$

\begin{tabular}{ccccccccccc}
\hline Nível & $\beta_{1}$ & Wald & Distância & \multicolumn{3}{c}{ Wald Mod } & \multicolumn{3}{c}{ Dist. Mod. } \\
\hline \multirow{3}{*}{$5 \%$} & 1.05 & 0.0045 & 0.0040 & & 0.0445 & 0.0500 & 0.0550 & 0.0530 & 0.0585 & 0.0585 \\
& 1.10 & 0.0200 & 0.0640 & & 0.0560 & 0.0535 & 0.0530 & 0.0940 & 0.0830 & 0.0765 \\
& 1.15 & 0.1205 & 0.2605 & & 0.1395 & 0.1055 & 0.0795 & 0.2460 & 0.1720 & 0.1250 \\
& 1.20 & 0.3255 & 0.5105 & & 0.3185 & 0.2325 & 0.1355 & 0.4865 & 0.3370 & 0.2215 \\
& 1.25 & 0.5765 & 0.7240 & & 0.5690 & 0.4660 & 0.2740 & 0.7105 & 0.5925 & 0.3890 \\
& 1.30 & 0.8030 & 0.9005 & & 0.8020 & 0.7450 & 0.5075 & 0.8995 & 0.8440 & 0.6445 \\
& 1.35 & 0.9285 & 0.9745 & & 0.9285 & 0.9195 & 0.7885 & 0.9745 & 0.9665 & 0.8645 \\
& 1.40 & 0.9790 & 0.9915 & & 0.9790 & 0.9740 & 0.9265 & 0.9915 & 0.9905 & 0.9545 \\
\hline $10 \%$ & 1.05 & 0.0310 & 0.0415 & & 0.0985 & 0.0990 & 0.0980 & 0.0885 & 0.0900 & 0.0905 \\
& 1.10 & 0.0810 & 0.1895 & & 0.1375 & 0.1170 & 0.1150 & 0.1950 & 0.1590 & 0.1500 \\
& 1.15 & 0.2365 & 0.4270 & & 0.2190 & 0.1685 & 0.1260 & 0.3655 & 0.2675 & 0.2080 \\
& 1.20 & 0.4755 & 0.6810 & & 0.4605 & 0.3485 & 0.2275 & 0.6265 & 0.4870 & 0.3505 \\
& 1.25 & 0.7565 & 0.8720 & & 0.7495 & 0.6300 & 0.4135 & 0.8670 & 0.7520 & 0.5430 \\
& 1.30 & 0.8965 & 0.9590 & & 0.8950 & 0.8450 & 0.6570 & 0.9575 & 0.9105 & 0.7610 \\
& 1.35 & 0.9660 & 0.9860 & & 0.9660 & 0.9580 & 0.8415 & 0.9860 & 0.9775 & 0.9105 \\
& 1.40 & 0.9910 & 0.9985 & & 0.9910 & 0.9895 & 0.9605 & 0.9985 & 0.9980 & 0.9830 \\
\hline
\end{tabular}


Tabela 5.8: Taxas de rejeição para os testes de Wald e Distância $\left(H: \beta_{1}^{2}=\beta_{2}^{2}=\right.$ $\left.1, K: 1 \geq \beta_{1}^{2} \geq \beta_{2}^{2}\right)$

\begin{tabular}{|c|c|c|c|c|}
\hline$n$ & Nível & $\beta_{2}$ & Wald & Distância \\
\hline \multirow[t]{16}{*}{100} & \multirow[t]{8}{*}{$5 \%$} & 1.00 & 0.0575 & 0.0645 \\
\hline & & 0.99 & 0.0615 & 0.1015 \\
\hline & & 0.98 & 0.0905 & 0.1480 \\
\hline & & 0.97 & 0.1220 & 0.1945 \\
\hline & & 0.96 & 0.1775 & 0.2780 \\
\hline & & 0.95 & 0.2305 & 0.3365 \\
\hline & & 0.94 & 0.3210 & 0.4575 \\
\hline & & 0.93 & 0.4045 & 0.5455 \\
\hline & \multirow[t]{8}{*}{$10 \%$} & 1.00 & 0.0990 & 0.1140 \\
\hline & & 0.99 & 0.1170 & 0.1725 \\
\hline & & 0.98 & 0.1410 & 0.2205 \\
\hline & & 0.97 & 0.1910 & 0.2990 \\
\hline & & 0.96 & 0.2665 & 0.3940 \\
\hline & & 0.95 & 0.3370 & 0.4720 \\
\hline & & 0.94 & 0.4035 & 0.5660 \\
\hline & & 0.93 & 0.5055 & 0.6485 \\
\hline \multirow[t]{16}{*}{1000} & \multirow[t]{8}{*}{$5 \%$} & 1.00 & 0.0525 & 0.0530 \\
\hline & & 0.99 & 0.1045 & 0.1780 \\
\hline & & 0.98 & 0.3090 & 0.4435 \\
\hline & & 0.97 & 0.5705 & 0.7270 \\
\hline & & 0.96 & 0.8420 & 0.9195 \\
\hline & & 0.95 & 0.9675 & 0.9850 \\
\hline & & 0.94 & 0.9950 & 0.9975 \\
\hline & & 0.93 & 0.9995 & 1.0000 \\
\hline & \multirow[t]{8}{*}{$10 \%$} & 1.00 & 0.1010 & 0.1130 \\
\hline & & 0.99 & 0.1880 & 0.2995 \\
\hline & & 0.98 & 0.4085 & 0.5735 \\
\hline & & 0.97 & 0.7050 & 0.8215 \\
\hline & & 0.96 & 0.8850 & 0.9490 \\
\hline & & 0.95 & 0.9775 & 0.9935 \\
\hline & & 0.94 & 0.9985 & 1.0000 \\
\hline & & 0.93 & 0.9995 & 1.0000 \\
\hline
\end{tabular}


Tabela 5.9: Volume Testicular de Adolescentes (em ml)

\begin{tabular}{|c|c|c|c|}
\hline \multirow{2}{*}{$\begin{array}{l}\text { Número do } \\
\text { Adolescente }\end{array}$} & \multicolumn{3}{|c|}{ Método de Medição } \\
\hline & Ultrasom (padrão) & Gráfico (1) & "Ring Orchidometer" (2) \\
\hline 1 & 4.8 & 5.0 & 8.0 \\
\hline 2 & 5.1 & 5.0 & 8.0 \\
\hline 3 & 10.3 & 7.5 & 12.0 \\
\hline 4 & 2.4 & 3.5 & 4.5 \\
\hline 5 & 4.4 & 5.0 & 6.0 \\
\hline 6 & 5.7 & 5.0 & 8.0 \\
\hline 7 & 4.3 & 3.5 & 6.0 \\
\hline 8 & 5.9 & 5.0 & 8.0 \\
\hline 9 & 8.0 & 5.0 & 10.0 \\
\hline 10 & 9.5 & 10.0 & 11.0 \\
\hline 11 & 16.3 & 10.0 & 15.0 \\
\hline 12 & 7.0 & 5.0 & 9.0 \\
\hline 13 & 16.4 & 15.0 & 20.0 \\
\hline 14 & 2.3 & 2.0 & 3.0 \\
\hline 15 & 15.3 & 15.0 & 20.0 \\
\hline 16 & 22.8 & 12.5 & 20.0 \\
\hline 17 & 9.7 & 5.0 & 11.0 \\
\hline 18 & 3.7 & 3.5 & 6.0 \\
\hline 19 & 12.3 & 10.0 & 12.0 \\
\hline 20 & 9.5 & 7.5 & 10.0 \\
\hline 21 & 9.2 & 10.0 & 15.0 \\
\hline 22 & 21.7 & 10.0 & 25.0 \\
\hline 23 & 3.6 & 2.0 & 6.0 \\
\hline 24 & 3.3 & 2.0 & 4.0 \\
\hline 25 & 9.6 & 7.5 & 10.0 \\
\hline 26 & 8.0 & 10.0 & 12.0 \\
\hline 27 & 6.5 & 5.0 & 10.0 \\
\hline 28 & 6.1 & 5.0 & 7.0 \\
\hline 29 & 5.5 & 5.0 & 10.0 \\
\hline 30 & 17.4 & 15.0 & 22.5 \\
\hline 31 & 20.7 & 20.0 & 25.0 \\
\hline 32 & 13.8 & 10.0 & 15.0 \\
\hline 33 & 9.9 & 10.0 & 15.0 \\
\hline 34 & 6.8 & 7.5 & 10.0 \\
\hline 35 & 10.6 & 10.0 & 15.0 \\
\hline 36 & 9.5 & 10.0 & 11.0 \\
\hline 37 & 20.7 & 20.0 & 22.5 \\
\hline 38 & 8.1 & 5.0 & 12.0 \\
\hline 39 & 8.4 & 7.5 & 11.0 \\
\hline 40 & 2.8 & 3.5 & 6.0 \\
\hline
\end{tabular}


Tabela 5.10: Estimativas dos Parâmetros e das Variâncias Assintóticas (para parâmetros de interesse)

\begin{tabular}{ccc}
\hline Parâmetro & Estimativa & Estimativa da Variância \\
\hline$\alpha_{1}$ & 0.0123 & 0.0110 \\
$\alpha_{2}$ & 0.3909 & 0.0105 \\
$\beta_{1}$ & 0.9448 & 0.0026 \\
$\beta_{2}$ & 0.8976 & 0.0024 \\
$\mu_{x}$ & 2.0352 & \\
$\sigma_{x}^{2}$ & 0.1591 & \\
$\sigma^{2}$ & 0.0085 & \\
\hline
\end{tabular}




\section{Conclusões, Contribuições e Propostas para Futuras Pesquisas}

Acreditamos que as seguintes contribuições foram dadas por esta tese:

- No capítulo 2, uma apresentação rigorosa da teoria referente ao teste de distância. Alguns resultados apresentados não são novos, como a distribuição nula assintótica da estatística de distância, encontrada em Kodde \& Palm (1986). No entanto, na nossa opinião, o esboço de demonstração apresentado neste último trabalho não é suficiente para uma compreensão satisfatória do processo de formulação do teste. O resultado no Teorema 2.2.4 já havia sido citado em Wolak (1991), mas sem demonstração;

- No capítulo 3, a obtenção de uma expressão fechada para a estatística de distância, quando temos o modelo de regressão linear simples passando pela origem, em $k \geq 2$ populações independentes;

- No capítulo 5, a modificação sugerida para a estatística de distância;

- Em todos os capítulos, as comparações entre as funções poder de diversos testes já estabelecidos e a do teste de distância;

- A literatura para testes de hipóteses com restrição em modelos com erros nas variáveis praticamente inexistia, de modo que este modesto estudo pode servir de referencial para pesquisas futuras.

Chegamos às seguintes conclusões:

- É extremamente importante levar em consideração, conjuntamente, as suposições de erros nas variáveis/hipóteses restritas, quando da formulação de testes para hipóteses do tipo das que foram discutidas na tese. Isto fica claro quando 
verificamos, nos estudos por simulação, que alguns testes existentes na literatura não são adequados para a análise do problema. O teste de distância revelou-se superior, exatamente por ser construído de acordo com a natureza dos dados;

- O teste de distância é de fácil aplicação em situações práticas, quando o número de restrições nas hipóteses de interesse é menor ou igual a 3. Neste caso, devido ao ganho de poder revelado nos estudos por simulação, é vantajoso utilizá-lo em detrimento de um outro teste que seja mais simples em termos de necessidades computacionais, como, por exemplo, o teste de Wald, mas que não é adequado para o tipo de problema que tratamos aqui. Para um número maior de restrições, o cálculo das probabilidades de significância no teste de distância se torna uma tarefa onerosa, e precisamos recorrer a algoritmos especializados. A escolha entre um e outro teste já não se torna tão clara;

- O teste de distância apresenta deficiências características de todos os testes que são do "tipo Wald" e uma delas é apresentada no capítulo 5: no caso do modelo de calibração comparativa, existe uma região no espaço paramétrico, uma vizinhança de pontos da hipótese nula, onde a função poder do teste apresenta um comportamento pífio;

Temos as seguintes propostas para futuras pesquisas:

- Elaborar testes para hipóteses restritas em modelos de regressão com erros nas variáveis utilizando outros procedimentos gerais. Temos, em princípio, duas sugestões. A primeira é desenvolver o teste da razão de verossimilhança. Neste caso, temos também que resolver o problema da estimação de máxima verossimilhança em espaços paramétricos restritos. A segunda, é utilizar o teste sugerido por Follmann (1996). A implementação deste teste é mais simples que a do teste de distância, notadamente quando o número de restrições nas hipóteses de interesse é maior que 3;

- Estender a modificação do teste de distância feita no capítulo 5 para hipóteses com um número de restrições maior que 1 ;

- Estender os estudos feitos na tese e, possivelmente, os citados nos itens anteriores, para uma classe maior de modelos de regressão com erros nas variáveis, como os modelos funcionais, ultraestruturais, elípticos, etc. 


\section{Apêndice A}

\section{Procedimento Computacional para o Cálculo da Estatística de Distância}

Para obtermos o valor da estatística de distância, é necessário resolver o problema quadrático

$$
\begin{array}{ll}
\text { minimizar } & (z-x)^{\prime} A(z-x) \\
\text { sujeito a } & x \geq 0,
\end{array}
$$

onde $A: p \times p$ é positiva definida e $z$ é um vetor fixo em $\mathbb{R}^{p}$. Resolvê-lo é equivalente a encontrar a solução de

$$
\begin{array}{ll}
\text { minimizar } & -z^{\prime} A x+\frac{1}{2} x^{\prime} A x \\
\text { sujeito a } & x \geq 0 .
\end{array}
$$

Temos que $x \geq 0$ é solução se e somente se existe $\mu \geq 0 \mathrm{em} \mathbb{R}^{p}$ tal que

$$
\begin{aligned}
-A z+A x & =\mu \\
\mu^{\prime} x & =0 \\
x & \geq 0 .
\end{aligned}
$$

Estas são as chamadas condições de Kuhn-Tucker (Luenberger, 1984, pg. 314). Existem vários algoritmos para a resolução do problema quadrático. Nesta tese utilizamos a sugestão apresentada no Cap. 11 de Bazaraa \& Shetty (1979). Primeiramente, mostraremos que existe uma equivalência entre o problema quadrático e um outro, 
denominado problema linear complementar (PLC). Defina então

$$
B=\left(\begin{array}{cc}
A & -I_{p} \\
I_{p} & 0
\end{array}\right),
$$

$\lambda=\left(\mu^{\prime}, x^{\prime}\right)^{\prime}, w=\left(x^{\prime}, 0\right)^{\prime}$ e $d=\left(-z^{\prime} A, 0\right)^{\prime}$. As condições de Kuhn-Tucker podem ser escritas como

$$
\begin{array}{ll}
\lambda & =B w+d, \\
\lambda^{\prime} w & =0, \\
\lambda \geq 0, & w \geq 0 .
\end{array}
$$

O PLC consiste em resolver este sistema em $\lambda$ e $w$.

Em toda a tese utilizamos, para resolver o PLC, a subrotina $\mathrm{LCP}^{1}$, incluída no procedimento IML do software SAS.

\footnotetext{
${ }^{1}$ Do Inglês: Linear Complementary Problem.
} 


\section{Apêndice B}

\section{Expressões para os Pesos na Distribuição Nula Assintótica da Estatística de Distância}

Seja $\mathbf{X}=\left(X_{1}, \ldots, X_{p}\right)^{\prime} \sim N_{p}(0, \Sigma), \Sigma>0$. Neste apêndice apresentamos expressões para $\omega(p, i, \Sigma)$, a probabilidade de que a projeção (segundo a norma induzida em $\mathbb{R}^{p}$ por $\Sigma$ ) de $\mathbf{X}$ sobre $\mathcal{O}_{p}$ tenha exatamente $i$ coordenadas positivas, $i=0, \ldots, p$. Estas probabilidades são os pesos na distribuição nula assintótica da estatística de distância, conforme o Teorema 2.2.2.

Nas expressões, $\rho_{i j}$ é o coeficiente de correlação entre $X_{i}$ e $X_{j}$. Aparecem também coeficientes de correlação parcial, que são definidos da seguinte maneira: $\operatorname{seja}\left(\mathrm{X}_{1}^{\prime}, \mathrm{X}_{2}^{\prime}\right)^{\prime}$ um vetor aleatório, onde $\mathbf{X}_{1}: k \times 1$ é formado por $k$ coordenadas de $\mathbf{X}, 1<k<p$, e $\mathbf{X}_{2}:(p-k) \times 1$ é formado pelas coordenadas restantes. Seja $\left(\Sigma_{i j}\right) i, j \in\{1,2\}$ uma partição da matriz de covariâncias do vetor $\left(\mathbf{X}_{1}^{\prime}, \mathbf{X}_{2}^{\prime}\right)^{\prime}, \Sigma_{11}: k \times k$. A distribuição condicional de $\mathrm{X}_{1}$ dado $\mathrm{X}_{2}$ é normal $k$-variada, com matriz de covariâncias

$$
\Sigma_{11.2}=\Sigma_{11}-\Sigma_{12} \Sigma_{22}^{-1} \Sigma_{21}
$$

(Mardia et al., 1979, teorema 3.2.4). Suponha, para facilitar a notação, que $\mathbf{X}_{1}$ é formado pelas $k$ primeiras coordenadas de $\mathbf{X}$. Sejam $1 \leq i, j \leq k$. O coeficiente de correlação parcial entre $X_{i}$ e $X_{j}$ quando $\mathrm{X}_{2}$ é mantido fixo, denotado por $\rho_{i j . k+1, \ldots, p}$, é a correlação entre $X_{i}$ e $X_{j}$ na distribuição condicional de $\mathrm{X}_{1}$ dado $\mathrm{X}_{2}$, ou seja

$$
\rho_{i j . k+1, \ldots, p}=\frac{\left(\Sigma_{11.2}\right)_{i j}}{\left[\left(\Sigma_{11.2}\right)_{i i}\left(\Sigma_{11.2}\right)_{j j}\right]^{1 / 2}} .
$$


Tabela B.1: Expressões para os Pesos na Distribuição Nula Assintótica da Estatística de Distância

\begin{tabular}{lll}
\hline$p$ & $i$ & $w(p, i, \Sigma)$ \\
\hline 1 & 0 & $1 / 2$ \\
& 1 & $1 / 2$ \\
\hline 2 & 0 & $(1 / 2) \pi^{-1} \arccos \left(\rho_{12}\right)$ \\
& 1 & $1 / 2$ \\
& 2 & $1 / 2-(1 / 2) \pi^{-1} \arccos \left(\rho_{12}\right)$ \\
\hline 3 & 0 & $1 / 2-\omega(3,2, \Sigma)$ \\
& 1 & $1 / 2-\omega(3,3, \Sigma)$ \\
& 2 & $(1 / 4) \pi^{-1}\left(3 \pi-\arccos \left(\rho_{12.3}\right)-\arccos \left(\rho_{13.2}\right)-\arccos \left(\rho_{23.1}\right)\right)$ \\
& 3 & $(1 / 4) \pi^{-1}\left(2 \pi-\arccos \left(\rho_{12}\right)-\arccos \left(\rho_{13}\right)-\arccos \left(\rho_{23}\right)\right)$ \\
\hline 4 & 0 & $(1 / 2)-\omega(4,4, \Sigma)-\omega(4,2, \Sigma)$ \\
& & $(1 / 8) \pi^{-1}\left(-4 \pi+\sum_{\{i>j ; i, j \neq k\}} \arccos \left(\rho_{i j . k}\right)\right)$ \\
& 1 & \\
& 2 & $(1 / 4) \pi^{-2} \sum_{\{i>j, k>l ; k, l \neq i, j\}}\left(\arccos \left(\rho_{i j}\right)\right)\left(\pi-\arccos \left(\rho_{k l . i j}\right)\right)$ \\
& & $(1 / 8) \pi^{-1}\left(8 \pi-\sum_{\{i>j ; i, j \neq k\}} \arccos \left(\rho_{i j . k}\right)\right)$ \\
\hline
\end{tabular}


As expressões são apresentadas na tabela B.1. $\omega(4,4, \Sigma)$, ausente na tabela, pode ser obtido integrando numericamente uma densidade normal multivariada (veja o lema 2.2.1).

Suponha agora que $\Sigma$ é uma matriz diagonal. Neste caso, as coordenadas de X são variáveis aleatórias independentes e, usando o lema 2.2.1, obtemos

$$
\omega(p, i, \Sigma)=\left(\begin{array}{c}
p \\
i
\end{array}\right) \frac{1}{2^{p}} .
$$




\section{Bibliografia}

Amemiya, Y. (1982). Estimators for the Errors-in-variables Model. Ph.D. thesis, Iowa State University.

Amemiya, Y. \& Fuller, W. A. (1984). Estimation for the multivariate error-invariables model with estimated error covariance matrix. Annals of Statistics, 12.

Anderson, T. W. (1984). An Introduction to Multivariate Statistical Analysis. John Wiley and Sons, New York, second edition.

Ash, R. B. (1972). Real Analysis and Probability. Academic Press, New York.

Bartle, R. G. (1976). The Elements of Real Analysis. John Wiley and Sons, New York, second edition.

Bazaraa, M. S. \& Shetty, C. M. (1979). Nonlinear Programming (Theory and Methods). John Wiley and Sons, New York.

Bohrer, R. \& Chow, W. (1978). Weights for one-sided multivariate inference. Applied Statistics, 27, 100-104.

Chan, L. K. \& Mak, T. K. (1979). On the maximum likelihood estimation of a linear structural relationship when the intercept is known. Journal of Multivariate Analysis, 9, 304-313.

Cheng, C. L. \& Van Ness, J. W. (1999). Statistical regression with measurement error. Arnold, London.

Chopkevitch, E., Nishimura, R. T., Tu, D. G. S. \& Galea-Rojas, M. (1996). Clinical measurement of testicular volume in adolescents: Comparison of the reliability of 5 methods. The Journal of Urology, 156, 2050-2053. 
Conover, W. J. (1980). Practical Nonparametric Statistics. John Wiley and Sons, second edition.

Dolby, G. R. (1976). The ultrastructural relation: a synthesis of the functional and structural relations. Biometrika, 63, 39-50.

Follmann, D. (1996). A simple multivariate test for one-sided alternatives. Journal of the American Statistical Association, 91(434), 854-861.

Franklin, J. N. (1968). Matrix Theory. Prentice Hall, Inc., Englewood Cliffs, New Jersey.

Fuller, W. A. (1987). Measurement Error Models. John Wiley and Sons, New York.

Gleser, L. J. (1981). Estimation in a multivariate "errors in variables" regression model: large sample results. Annals of Statistics, 9(1), 24-44.

Henderson, H. V. \& Searle, S. R. (1979). Vec and vech operators for matrices, with some uses in jacobians and multivariate statistics. The Canadian Journal of Statistics, 7(1), 65-81.

Holm, S. (1979). A simple sequentially rejective multiple test procedure. Scandinavian Journal of Statistics, 6, 65-70.

Jørgensen, B. (1993). The Theory of Linear Models. Chapman and Hall, New York.

Kodde, D. A. \& Palm, F. C. (1986). Wald criteria for jointly testing equality and inequality restrictions. Econometrica, 54, 1243-1248.

Kudô, A. (1963). A multivariate analogue of the one-sided test. Biometrika, 50, 403-418.

Lehmann, E. L. (1983). Theory of Point Estimation. John Wiley and Sons, New York.

Lehmann, E. L. (1986). Testing Statistical Hypotheses. John Wiley and Sons, New York, second edition.

Lima, E. L. (1970). Análise no Espaço $\mathbb{R}^{n}$. Universidade de Brasília, Brasília.

Lima, E. L. (1977). Espaços Métricos. IMPA, Rio de Janeiro. 
Lima, E. L. (1987). Curso de Análise, volume 1. IMPA, Rio de Janeiro.

Lütkepohl, H. \& Burda, M. (1997). Modified Wald tests under nonregular conditions. Journal of Econometrics, 78, 315-332.

Luenberger, D. J. (1984). Linear and Nonlinear Programming. Addison-Wesley, Reading, second edition.

Mak, T. K. (1982). Estimation in the presence of incidental parameters. The Canadian Journal of Statistics, 10(2), 121-132.

Mardia, K. V., Kent, J. T. \& Bibby, J. M. (1979). Multivariate Analysis. Academic Press, London.

Moore, S. D. \& McCabe, P. G. (1993). Introduction of the Practice of Statistics. Freeman, New York.

Nüesch, P. C. (1966). On the problem of testing location in multivariate problems for restricted alternatives. The Annals of Mathematical Statistics, 37, 113-119.

Paula, G. A. \& Bolfarine, H. (1997). Some results on the slope of the linear regression model for the analysis of pretest-posttest data. Technical Report RT-MAE 9716, IME-USP, São Paulo.

Perlman, M. D. (1969). One-sided testing problems in multivariate analysis. The Annals of Mathematical Statistics, 40, 549-567.

Rao, C. R. (1973). Linear Statistical Inference and its Applications. John Wiley and Sons, New York, second edition.

Rockafellar, T. R. (1970). Convex Analysis. Princeton University Press, Princeton, New Jersey.

SAS Institute Inc. (1996). SAS/IML ${ }^{T M}$ User's Guide. SAS Institute Inc., Cary, NC, release 6.12 edition.

Seber, G. A. F. (1977). Linear Regression Analysis. John Wiley and Sons, New York.

Shapiro, A. (1985). Asymptotic distribution of test statistics in the analysis of moment structures under inequality constraints. Biometrika, 72, 133-144. 
Shapiro, A. (1988). Towards a unified theory of inequality constrained testing in multivariate analysis. International Statistical Review, 56(1), 49-62.

Sun, H. (1988a). A fortran subroutine for computing normal orthant probabilities of dimensions up to nine. Communications in Statistics-Simulation and Computation, 17(3), 1097-1111.

Sun, H. (1988b). A general reduction method for $n$-variate normal orthant probability. Communications in Statistics-Theory and Methods, 17(11), 3913-3921.

Wald, A. (1943). Tests of statistical hypotheses concerning several parameters when the number of observations is large. Transactions of the American Mathematical Society, 54(3), 426-482.

Wilks, S. S. (1962). Mathematical Statistics. John Wiley and Sons, New York.

Williams, E. J. (1969). Regression methods in calibration problems. Bullettin of the International Statistical Institute, 43, 17-28.

Wolak, F. A. (1987). An exact test for multiple inequality and equality constraints in the linear regression model. Journal of the American Statistical Association, 82(399), 782-793.

Wolak, F. A. (1991). The local nature of hypothesis tests involving inequality constraints in nonlinear models. Econometrica, 59(4), 981-995. 